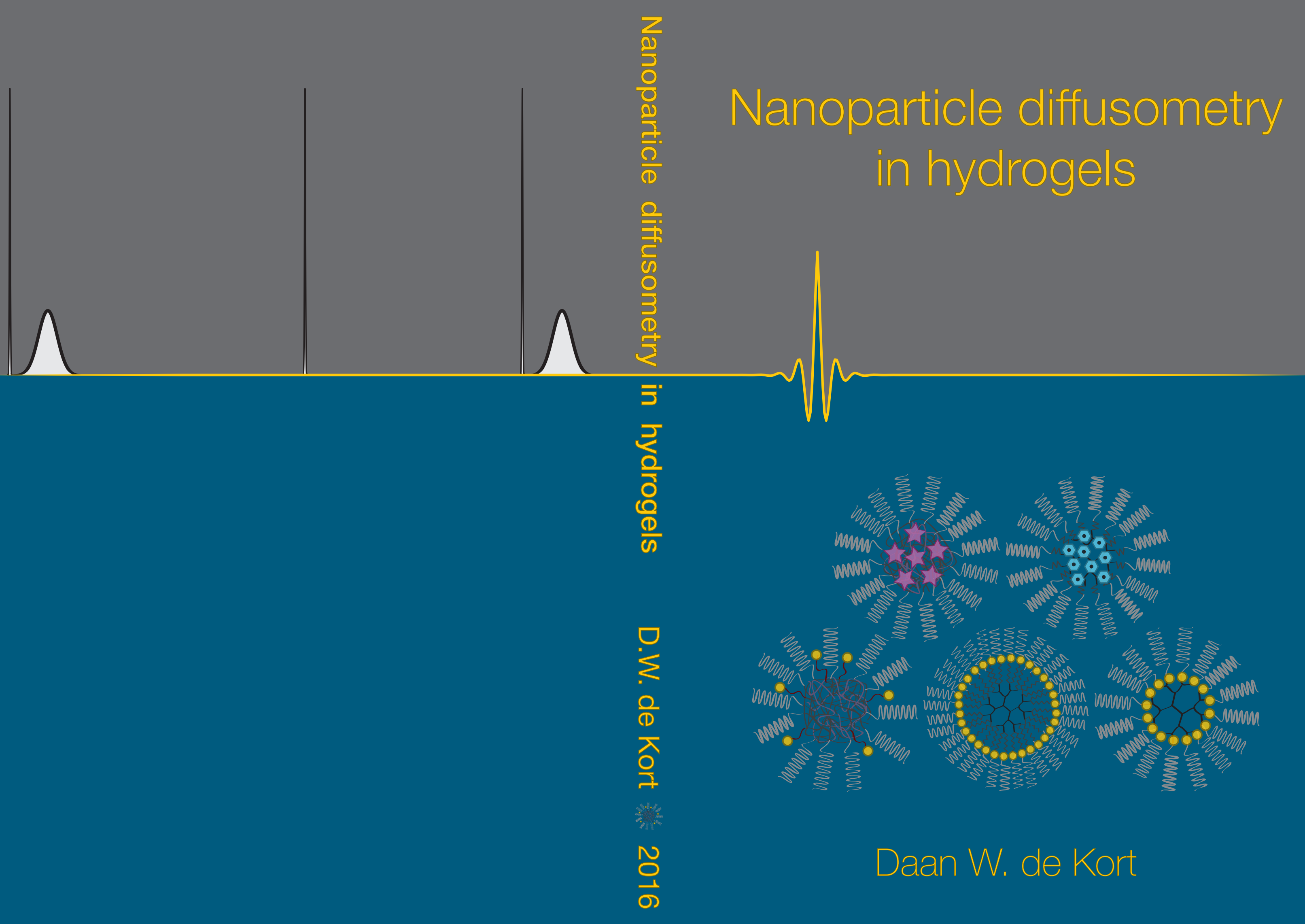


Nanoparticle diffusometry in hydrogels

Daan W. de Kort 


\section{Thesis committee}

\section{Promotor}

Prof. Dr J.P.M. van Duynhoven

Professor of Magnetic Resonance in relation to Food

Wageningen University

\section{Co-promotor}

Dr H. Van As

Associate professor, Laboratory of Biophysics

Wageningen University

\section{Other members}

Prof. Dr E. van der Linden, Wageningen University

Prof. Dr A.P.M. Kentgens, Radboud University Nijmegen, The Netherlands

Prof. Dr P.S. Belton, University of East Anglia, Norwich, United Kingdom

Prof. Dr M. Wilhelm, Karlsruhe Institute of Technology, Germany

This research was conducted under the auspices of the Graduate School VLAG (Advanced studies in Food Technology, Agrobiotechnology, Nutrition and Health Sciences). 


\title{
Nanoparticle diffusometry in hydrogels
}

\author{
Daan W. de Kort
}

\section{Thesis}

submitted in fulfilment of the requirements

for the degree of doctor

at Wageningen University

by the authority of the Rector Magnificus

Prof. Dr A.P.J. Mol,

in the presence of the

Thesis Committee appointed by the Academic Board

to be defended in public

on Tuesday 14 June 2016

at 1.30 p.m. in the Aula. 
Daan W. de Kort

Nanoparticle diffusometry in hydrogels,

178 pages.

$\mathrm{PhD}$ thesis, Wageningen University, Wageningen, NL (2016)

With references, with summary in English

ISBN 978-94-6257-745-9

DOI $10.18174 / 373875$ 


\section{Contents}

1 General introduction 1

2 Nanoparticle diffusometry for quantitative assessment of submicron 21 structure in food biopolymer networks

3 NMR nanoparticle diffusometry in hydrogels: Enhancing sensitivity and selectivity

4 Complex coacervate core micelles with spectroscopic labels for 65 diffusometric probing of biopolymer networks

5 Sub-micron structural heterogeneity in $\kappa$-carrageenan gels probed by nanoparticle diffusometry

6 Scaling behavior of dendritic nanoparticle mobility in semidilute polymer solutions

$7 \quad$ Yielding and flow of cellulose microfibril dispersions in the presence of charged polymer

8 General discussion

Acknowledgments 173

Overview of completed training activities 



\section{1}

General introduction 


\subsection{Background}

When interacting with foods, we use tools, our hands, teeth and tongue to manipulate their consistency. These interactions happen intuitively, and only rarely do we come across a completely new 'mouthfeel'. The term 'food texture' can be placed in this context: it relates to the sensory perception of the food, which is not solely determined by the physicochemical properties of the food, but also by psychological aspects (Borwankar, 1992).

From the physical point of view, food texture is therefore an ill-defined term. But the use of such terms is rooted in the complicated physical manifestations of food products, the accurate description of which requires strict definitions and nomenclature. Food products are rarely simple fluids or solids, that is, they are rarely purely viscous or elastic. The majority of food products are viscoelastic, that is, they display both fluid and solid behavior. This can be attributed to food products not just being simple solutions of small molecules but having various colloidal manifestations, such as foams, suspensions, and emulsions. These properties also lead to other characteristics typically encountered in foods, such as shear thinning (i.e., a viscosity that decreases with shear rate) and the presence of a yield stress (i.e., a finite stress that will turn the food from a solid into a fluid). This complex behavior is ultimately rooted in multi-length-scale heterogeneous network structures where both molecular and colloidal interactions are at play (Fischer \& Windhab, 2011).

In order to understand food product functionality such as elastic and flow behavior and mass transport properties, one thus first has to understand the multi-length-scale structure of the material. The aim of this thesis is to explore novel methodologies to study and characterize multilength-scale structures of food hydrogels under static and dynamic conditions. The work herein focuses on (bio)polymer hydrogels, more in particular polysaccharide gels, since polysaccharides are a crucial family of food ingredients. These gels contain percolating networks of polysaccharide strands or fibers. We will adhere to the current IUPAC definitions of 'gel' and 'hydrogel' (Alemán et al., 2007): A hydrogel is a gel in which the swelling agent is water. A gel is a non-fluid colloidal network or polymer network that is expanded throughout its whole volume by a liquid, and it has a finite yield stress.

Current microscopic and spectroscopic techniques operate at rather narrow length scales and are not well suited for microstructural characterization of semi-solid systems. Microstructural characterization of such networks by high-resolution optical or electron microscopy can be problematic because they require invasive sample preparation steps that alter the structure of the network. Exceptions are imaging methods that are combined with spectroscopic measurements, such as diffusion-weighted nuclear magnetic resonance (NMR) and magnetic resonance imaging (MRI), and fluorescence recovery after photobleaching (FRAP) confocal laser scanning microscopy (CLSM). 
The mobility of water in porous solids and biological systems is appreciated for containing a wealth of structural information (Britton, Graham, \& Packer, 2001; Brownstein \& Tarr, 1977; 1979; Mitra, Latour, Kleinberg, \& Sotak, 1995; Mitra \& Sen, 1992; Mitra, Sen, \& Schwartz, 1993; Mitra, Sen, Schwartz, \& Le Doussal, 1992; Sen, Schwartz, Mitra, \& Halperin, 1994). In particular in dense systems, water will encounter many interactions with the porous matrix due to its random Brownian motion. The extent of these interactions can be probed via spectroscopic monitoring of the translational and rotational mobility of water molecules, in particular by NMR. This approach, however, fails in soft matter such as hydrogels, in which the pores are defined by the percolating network of thin (bio)polymers. Such porous networks have a high degree of pore connectivity and often a strong degree of heterogeneity. Several NMR diffusometry studies have appeared, in which nanoparticles (or 'nanoprobes') with sizes an order of magnitude larger than water molecules were used to increase the sensitivity of the particles for the presence of the percolating network (Bernin et al., 2011; Le Feunteun \& Mariette, 2007; Lorén et al., 2009b). Although these studies demonstrated that 'nanoparticle diffusometry' is indeed quite sensitive to sub-micron structure of polymer hydrogels, and hence to changes in the structure at that length scale due to aging and degradation of the network, several bottlenecks became evident. First and foremost, in compositionally complex hydrogels, the ${ }^{1} \mathrm{H}$ NMR signal of organic nanoparticles needs to be observed against background signal from small molecules and non-rigid domains of the polymer network. Second, nanoparticles that consisted of linear polymers folded into a random coil structure could change shape depending on chemical conditions and the density of the surrounding network. This led to complex self-diffusion modes due to their ability to reptate through networks with pores smaller than the random coil size (Favre, Leonard, Laurent, \& Dellacherie, 2001).

In a next step, one would want to unravel the relationship between microstructure and dynamic conditions, such as (shear) flow encountered during processing and consumption. To this end, methods that can follow structure formation and breakdown in a non-invasive manner are required. As a non-invasive tomographic method, MRI could potentially be used to address such problems. Rheo-MRI (or: rheo-NMR) (Callaghan, 1999) has been used to study shear and pressure driven flow behavior of complex colloids such as fiber dispersions ( $\mathrm{Li}$, Seymour, Powell, \& McCarthy, 1994; Seymour, Maneval, McCarthy, McCarthy, \& Powell, 1993) and fat emulsions (Britton \& Callaghan, 2000).

This thesis will explore how nanoparticle diffusometry can be applied in food hydrogels, and what is the information content of such experiments. This introduction will first describe the key innovations that will be implemented to enhance the sensitivity and selectivity of nanoparticle diffusometry. This includes an outline of functional nanoparticle designs, complementary spectroscopic methods and their essential details, and the model system ( $\kappa$-carrageenan hydrogels) that we use to test the performance of our new methods. An extensive literature review of nanoparticle diffusometry will be provided in chapter 2 . 
A second topic of this work is the behavior of food hydrogels under dynamic (shear flow) conditions. The second part of the introduction will therefore briefly review the theoretical framework that can be used to understand the rheology of thixotropic yield-stress fluids. This framework can be used to understand the shear flow of many food gels. The experimental method that can be used to access this information, rheo-MRI, will also be described. Lastly, a model system for thixotropic and heterogeneous food hydrogels will be introduced, namely "hybrid" systems containing cellulose microfibrils (MFs) dispersed in solutions of carboxymethyl cellulose (CMC).

\subsection{Diffusional nanoparticles for structural characterization of biopolymer gels}

The famous Stokes-Einstein relation, $6 \pi \eta r D=k_{b} T$ relates the self-diffusion coefficient $D$ of a spherical particle in a homogeneous medium with its radius $r$ and the viscosity of the medium $\eta$. The size regime of the particles determines which structural and physical parameters describing the surrounding matrix can be "probed". A well-known experiment that utilizes the mobility of particles to extract physical information from viscoelastic materials is microrheology. In this experiment, micron-size colloidal particles are used to locally probe elastic properties of the network (Mason \& Weitz, 1995; Mason, Ganesan, van Zanten, Wirtz, \& Kuo, 1997; Moschakis, 2013). Particles will probe macroscopic material properties only if their size $r$ is significantly larger than mesoscopic structural features in the medium. Relative to their size, such particles diffuse in an effectively homogeneous medium. In the limit of large $r$, the macroscopic zero-shear viscosity as measured with, for instance, a lab-scale viscometer will adequately predict the diffusion coefficient of the particles.

Conversely, in the limit of small $r$, particles will be smaller than the structural features in polymer gels and solutions and hence will not probe macroscopic material properties. If the size of particles is smaller than, but on the order of, the distance between the polymer strands, the particles are still free to diffuse in the continuous phase but will be influenced by the presence of the polymers. For this reason, the self-diffusion coefficient of nanoparticles embedded in biopolymer solutions and gels of biopolymers is typically significantly lower that the self-diffusion coefficient in water (although much higher than expected based on the macroscopic zero-shear viscosity). This reduced self-diffusion coefficient contains information about the sub-micron structure of the polymer network. Several models have appeared that relate the self-diffusion coefficient of a nanoparticle with the polymer concentration (volume fraction), polymer strand thickness, nanoparticle diameter and "mesh size" (Masaro \& Zhu, 1999). A more elaborate review of such models can be found in chapter 2 .

\subsubsection{Design of functionalized nanoparticles}

A major bottleneck in the application of nanoparticle diffusometry in food hydrogels is the ability to detect them against a complex matrix of water, polymer, and small-molecular ingredients. 
Labeling the particles with spectroscopically active labels would allow their selective detection. In this thesis we restrict ourselves to the study of the diffusion of spherical, non-deformable particles. We furthermore require these particles to avoid specific interactions with the biopolymer matrix. For that purpose we propose to use a polyethylene corona and to embed the spectroscopic moieties within this outer shell.

Recent developments of synthetically highly flexible nanoparticle "scaffolds" allow for the design and synthesis of nanoparticles that fulfill these requirements. We focus on two such scaffolds, namely poly(propylene imine) (PPI) dendrimers and complex coacervate core micelles (C3Ms). Dendrimers are macromolecules with a regular and highly branched three-dimensional architecture. They are prepared through successive reaction steps ("generations"), each of which adds an additional layer of monomers on the surface, increasing their size and the number of end groups (Bosman, Janssen, \& Meijer, 1999). Seminal work by Lorén et al. has demonstrated that dendrimers can be used as sensitive diffusional nanoparticles (Lorén et al., 2009b; Lorén, Nydén, \& Hermansson, 2009a). Lorén et al. used poly(amido amine) (PAMAM) dendrimers, while in this work we will work with less costly and more stable PPI dendrimers that have amine groups at their surfaces easily accessible for further functionalization. Due to the iterative synthetic process, dendrimers are essentially monodisperse in molecular weight, and therefore also monodisperse in size (Bosman et al., 1999). Depending on the number of generations, PPI dendrimer sizes range between $1-10 \mathrm{~nm}$.

C3Ms are self-assembled organic nanoparticles formed by mixing aqueous solutions of a polyionneutral diblock copolymer and an oppositely charged polyelectrolyte (Cohen Stuart, Hofs, Voets, \& de Keizer, 2005; Voets, de Keizer, \& Cohen Stuart, 2009). The micellar core consists of a polyelectrolyte complex that is stabilized by a corona of electro-neutral segments of the diblock copolymer. These nanoparticles are formed spontaneously and reversibly. The major advantage of these micelles is that no hydrophobic entities are needed and that the overall micelle is charge neutral. The radius of the core is typically about $10 \mathrm{~nm}$ and the hydrodynamic radius of the micelles is in the order of $25 \mathrm{~nm}$, depending on the copolymer length, which is larger than dendrimers.

The major design challenge for both types of particles is the incorporation of spectroscopically active labels into the micellar cores. These labels can include organic fluorescent dyes for fluorescence-based diffusometry, ${ }^{19} \mathrm{~F}$ for background free NMR-diffusometry, or TEMPO spin labels for measurement of water diffusion coefficients in the immediate vicinity of the nanoparticles by means of Overhauser dynamic nuclear polarization (Armstrong \& Han, 2009; Franck, Pavlova, Scott, \& Han, 2013). 


\subsubsection{Experimental methodology}

NMR diffusometry. Pulsed field gradient (PFG) NMR is widely used to study structural properties of, and transport properties in foods and porous media in general (Van Duynhoven, Voda, Witek, \& Van As, 2010). PFG NMR is used to measure the ensemble average propagator or self-Van Hove function $\bar{P}(r, t)$ that describes the probability of a particle moving over $r$ during observation time $t$. For the well-known case of Brownian motion in an isotropic homogeneous medium, the propagator is a Gaussian function, $\bar{P}(\boldsymbol{r}, t)=(4 \pi D t)^{-\frac{3}{2}} e^{-\frac{r^{2}}{4 D t}}$, whose width is proportional to the diffusion coefficient $D$, a scalar property in an isotropic medium.

Experimentally, a pair of short field gradient pulses that straddles an inversion RF pulse (effectively inverting the polarity of the second gradient pulse) and that is separated by a waiting time $t$ "phase encode" the initial and final position of the nuclear spins in the sample, which leads to attenuation of the signal if there is incoherent motion (e.g. Brownian motion) or coherent motion (i.e., flow) of spin-bearing particles during time $t$. The dephasing and rephasing power of the gradient pulses is given by wavevector $\boldsymbol{q}=\gamma \delta \boldsymbol{g}$, where $\gamma$ is the gyromagnetic ratio of the nuclei $[\mathrm{rad} /(\mathrm{T} \cdot \mathrm{s})], \delta$ the width $[\mathrm{s}]$ of the gradient pulses, and $\boldsymbol{g}$, a vector, the amplitude of the gradient pulses $[\mathrm{T} / \mathrm{m}]$. In case the pulse width $\delta$ is much smaller than the observation time $t$ (narrow gradient pulse approximation), the attenuation of the NMR signal intensity as a function of vector $q$ and waiting time $t$ reads $E(\boldsymbol{q}, t)=\int \bar{P}(\boldsymbol{r}, t) e^{i \boldsymbol{q} \cdot \boldsymbol{r}} d \boldsymbol{r}$ (Callaghan, 2011). This means that there exists a Fourier relationship between the signal attenuation $E(\boldsymbol{q}, t)$ and the ensemble average propagator $\bar{P}(\boldsymbol{r}, t)$. In this picture, the contribution of (Gaussian) diffusion to the attenuation is exponential attenuation $E(\boldsymbol{q}, t)=e^{-q^{2} D t}$, while coherent flow with velocity $\boldsymbol{v}$ induces a phase modulation $E(\boldsymbol{q}, t)=e^{i \boldsymbol{q} \cdot \boldsymbol{v} t}$. PFG NMR is thus an exceptionally powerful tool to study both diffusion and flow of spin-bearing particles. It can be used, for instance, to probe the difference in diffusion coefficients between bulk and surface water in porous media (Weber, Sederman, Mantle, Mitchell, \& Gladden, 2010) or to probe the shape of the pores themselves (Callaghan, 1995; Callaghan, Coy, MacGowan, Packer, \& Zelaya, 1991; Mitra et al., 1992; Seymour \& Callaghan, 1996).

NMR relaxometry. NMR relaxometry is the study of the decay of NMR signal intensity in time. The longitudinal relaxation time $T_{1}$ is the decay constant for the recovery of the nuclear spin magnetization along the magnetic field towards its equilibrium value. The transverse relaxation time $T_{2}$ reflects decay of the NMR signal perpendicular to the magnetic field due to decoherence of the nuclear spin magnetization, which may have many causes, such as for instance (local) fluctuations in the magnetic field due to magnetic dipolar coupling. Brownstein and Tarr have long ago shown how $T_{2}$ can be used to infer geometrical information about porous media where the walls act as "sinks" that shorten the relaxation time (Brownstein \& Tarr, 1979). More recently it has been shown how the ratio between $T_{1}$ and $T_{2}$ can be used to study the strength of molecular surface interactions in porous media. The ratio between $T_{1}$ and $T_{2}$ is sensitive to such interactions 
because local magnetic field gradients close to the pore wall-liquid interface will cause dephasing of spins diffusing through these gradients ( shortening $T_{2}$ ), while not affecting $T_{1}$ (Weber, Mitchell, McGregor, \& Gladden, 2009).

Fluorescence-based diffusometric methods. There is a limited selection of experimental methods that has the sensitivity to quantitatively determine diffusion coefficients of nanoparticles in the size range used in this work $(1-25 \mathrm{~nm})$. Fluorescence recovery after photobleaching (FRAP) is a confocal laser spectroscopy method that relies on quenching the fluorescence in a micrometer-size spot in the sample using a high-power laser pulse, and monitoring the return of fluorescence in this spot as a function of time. A diffusion coefficient can then be inferred from a model describing the three-dimensional diffusion process into the bleaching spot. (Axelrod, Koppel, Schlessinger, Elson, \& Webb, 1976; Braeckmans, Peeters, Sanders, De Smedt, \& DeMeester, 2003) Fluorescence correlation spectroscopy (FCS) is a confocal microscopy method that monitors the fluctuations in the fluorescence intensity within the confocal spot. Subsequently, the intensity autocorrelation function is calculated from the time trace. Again a diffusion model is used to derive a diffusion coefficient from the autocorrelation function (Haustein \& Schwille, 2007; Koynov \& Butt, 2012; Rathgeber, Beauvisage, Chevreau, Willenbacher, \& Oelschlaeger, 2009). We will leave further discussion of the details of these fluorescence methods to chapter 2 and the successive experimental chapters. The essential point here is that FRAP relies on bleaching of particle fluorescence, while FCS and other methods require particle fluorescence to be stable. This conflict in demands on the fluorescence properties of the nanoparticles is another important reason why we choose to use C3M and dendritic particles and label them with fluorescent dyes instead of using intrinsically fluorescent nanoparticles such as gold colloid (Omari, Aneese, Grabowski, \& Mukhopadhyay, 2009) or quantum dots (Resch-Genger, Grabolle, Cavaliere-Jaricot, Nitschke, \& Nann, 2008). These intrinsically fluorescent particles cannot be bleached and therefore FRAP is precluded, whereas a clever choice of fluorescent dye would allow the use of both FRAP and FCS (and other methods as detailed in chapter 2).

Overhauser DNP-enhanced NMR spectroscopy. Rather than probing the ensemble average propagator of the diffusional nanoparticles themselves by PFG NMR diffusometry, interesting information about the water diffusion coefficient in the immediate vicinity of the nanoparticles can be obtained through Overhauser dynamic nuclear polarization-enhanced NMR spectroscopy (ODNP NMR). ODNP NMR takes advantage of the strong, time-dependent dipolar coupling between unpaired electrons and solvent nuclei to enhance the NMR signal through polarization transfer from the electron spin on a spin label (e.g., TEMPO) to nuclear spins (e.g., water ${ }^{1} \mathrm{H}$ ) in its immediate vicinity (up to $\sim 1.5 \mathrm{~nm}$ ) via cross-relaxation mechanisms (Armstrong \& Han, 2009). The efficiency of polarization transfer is strongly dependent on the dynamics between the spin bearing particles. The degree of enhancement of the NMR signal thus carries information 
about mobility and it has been shown that local water diffusion coefficients can be determined (Franck et al., 2013). As mentioned earlier, the study of water in porous (bio)polymer hydrogels is complicated by chemical exchange of protons between water and the polymer matrix, influencing diffusion and relaxation rates. As ODNP NMR measures diffusion coefficients locally, the method does not suffer from this limitation.

\subsubsection{Model system for rigid biopolymer networks: $\kappa$-carrageenan gels}

Many models exist that describe the effect of the presence of a network of polymer strands or fibers on the diffusion of nanoparticles in the continuous phase (Masaro \& Zhu, 1999). Such models, however, predict long-term, average diffusion coefficients but do not explicitly consider the effect of microstructural heterogeneity on the diffusion coefficients of solutes at shorter time scales. Lorén et al. have shown for the first time non-Gaussian diffusion of dendrimer nanoparticles in $\kappa$-carrageenan gels on the PFG NMR time scale $\left(\sim 10^{1}-10^{2} \mathrm{~ms}\right)$ (Lorén et al., $2009 b$ ). For this reason, we choose $\kappa$-carrageenan to be our main model system to explore the sensitivity of our new methods towards structural heterogeneity.

$\kappa$-Carrageenan is a linear sulfated polysaccharide with a backbone based on a repeating disaccharide unit of $D$-galactose and 3,6-anhydro- $D$-galactose, which is found in various species of red seaweeds. It is used widely in foods as a gelling agent. Gelation of $\kappa$-carrageenan occurs upon cooling a warm aqueous solution, during which the polymer coils first form helices that subsequently aggregate in a side-by-side manner (Hermansson, 1989; Viebke, Piculell, \& Nilsson, 1994). The coil-to-helix transition, which is essential for eventual gelation, is very sensitive for binding of cations such as potassium, calcium, or sodium ions, strongly influencing the microstructure and elastic strength of the gels (Hermansson, Eriksson, \& Jordansson, 1991). The microstructure of $\kappa$-carrageenan gels can therefore essentially be controlled by the choice of cations, making it ideally suited as a model system for microstructural studies.

\subsubsection{Model system for flexible polymer networks: poly(ethylene glycol) solutions}

In case the constituent polymers are not rigid but flexible, the additional complication of the coupling of the dynamics of the nanoparticles and the polymers arises, if the dynamics occur on similar time scales (i.e., when particles and polymer coils have similar sizes). Higher polymer flexibility leads to homogenous networks already at shorter time scales. Such networks can be described with well-defined parameters such as the correlation length, which provides detailed predications of the reduction of long-time nanoparticle diffusion coefficients as a function of polymer concentration. A study of nanoparticle diffusion in solutions of poly(ethylene glycol), a very flexible polymer, contrasts with the study of nanoparticle diffusion of rigid networks, because it can illustrate how nanoparticles probe macroscopic viscosity if the polymers are smaller than the particles, and a "local" viscosity much smaller than the macroviscosity if the particles are smaller than the polymers. This bridges the gap between understanding nanoparticle diffusion in 
homogeneous systems on the one hand (where the matrix dynamics are faster than the particle dynamics), and heterogeneous gels (where the matrix dynamics are slower than the particle dynamics) on the other hand.

\subsection{Velocimetry for probing local rheology of biopolymer gels}

A second aim of the thesis is to study the behavior of food hydrogels under dynamic (shear flow) conditions and to relate this behavior to microstructure. Foods are often comprised of a high concentration of small elements, such as droplets, fibers, or bubbles, dispersed in a liquid. The flow behavior of such materials is typically non-Newtonian, and the materials often have a yield stress and display phenomena such as shear thinning, thixotropy, and wall slip. In the following, first a brief review of the theory of the flow behavior of (thixotropic) yield stress fluids is provided. Next, a description will be given of rheo-MRI, a powerful method to study such flows, and a description of a "model" thixotropic yield-stress fluid: cellulose microfibrils dispersed in carboxymethyl cellulose solutions.

\subsubsection{Rheology of yield-stress fluids}

Rheology is the study of the elastic and flow properties of materials; here we will focus only on flow properties. Rheometers can be equipped with various shear geometries through which shear stress can be exerted onto the sample, leading to its deformation. Cross-sections of two possible shear geometries are shown in Figure 1.1.

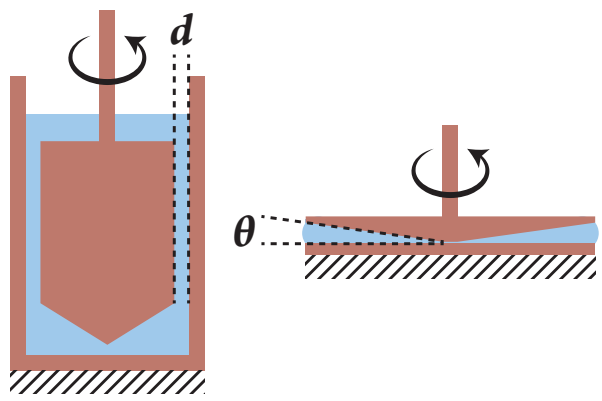

Figure 1.1: Cross sections (schematic) of concentric cylinders (CC, left), and cone-and-plate (CP, right) shear geometries. The gap width in CC geometry is $d$, the cone angle in CP geometry is $\theta$.

In these illustrations, the bottom parts of the shear geometries are fixed, while the top parts are connected to a driveshaft and eventually a motor that exerts a torque on the driveshaft. A shear stress $\sigma$ is then transferred into the sample via the rotating wall of the rotating part of the geometry. The sample is situated in the (typically) narrow gap between the rotating and fixed parts. From the resulting (local) velocity $v$ of the rotating wall, we can define the bulk shear rate $\dot{\gamma}_{b}=v / d$, where $d$ is the (local) width of the gap. The essential difference between the CC and CP geometries is, that 
while the stress field in CP geometry is (nearly) homogeneous across the gap, the stress field in CC geometry decreases with $1 / r^{2}$, where $r$ is the position in the gap, due to the effective shear surface increasing with $r^{2}$. At the same time, the length of the circular path that the sheared fluid travels under shear does not change across the gap in CP geometry, while it increases linearly with $r$ in CC geometry.

Couette and Taylor-Couette flow. Shear flow in CC and CP geometries can be approximated with the Taylor-Couette and Couette flows, respectively. Taylor-Couette flow occurs between two infinite concentric cylinders where the inner cylinder rotates, while Couette flow occurs between two infinitely large plates where one plate is stationary and the other one moves. We now consider the simple case of a Newtonian fluid undergoing Couette or Taylor-Couette flow. Newtonian fluids, such as water and many other small-molecule fluids, have a viscosity that is independent of shear stress and shear rate. The viscosity is defined as the ratio of stress and shear rate and for Newtonian fluids is thus a constant independent of the absolute values of the stress and shear rate. In Couette flow, the stress across the gap is completely homogeneous across the gap. For Newtonian fluids this means that the local shear rate $\dot{\gamma}(r)=\partial v / \partial r$ is constant and hence that the flow velocity profile $v(r)$ is perfectly linear.

In Taylor-Couette flow, the stress across the gap is proportional to the surface area over which the stress distributes: $\sigma(r)=\sigma_{i} r_{i}^{2} / r^{2}$, where $\sigma_{i}$ is the stress exerted by the rotating inner cylinder and $r_{i}$ the radius of the inner cylinder. Due to the increasing length of the circular path that the fluid has to travel around the inner cylinder, the shear rate is defined as $\dot{\gamma}(r)=r \partial(v / r) / \partial r$. Velocity profiles can be calculated, the shape of which will depend solely on the ratio between radii of the inner and outer cylinders ( $r_{i}$ and $r_{o}$, resp.). If this ratio is large, the stress drop across the gap will be significant and hence the flow velocity profile will be significantly curved (Figure 1.2). If the ratio is small, the flow velocity profile will be near linear.

In practice, for CC shear geometries, this means that the bulk shear rate $\dot{\gamma}_{b}$ is an adequate approximation of the actual shear rate across the gap only when the ratio between the radii of the inner and outer cylinder approaches unity.

Non-Newtonian flow. Many fluids display non-Newtonian flow behavior, such as shear thinning and thickening. Some materials, including many foods, might additionally have a yield stress $\sigma_{y}$. The flow behavior of a material can be classified into several categories as can be seen in Figure 1.3. The curves in Figure 1.3 show the possible relationships between the shear stress and shear rate ("flow curves") for different categories of materials. The phenomenological Herschel-Bulkley constitutive equation, $\sigma=\sigma_{y}+k \dot{\gamma}^{n}$, is widely used to describe the flow curves of yield-stress fluids. According to this model, the material behaves as a solid below $\sigma_{y}$ and as a fluid above $\sigma_{y}$. In case $n<1$, the fluid is shear thinning; in case $n>1$ it is shear thickening. Parameter $k$ is a materialspecific proportionality constant. 
Couette flow

flow between infinite parallel plates homogeneous stress across gap

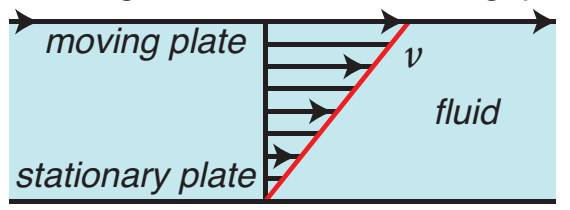

Taylor-Couette flow

flow between concentric cylinders

of infinite length

stress varies $\sim r^{-2}$ across annulus

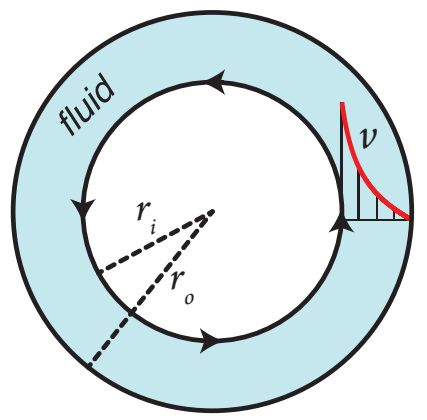

Taylor-Couette flow:

(Newtonian fluid)

$$
\text { 1. } \sigma(r)=\sigma r_{i}^{2} / r^{2}
$$

2. $\dot{\gamma}(r)=r \cdot \partial(v / r) / \partial r$

3. $\eta(r)=\sigma(r) / \dot{\gamma}(r)$
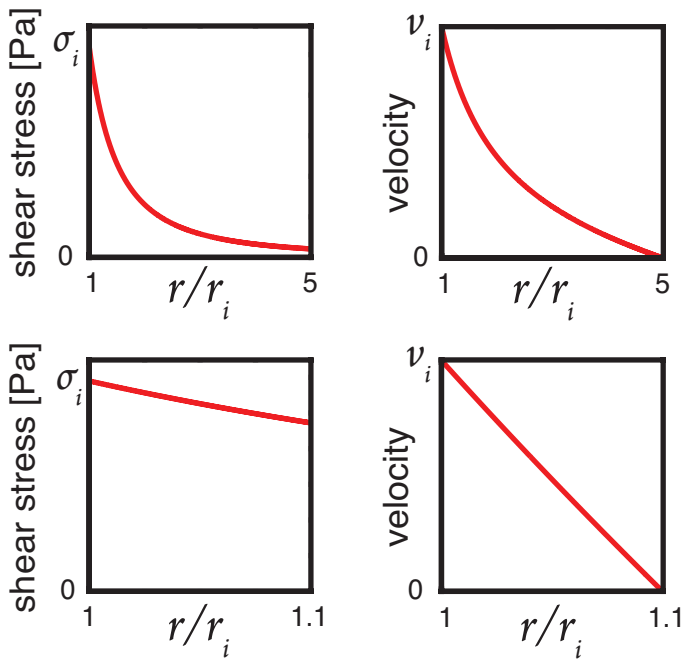

Figure 1.2: A comparison of Couette (top left) and Taylor-Couette (bottom left) flows. Two examples of Taylor-Couette stress profiles and flow velocity profiles of Newtonian fluids for two different ratios $\left(r_{o} / r_{i}=5\right.$ and 1.1) between the inner and outer cylinders are shown on the right.

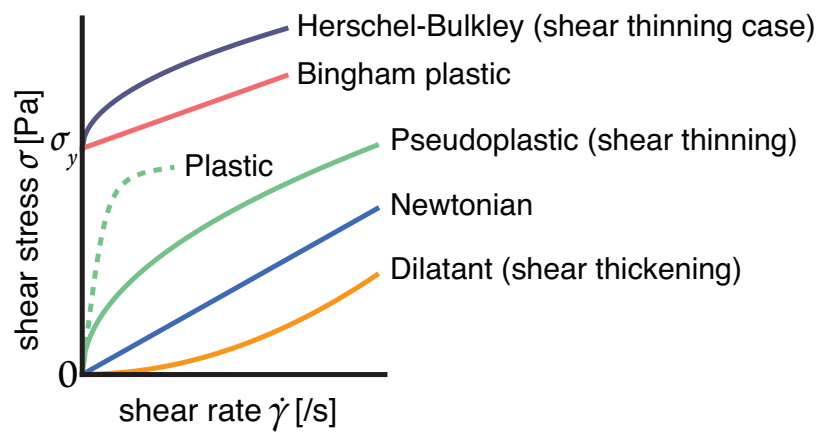

Figure 1.3: Overview of terminology for different categories of fluids and the shapes of their flow curves (shear rate against shear stress, linear scales). 


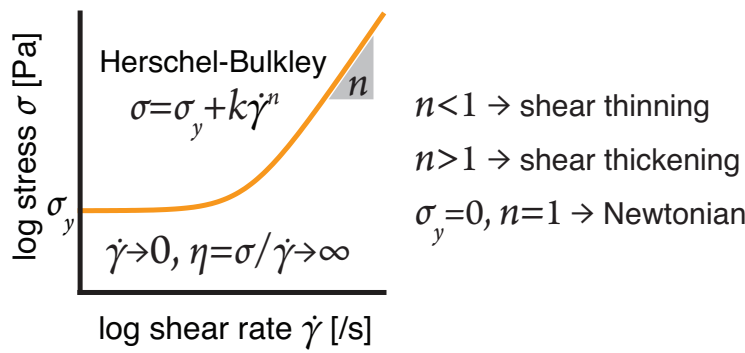

Figure 1.4: A Herschel-Bulkley curve in double-log representation; $\sigma_{y}$ is the yield stress.

The yield stress is typically determined experimentally by a logarithmic shear rate sweep, after which the Herschel-Bulkley model is fitted to obtain the yield stress, as in Figure 1.4. The Herschel-Bulkley model implies a continuous divergence of the viscosity towards infinity at low shear rates.

Thixotropy and the viscosity bifurcation. A complicating factor for the rheology of many gels and colloids is thixotropy. Thixotropy is a time-dependent shear thinning property, and after agitation, thixotropic materials will need some time to return to a solid state. Although steady-state shearcontrolled flow curves of thixotropic materials appear similar to non-thixotropic shear thinning fluids, it turns out that there is a fundamental problem with the Herschel-Bulkley model for describing the rheology of yield stress fluids: If an amount of clay suspension (a thixotropic yieldstress fluid) is deposited on a surface that is successively tilted to an angle such that the stress on the suspension is slightly higher than the apparent yield stress, the clay suspension is suddenly transported over large distances down the inclined plane ("avalanche" behavior) (Coussot, Nguyen, Huynh, \& Bonn, 2002a). The Herschel-Bulkley model, on the other hand, predicts that the viscosity at such stress values should be near infinite and the clay suspension should therefore move down the inclined plane only very slowly. The Herschel-Bulkley model thus fails to describe this behavior.

A picture similar to what is observed during inclined plane experiments emerges when the steadystate flow curve of a thixotropic material is measured in a rheometer under stress-controlled conditions (many modern rheometers are stress controlled) (Bonn et al., 2015; Coussot, 2014; Møller, Mewis, \& Bonn, 2006): After a period of shear that brings down the viscosity to a steady value, a fixed stress is applied and the system is allowed to evolve. Under such conditions, there will only be stable (permanent) flow if the imposed stress is higher than a critical value $\sigma_{c}$. The shear rate that develops when exactly the critical stress is applied, is the critical shear rate $\dot{\gamma}_{\mathrm{c}}$. At imposed stresses below $\sigma_{c}$, the viscosity will evolve towards infinity so that the flow will stop (essentially an inverse avalanche). If one then measures a flow curve under strain-controlled conditions, it becomes clear that under these conditions, there can be stable flow below the critical 
shear rate $\dot{\gamma}_{c}$, where under stress-controlled conditions the viscosity develops towards infinity. This effect is the "viscosity bifurcation" (Bonn \& Denn, 2009; Coussot, Nguyen, Huynh, \& Bonn, 2002a).

It has been debated whether at imposed stress below the critical stress (Barnes, 1997; Møller, Fall, \& Bonn, 2009) the material eventually becomes truly solid or viscous beyond the sensitivity of the rheometer. The former option, however, can be predicted analytically by assuming that thixotropic behavior is a kinetic balance between breakdown of the microscopic structure by shearing ("shear rejuvenation"), and spontaneous rebuilding of the structure in time (aging). In short, below a critical stress, aging will "win" over shear rejuvenation so that the viscosity will develop towards infinity. The implication of these models and the experimental observations is that unlike in the Herschel-Bulkley model, the steady-state flow curve of thixotropic fluids is defined only above the critical stress and critical shear rate.

Shear banding in thixotropic fluids. The critical shear rate manifests itself when shear rates lower than the critical shear rate are forced upon the material (Coussot, Raynaud, Bertrand, \& Moucheront, 2002b; Denn \& Bonn, 2010). It has been experimentally demonstrated by measuring flow velocity profiles in CP geometry (where the stress is near homogeneous) that thixotropic materials form a shear band to accommodate shear rates lower than the critical shear rate (Møller, Rodts, Michels, \& Bonn, 2008; Paredes, Shahidzadeh-Bonn, \& Bonn, 2011). At bulk shear rates higher than the critical shear rate, the local shear rate was homogeneous throughout the gap and was equal to the bulk shear rate. At bulk shear rates below the critical shear rate, a shear band was formed at the cone. The local shear rate of this band was equal to the critical shear rate. Consequently, a zero-shear band formed at the plate. This experiment was the first demonstration of shear banding in a homogeneous stress field, and furthermore lent significant credibility to the viscosity bifurcation model.

\subsubsection{Rheo-MRI velocimetry}

Clearly, flow visualizations provide crucial information that cannot be accessed by standard (mechanical) rheology, particularly in case complex phenomena such as shear banding are involved. One method to study the flow of complex fluids is rheo-MRI (Callaghan, 1999). As described in section 1.2.2, PFG NMR is a powerful tool to study flow, and can also be used to visualize flow fields by placing a shear geometry in a magnetic resonance imaging (MRI) facility. One of the advantages of rheo-MRI over other velocimetric methods is that it can be applied in optically intransparent materials (including many foods). Rheo-MRI has contributed greatly to the understanding of the rheology of yield-stress fluids as outlined above (Bonn et al., 2008), in particular the existence of the critical shear rate, leading to shear banding even in cone-and-plate geometry (Coussot, Raynaud, Bertrand, \& Moucheront, 2002b; Møller et al., 2008; Raynaud et 
al., 2002). Surprisingly, rheo-MRI has found relatively little attention as a technique to study the rheology of food dispersions.

\subsubsection{Model system for thixotropic biopolymer food gels: bacterial cellulose microfibrils dispersed in carboxymethyl cellulose solutions}

Ketchup and applesauce are essentially dispersions of cellulose fibers gelled in a continuous phase of pectin (Bengtsson \& Tornberg, 2011). Recently, dispersions of highly sheared cellulose fibers have been introduced as food structurants. In such dispersions, cellulose appears in its microfibrillar form due to the application of high shear that tears apart the fibers (ChingaCarrasco, 2011). Plant cellulose microfibrils have a higher aspect ratio than native plant cellulose fibers, leading to a lower percolation threshold (Lavoine, Desloges, Dufresne, \& Bras, 2012). This property makes microfibrils particularly attractive as structure modifiers in foods.

The flow behavior of cellulose microfibril dispersions, however, is still poorly understood. Recent work revealed that cellulose microfibril dispersions can only flow in a stable manner in the presence of a charged and flexible polymer and then behave as a heterogeneous yield-stress fluid (Kuijk et al., 2013; Veen, Kuijk, Versluis, Husken, \& Velikov, 2014; Veen, Versluis, Kuijk, \& Velikov, 2015). In this thesis, dispersions of relatively well-defined bacterial cellulose and carboxymethyl cellulose have been chosen as a model system for thixotropic food gels. We will use nanoparticle diffusometry to study their microstructure. The microstructural picture will then be related to the flow properties as viewed by rheo-MRI velocimetry.

\subsection{Scope and outline}

The following chapters are organized as follows. In chapter 2 an in-depth review of nanoparticle diffusometry is provided, with an emphasis on diffusion models and applications. In this chapter, we distinguish between (1) nanoparticle diffusion in (heterogeneous) polymer gels and (2) nanoparticle diffusion in solutions of (semi)flexible polymers. We adhere to this categorization throughout the rest of the thesis.

In chapters 3 and 4 we first describe the design and manufacturing of tailor-made nanoparticles that are functionalized with spectroscopic labels, and the implementation of pulsed-field gradient (PFG) NMR and optical spectroscopy toolboxes for nanoparticle diffusometry. We then use these toolboxes to measure nanoparticle self-diffusion in heterogeneous $\kappa$-carrageenan (a polysaccharide) gels. These experiments reveal bimodal nanoparticle self-diffusion (i.e., there are two nanoparticle fractions with different diffusion coefficients) as previously observed in these gels (Lorén et al., 2009b). The results suggest that the sub-micron structure of these gels is heterogeneous with a wide distribution of pore sizes at the sub-micron scale, leading to "sieving" of nanoparticles resulting in the observation of bimodal self-diffusion.

This hypothesis is further explored in chapter 5, where besides PFG NMR and optical spectroscopy, Overhauser dynamic nuclear polarization (ODNP)-enhanced NMR spectroscopy is 
employed. This method can determine the local viscosity of water surrounding the two fractions of particles. It turns out that the particle fraction with the lower apparent diffusion coefficient is in fact trapped in small, nanoscopic interstitials within the gel. The ODNP NMR experiments show that the viscosity of water surrounding the trapped particles is significantly lower than the viscosity within the larger interstitials.

Chapter 6 describes a study of nanoparticle diffusion in solutions of poly(ethylene glycol), a flexible polymer with well defined compositions and chain lengths. We use scaling laws to understand the relation between macroviscosity and "microviscosity" as apparent from the nanoparticle diffusivity. It turns out that the particles probe (near-)macroviscosity only if their size is larger than the size of the PEG polymer coils.

In chapter 7, we first employ nanoparticle diffusometry to study the sub-micron structure of dispersions of rigid cellulose microfibrils in the presence of carboxymethyl cellulose. Carboxymethyl cellulose is a charged cellulose derivative that succeeds to disperse the aggregation-prone cellulose microfibrils homogeneously at the sub-micron scale. Rheological characterization shows that the resulting dispersions are thixotropic yield-stress fluids. The flow properties of such fluids are well understood, but rheo-MRI experiments show that shear flow of apparently homogeneous cellulose dispersions does not resemble the flow behavior of typical thixotropic yield-stress fluids. We explain the differences by using a fluidity model to show that persistent micron-scale heterogeneity still dominates the flow behavior. 


\section{References}

Alemán, J. V., Chadwick, A. V., He, J., Hess, M., Horie, K., Jones, R. G., et al. (2007). Definitions of terms relating to the structure and processing of sols, gels, networks, and inorganic-organic hybrid materials (IUPAC Recommendations 2007). Pure and Applied Chemistry, 79(10), 1801-1829. http://doi.org/10.1351/pac200779101801

Armstrong, B. D., \& Han, S. (2009). Overhauser Dynamic Nuclear Polarization To Study Local Water Dynamics. Journal of the American Chemical Society, 131(13), 4641-4647. http://doi.org/10.1021/ja809259q

Axelrod, D., Koppel, D. E., Schlessinger, J., Elson, E., \& Webb, W. W. (1976). Mobility Measurement by Analysis of Fluorescence Photobleaching Recovery Kinetics. Biophysical Journal, 16(9), 1055-1069. http://doi.org/10.1016/S0006-3495(76)85755-4

Barnes, H. A. (1997). Thixotropy-a review. Journal of Non-Newtonian Fluid Mechanics, 70(1-2), 1-33. http://doi.org/10.1016/S0377-0257(97)00004-9

Bengtsson, H., \& Tornberg, E. (2011). Physicochemical characterization of fruit and vegetable fiber suspensions. I: Effect of homogenization. Journal of Texture Studies, 42(4), 268-280. http://doi.org/10.1111/j.1745-4603.2010.00275.x

Bernin, D., Goudappel, G.-J., van Ruijven, M., Altskär, A., Ström, A., Rudemo, M., et al. (2011). Microstructure of polymer hydrogels studied by pulsed field gradient NMR diffusion and TEM methods. Soft Matter, 7(12), 5711-5716. http://doi.org/10.1039/c1sm05070b

Bonn, D., \& Denn, M. M. (2009). Yield stress fluids slowly yield to analysis. Science, 324(5933), 1401-1402. http://doi.org/10.1126/science.1174217

Bonn, D., Paredes, J., Denn, M. M., Berthier, L., Divoux, T., \& Manneville, S. (2015). Yield Stress Materials in Soft Condensed Matter. arXiv.org.

Bonn, D., Rodts, S., Groenink, M., Rafaï, S., Shahidzadeh-Bonn, N., \& Coussot, P. (2008). Some Applications of Magnetic Resonance Imaging in Fluid Mechanics: Complex Flows and Complex Fluids. Annual Review of Fluid Mechanics, 40(1), 209-233. http://doi.org/10.1146/annurev.fluid.40.111406.102211

Borwankar, R. P. (1992). Food texture and rheology: a tutorial review. Journal of Food Engineering, 16(1-2), 1-16. http://doi.org/10.1016/0260-8774(92)90016-Y

Bosman, A. W., Janssen, H. M., \& Meijer, E. W. (1999). About Dendrimers: Structure, Physical Properties, and Applications. Chemical Reviews, 99(7), 1665-1688. http://doi.org/10.1021/cr970069y

Braeckmans, K., Peeters, L., Sanders, N. N., De Smedt, S. C., \& DeMeester, J. (2003). Three-Dimensional Fluorescence Recovery after Photobleaching with the Confocal Scanning Laser Microscope. Biophysical Journal, 85(4), 2240-2252. http://doi.org/10.1016/S0006-3495(03)74649-9

Britton, M. M., \& Callaghan, P. T. (2000). NMR Velocimetry Study of the Temperature Dependent Rheology of Butter, Semisoft Butter and Margarine. Journal of Texture Studies, 31(3), 245-255. http://doi.org/10.1111/j.1745-4603.2000.tb00288.x

Britton, M. M., Graham, R. G., \& Packer, K. J. (2001). Relationships between flow and NMR relaxation of fluids in porous solids. Magnetic Resonance Imaging, 19(3-4), 325-331. http://doi.org/10.1016/S0730725X(01)00244-2

Brownstein, K. R., \& Tarr, C. E. (1977). Spin-lattice relaxation in a system governed by diffusion. Journal of Magnetic Resonance, 26(1), 17-24. http://doi.org/10.1016/0022-2364(77)90230-X

Brownstein, K. R., \& Tarr, C. E. (1979). Importance of classical diffusion in NMR studies of water in biological cells. Physical Review A, 19(6), 2446-2453. http://doi.org/10.1103/PhysRevA.19.2446

Callaghan, P. T. (1995). Pulsed-Gradient Spin-Echo NMR for Planar, Cylindrical, and Spherical Pores under Conditions of Wall Relaxation. Journal of Magnetic Resonance. Series A, 113(1), 53-59. http://doi.org/10.1006/jmra.1995.1055

Callaghan, P. T. (1999). Rheo-NMR: nuclear magnetic resonance and the rheology of complex fluids. Reports on Progress in Physics, 62(4), 599-670. http://doi.org/10.1088/0034-4885/62/4/003 
Callaghan, P. T. (2011). Translational Dynamics \& Magnetic Resonance. New York: Oxford University Press.

Callaghan, P. T., Coy, A., MacGowan, D., Packer, K. J., \& Zelaya, F. O. (1991). Diffraction-like effects in NMR diffusion studies of fluids in porous solids. Nature, 351(6326), 467-469. http://doi.org/10.1038/351467a0

Chinga-Carrasco, G. (2011). Cellulose fibres, nanofibrils and microfibrils: The morphological sequence of MFC components from a plant physiology and fibre technology point of view. Nanoscale Research Letters, 6(1), 417. http://doi.org/10.1186/1556-276X-6-417

Cohen Stuart, M. A., Hofs, B., Voets, I. K., \& de Keizer, A. (2005). Assembly of polyelectrolyte-containing block copolymers in aqueous media. Current Opinion in Colloid \& Interface Science, 10(1-2), 30-36. http://doi.org/10.1016/j.cocis.2005.04.004

Coussot, P. (2014). Yield stress fluid flows: A review of experimental data. Journal of Non-Newtonian Fluid Mechanics, 211, 31-49. http://doi.org/10.1016/j.jnnfm.2014.05.006

Coussot, P., Nguyen, Q. D., Huynh, H. T., \& Bonn, D. (2002a). Viscosity bifurcation in thixotropic, yielding fluids. Journal of Rheology, 46(3), 573-17. http://doi.org/10.1122/1.1459447

Coussot, P., Raynaud, J. S., Bertrand, F., \& Moucheront, P. (2002b). Coexistence of liquid and solid phases in flowing soft-glassy materials. Physical Review. http://doi.org/10.1103/PhysRevLett.88.218301

Denn, M. M., \& Bonn, D. (2010). Issues in the flow of yield-stress liquids. Rheologica Acta, 50(4), 307-315. http://doi.org/10.1007/s00397-010-0504-3

Favre, E., Leonard, M., Laurent, A., \& Dellacherie, E. (2001). Diffusion of polyethyleneglycols in calcium alginate hydrogels. Colloids and Surfaces A, 194(1), 197-206. http://doi.org/10.1016/S09277757(01)00789-0

Fischer, P., \& Windhab, E. J. (2011). Rheology of food materials. Current Opinion in Colloid \& Interface Science, 16(1), 36-40. http://doi.org/10.1016/j.cocis.2010.07.003

Franck, J. M., Pavlova, A., Scott, J. A., \& Han, S. (2013). Quantitative cw Overhauser effect dynamic nuclear polarization for the analysis of local water dynamics. Progress in Nuclear Magnetic Resonance Spectroscopy, 74(C), 33-56. http://doi.org/10.1016/j.pnmrs.2013.06.001

Haustein, E., \& Schwille, P. (2007). Fluorescence correlation spectroscopy: novel variations of an established technique. Annual Review of Biophysics and Biomolecular Structure, 36(1), 151-169. http://doi.org/10.1146/annurev.biophys.36.040306.132612

Hermansson, A. M. (1989). Rheological and Microstructural Evidence for Transient States During Gelation of Kappa-Carrageenan in the Presence of Potassium. Carbohydrate Polymers, 10(3), 163-181. http://doi.org/10.1016/0144-8617(89)90009-X

Hermansson, A. M., Eriksson, E., \& Jordansson, E. (1991). Effects of potassium, sodium and calcium on the microstructure and rheological behaviour of kappa-carrageenan gels. Carbohydrate Polymers, 16(3), 297-320. http://doi.org/10.1016/0144-8617(91)90115-S

Koynov, K., \& Butt, H.-J. (2012). Fluorescence correlation spectroscopy in colloid and interface science. Current Opinion in Colloid \& Interface Science, 17(6), 377-387. http://doi.org/10.1016/j.cocis.2012.09.003

Kuijk, A., Koppert, R., Versluis, P., van Dalen, G., Remijn, C., Hazekamp, J., et al. (2013). Dispersions of Attractive Semiflexible Fiberlike Colloidal Particles from Bacterial Cellulose Microfibrils. Langmuir, 29(47), 14356-14360. http://doi.org/10.1021/la403397d

Lavoine, N., Desloges, I., Dufresne, A., \& Bras, J. (2012). Microfibrillated cellulose - Its barrier properties and applications in cellulosic materials: A review. Carbohydrate Polymers, 90(2), 735-764. http://doi.org/10.1016/j.carbpol.2012.05.026

Le Feunteun, S., \& Mariette, F. (2007). Impact of Casein Gel Microstructure on Self-Diffusion Coefficient of Molecular Probes Measured by 1H PFG-NMR. Journal of Agricultural and Food Chemistry, 55(26), 10764-10772. http://doi.org/10.1021/jf071982v 
Li, T. Q., Seymour, J. D., Powell, R. L., \& McCarthy, M. J. (1994). Visualization of flow patterns of cellulose fiber suspensions by NMR imaging. AIChE Journal, 40(8), 1408-1411. http://doi.org/10.1002/aic.690400812

Lorén, N., Nydén, M., \& Hermansson, A.-M. (2009a). Determination of local diffusion properties in heterogeneous biomaterials. Advances in Colloid and Interface Science, 150(1), 5-15. http://doi.org/10.1016/j.cis.2009.05.004

Lorén, N., Shtykova, L., Kidman, S., Jarvoll, P., Nydén, M., \& Hermansson, A.-M. (2009b). Dendrimer diffusion in kappa-carrageenan gel structures. Biomacromolecules, 10(2), 275-284. http://doi.org/10.1021/bm801013x

Masaro, L., \& Zhu, X. (1999). Physical models of diffusion for polymer solutions, gels and solids. Progress in Polymer Science, 24(5), 731-775. http://doi.org/10.1016/S0079-6700(99)00016-7

Mason, T. G., \& Weitz, D. A. (1995). Optical Measurements of Frequency-Dependent Linear Viscoelastic Moduli of Complex Fluids. Physical Review Letters, 74(7), 1250-1253. http://doi.org/10.1103/PhysRevLett.74.1250

Mason, T. G., Ganesan, K., van Zanten, J. H., Wirtz, D., \& Kuo, S. C. (1997). Particle Tracking Microrheology of Complex Fluids. Physical Review Letters, 79(17), 3282-3285. http://doi.org/10.1103/PhysRevLett.79.3282

Mitra, P. P., Latour, L. L., Kleinberg, R. L., \& Sotak, C. H. (1995). Pulsed-field-gradient nmr measurements of restricted diffusion and the return-to-the-origin probability. Journal of Magnetic Resonance, 114(1), 4758. http://doi.org/10.1006/jmra.1995.1104

Mitra, P., \& Sen, P. (1992). Effects of microgeometry and surface relaxation on NMR pulsed-field-gradient experiments: Simple pore geometries. Physical Review B, 45(1), 143-156. http://doi.org/10.1103/PhysRevB.45.143

Mitra, P., Sen, P., \& Schwartz, L. (1993). Short-time behavior of the diffusion coefficient as a geometrical probe of porous media. Physical Review B, 47(14), 8565-8574. http://doi.org/10.1103/PhysRevB.47.8565

Mitra, P., Sen, P., Schwartz, L., \& Le Doussal, P. (1992). Diffusion propagator as a probe of the structure of porous media. Physical Review Letters, 68(24), 3555-3558. http://doi.org/10.1103/PhysRevLett.68.3555

Moschakis, T. (2013). Microrheology and particle tracking in food gels and emulsions. Current Opinion in Colloid \& Interface Science, 18(4), 311-323. http:// doi.org/10.1016/j.cocis.2013.04.011

Møller, P. C. F., Fall, A., \& Bonn, D. (2009). Origin of apparent viscosity in yield stress fluids below yielding. EPL (Europhysics Letters), 87(3), 38004-7. http://doi.org/10.1209/0295-5075/87/38004

Møller, P. C. F., Mewis, J., \& Bonn, D. (2006). Yield stress and thixotropy: on the difficulty of measuring yield stresses in practice. Soft Matter, 2(4), 274-10. http://doi.org/10.1039/b517840a

Møller, P. C. F., Rodts, S., Michels, M. A. J., \& Bonn, D. (2008). Shear banding and yield stress in soft glassy materials. Physical Review E, 77(4), 041507. http://doi.org/10.1103/PhysRevE.77.041507

Omari, R. A., Aneese, A. M., Grabowski, C. A., \& Mukhopadhyay, A. (2009). Diffusion of Nanoparticles in Semidilute and Entangled Polymer Solutions. The Journal of Physical Chemistry B, 113(25), 8449-8452. http://doi.org/10.1021/jp9035088

Paredes, J., Shahidzadeh-Bonn, N., \& Bonn, D. (2011). Shear banding in thixotropic and normal emulsions. Journal of Physics: Condensed Matter, 23(28), 284116-9. http://doi.org/10.1088/0953$8984 / 23 / 28 / 284116$

Rathgeber, S., Beauvisage, H.-J., Chevreau, H., Willenbacher, N., \& Oelschlaeger, C. (2009). Microrheology with Fluorescence Correlation Spectroscopy. Langmuir, 25(11), 6368-6376. http://doi.org/10.1021/la804170k

Raynaud, J. S., Moucheront, P., Baudez, J. C., Bertrand, F., Guilbaud, J. P., \& Coussot, P. (2002). Direct determination by nuclear magnetic resonance of the thixotropic and yielding behavior of suspensions. 
Journal of Rheology, 46(3), 709-732. http://doi.org/10.1122/1.1463420

Resch-Genger, U., Grabolle, M., Cavaliere-Jaricot, S., Nitschke, R., \& Nann, T. (2008). Quantum dots versus organic dyes as fluorescent labels. Nature Methods, 5(9), 763-775. http://doi.org/10.1038/nmeth.1248

Sen, P., Schwartz, L., Mitra, P., \& Halperin, B. (1994). Surface relaxation and the long-time diffusion coefficient in porous media: Periodic geometries. Physical Review B, 49(1), 215-225. http://doi.org/10.1103/PhysRevB.49.215

Seymour, J. D., \& Callaghan, P. T. (1996). "Flow-diffraction" structural characterization and measurement of hydrodynamic dispersion in porous media by PGSE NMR. Journal of Magnetic Resonance, 122(1), 90 93. http://doi.org/10.1006/jmra.1996.0182

Seymour, J. D., Maneval, J. E., McCarthy, K. L., McCarthy, M. J., \& Powell, R. L. (1993). NMR velocity phase encoded measurements of fibrous suspensions. Physics of Fluids A, 5(11), 3010. http://doi.org/10.1063/1.858709

Van Duynhoven, J., Voda, A., Witek, M., \& Van As, H. (2010). Time-domain NMR applied to food products. In Annual Reports on NMR Spectroscopy (Vol. 69, pp. 145-197). http://doi.org/10.1016/S00664103(10)69003-5

Veen, S. J., Kuijk, A., Versluis, P., Husken, H., \& Velikov, K. P. (2014). Phase transitions in cellulose microfibril dispersions by high-energy mechanical deagglomeration. Langmuir, 30(44), 13362-13368. http://doi.org/10.1021/la502790n

Veen, S. J., Versluis, P., Kuijk, A., \& Velikov, K. P. (2015). Microstructure and rheology of microfibrilpolymer networks. Soft Matter, 1-6. http://doi.org/10.1039/C5SM02086G

Viebke, C., Piculell, L., \& Nilsson, S. (1994). On the Mechanism of Gelation of Helix-Forming Biopolymers. Macromolecules, 27(15), 4160-4166. http://doi.org/10.1021/ma00093a017

Voets, I. K., de Keizer, A., \& Cohen Stuart, M. A. (2009). Complex coacervate core micelles. Advances in Colloid and Interface Science, 147-148, 300-318. http://doi.org/10.1016/j.cis.2008.09.012

Weber, D., Mitchell, J., McGregor, J., \& Gladden, L. F. (2009). Comparing Strengths of Surface Interactions for Reactants and Solvents in Porous Catalysts Using Two-Dimensional NMR Relaxation Correlations. The Journal of Physical Chemistry C, 113(16), 6610-6615. http://doi.org/10.1021/jp811246j

Weber, D., Sederman, A. J., Mantle, M. D., Mitchell, J., \& Gladden, L. F. (2010). Surface diffusion in porous catalysts. Physical Chemistry Chemical Physics, 12(11), 2619-6. http://doi.org/10.1039/b921210h 



\title{
Nanoparticle diffusometry for quantitative assessment of submicron structure in food biopolymer networks
}

\begin{abstract}
At the submicron scale, food biopolymer networks can be visualized by a wide array of microscopic techniques, but these methods are mostly invasive and require careful image analysis in order to quantify network features. 'Nanoparticle diffusometry' provides a non-invasive alternative to infer quantitative submicron structural information about biopolymer networks. In this approach, spectroscopy-based methods are used to monitor hindered diffusion of nanoparticles due to network obstructions. Both rigid-spherical and flexible nanoparticles can be used and models are available to derive structural network parameters. A range of applications to polysaccharide and protein sols and gels has been described. The approach offers opportunities to assess (sub-)micron scale network heterogeneity and changes in sub-micron structure under dynamical conditions such as shear or ageing.
\end{abstract}

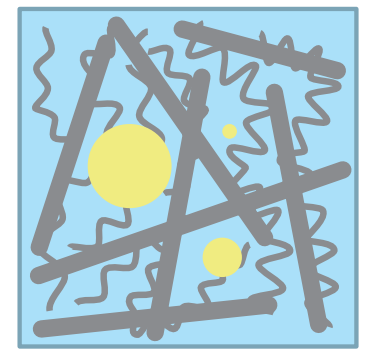

Daan W. de Kort, John P.M. van Duynhoven, Henk Van As, and François Mariette, Trends in Food Science \& Technology 2015, 42 (1), pp 13-26. DOI: 10.1016/j.tifs.2014.11.003 


\subsection{Introduction}

In foods, the structural characteristics of micro- and mesoporous biopolymer networks underlie critical macroscopic product functionalities, such as taste, mouthfeel, controlled release and delivery characteristics, and water retention capacity and barrier properties. It is important to understand and control biopolymer network structures when the formulation of a food has to be redesigned. It is therefore necessary to have information about the structural, dynamical and ageing characteristics of the biopolymer network. A direct visualization of biopolymer networks can be provided by electron microscopy techniques, but these methods suffer from several shortcomings. Sample preparation methods are invasive and likely to influence network properties. Moreover, careful image analysis is necessary in order to quantify structural descriptors and dynamic events in time cannot be studied on the same sample. Imaging techniques that provide nanometer resolution generally cover only a small field of view, which compromises representative assessment of heterogeneous systems.

Over the last few years, we have witnessed the advent of 'nanoparticle diffusometry', which uses fluorescence and optical microscopy as well as nuclear magnetic resonance (NMR)-based techniques to monitor the diffusion of nanoparticles in biopolymer networks. Hindered diffusive behavior of nanoparticles can be used to quantitatively infer submicron structural descriptors, such as mesh size or polymer strand thickness, by using models that describe nanoparticle self-diffusion in obstructing networks. Nanoparticles are used because they conveniently match mesh sizes in biopolymer gels. Self-diffusional behavior of small organic or water molecules is generally not significantly affected by the very open structures of biopolymer networks. (Gottwald, Creamer, Hubbard, \& Callaghan, 2005; Mariette, Topgaard, Jönsson, \& Söderman, 2002) Besides this, these small molecules often engage in specific physico-chemical interactions with the network, which complicates interpretation of experimental diffusometric data.

Nanoparticle diffusometry should be distinguished from direct assessment of restriction casu quo barrier functionality of a biopolymer material towards specific (bio)macromolecules. Using this approach, barrier properties of foods and other natural substances such as fish meat (CarvajalRondanelli \& Lanier, 2010), mucus and mucin (Lafitte, Söderman, Thuresson, \& Davies, 2007a; Lafitte, Thuresson, Jarwoll, \& Nydén, 2007b; Olmsted et al., 2001; B. S. Schuster, Suk, Woodworth, \& Hanes, 2013) have been described. The approach works very well in the design of products that are effective barriers against those specific (bio)molecules, where measurement of diffusion coefficients is a direct measure of barrier functionality, as opposed to microscopic images, which only provide an indirect assessment. These studies, however, do not aim at quantitatively deriving the underlying structural network descriptors through physical models of diffusion.

Also different from nanoparticle diffusometry is microrheology, where micron-size colloidal particles are used to locally probe elastic properties of the network. Micron-size particles locally probe macroscopic material properties because their size is much larger than mesoscopic structural 
features. Therefore, the particles diffuse in an effectively homogeneous medium. In microrheology, the time-dependent mean square particle displacement $\left\langle r^{2}(t)\right\rangle$ due to diffusion is recorded to derive the frequency-dependent complex visco-elastic modulus ( $G$, $G$ ') via the generalized Stokes-Einstein equation (Mason \& Weitz, 1995). Microrheology applications in food gels and emulsions mostly rely on particle tracking and diffusing wave spectroscopy. (Moschakis, 2013)

Nanoparticle diffusometry has already been used to characterize the microstructures of polysaccharide and protein networks. Examples include $\kappa$-carrageenan, galactomannan, alginate, whey and casein systems. For these systems, parameters such as polymer strand radii, mesh sizes and protein aggregate voluminosities were derived (for references: Table 2.3).

Here, we will review a range of nanoparticle diffusometry applications in food science. We will also review relevant models, instrumental methods and physico-chemical properties of various nanoparticles. We will extract principles and examples from literature directly related to foods. Yet unexploited opportunities for foods will be illustrated by recent examples from adjacent fields, which also rely on structural characterization of (bio)polymer networks. We will finalize with perspectives and trends in nanoparticle diffusometry.

\subsection{Models for nanoparticle diffusion in biopolymer networks}

As opposed to the use of diffusometry to assess barrier functionality towards specific (bio)molecules, quantitative nanoparticle diffusometry is a model-based approach to material characterization, aimed at obtaining quantitative network parameters.

Models for particle diffusion in polymer sols and gels build on established relationships describing Brownian motion of particles diffusing in a simple liquid, the diffusion coefficient follows from the Einstein-Smoluchowski relation $D f=k_{b} T$, where $D$ is the self-diffusion coefficient $\left(\mathrm{m}^{2} \mathrm{~s}^{-1}\right), f$ is the drag coefficient $\left(\mathrm{kg} \mathrm{s}^{-1}\right), k_{b}$ is the Boltzmann constant and $T$ is temperature $(\mathrm{K})$. For spherical particles in a simple liquid, the magnitude of the drag coefficient $f$ is given by Stokes' law $f=6 \pi \eta r$, where $\eta$ is the dynamic viscosity (Pa s) and $r$ is the hydrodynamic particle radius ( $\mathrm{m}$ ). Combining these equations gives the Stokes-Einstein relation $6 \pi \eta r D=k_{b} T$ for the diffusion coefficient of spherical particles in simple liquids. In this case the nanoparticle displacement probability distribution (also van Hove self-correlation function or ensemble-average propagator) is Gaussian at all time scales and the mean-square particle displacement increases linearly with time ("Fickian" diffusion) (Table 2.1, case A).

For simple liquids the drag coefficient is determined by hydrodynamic interactions of the particle with solvent and solutes. For the more complex case of self-diffusion in sols of flexible and semiflexible polymers (Table 2.1, case B), the van Hove self-correlation function is Gaussian if the movement of the polymer chains is fast with respect to particle mobility. A non-Gaussian van Hove self-correlation function has only been observed in solutions of semiflexible F-actin filaments at the millisecond timescale (Valentine et al., 2001), suggesting that observation of a non- 
Gaussian van Hove self-correlation function is related to both the observation window and the time scale of polymer dynamics. In flexible and semiflexible polymer solutions, diffusion is Fickian only if the particles are much smaller than the structural length scales in the network, but not if the nanoparticle size is comparable to or larger than the structural length scale of the network. (Brochard Wyart \& de Gennes, 2000) Although progress has been made in the conceptual description of time-dependent nanoparticle diffusion in flexible polymer networks (Cai, Panyukov, \& Rubinstein, 2011), these effects relate to (passive) microrheology and therefore fall outside the scope of this review. Here, we will focus on particle diffusion in rigid/fibrous networks (e.g. gels) where the ensemble-average propagator can be non-Gaussian and time-dependent, depending on network heterogeneity (Table 2.1, case C). In these systems, the van Hove selfcorrelation function can be non-Gaussian due to confinement of the nanoparticles within the micro- or mesoporous network, or due to heterogeneity (i.e. a large distribution of pore sizes) (Valentine et al., 2001). If the nanoparticles are not confined and the pore size distribution is finite (i.e. the material is not "fractal" at all length scales) the propagator will always be Gaussian at long time scales (Netz \& Dorfmüller, 1995; B. Wang, Kuo, Bae, \& Granick, 2012). In this case, the observed diffusion coefficient will be lower than in pure solvent and from this decrease, structural information about the network can be derived through physical models of diffusion in gels.

Two methodological approaches for probing biopolymer gels can be distinguished based on the characteristics of diffusional nanoparticles used. Nanoparticles can be either flexible and deformable when constrained to small volumes, or rigid and spherical. Models for both cases will be discussed in the following. For both types of nanoparticles, physico-chemical interactions between network and nanoparticles are not accounted for in diffusion models; In case of attractive interactions between particles and network, diffusion coefficients have been shown to be lower than predicted from physical models of diffusion (E. Schuster, Hermansson, Öhgren, Rudemo, \& Lorén, 2014). Hence, for practical applications, nanoparticles need to be selected to be "nonsticky" with regard to the network. 


\section{A. Simple, Viscous}

Liquids

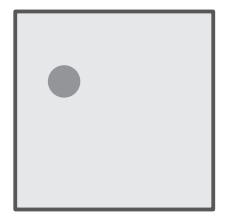

B. Sol(ution)s of Flexible and Semiflexible Polymers

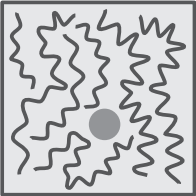

C. Rigid / Fibrous Networks (Gels)

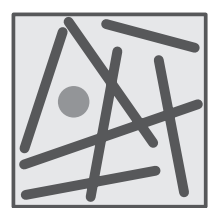

\begin{tabular}{|c|c|c|c|}
\hline $\begin{array}{r}\text { van Hove self- } \\
\text { correlation } \\
\text { function }\end{array}$ & Gaussian & $\begin{array}{l}\text { Non-Gaussian behavior has only been observed in } \\
\text { semiflexible polymer solutions for nanoparticles with a } \\
\text { size comparable to the structural length scale. (Valentine } \\
\text { et al., 2001) }\end{array}$ & $\begin{array}{l}\text { (1) Non-Gaussian at the time scale at which the nanoparticles' } \\
\text { diffusion path length is close to the structural length scale } \\
\text { (Valentine et al., 2001) } \\
\text { (2) Gaussian at long time scales (central limit theorem), if } \\
\text { nanoparticles are not permanently constrained and the system } \\
\text { is not completely fractal (Lorén, Nydén, \& Hermansson, } \\
\text { 2009a; Netz \& Dorfmüller, 1995; B. Wang et al., 2012) }\end{array}$ \\
\hline $\begin{array}{r}\text { information } \\
\text { content }\end{array}$ & Liquid homogeneity & $\begin{array}{l}\text { Network homogeneity in case a Gaussian distribution is } \\
\text { observed }\end{array}$ & $\begin{array}{l}\text { (1) Network heterogeneity } \\
\text { (2) Tortuosity }\end{array}$ \\
\hline $\begin{array}{r}\text { time-dependency } \\
\text { of mean-square } \\
\text { displacement }\end{array}$ & Linear (Fickian) & $\begin{array}{l}\text { (1) Fickian in case particles are smaller than the structural } \\
\text { length scale (Cai et al., 2011) } \\
\text { (2) Non-linear (anomalous) in case nanoparticle size is } \\
\text { comparable to or larger than the structural length scale } \\
\text { (Cai et al., 2011; Wong et al., 2004) }\end{array}$ & $\begin{array}{l}\text { Anomalous at the time scale at which nanoparticles become } \\
\text { constrained (Valentine et al., 2001) }\end{array}$ \\
\hline $\begin{array}{r}\text { information } \\
\text { content }\end{array}$ & Solvent viscosity & $\begin{array}{l}\text { (1) Solvent viscosity } \\
\text { (2) Local network elasticity (microrheology) }\end{array}$ & Network heterogeneity, pore size \\
\hline
\end{tabular}

Table 2.1: Three cases for nanoparticle diffusion: (A) Simple viscous liquids, (B) Sols of flexible and semiflexible (bio)polymers, (C) Rigid/fibrous networks, such as gels. 


\subsubsection{Diffusion models for flexible nanoparticles}

Most applications of quantitative nanoparticle diffusometry use flexible polymers as diffusional probes. Flexible particles include dextran, a branched polysaccharide, and polyethylene glycol (PEG), a linear polymer. These polymers are spherical (random coils) in dilute solutions, but deform in more crowded environments. The power law $D \propto M_{w}^{\alpha}$ gives the dependency of the diffusion coefficient of polymer chains in solution on their molecular weight $M_{w}$. The magnitude of $\alpha$ depends on the characteristics of the environment through which polymers diffuse. According to the Zimm model for diffusion of randomly coiled polymer chains in dilute solution, diffusion coefficients are inversely proportional to the size of the coil: $D \propto R^{-1}$. Flory predicted that $M_{w}^{\alpha} \propto$ $R^{5 / 3}$ (fractal dimension 5/3) for swollen linear chains in dilute solution. It follows that $\alpha=-3 / 5$ in dilute solution. In crowded environments, de Gennes predicted reptation-like diffusion of polymer chains (i.e. snake-like movement), for which $\alpha$ equals -2 (Rubinstein \& Colby, 2003). In reality, $\alpha$ often falls in-between these two situations, which could correspond to an incomplete deformation of chains into, for example, an ellipsoid shape (Figure 2.1) (Favre, Leonard, Laurent, \& Dellacherie, 2001). The molecular-weight dependency of diffusion coefficients of linear polymers can, therefore, give an estimate of the mesh size of the network. In case the polymer coil size surpasses the network mesh size, $\alpha$ shifts to more negative values.

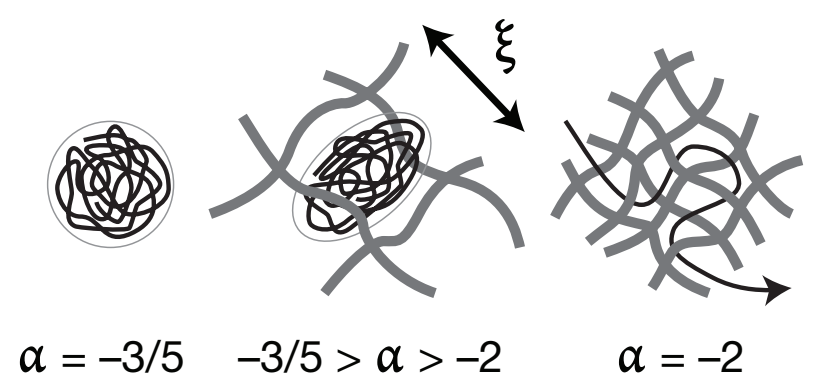

Figure 2.1: The dependency of the diffusion coefficient $D$ of a flexible nanoparticle (a polymer chain) on its molecular weight $M_{w}$ changes according to the power law $D \propto M_{w}^{\alpha}$, where the magnitude $\alpha$ depends on the degree of crowding. In one limit, nanoparticles are in dilute solution and adopt a random coil conformation $(\alpha=-3 / 5)$. Nanoparticles begin to deform when the mesh size $\xi$ is of the same order as the radius of gyration of the nanoparticle polymer coil. When $\xi$ is much smaller than the radius of gyration of the free polymer coil, unfolded chains must reptate through the crowded network $(\alpha=-2)$. Reproduced from (Favre et al., 2001), with permission from Elsevier.

We note that the term mesh size is poorly defined in most of the literature cited here. The definition depends fully on the context and the assumed structural model of the gel. Generally, the term seems to be used to describe a measure of the average distance between polymer strands or 
fibers, analogous to the correlation length in semidilute polymer solutions as defined by de Gennes (de Gennes, 1979). Sometimes, it is used to describe an average pore size, or another structuralmodel dependent length scale that can be used to describe the polymer network morphology as for example the particle-accessible volume term proposed by Babu et al. (Babu, Gimel, \& Nicolai, 2008)

\subsubsection{Diffusion models for rigid, spherical nanoparticles}

There is a large body of literature describing diffusion of rigid and spherical nanoparticles through polymer networks. Various rigid and spherical nanoparticles have been used, ranging from proteins (Silva, Peixoto, Lortal, \& Floury, 2013) to solid gold colloidal nanoparticles (Kohli \& Mukhopadhyay, 2012). A recently introduced class of spherical nanoparticles are dendrimers: macromolecules consisting of successive branched repeating units, which extend radially outward in up to $\sim 10$ consecutive layers or "generations", leading to particle diameters in the $10^{\circ} \mathrm{nm}$ range. Dendrimers are essentially monodisperse, because of controlled stepwise size increments during synthesis (Cheng, Prud'homme, \& Thomas, 2002).

In order for the nanoparticles to be non-sticky with respect to biopolymer networks, they have to be charge-neutral and free of any chemical groups that can engage in nonspecific interactions with the network. PEGylation of organic particles such as dendrimers is most often used for this purpose. To a lesser extent inert gold nanoparticles have been used (Kohli \& Mukhopadhyay, 2012). Alternative biocompatible nanoparticle coatings such as poly(vinyl acetate) (PVA) and dextran have been described (A. K. Gupta \& Gupta, 2005), but have not been applied yet to diffusional nanoparticles. The degree of rigidity of nanoparticles can be expressed by their fractal dimension $v$, defined by the power law $M_{w} \propto R^{v}$, where $R$ is nanoparticle size. Fractal dimension of a nanoparticle class can be used as a proxy for their conformation. For solid spheres, $v$ equals 3; for ideal chain random polymer coils, $v$ equals 2 . For dextrans -slightly branched polysaccharides with an expanded conformation- $v$ equals 2.3. Dendrimer fractal dimensions fall in-between 2.3 and 3 (Cheng et al., 2002).

There are many physical models that describe the diffusion of rigid and spherical nanoparticles in polymer networks as a function of structural parameters, such as nanoparticle radius, polymer volume fraction and polymer strand radius. Masaro and Zhu have reviewed different modeling approaches (Masaro \& Zhu, 1999). In another review, Amsden validated models against literature data and presented a list of models that could be reconciled with most experimental data (Amsden, 1998b). Those models that allow the derivation of structural descriptors of polymer networks have been included in Table 2.2. 


\begin{tabular}{|c|c|c|c|}
\hline $\begin{array}{l}\text { network } \\
\text { properties }\end{array}$ & model & class & references \\
\hline $\begin{array}{l}\text { Uniform random } \\
\text { suspension of fibers }\end{array}$ & $\frac{D}{D_{0}}=e^{-\frac{r_{s}+r_{f}}{r_{f}} \sqrt{\varphi}}$ & obstruction effect & (Ogston, 1958) \\
\hline $\begin{array}{l}\text { linear, rigid polymer } \\
\text { gel }\end{array}$ & $\frac{D}{D_{0}}=\left(1+\frac{2}{3} \alpha\right)^{-1}$ with $\alpha=\varphi\left(\frac{r_{s}+r_{f}}{r_{f}}\right)^{2}$ & obstruction effect & $\begin{array}{l}\text { (Tsai \& Strieder, } \\
\text { 1986) }\end{array}$ \\
\hline $\begin{array}{l}\text { closely-packed hard } \\
\text { spheres }\end{array}$ & $\frac{D}{D_{0}}=\left[1-\frac{\varphi}{0.571}\right]\left[1+\varphi^{2}\left(1.459-11.04 \varphi^{2}\right]\right.$ & obstruction effect & (Speedy, 1987) \\
\hline $\begin{array}{l}\text { linear, rigid polymer } \\
\text { gel }\end{array}$ & $\frac{D}{D_{0}}=e^{-0.84 \alpha^{1.09}}$ with $\alpha=\varphi\left(\frac{r_{s}+r_{f}}{r_{f}}\right)^{2}$ & obstruction effect & $\begin{array}{l}\text { (Johansson, Skantze, } \\
\text { \& Loefroth, 1991) }\end{array}$ \\
\hline $\begin{array}{l}\text { linear polymer gel } \\
\text { with very stiff chains, } \\
r_{s}>2 \mathrm{~nm}\end{array}$ & $\begin{array}{l}\frac{D}{D_{0}}=\frac{e^{-0.84 \alpha^{1.09}}}{1+\left(\frac{r_{s}^{2}}{k}\right)^{1 / 2}+\frac{1 r_{s}^{2}}{3 k}} \text { with } \alpha=\varphi\left(\frac{r_{s}+r_{f}}{r_{f}}\right)^{2} \text { and } k= \\
0.31 r_{f}^{2} \varphi^{-1.17}\end{array}$ & $\begin{array}{l}\text { obstruction effect } \\
\text { and hydrodynamics }\end{array}$ & $\begin{array}{l}\text { (Johnson, Berk, Jain, } \\
\text { \& Deen, 1996) }\end{array}$ \\
\hline $\begin{array}{l}\text { linear polymer gel } \\
\text { with very stiff chains, } \\
\varphi<0.40, r_{s}>2 \\
\mathrm{~nm}\end{array}$ & $\begin{array}{l}\frac{D}{D_{0}}=\left(1+\frac{2}{3} \alpha\right)^{-1} e^{-\pi \varphi^{0.174 \ln \left(51.6^{r} f / r_{s}\right)}} \text { with } \alpha= \\
\varphi\left(\frac{r_{s}+r_{f}}{r_{f}}\right)^{2}\end{array}$ & $\begin{array}{l}\text { obstruction effect } \\
\text { and hydrodynamics }\end{array}$ & $\begin{array}{l}\text { (Clague \& Phillips, } \\
\text { 1996) }\end{array}$ \\
\hline $\begin{array}{l}\text { linear, rigid polymer } \\
\text { gel }\end{array}$ & $\begin{array}{l}\frac{D}{D_{0}}=e^{-\pi\left[\frac{r_{s}+r_{f}}{k_{s} \sqrt{\varphi}+r_{f}}\right]^{2}} \text { with } k_{s} \text { an adjustable } \\
\text { parameters depending on the flexibility of the } \\
\text { polymer chains. }\end{array}$ & $\begin{array}{l}\text { obstruction effect } \\
\text { and scaling law }\end{array}$ & (Amsden, 1998a) \\
\hline
\end{tabular}

Table 2.2: Models for diffusion of spherical nanoparticles in rigid polymer gels that can be used to derive quantitative network descriptors. All models assume non-sticky nanoparticles. Meaning of the symbols: $r_{s}$ is the solute (particle) radius, $r_{f}$ is the polymer fiber radius and $\varphi$ is the polymer volume fraction. The model by Cukier et al. (Cukier, 1984) has not been listed since it has been shown to be valid mainly for small diffusants in semidilute polymer solutions (Masaro \& Zhu, 1999). The model by Ogston et al. (Ogston, 1958) has been criticized for lack of agreement to experimental data, which mainly show a Gaussian dependence of diffusivity on particle radius: $\frac{D}{D_{0}} \propto e^{-r_{s}^{2}}$ (Amsden, 1998b) Adapted with permission from (Amsden, 1998b). Copyright 1998, American Chemical Society.

Predicted diffusion coefficients should be interpreted as long-time diffusion coefficients. All models are based on the "obstruction effect"; some also include hydrodynamic arguments and generally apply only to rigid networks.

Obstruction effect models rely on the notion that the volume occupied by the rigid polymer network is inaccessible to the diffusing species, leading to an increased path length between two points in the system (Figure 2.2). 


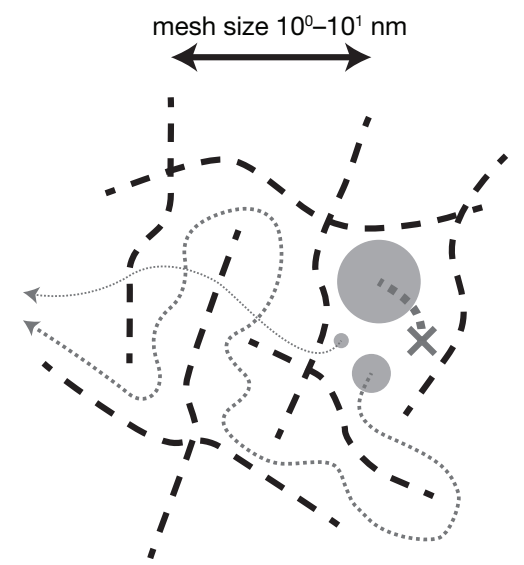

Figure 2.2: Illustration of the obstruction effect (tortuosity) in a rigid/fibrous network. Black, dashed lines indicate polymer strands. Grey, dotted lines indicate diffusion trajectories. The smallest (nano)particles can diffuse nearly freely between two points in the system, whereas intermediate-size particles have to diffuse around the barriers to reach the same end-point. The largest particles are trapped. Cf. Table 2.2 for obstruction-effect models for diffusion in rigid/fibrous polymer networks.

This leads to a decrease of the nanoparticle diffusion coefficient at long time scales, with respect to the diffusion coefficient in solvent. The factor with which the diffusion coefficient is reduced is often reported as the tortuosity (L. M. Sanders \& Hendren, 2006). In a system with the same mesh size, larger particles will experience a larger obstruction effect than smaller particles. The approach can be used only when the presumption of a motionless network holds, and is therefore particularly useful in rigid/fibrous networks (Ogston, 1958). Besides this, obstruction models obviously break down if diffusants are too large, so that hydrodynamic drag with polymers increases significantly or that diffusants are immobilized through the "sieving" action of the polymer mesh (Lauffer, 1961). The obstruction effect also lends itself to be predicted as a function of any random structure using Monte Carlo simulations (Babu et al., 2008; Kvarnström, Westergård, Lorén, \& Nydén, 2009).

It can be seen in Table 2.2 that obstruction-effect based models mainly predict polymer strand radii. The combination of polymer strand radius and polymer volume fraction can be used to estimate a mesh size.

In polymer networks, not only the obstruction effect, but also hydrodynamic drag forces play an important role in determining particle dynamics, and therefore models based purely on the obstruction effect are an oversimplification. Consider a nanoparticle that has to pass through a narrow pore: here, displacement of the constituent fluid is obviously more difficult than in bulk solution. Hydrodynamic approaches aim to predict the magnitude of the drag coefficient $f$ in the Einstein-Smoluchowski equation. Hydrodynamic models take into account the friction between 
nanoparticles and polymers, between nanoparticles and the solvent and between polymers and solvent.

Other forces present in colloidal systems such as electrostatic interactions and depletion forces (Asakura \& Oosawa, 1954) are generally ignored in diffusion models for rigid/fibrous networks. The use of particles that are not solvated, can lead to slippage and a violation of no-slip boundary conditions, and can compromise the validity of hydrodynamic models.

\subsection{Instrumental methods}

In order to prevent perturbation of the biopolymer network nanoparticles need to be dosed at low levels, typically at $0.1-1 \mathrm{wt} \%$. These low levels impose demands on the experimental method in terms of sensitivity. Furthermore, strong background signals of the matrix require selectivity with which nanoparticle signals can be detected. We will briefly discuss four suitable instrumental methods that meet these demands: fluorescence recovery after photobleaching (FRAP) microscopy, fluorescence correlation spectroscopy (FCS), single-particle tracking (SPT), and pulsed-field gradient nuclear magnetic resonance (PFG NMR) spectroscopy. An overview of these methods, including the time and length scales that can be probed and the required properties of nanoparticles, can be found in Table 2.3.

FRAP is a non-invasive method that can quantify the apparent diffusion coefficient of fluorescentlabeled particles as well as the mobile and immobile fractions. A laser is used to give a short, highintensity pulse to irreversibly bleach fluorescent labeled particles in a $\sim$ micrometer-size spot in the sample. Subsequently, a time trace of the in situ recovery of fluorescence is recorded, the recovery being due to non-bleached fluorescent particles outside the spot exchanging with bleached particles within the bleached spot. FRAP in conjunction with confocal laser scanning microscopy (CLSM) allows in situ selection of the bleaching spot. Micromolar fluorescent particle concentrations already suffice. Using models that account for the outflux of bleached probes and the influx of fluorescent probes, a diffusion coefficient can be extracted from image series recorded during fluorescence recovery. The accuracy of the FRAP method therefore ultimately depends on validity of the model used (Axelrod, Koppel, Schlessinger, Elson, \& Webb, 1976; Braeckmans, Peeters, Sanders, De Smedt, \& DeMeester, 2003). A single diffusion coefficient can be determined to with a precision of $\sim 1 \%$. In case of bimodal diffusion, diffusion coefficients can be extracted with an error of $\sim 10 \%$ (P. Jönsson, Jonsson, Tegenfeldt, \& Höök, 2008). 


\begin{tabular}{|c|c|c|c|}
\hline method (refs) & $\begin{array}{l}\text { time and length } \\
\text { scales }\end{array}$ & $\begin{array}{l}\text { nanoparticle } \\
\text { requirements }\end{array}$ & strengths and weaknesses \\
\hline $\begin{array}{l}\text { Fluorescence correlation } \\
\text { spectroscopy (FCS) } \\
\text { (Rathgeber, Beauvisage, } \\
\text { Chevreau, Willenbacher, \& } \\
\text { Oelschlaeger, 2009) }\end{array}$ & $\begin{array}{l}\text { time scale } \\
\text { lower: } 10^{-3} \mathrm{~ms} \\
\text { upper: up to } 10^{4} \mathrm{~ms} \\
\text { length scale } \\
10^{0} \mu \mathrm{m}\end{array}$ & $\begin{array}{l}\text {-Fluorescent } \\
\text {-Size }<10^{2} \mathrm{~nm}\end{array}$ & $\begin{array}{l}\text { +High time resolution } \\
\text { +Ensemble information } \\
\text { +Spatially resolved } \\
\text {-Optical transparency needed }\end{array}$ \\
\hline $\begin{array}{l}\text { Fluorescence recovery after } \\
\text { photobleaching (FRAP) } \\
\text { (Axelrod et al., 1976; Braeckmans } \\
\text { et al., 2003) }\end{array}$ & $\begin{array}{l}\text { time scale } \\
\text { time scales cannot be } \\
\text { directly probed } \\
\text { length scale } \\
10^{1}-10^{3} \mu \mathrm{m}\end{array}$ & -Fluorescent & $\begin{array}{l}\text { +Stationary fraction can be quantified } \\
\text { +Spatially resolved } \\
\text {-Only one single, long-term diffusion } \\
\text { coefficient can be determined }\end{array}$ \\
\hline $\begin{array}{l}\text { Pulsed-field gradient nuclear } \\
\text { magnetic resonance } \\
\text { spectroscopy (PFG NMR) } \\
\text { (Callaghan, 2011) }\end{array}$ & $\begin{array}{l}\text { time scale } \\
10^{0}-10^{3} \mathrm{~ms} \\
\text { length scale } \\
10^{1}-10^{6} \mu \mathrm{m} \\
\text { (diffusion-MRI) }\end{array}$ & $\begin{array}{l}\text {-Contains NMR-active } \\
\text { nuclei }\left(\text { such as }{ }^{1} \mathrm{H}\right) \text { with } \\
\text { liquidlike reorientation } \\
\text { dynamics }\end{array}$ & $\begin{array}{l}\text { +Ensemble information } \\
\text { + No optical transparency needed } \\
\text { +Propagator and diffusion coefficient } \\
\text { +Spatially resolved } \\
\text { +Signal separation of diffusing and } \\
\text { static compounds (DOSY, DRCOSY) } \\
\text {-Low sensitivity }\end{array}$ \\
\hline $\begin{array}{l}\text { Single particle tracking (SPT) } \\
\text { (Braeckmans et al., 2010; Filipe, } \\
\text { Hawe, \& Jiskoot, 2010) }\end{array}$ & $\begin{array}{l}\text { time scale } \\
\text { lower: } 1 \mathrm{~ms} \\
\text { upper: field-of-view } \\
\text { length scale } \\
10^{1}-10^{3} \mu \mathrm{m}\end{array}$ & $\begin{array}{l}\text {-High diffraction index, or } \\
\text { fluorescent } \\
\text {-No specific size limit }\end{array}$ & $\begin{array}{l}\text { +Information about individual } \\
\text { particles retained } \\
\text { +Spatially resolved } \\
\text {-Many observations required for } \\
\text { adequate statistics } \\
\text {-Possible depletion effects near cover } \\
\text { glass surface }\end{array}$ \\
\hline
\end{tabular}

Table 2.3: Overview of instrumental methods relevant for nanoparticle diffusometry. Indicated are the time and length scales of diffusion that are covered by the various methods, the demands that methods impose on nanoparticles and strength and weaknesses of the various methods.

FCS uses a confocal laser microscopy setup to monitor the fluctuations of fluorescence due to diffusion of fluorescently labeled particles inside a sub-micrometer confocal volume $\left(\sim 1 \mu \mathrm{m}^{3}\right)$ (Rathgeber et al., 2009). Brownian motion of fluorescent molecules in and out of the laser confocal volume will lead to fluorescence intensity fluctuations. From the fluorescence-intensity autocorrelation function, the particles' diffusion coefficients -also if the diffusion is multi-modalor the time-dependent mean-square displacements can be calculated (Koynov \& Butt, 2012; Rathgeber et al., 2009). FCS is a highly sensitive and selective technique that can be used on very small volumes, but careful calibration is required, because the volume of the laser focal point may vary depending on diffractive index. For imaging purposes, the method can be used in scanning mode (sFCS) (Petrášek, Ries, \& Schwille, 2010). FCS is sensitive and can measure self-diffusion over a wide range of observation times $\left(10^{-7}-10^{2} \mathrm{~s}\right)$, but ideally the samples should have a high degree of optical transparency. Like FRAP, FCS requires tracers functionalized with a fluorescent dye. An analogous method is dynamic light scattering (DLS), which measures the fluctuation of 
light that is scattered by diffusing particles. Recent developments in extending the lower particle size limit of DLS (Takahashi, Kato, \& Kinugasa, 2011) are difficult to exploit due to background scattering of other mobile species in the matrix, which complicates the interpretation of correlation curves.

SPT provides a direct visualization of individual diffusive trajectories of particles. So far, SPT has mainly been used for microrheology experiments, in which the micron-size tracer particles are visible under an optical microscope (Dickinson, 2011). Tracking of sub-micron particles, on the other hand, can be achieved by either detecting scattered light (Filipe et al., 2010), or fluorescence (Braeckmans et al., 2010; Mun et al., 2014). The observation of nanoparticles based on laser diffraction is only possible in systems where no other scattering elements are present. This is not a likely situation for most food systems, hence tracking of fluorescent particles is more promising for this purpose. The smallest fluorescent particles that have been tracked were $50 \mathrm{~nm}$ in diameter. A sensitive technique to detect motion of even smaller fluorescent particles is total internal reflection (TIRF), which has, however, a rather low sample penetration depth (Zareh, DeSantis, Kessler, Li, \& Wang, 2012). This may compromise representative sampling of diffusive trajectories in heterogeneous systems and limit the time window of the method. SPT is one of two methods discussed that allow direct assessment of the distribution of particle displacements in an ensemble, the other being PFG NMR. Most applications of SPT pertain to assessment of size, concentration and functionality of nanoparticles; only few examples have been given to probe local sub-micron network structure and dynamics (Mun et al., 2014). To our knowledge no application has appeared to assess sub-micron food structures.

PFG NMR is a method by which time-dependent displacements of molecular ensembles can be determined from the dephasing and rephasing of molecular spin magnetization by pulsed magnetic field gradients. These experiments can be implemented on any NMR spectrometer equipped with a field gradient coil.

PFG-based methods are particularly powerful if it comes to nanoparticle diffusometry in materials that are not optically transparent: NMR uses radiofrequency waves, which also penetrate optically non-transparent materials. The method is therefore well suited for nanoparticle diffusometry in optically non-transparent polysaccharide and protein matrices.

Using PFG NMR, the time-dependent diffusion coefficient can be determined in the $10^{\circ}-10^{3} \mathrm{~ms}$ range. By varying the observation time, the distance over which the network is probed can therefore be changed (Callaghan, 2011). In an experiment in which nanoparticles with different sizes (and hence, diffusion coefficients) are used to probe a network, the diffusion time can be adjusted to make sure that the mean-square displacement of different particles is identical. More specifically, PFG NMR directly detects the particles' van Hove self-correlation function. This means that multi-modal diffusion can be observed. Although no information about individual 
particles is retained, as is the case in SPT, the ensemble average of displacements over all particles in the sample is obtained (Callaghan, 2011). Only with PFG NMR, the diffusion of the ensemble of nanoparticles in a sample can be observed in a single experiment, which is a particular advantage for probing heterogeneous systems. Detection by NMR allows for separation signals on the basis of chemical shift or transverse relaxation time $T_{2}$, which offers the opportunity to resolve signals of nanoparticles and matrix (biopolymer network, solvent, solutes) (de Kort, van Duynhoven, Hoeben, Janssen, \& Van As, 2014; Lorén et al., 2009b). The frequency domain NMR experiment that separates molecular components on the basis of chemical shift is termed "diffusion-ordered spectroscopy" (DOSY), the time domain experiment that separates components on the basis of $T_{2}$ is termed "diffusion-relaxation correlation spectroscopy" (DRCOSY) (Callaghan, 2011). Diffusion measurements can also be performed in MRI (imaging) mode. This allows the determination of local diffusion coefficients on the micron scale, with a field of view of $10^{-2}-10^{-1}$ $\mathrm{m}$, depending on the setup. This is what makes PFG NMR a truly multi-length-scale method. With an MRI setup, also the anisotropic diffusion tensor can be resolved (Future Trends) (Callaghan, 2011).

\subsection{Applications in food science}

In Table 2.4, a range of recent applications of nanoparticle diffusometry in food biopolymer networks has been collected.

Early contributions introducing the concepts of nanoparticle diffusometry in biopolymer networks stem from the 80s. For example, Brown et al. used PFG NMR to study self-diffusion of PEG nanoparticles in dextran solutions, and of small molecules (ethylene glycol, crown ether) in cellulose dispersions (Brown \& Stilbs, 1983; Brown, Stilbs, \& Lindström, 1984). The concept of inferring information about the biopolymer network from the diffusion of nanoparticles was introduced here, and simple obstruction effect-based models were used to describe the data. At the time of these studies, however, models of diffusion that included hydrodynamic interactions were not available. Also, no conceptual distinction was made between diffusometry in solutions of flexible polymers and rigid/fibrous networks or gels, which relates to the fact that the microrheological experiment originated only a decade later. The first steps toward the use of FRAP for nanoparticle diffusometry stems from the early 90s; Significant work includes that of Furukawa et al., describing nanoparticle diffusion in dextran solutions, although this study was not directly related to foods (Furukawa, Arauz-Lara, \& Ware, 1991). The work already includes a critical discussion of the meaning of nanoparticle diffusometric data in polymer solutions using reptation and hydrodynamic concepts. 


\begin{tabular}{|c|c|c|c|c|}
\hline biopolymer network & instrumental method & $\begin{array}{l}\text { nanoparticles (hydrodynamic } \\
\text { radius } R_{h} \text { or molecular weight } M_{w} \text { ) }\end{array}$ & model class & references \\
\hline agarose & $\begin{array}{l}\text { refractive-index method } \\
\text { (see in ref.) }\end{array}$ & $\mathrm{PEG}^{\mathrm{a}}\left(M_{\mathrm{w}}=0.2,0.6,1,10 \mathrm{kDa}\right)$ & $\begin{array}{l}\text { power-law, obstruction } \\
\text { effect }\end{array}$ & (Weng, Liang, Zhang, Zhang, \& Xu, 2005) \\
\hline alginate & $\begin{array}{l}\text { release kinetics (see (Favre } \\
\text { et al., 2001)), PFG NMR }\end{array}$ & $\begin{array}{l}\text { star-shaped }\left(M_{\mathrm{w}}=24 \mathrm{kDa}\right) \text { and } \\
\text { linear }\left(M_{\mathrm{w}}=80-750 \mathrm{kDa}\right) \text { PEG, } \\
\text { dendrimers }\left(R_{\mathrm{h}}=2-8 \mathrm{~nm}\right)\end{array}$ & $\begin{array}{l}\text { hydrodynamics modeling, } \\
\text { obstruction effect }\end{array}$ & $\begin{array}{l}\text { (Baldursdóttir, Kjøniksen, \& Nyström, 2006; } \\
\text { Bernin et al., 2011; Favre et al., 2001) }\end{array}$ \\
\hline$\beta$-lactoglobulin & $\begin{array}{l}\text { CLSM }^{\mathrm{c}} \text { FRAP }^{\mathrm{d}}, \text { PFG } \\
\text { NMR }\end{array}$ & $\begin{array}{l}\text { fluorescent-labeled dextran }\left(R_{\mathrm{h}}=\right. \\
5.5,23 \text { and } 50 \mathrm{~nm}) \text {, dextran }\left(R_{\mathrm{h}}=8,\right. \\
11,23 \mathrm{~nm})\end{array}$ & model-free interpretation & $\begin{array}{l}\text { (Balakrishnan, Nicolai, \& Durand, 2012; } \\
\text { Croguennoc, Nicolai, Kuil, \& Hollander, 2001) }\end{array}$ \\
\hline $\begin{array}{l}\text { casein micelles and } \\
\text { caseinate }\end{array}$ & PFG NMR & $\begin{array}{l}\text { linear PEG }\left(M_{\mathrm{w}}=1-600 \mathrm{kDa}\right) \text {, } \\
\text { dendrimers }\left(R_{\mathrm{h}}=2-6 \mathrm{~nm}\right)\end{array}$ & $\begin{array}{l}\text { obstruction effect, power } \\
\text { law }\end{array}$ & $\begin{array}{l}\text { (Colsenet, Mariette, \& Söderman, 2006a; } \\
\text { Colsenet, Söderman, \& Mariette, 2005b; 2005a; } \\
\text { Le Feunteun \& Mariette, 2007; 2008a; 2008b; } \\
\text { Le Feunteun, Ouethrani, \& Mariette, 2012; } \\
\text { Salami, Rondeau-Mouro, Barhoum, van } \\
\text { Duynhoven, \& Mariette, 2014; Salami, } \\
\text { Rondeau-Mouro, van Duynhoven, \& Mariette, } \\
\text { 2013a; 2013b) }\end{array}$ \\
\hline curdlan & PFG NMR & $\begin{array}{l}\text { PEG }\left(M_{\mathrm{w}}=1,3 \mathrm{kDa}\right), \text { micelles }\left(R_{\mathrm{h}}=\right. \\
41 \mathrm{~nm})\end{array}$ & $\begin{array}{l}\text { models by Cukier (Cukier, } \\
\text { 1984) and Petit (Petit, } \\
\text { Roux, Zhu, \& Macdonald, } \\
\text { 1996) }\end{array}$ & (Kwak \& Lafleur, 2003b) \\
\hline collagen & (line-)FRAP & FITC-dextran $\left(R_{\mathrm{h}}=20 \mathrm{~nm}\right)$ & $\begin{array}{l}\text { model for anisotropic } \\
\text { diffusion in aligned-fiber } \\
\text { systems }\end{array}$ & $\begin{array}{l}\text { (Stylianopoulos, Diop-Frimpong, Munn, \& Jain, } \\
\text { 2010) }\end{array}$ \\
\hline dextran & FRAP, PFG NMR & $\begin{array}{l}\text { fluorescein-labeled dextran }\left(M_{\mathrm{w}}=\right. \\
40,150 \mathrm{kDa}) \text {, fluorescein-labeled } \\
\text { polystyrene spheres }\left(\mathrm{R}_{\mathrm{h}}=19 \mathrm{~nm}\right) \text {, } \\
\text { and SPS, CPC, and Triton X-100 } \\
\text { micelles }\left(R_{\mathrm{h}}=17,2953 \mathrm{~nm}\right)\end{array}$ & $\begin{array}{l}\text { discussion of } \\
\text { hydrodynamic models, } \\
\text { scaling laws }\end{array}$ & (Furukawa et al., 1991; Kwak \& Lafleur, 2003a) \\
\hline gelatin & CLSM FRAP & FITC $^{\mathrm{e}}$-dextran $\left(R_{\mathrm{h}}=6\right.$ and $\left.34 \mathrm{~nm}\right)$ & model-free interpretation & (Hagman, Lorén, \& Hermansson, 2010) \\
\hline
\end{tabular}




\begin{tabular}{|c|c|c|c|c|}
\hline guar galactomannan & FRAP & $\begin{array}{l}\text { FITC-dextran }\left(M_{\mathrm{w}}=20,40,71\right. \\
\mathrm{kDa}) \text {, dextran and dendrimers, } R_{\mathrm{h}}= \\
1-20 \mathrm{~nm}\end{array}$ & $\begin{array}{l}\text { models by Cukier (Cukier, } \\
\text { 1984), Langevin } \\
\text { (Langevin \& Rondelez, } \\
\text { 1978) and Altenberger } \\
\text { (Altenberger, Tirrell, \& } \\
\text { Dahler, 1986) }\end{array}$ & $\begin{array}{l}\text { (Burke, Park, Srinivasarao, \& Khan, 2000; } \\
\text { Cheng et al., 2002) }\end{array}$ \\
\hline$\kappa$-carrageenan & PFG NMR, CLSM FRAP & $\begin{array}{l}\text { PAMAM and PEGylated, }{ }^{19} \mathrm{~F}- \\
\text { labeled PPI dendrimers }\left(R_{\mathrm{h}}=2-8\right. \\
\mathrm{nm}), \text { FITC-dextran }(10,500 \mathrm{kDa})\end{array}$ & model-free interpretation & $\begin{array}{l}\text { (de Kort et al., 2014; Hagman, Lorén, \& } \\
\text { Hermansson, 2012; Lorén et al., 2009b) }\end{array}$ \\
\hline konjac glucomannan & FRAP & $\begin{array}{l}\text { FITC-dextran }\left(M_{\mathrm{w}}=77,130,511,\right. \\
2000 \mathrm{kDa})\end{array}$ & power law & (Alvarez-Manceñido et al., 2006) \\
\hline pig gastric mucin & PFG NMR, DLS & $\begin{array}{l}\text { linear PEG }\left(M_{\mathrm{w}}=1,6,59,716\right. \\
\mathrm{kDa}) \text {, polysorbate } 80\left(R_{\mathrm{h}}=6 \mathrm{~nm}\right), \\
\text { polystyrene beads }(R=54 \mathrm{~nm})\end{array}$ & $\begin{array}{l}\text { hydrodynamics, power } \\
\text { law, model-free } \\
\text { interpretation }\end{array}$ & $\begin{array}{l}\text { (Celli et al., 2005; Lafitte, Söderman, } \\
\text { Thuresson, \& Davies, 2007a; Lafitte, Thuresson, } \\
\text { Jarwoll, \& Nydén, 2007b) }\end{array}$ \\
\hline starch & FRAP & $\operatorname{dextran}\left(M_{\mathrm{w}}=70,250 \mathrm{kDa}\right)$ & power law & (Perry, Fitzgerald, \& Gilbert, 2006) \\
\hline $\begin{array}{l}\text { ultrafiltrated milk model } \\
\text { cheese }\end{array}$ & CLSM FRAP & $\begin{array}{l}\text { FITC-dextran }\left(R_{\mathrm{h}}=2-24 \mathrm{~nm}\right) \text { and } \\
\text { FITC-dairy proteins }\left(R_{\mathrm{h}}=2-4 \mathrm{~nm}\right)\end{array}$ & model-free interpretation & $\begin{array}{l}\text { (Floury, Madec, Waharte, Jeanson, \& Lortal, } \\
\text { 2012; Silva et al., 2013) }\end{array}$ \\
\hline whey protein & PFG NMR & linear PEG $\left(M_{\mathrm{w}}=1,9,82 \mathrm{kDa}\right)$ & power law & (Colsenet, Söderman, \& Mariette, 2006b) \\
\hline $\begin{array}{l}\text { whey protein isolate- } \\
\text { gellan gum, micro- } \\
\text { phase-separated system }\end{array}$ & PFG NMR, FRAP CLSM & $\begin{array}{l}\text { alpha-lactalbumin }\left(R_{\mathrm{h}}=2 \mathrm{~nm}\right) \\
\text { FITC-dextran }\left(M_{\mathrm{w}}=10 \mathrm{kDa}\right)\end{array}$ & $\begin{array}{l}\text { Lattice Boltzmann } \\
\text { simulations in model gel }\end{array}$ & (Wassén et al., 2014) \\
\hline
\end{tabular}

Table 2.4: Overview of applications of nanoparticle diffusometry in food related materials. The table includes systems studied, and instrumental methods and nanoparticles used. Also modeling approaches are indicated, which can be either based on the obstruction effect (in case of rigid nanoparticles, cf. also Table 2.2) or on power laws (in case of flexible nanoparticles). It is also indicated if a model-free interpretation was made, a new model was proposed or models were used that have not been discussed in this paper (because they do not model nanoparticle diffusion in terms of structural network parameters). 
One of the first applications in food biopolymer networks is a FRAP study on a guar galactomannan model system using FITC-dextran complexes (Burke et al., 2000). The interpretation of the diffusometric data related to the crossover point between Zimm and reptation dynamics in dilute and concentrated polymer solutions, respectively, as described e.g. by Nydén and Söderman (Nydén \& Söderman, 1998). Soon thereafter, Favre et al. published the first PFG NMR study of PEG diffusion in alginate gels, applying these concepts to diffusion of flexible probes in dilute versus concentrated systems (Favre et al., 2001). We have described this approach, which is still current, in the section about diffusion models for flexible nanoparticles. Over the following decade, many more studies in both polysaccharide and protein systems would follow, with the FRAP and PFG NMR methods as workhorses. During this period, the diversity in nanoparticles increased. In 2003, Kwak and Lafleur used spherical particles (micelles) for the first time for the characterization of a biopolymer network by PFG NMR (Kwak \& Lafleur, 2003b). The first foods-related diffusion study using dendrimers would follow some years later in 2009 (Lorén et al., 2009b). We will now describe the most important diffusometry studies in polysaccharide and protein networks.

\subsubsection{Characterization of polysaccharide networks}

Baldursdóttir studied the effect of photo-degradation of alginate gels on diffusion of embedded PEG molecules and was able to infer microstructural changes within the gels from the changes in diffusive behavior of nanoparticles (Baldursdóttir et al., 2006). Bernin et al. characterized these systems using a combination of transmission electron microscopy (TEM) and nanoparticle diffusometry. They calculated alginate polymer-strand radii from the reduced diffusion coefficient of dendrimer nanoparticles and validated this information against TEM micrographs. They found that in some gels, a submicron network was present that TEM was unable to visualize (Bernin et al., 2011). k-Carrageenan gels have also been analyzed with the combination of TEM and nanoparticle diffusometry. Using TEM, the effects of polymer concentration and different salt conditions on microstructure were visualized. Not only polymer density was found to affect nanoparticle self-diffusion; particularly salt conditions led to interesting submicron-scale changes in network heterogeneity, which were reflected by changes in diffusion behavior of the nanoparticles. In some cases, bi-modal diffusion was observed, tentatively due to the presence of two different micro-domains within the gel morphology (de Kort et al., 2014; Lorén et al., 2009b).

\subsubsection{Characterization of protein networks}

Croguennoc and later Balakrishnan studied nanoparticle diffusion during and after gelation of $\beta$ lactoglobulin, a globular protein that is the major component of whey, and which can form gels through heating. These studies showed that nanoparticles could be used to assess the kinetics of the gelation process, and the profound effects of protein and salt concentrations on void sizes in resulting gels, which was reflected by changes in nanoparticle diffusion coefficients (Balakrishnan 
et al., 2012; Croguennoc et al., 2001).

In order to see the effect of fiber alignment, aligned collagen systems were studied using FITClabeled dextran molecules (Stylianopoulos et al., 2010). A model was derived in order to understand the relationship between collagen fiber radius and particle diffusion anisotropy. It was concluded that material anisotropy could only be observed with nanoparticles sufficiently large with respect to the aligned-fiber radius. In this study, anisotropy was indeed observed when the particle-fiber radius ratio was between 1 and 2, whereas in an earlier study in which the ratio was $\ll 1$, isotropic diffusion was observed.

Mariette et al. have intensively studied coagulation of milk proteins into casein micelle gels using the nanoparticle diffusometry approach. Their initial work focused on the diffusion of flexible PEG molecules through the networks (Colsenet, Mariette, \& Söderman, 2006a; Colsenet, Söderman, \& Mariette, 2005a; 2005b; Le Feunteun \& Mariette, 2007; 2008a). More recently, dendrimer particles have been used for the same purpose (Salami et al., 2014; Salami, Rondeau-Mouro, van Duynhoven, \& Mariette, 2013b). Le Feunteun et al. deployed NMR diffusometry to continuously monitor the changes in the structure during the coagulation process itself (Le Feunteun \& Mariette, 2008b). The PEG-probe based approach was also used for the characterization of whey protein gels (Colsenet, Söderman, \& Mariette, 2006b).

In a more recent approach, Le Feunteun et al. and Salami et al. followed diffusion of casein proteins through porous micellar aggregates. Diffusion was described by the Speedy model for hard-sphere diffusion through voids in a system of closely packed spheres. This way, the voluminosity of the casein micelles could be derived (Le Feunteun et al., 2012; Salami, RondeauMouro, van Duynhoven, \& Mariette, 2013a).

In very recent work, Floury and Silva have studied the diffusion of solutes and nanoparticles through a model cheese, based on ultra-filtrated milk. Understanding mass transport in cheese is relevant, because diffusion of salt, moisture and metabolites is very important for the final quality of cheese. Particularly the diffusion of nanoscopic particles in cheese had not yet been studied. This example illustrates how the step from abstract model systems to more complex and heterogeneous systems is being taken (Floury et al., 2012; Silva et al., 2013).

\subsection{Future trends}

From the food-related application areas of nanoparticle diffusometry that have been explored in the last decade, it has become clear that particularly rigid biopolymer networks (gels) that are structured on the submicron scale lend themselves for characterization using nanoparticle diffusometry. Using obstruction models it is possible to derive quantitative structural parameters in a straightforward manner, which is a considerable advantage over current microscopic methods. We will now sketch some perspectives for the characterization of rigid as well as flexible polymer networks. We will also discuss the possibility to use nanoparticle diffusometry to measure network anisotropy and the scope of novel nanoparticle designs. 
Design of functionalized nanoparticles. Obstruction models generally assume the diffusing species to be rigid and spherical. In this light, dendrimers are near-ideal diffusional nanoparticles because they have a fractal index close to that of solid spheres and are essentially monodisperse. Their size can be varied in several discrete steps up to several nanometers. An additional attractive feature is their well-defined number of end groups, which in principle can be readily functionalized. This way, their surface chemistry (including surface charge) can be fine-tuned to specific applications, e.g. by PEGylation in order to better satisfy the non-sticky condition. In case neutral particles are required, their charged surface groups should be functionalized with neutral end groups. For example, the commercially available poly(amido amine) (PAMAM) and poly(propylene imine) (PPI) dendrimers have primary amine end groups and can therefore carry charges (Boas, Christensen, \& Heegaard, 2006).

Additionally, dendrimers can be functionalized with labels, such as fluorescent dyes to allow detection by FRAP, FCS and SPT. We recently reported a design of (covalently) PEGylated, charge neutral, ${ }^{19} \mathrm{~F}$-labeled PPI dendrimers for (background-free) observation by PFG NMR (de Kort et al., 2014). A limitation of dendrimers is their limited size range (max. diameter $\sim 10 \mathrm{~nm}$ ) and relatively high cost. Bourouina et al. recently circumvented this drawback by using complex coacervate (polyion) micelles as a chassis for designing and manufacturing larger and functionalized diffusional nanoparticles (Bourouina, Cohen Stuart, \& Kleijn, 2014).

Sub-micron heterogeneity. Obstruction-effect based modeling of diffusional behavior of nanoparticles can yield quantitative network descriptors in a straightforward manner. These models assume that a single diffusion coefficient is found for the diffusing species. Often, systems are not homogeneous on the sub-micron-scale, meaning that on time scales at which mean particle displacement is on the order of the length scale of the heterogeneities, a spatial distribution of nanoparticle diffusion coefficients will be present. The spatial distribution arises from the different microenvironments through which the nanoparticles move. In the long run, if nanoparticles can move between microenvironments, a single, average "terminal" diffusion coefficient will be observed (central limit theorem, cf. Table 2.1). The observation time at which the distribution of diffusion coefficients disappears, and only a single nanoparticle diffusion coefficient remains, is therefore directly related to the size of the heterogeneities in the structure. If no exchange between domains is possible, increasing diffusion time will not lead to a single diffusion coefficient, and the distribution in particle displacements will be related to domain sizes.

Micron-size tracer particles have also been used to assess heterogeneity of agarose (Valentine et al., 2001), acid milk gels (Cucheval, Vincent, Hemar, Otter, \& Williams, 2009) and phase-separated systems (Dickinson, Murray, \& Moschakis, 2007).

Nanoparticles have been used to observe heterogeneity in $\kappa$-carrageenan gels. (de Kort et al., 2014; Lorén et al., 2009b) Multi-modal diffusion can be observed using PFG NMR, since this method measures the ensemble-average propagator directly. 
PFG NMR offers a broader perspective on gel heterogeneity, because the diffusion-observation time can be varied, opening the opportunity to monitor the changes in diffusion coefficients as a function time. This would allow investigation of the nature of the heterogeneities, that is, whether or not exchange between the domains takes place, and derivation of structural parameters. Diffusion-diffusion exchange spectroscopy (DEXSY) allows a more direct assessment of diffusive exchange between two nanoparticle populations (Callaghan, 2011).

Despite its promise, we have not been able to find any food science related studies that follow this approach, possibly because of the limited time-observation window of NMR $\left(10^{0}-10^{3} \mathrm{~ms}\right)$.

\subsection{Conclusion}

In food science, nanoparticle diffusometry has been mainly used for the characterization of heterogeneous and rigid polymer networks. The theoretical framework for interpreting diffusion data is becoming more elaborate and mature. Two different modeling approaches can be distinguished. (1) Models that predict the diffusion of rigid, spherical nanoparticles through polymer gels. These models are mainly based on the notion of the obstruction effect and can be used to predict structural network descriptors, such as mesh size and average polymer strand radii. (2) Models that describe the diffusion of flexible nanoparticles such as polymer chains in crowded systems, based on arguments from polymer theory. These models assume that the diffusion of nanoparticles changes from Zimm-like to reptation-like, depending on the degree of crowding. By varying the molecular weight of the nanoparticle, information about the microstructure (mesh size) can be obtained.

This review aimed at summarizing the theoretical framework and methodological approaches for nanoparticle diffusometry. The method is already being used to characterize more advanced food matrices, such as model cheeses (Silva et al., 2013). More exciting developments are expected, such as functionalized nanoparticle designs. Furthermore we expect to see more work relating to the observation of changing diffusion coefficients under dynamical conditions. 


\section{References}

Altenberger, A. R., Tirrell, M., \& Dahler, J. S. (1986). Hydrodynamic screening and particle dynamics in porous media, semidilute polymer solutions and polymer gels. The Journal of Chemical Physics, 84(9), 5122-5130. http://doi.org/10.1063/1.450665

Alvarez-Manceñido, F., Braeckmans, K., De Smedt, S. C., Demeester, J., Landin, M., \& Martínez-Pacheco, R. (2006). Characterization of diffusion of macromolecules in konjac glucomannan solutions and gels by fluorescence recovery after photobleaching technique. International Journal of Pharmaceutics, 316(1-2), 37-46. http://doi.org/10.1016/j.ijpharm.2006.02.029

Amsden, B. (1998a). Solute diffusion in hydrogels. Polymer Gels and Networks, 6(1), 13-43. http://doi.org/10.1016/S0966-7822(97)00012-9

Amsden, B. (1998b). Solute Diffusion within Hydrogels. Mechanisms and Models. Macromolecules, 31(23), 8382-8395. http://doi.org/10.1021/ma980765f

Asakura, S., \& Oosawa, F. (1954). On Interaction between Two Bodies Immersed in a Solution of Macromolecules. The Journal of Chemical Physics, 22(7), 1255-1256. http://doi.org/10.1063/1.1740347

Axelrod, D., Koppel, D. E., Schlessinger, J., Elson, E., \& Webb, W. W. (1976). Mobility Measurement by Analysis of Fluorescence Photobleaching Recovery Kinetics. Biophysical Journal, 16(9), 1055-1069. http://doi.org/10.1016/S0006-3495(76)85755-4

Babu, S., Gimel, J.-C., \& Nicolai, T. (2008). Tracer diffusion in colloidal gels. The Journal of Chemical Physics, 112(3), 743-748. http://doi.org/10.1021/jp076342

Balakrishnan, G., Nicolai, T., \& Durand, D. (2012). Relation between the gel structure and the mobility of tracers in globular protein gels. Journal of Colloid and Interface Science, 388(1), 293-299. http://doi.org/10.1016/j.jcis.2012.08.032

Baldursdóttir, S. G., Kjøniksen, A.-L., \& Nyström, B. (2006). The effect of riboflavin-photoinduced degradation of alginate matrices on the diffusion of poly(oxyethylene) probes in the polymer network. European Polymer Journal, 42(11), 3050-3058. http://doi.org/10.1016/j.eurpolymj.2006.08.011

Bernin, D., Goudappel, G.-J., van Ruijven, M., Altskär, A., Ström, A., Rudemo, M., et al. (2011). Microstructure of polymer hydrogels studied by pulsed field gradient NMR diffusion and TEM methods. Soft Matter, 7(12), 5711-5716. http://doi.org/10.1039/c1sm05070b

Boas, U., Christensen, J. B., \& Heegaard, P. M. H. (2006). Dendrimers: design, synthesis and chemical properties. Journal of Materials Chemistry, 16(38), 3785-3798. http://doi.org/10.1039/B611813P

Bourouina, N., Cohen Stuart, M. A., \& Kleijn, J. M. (2014). Complex coacervate core micelles as diffusional nanoprobes. Soft Matter, 10(2), 320-331. http://doi.org/10.1039/c3sm52245h

Braeckmans, K., Buyens, K., Naeye, B., Vercauteren, D., Deschout, H., Raemdonck, K., et al. (2010). Advanced fluorescence microscopy methods illuminate the transfection pathway of nucleic acid nanoparticles. Journal of Controlled Release, 148(1), 69-74. http://doi.org/10.1016/j.jconrel.2010.08.029

Braeckmans, K., Peeters, L., Sanders, N. N., De Smedt, S. C., \& DeMeester, J. (2003). Three-Dimensional Fluorescence Recovery after Photobleaching with the Confocal Scanning Laser Microscope. Biophysical Journal, 85(4), 2240-2252. http://doi.org/10.1016/S0006-3495(03)74649-9

Brochard Wyart, F., \& de Gennes, P. G. (2000). Viscosity at small scales in polymer melts. European Physical Journal E, 1(1), 93-97. http://doi.org/10.1007/s101890050011

Brown, W., \& Stilbs, P. (1983). Self-diffusion of poly(ethylene oxide) in aqueous dextran solutions measured using FT-pulsed field gradient n.m.r. Polymer, 24(2), 188-192. http://doi.org/10.1016/00323861(83)90131-3

Brown, W., Stilbs, P., \& Lindström, T. (1984). Self-diffusion of small molecules in cellulose gels using FTpulsed field gradient NMR. Journal of Applied Polymer Science, 29(3), 823-827. http://doi.org/10.1002/app.1984.070290310 
Burke, M. D., Park, J. O., Srinivasarao, M., \& Khan, S. A. (2000). Diffusion of Macromolecules in Polymer Solutions and Gels: A Laser Scanning Confocal Microscopy Study. Macromolecules, 33(20), 75007507. http://doi.org/10.1021/ma0007861

Cai, L.-H., Panyukov, S., \& Rubinstein, M. (2011). Mobility of Nonsticky Nanoparticles in Polymer Liquids. Macromolecules, 44(19), 7853-7863. http://doi.org/10.1021/ma201583q

Callaghan, P. T. (2011). Translational Dynamics \& Magnetic Resonance. New York: Oxford University Press.

Carvajal-Rondanelli, P. A., \& Lanier, T. C. (2010). Diffusion of Active Proteins into Fish Meat To Minimize Proteolytic Degradation. Journal of Agricultural and Food Chemistry, 58(9), 5300-5307. http://doi.org/10.1021/jf903580t

Celli, J., Gregor, B., Turner, B., Afdhal, N. H., Bansil, R., \& Erramilli, S. (2005). Viscoelastic Properties and Dynamics of Porcine Gastric Mucin. Biomacromolecules, 6(3), 1329-1333. http://doi.org/10.1021/bm0493990

Cheng, Y., Prud'homme, R. K., \& Thomas, J. L. (2002). Diffusion of Mesoscopic Probes in Aqueous Polymer Solutions Measured by Fluorescence Recovery after Photobleaching. Macromolecules, 35(21), 81118121. http://doi.org/10.1021/ma0107758

Clague, D. S., \& Phillips, R. J. (1996). Hindered diffusion of spherical macromolecules through dilute fibrous media. Physics of Fluids, 8(7), 1720-1731. http://doi.org/10.1063/1.868884

Colsenet, R., Mariette, F., \& Söderman, O. (2006a). NMR study of the polyethyleneglycols diffusion in casein suspensions and gels. Presented at the ISFRS 2006, 4th International symposium on food rheology and structure.

Colsenet, R., Söderman, O., \& Mariette, F. (2005a). Diffusion of polyethyleneglycols in casein solutions and gels as studied by pulsed field gradient NMR. Magnetic Resonance Imaging, 23(2), 347-348. http://doi.org/10.1016/j.mri.2004.11.049

Colsenet, R., Söderman, O., \& Mariette, F. (2005b). Effect of Casein Concentration in Suspensions and Gels on Poly(ethylene glycol)s NMR Self-Diffusion Measurements. Macromolecules, 38(22), 9171-9179. http://doi.org/10.1021/ma051294h

Colsenet, R., Söderman, O., \& Mariette, F. (2006b). Pulsed Field Gradient NMR Study of Poly(ethylene glycol) Diffusion in Whey Protein Solutions and Gels. Macromolecules, 39(3), 1053-1059. http://doi.org/10.1021/ma0519922

Croguennoc, P., Nicolai, T., Kuil, M. E., \& Hollander, J. G. (2001). Self-diffusion of Native Proteins and Dextran in Heat-set Globular Protein Gels. The Journal of Chemical Physics, 105(24), 5782-5788. http://doi.org/10.1021/jp0043726

Cucheval, A. S. B., Vincent, R. R., Hemar, Y., Otter, D., \& Williams, M. A. K. (2009). Multiple particle tracking investigations of acid milk gels using tracer particles with designed surface chemistries and comparison with diffusing wave spectroscopy studies. Langmuir, 25(19), 11827-11834. http://doi.org/10.1021/la901424w

Cukier, R. I. (1984). Diffusion of Brownian spheres in semidilute polymer solutions. Macromolecules, 17(2), 252-255. http://doi.org/10.1021/ma00132a023

de Gennes, P. G. (1979). Scaling Concepts in Polymer Physics. Ithaca, NY: Cornell University Press.

de Kort, D. W., van Duynhoven, J. P. M., Hoeben, F. J. M., Janssen, H. M., \& Van As, H. (2014). NMR nanoparticle diffusometry in hydrogels: enhancing sensitivity and selectivity. Analytical Chemistry, 86(18), 9229-9235. http://doi.org/10.1021/ac502211q

Dickinson, E. (2011). Food colloids research: Historical perspective and outlook. Advances in Colloid and Interface Science, 165(1), 7-13. http://doi.org/10.1016/j.cis.2010.05.007

Dickinson, E., Murray, B. S., \& Moschakis, T. (2007). Particle tracking as a probe of microrheology in food colloids. In Food Colloids (pp. 305-318). Cambridge: Royal Society of Chemistry. http://doi.org/10.1039/9781847557698-00303 
Favre, E., Leonard, M., Laurent, A., \& Dellacherie, E. (2001). Diffusion of polyethyleneglycols in calcium alginate hydrogels. Colloids and Surfaces A, 194(1), 197-206. http://doi.org/10.1016/S09277757(01)00789-0

Filipe, V., Hawe, A., \& Jiskoot, W. (2010). Critical Evaluation of Nanoparticle Tracking Analysis (NTA) by NanoSight for the Measurement of Nanoparticles and Protein Aggregates. Pharmaceutical Research, 27(5), 796-810. http://doi.org/10.1007/s11095-010-0073-2

Floury, J., Madec, M. N., Waharte, F., Jeanson, S., \& Lortal, S. (2012). First assessment of diffusion coefficients in model cheese by fluorescence recovery after photobleaching (FRAP). Food Chemistry, 133(2), 551-556. http://doi.org/10.1016/j.foodchem.2012.01.030

Furukawa, R., Arauz-Lara, J. L., \& Ware, B. R. (1991). Self-diffusion and probe diffusion in dilute and semidilute aqueous solutions of dextran. Macromolecules, 24(2), 599-605. http://doi.org/10.1021/ma00002a039

Gottwald, A., Creamer, L. K., Hubbard, P. L., \& Callaghan, P. T. (2005). Diffusion, relaxation, and chemical exchange in casein gels: a nuclear magnetic resonance study. The Journal of Chemical Physics, 122(3), 34506. http://doi.org/10.1063/1.1825383

Gupta, A. K., \& Gupta, M. (2005). Synthesis and surface engineering of iron oxide nanoparticles for biomedical applications. Biomaterials, 26(18), 3995-4021. http://doi.org/10.1016/j.biomaterials.2004.10.012

Hagman, J., Lorén, N., \& Hermansson, A.-M. (2010). Effect of Gelatin Gelation Kinetics on Probe Diffusion Determined by FRAP and Rheology. Biomacromolecules, 11(12), 3359-3366. http://doi.org/10.1021/bm1008487

Hagman, J., Lorén, N., \& Hermansson, A.-M. (2012). Probe diffusion in $\kappa$-carrageenan gels determined by fluorescence recovery after photobleaching. Food Hydrocolloids, 29(1), 106-115. http://doi.org/10.1016/j.foodhyd.2012.02.010

Johansson, L., Skantze, U., \& Loefroth, J. E. (1991). Diffusion and interaction in gels and solutions. 2. Experimental results on the obstruction effect. Macromolecules, 24(22), 6019-6023. http://doi.org/10.1021/ma00022a018

Johnson, E. M., Berk, D. A., Jain, R. K., \& Deen, W. M. (1996). Hindered diffusion in agarose gels: test of effective medium model. Biophysical Journal, 70(2), 1017-1023. http://doi.org/10.1016/S00063495(96)79645-5

Jönsson, P., Jonsson, M. P., Tegenfeldt, J. O., \& Höök, F. (2008). A Method Improving the Accuracy of Fluorescence Recovery after Photobleaching Analysis. Biophysical Journal, 95(11), 5334-5348. http://doi.org/10.1529/biophysj.108.134874

Kohli, I., \& Mukhopadhyay, A. (2012). Diffusion of Nanoparticles in Semidilute Polymer Solutions: Effect of Different Length Scales. Macromolecules, 45(15), 6143-6149. http://doi.org/10.1021/ma301237r

Koynov, K., \& Butt, H.-J. (2012). Fluorescence correlation spectroscopy in colloid and interface science. Current Opinion in Colloid \& Interface Science, 17(6), 377-387. http://doi.org/10.1016/j.cocis.2012.09.003

Kvarnström, M., Westergård, A., Lorén, N., \& Nydén, M. (2009). Brownian dynamics simulations in hydrogels using an adaptive time-stepping algorithm. Physical Review E, 79(1), 016102. http://doi.org/10.1103/PhysRevE.79.016102

Kwak, S., \& Lafleur, M. (2003a). NMR Self-Diffusion of Molecular and Macromolecular Species in Dextran Solutions and Gels. Macromolecules, 36(9), 3189-3195. http://doi.org/10.1021/ma0213605

Kwak, S., \& Lafleur, M. (2003b). Self-diffusion of macromolecules and macroassemblies in curdlan gels as examined by PFG-SE NMR technique. Colloids and Surfaces A, 221(1-3), 231-242. http://doi.org/10.1016/S0927-7757(03)00145-6

Lafitte, G., Söderman, O., Thuresson, K., \& Davies, J. (2007a). PFG-NMR diffusometry: A tool for investigating the structure and dynamics of noncommercial purified pig gastric mucin in a wide range of 
concentrations. Biopolymers, 86(2), 165-175. http://doi.org/10.1002/bip.20717

Lafitte, G., Thuresson, K., Jarwoll, P., \& Nydén, M. (2007b). Transport Properties and Aggregation Phenomena of Polyoxyethylene Sorbitane Monooleate (Polysorbate 80) in Pig Gastrointestinal Mucin and Mucus. Langmuir, 23(22), 10933-10939. http://doi.org/10.1021/la701081s

Langevin, D., \& Rondelez, F. (1978). Sedimentation of large colloidal particles through semidilute polymer solutions. Polymer, 19(8), 875-882. http://doi.org/10.1016/0032-3861(78)90191-X

Lauffer, M. A. (1961). Theory of diffusion in gels. Biophysical Journal, 1(3), 205-213. http://doi.org/10.1016/S0006-3495(61)86884-7

Le Feunteun, S., \& Mariette, F. (2007). Impact of Casein Gel Microstructure on Self-Diffusion Coefficient of Molecular Probes Measured by ${ }^{1} \mathrm{H}$ PFG-NMR. Journal of Agricultural and Food Chemistry, 55(26), 10764-10772. http://doi.org/10.1021/jf071982v

Le Feunteun, S., \& Mariette, F. (2008a). Effects of Acidification with and without Rennet on a Concentrated Casein System: A Kinetic NMR Probe Diffusion Study. Macromolecules, 41(6), 2079-2086. http://doi.org/10.1021/ma702248z

Le Feunteun, S., \& Mariette, F. (2008b). PFG-NMR Techniques Provide a New Tool for Continuous Investigation of the Evolution of the Casein Gel Microstructure after Renneting. Macromolecules, 41(6), 2071-2078. http://doi.org/10.1021/ma702246m

Le Feunteun, S., Ouethrani, M., \& Mariette, F. (2012). The rennet coagulation mechanisms of a concentrated casein suspension as observed by PFG-NMR diffusion measurements. Food Hydrocolloids, 27(2), 456-463. http://doi.org/10.1016/j.foodhyd.2011.09.008

Lorén, N., Nydén, M., \& Hermansson, A.-M. (2009a). Determination of local diffusion properties in heterogeneous biomaterials. Advances in Colloid and Interface Science, 150(1), 5-15. http://doi.org/10.1016/j.cis.2009.05.004

Lorén, N., Shtykova, L., Kidman, S., Jarvoll, P., Nydén, M., \& Hermansson, A.-M. (2009b). Dendrimer diffusion in kappa-carrageenan gel structures. Biomacromolecules, 10(2), 275-284. http://doi.org/10.1021/bm801013x

Mariette, F., Topgaard, D., Jönsson, B., \& Söderman, O. (2002). 1H NMR diffusometry study of water in casein dispersions and gels. Journal of Agricultural and Food Chemistry, 50(15), 4295-4302. http://doi.org/10.1021/jf0115948

Masaro, L., \& Zhu, X. (1999). Physical models of diffusion for polymer solutions, gels and solids. Progress in Polymer Science, 24(5), 731-775. http://doi.org/10.1016/S0079-6700(99)00016-7

Mason, T. G., \& Weitz, D. A. (1995). Linear viscoelasticity of colloidal hard sphere suspensions near the glass transition. Physical Review Letters, 75(14), 2770-2773. http://doi.org/10.1103/PhysRevLett.75.2770

Moschakis, T. (2013). Microrheology and particle tracking in food gels and emulsions. Current Opinion in Colloid \& Interface Science, 18(4), 311-323. http://doi.org/10.1016/j.cocis.2013.04.011

Mun, E. A., Hannell, C., Rogers, S. E., Hole, P., Williams, A. C., \& Khutoryanskiy, V. V. (2014). On the Role of Specific Interactions in the Diffusion of Nanoparticles in Aqueous Polymer Solutions. Langmuir, 30(1), 308-317. http://doi.org/10.1021/la4029035

Netz, P. A., \& Dorfmüller, T. (1995). Computer simulation studies of anomalous diffusion in gels: Structural properties and probe-size dependence. The Journal of Chemical Physics, 103(20), 9074. http://doi.org/10.1063/1.470018

Nydén, M., \& Söderman, O. (1998). An NMR Self-Diffusion Investigation of Aggregation Phenomena in Solutions of Ethyl(hydroxyethyl)cellulose. Macromolecules, 31(15), 4990-5002. http://doi.org/10.1021/ma971472

Ogston, A. G. (1958). The spaces in a uniform random suspension of fibres. Transactions of the Faraday Society, 54(0), 1754-1757. http://doi.org/10.1039/tf9585401754

Olmsted, S. S., Padgett, J. L., Yudin, A. I., Whaley, K. J., Moench, T. R., \& Cone, R. A. (2001). Diffusion of Macromolecules and Virus-Like Particles in Human Cervical Mucus. Biophysical Journal, 81(4), 1930- 
1937. http://doi.org/10.1016/S0006-3495(01)75844-4

Perry, P. A., Fitzgerald, M. A., \& Gilbert, R. G. (2006). Fluorescence Recovery after Photobleaching as a Probe of Diffusion in Starch Systems. Biomacromolecules, 7(2), 521-530. http://doi.org/10.1021/bm0507711

Petit, J. M., Roux, B., Zhu, X. X., \& Macdonald, P. M. (1996). A New Physical Model for the Diffusion of Solvents and Solute Probes in Polymer Solutions. Macromolecules, 29(18), 6031-6036. http://doi.org/10.1021/ma951159c

Petrášek, Z., Ries, J., \& Schwille, P. (2010). Scanning FCS for the characterization of protein dynamics in live cells. Methods in Enzymology. http:// doi.org/10.1016/S0076-6879(10)72005-X

Rathgeber, S., Beauvisage, H.-J., Chevreau, H., Willenbacher, N., \& Oelschlaeger, C. (2009). Microrheology with Fluorescence Correlation Spectroscopy. Langmuir, 25(11), 6368-6376. http://doi.org/10.1021/la804170k

Rubinstein, M., \& Colby, R. H. (2003). Polymer Physics. OUP Oxford.

Salami, S., Rondeau-Mouro, C., Barhoum, M., van Duynhoven, J., \& Mariette, F. (2014). Translational and rotational diffusion of flexible PEG and rigid dendrimer probes in sodium caseinate dispersions and acid gels. Biopolymers, 101(9), 959-965. http://doi.org/10.1002/bip.22492

Salami, S., Rondeau-Mouro, C., van Duynhoven, J., \& Mariette, F. (2013a). PFG-NMR self-diffusion in casein dispersions: Effects of probe size and protein aggregate size. Food Hydrocolloids, 31(2), 248-255. http://doi.org/10.1016/j.foodhyd.2012.10.020

Salami, S., Rondeau-Mouro, C., van Duynhoven, J., \& Mariette, F. (2013b). Probe Mobility in Native Phosphocaseinate Suspensions and in a Concentrated Rennet Gel: Effects of Probe Flexibility and Size. Journal of Agricultural and Food Chemistry, 61(24), 5870-5879. http://doi.org/10.1021/jf304949c

Sanders, L. M., \& Hendren, R. W. (2006). Protein Delivery. (L. M. Sanders \& R. W. Hendren, Eds.) (Vol. 10). Boston, MA: Springer Science \& Business Media. http://doi.org/10.1007/b112382

Schuster, B. S., Suk, J. S., Woodworth, G. F., \& Hanes, J. (2013). Nanoparticle diffusion in respiratory mucus from humans without lung disease. Biomaterials, 34(13), 3439-3446. http://doi.org/10.1016/j.biomaterials.2013.01.064

Schuster, E., Hermansson, A.-M., Öhgren, C., Rudemo, M., \& Lorén, N. (2014). Interactions and diffusion in fine-stranded $\beta$-lactoglobulin gels determined via FRAP and binding. Biophysical Journal, 106(1), 253262. http://doi.org/10.1016/j.bpj.2013.11.2959

Silva, J. V. C., Peixoto, P. D. S., Lortal, S., \& Floury, J. (2013). Transport phenomena in a model cheese: The influence of the charge and shape of solutes on diffusion. Journal of Dairy Science. http://doi.org/10.3168/jds.2013-6552

Speedy, R. J. (1987). Diffusion in the hard sphere fluid. Molecular Physics, 62(2), 509-515. http://doi.org/10.1080/00268978700102371

Stylianopoulos, T., Diop-Frimpong, B., Munn, L. L., \& Jain, R. K. (2010). Diffusion Anisotropy in Collagen Gels and Tumors: The Effect of Fiber Network Orientation. Biophysical Journal, 99(10), 3119-3128. http://doi.org/10.1016/j.bpj.2010.08.065

Takahashi, K., Kato, H., \& Kinugasa, S. (2011). Development of a Standard Method for Nanoparticle Sizing by Using the Angular Dependence of Dynamic Light Scattering. Analytical Sciences, 27(7), 751-756. http://doi.org/10.2116/analsci.27.751

Tsai, D. S., \& Strieder, W. (1986). Effective Conductivities of Random Fiber Beds. Chemical Engineering Communications, 40(1-6), 207-218. http://doi.org/10.1080/00986448608911698

Valentine, M., Kaplan, P., Thota, D., Crocker, J., Gisler, T., Prud'homme, R., et al. (2001). Investigating the microenvironments of inhomogeneous soft materials with multiple particle tracking. Physical Review E, 64(6), 061506. http://doi.org/10.1103/PhysRevE.64.061506

Wang, B., Kuo, J., Bae, S. C., \& Granick, S. (2012). When Brownian diffusion is not Gaussian. Nature Materials, 11(6), 481-485. http://doi.org/10.1038/nmat3308 
Wassén, S., Bordes, R., Gebäck, T., Bernin, D., Schuster, E., Lorén, N., \& Hermansson, A.-M. (2014). Probe diffusion in phase-separated bicontinuous biopolymer gels. Soft Matter, 10(41), 8276-8287. http://doi.org/10.1039/c4sm01513d

Weng, L., Liang, S., Zhang, L., Zhang, X., \& Xu, J. (2005). Transport of Glucose and Poly(ethylene glycol)s in Agarose Gels Studied by the Refractive Index Method. Macromolecules, 38(12), 5236-5242. http://doi.org/10.1021/ma047337w

Wong, I. Y., Gardel, M. L., Reichman, D. R., Weeks, E. R., Valentine, M. T., Bausch, A. R., \& Weitz, D. A. (2004). Anomalous diffusion probes microstructure dynamics of entangled F-actin networks. Physical Review Letters, 92(17), 178101. http://doi.org/10.1103/PhysRevLett.92.178101

Zareh, S. K., DeSantis, M. C., Kessler, J. M., Li, J.-L., \& Wang, Y. M. (2012). Single-Image Diffusion Coefficient Measurements of Proteins in Free Solution. Biophysical Journal, 102(7), 1685-1691. http://doi.org/10.1016/j.bpj.2012.02.030 



\title{
NMR nanoparticle diffusometry in hydrogels: Enhancing sensitivity and selectivity
}

\begin{abstract}
From the diffusional behavior of nanoparticles in heterogeneous hydrogels, quantitative information about sub-micron structural features of the polymer matrix can be derived. Pulsedgradient spin-echo NMR is often the method of choice, because it measures diffusion of the whole ensemble of nanoparticles. However, in ${ }^{1} \mathrm{H}$ diffusion-ordered spectroscopy (DOSY), low-intensity nanoparticle signals have to be separated from a highly protonated background. To circumvent this, we prepared ${ }^{19} \mathrm{~F}$ labeled, PEGylated, water-soluble dendritic nanoparticles with a ${ }^{19} \mathrm{~F}$ loading of $\sim 7 \mathrm{wt} \%$ to enable background free ${ }^{19} \mathrm{~F}$ DOSY experiments. ${ }^{19} \mathrm{~F}$ nanoparticle diffusometry was benchmarked against ${ }^{1} \mathrm{H}$ diffusion- $T_{2}$ correlation spectroscopy (DRCOSY), which has a stronger signal separation potential than the commonly used ${ }^{1} \mathrm{H}$ DOSY experiment. We used bootstrap data resampling to estimate confidence intervals and stabilize 2D-Laplace inversion of DRCOSY data with high noise levels and artifacts, allowing quantitative diffusometry even at low magnetic field strengths $(30 \mathrm{MHz})$. The employed methods offer significant advantages in terms of sensitivity and selectivity.
\end{abstract}
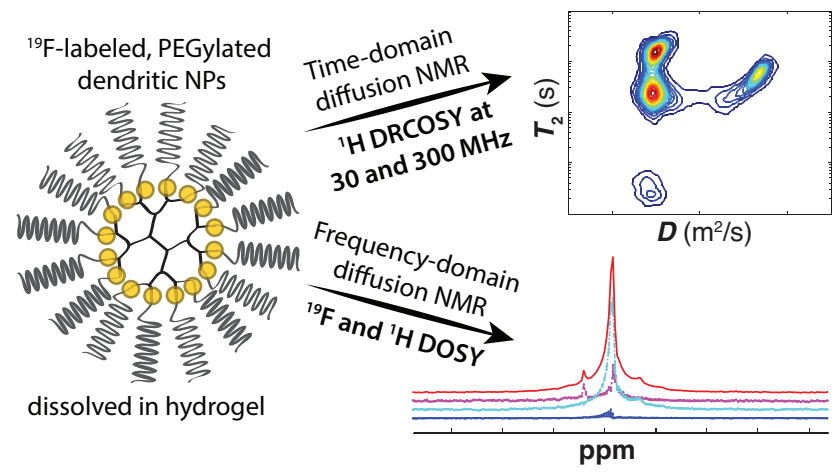

Daan W. de Kort, John P.M. van Duynhoven, Freek J.M. Hoeben, Henk M. Janssen, and Henk Van As, Analytical Chemistry 2014, 86 (18), pp 9229-9235. DOI: 10.1021/ac502211q 


\subsection{Introduction}

Biopolymer-based hydrogels are widely applied in technological applications, such as barrier and separation materials, controlled release media, and in foods and cosmetics. They derive many of their technologically relevant physical parameters from their sub-micron structural features. Traditionally, these features have been studied by (electron) microscopic techniques, but these methods are invasive and require careful image analysis in order to quantify network parameters. An increasingly popular method for the non-invasive characterization of heterogeneous hydrogels is through the observation of hindered diffusion of embedded nanoparticles with diameters in the 1-100 nanometer range. Physical models are available to describe hindered diffusion of nanoparticles in polymer gels (Masaro \& Zhu, 1999) and solutions (Cai, Panyukov, \& Rubinstein, 2011) in terms of structural length scales and dynamics. These physical models allow quantitative network descriptors to be derived from experimental nanoparticle diffusion data. Quantitative nanoparticle diffusometry has been convincingly demonstrated in various biopolymer model systems, including alginate (Bernin et al., 2011), א-carrageenan (Lorén et al., 2009), casein (Salami, Rondeau-Mouro, van Duynhoven, \& Mariette, 2013b; 2013a), gelatin (Hagman, Lorén, \& Hermansson, 2010), whey (Colsenet, Söderman, \& Mariette, 2006) and collagen (Stylianopoulos, Diop-Frimpong, Munn, \& Jain, 2010) gels.

Optical methods to measure nanoparticle self-diffusion in complex materials, including fluorescence recovery after photobleaching (Axelrod, Koppel, Schlessinger, Elson, \& Webb, 1976; Braeckmans, Peeters, Sanders, De Smedt, \& DeMeester, 2003) (FRAP) and fluorescence correlation spectroscopy (Rathgeber, Beauvisage, Chevreau, Willenbacher, \& Oelschlaeger, 2009) (FCS), require fluorescent labeling of particles and optical transparency of the material. Particularly in heterogeneous biopolymer gels, optical transparency issues limit the use of these methods.

For diffusion measurements by NMR, we need nanoparticles with NMR-observable nuclei in molecular segments with liquid-like reorientation dynamics. Optical transparency of the material is, however, not required. For this reason, NMR is as a robust method for nanoparticle diffusometry in biopolymer gels.

Dendrimers are ideal diffusional probes, because they are essentially monodisperse due to controlled, stepwise size increments during synthesis (Cheng, Prud'homme, \& Thomas, 2002). Nanoparticles are observed from their ${ }^{1} \mathrm{H}$ signals, but since they are typically dosed at low levels $(\sim 0.1 \mathrm{wt} \%)$ in order not to perturb submicron structure, ${ }^{1} \mathrm{H}$ diffusometry suffers from low selectivity for particles against a highly protonated background of water and polymer matrix. Although water and matrix signals can, for a large part, be suppressed through spectral editing (Liu, Nicholson, \& Lindon, 1996), any remaining background signal can cause difficulties in the interpretation of nanoparticle diffusometry experiments.

One route to overcome the selectivity issues of ${ }^{1} \mathrm{H}$ NMR nanoparticle diffusometry is to label them with an NMR observable isotope that is otherwise not present in the hydrogel matrix. Due to 
its high gyromagnetic ratio, labeling nanoparticles with ${ }^{19} \mathrm{~F}$ is attractive and will allow for sensitive and background free detection.

A second route to achieve optimal separation of nanoparticle and background signals is to use diffusion- $T_{2}$ relaxation correlation spectroscopy (DRCOSY) instead of the more commonly used diffusion-ordered spectroscopy (DOSY). $T_{2}$, the spin-spin relaxation time, is the time constant that describes how fast transverse magnetization decays to zero. The building blocks of these techniques and required data transformations are summarized in Table 3.1.

In DOSY, free-induction decays (FID) are Fourier transformed to obtain NMR spectra and the pulsed gradient spin echo (PGSE) dimension is fitted with attenuation exponentials using appropriate procedures, such as SplMod (van Resandt, Vogel, \& Provencher, 1982), CONTIN (Provencher, 1982), or multivariate methods (Huo, Wehrens, van Duynhoven, \& Buydens, 2003; Nilsson \& Morris, 2007), to obtain associated diffusion coefficients. Here, we will be using SplMod because of its ability to handle exponential sampling of the gradient axis and perform a "coupled fit" of all spectral points, resulting in a spectral decomposition.

\begin{tabular}{llll}
\hline & $\boldsymbol{F}_{\mathbf{2}}$ & $\begin{array}{l}\boldsymbol{F}_{\mathbf{1}} \\
\text { acquisition }\end{array}$ & $\begin{array}{l}\text { transformations/ } \\
\text { fitting procedures }\end{array}$ \\
\hline \multirow{2}{*}{ DOSY } & PGSE & FID & $\begin{array}{l}\boldsymbol{F}_{\mathbf{1}} \text { : Fourier transform } \\
\boldsymbol{F}_{2} \text { : discrete exponential fit by } \\
\text { SplMod (van Resandt et al., 1982) }\end{array}$ \\
\hline \multirow{2}{*}{ DRCOSY } & \multirow{2}{*}{ PGSE } & $\begin{array}{l}\text { time domain } \\
\text { CPMG }\end{array}$ & $\boldsymbol{F}_{2}, \boldsymbol{F}_{\mathbf{1}}$ : 2D Laplace inversion by \\
& & FLI (Song et al., 2002) \\
\hline
\end{tabular}

Table 3.1: Building blocks of diffusion-ordered spectroscopy (DOSY) and diffusion-relaxation correlation spectroscopy (DRCOSY) experiments with required data transformations. The PGSE block is based on the stimulated echo: $90^{\circ}-G-90^{\circ}-\tau_{z}-90^{\circ}-G$, where diffusion observation time equals the time between gradient pulses G. DOSY is composed of a pulsed-gradient spin-echo (PGSE) type diffusion block in the $F_{2}$ domain followed by acquisition of the free induction decay (FID) in $F_{1}$. FIDs are Fourier transformed to obtain NMR spectra. Spectra are then fitted with a discrete sum of attenuation exponentials using SplMod (van Resandt et al., 1982). DRCOSY is composed of a PGSE type diffusion experiment followed by time-domain Carr-Purcell-Meiboom-Gill (CPMG, $\left[180^{\circ}-\tau_{e}\right]_{n}$, where $\tau_{e}$ equals inter-echo time) acquisition. Two-dimensional Laplace inversion (2D-ILT) using a fast algorithm (Song et al., 2002) yields the diffusion- $T_{2}$ correlation map. 


\begin{tabular}{lll}
\hline & high field & low field \\
& $7.0 \mathrm{~T}$ & $0.7 \mathrm{~T}$ \\
\hline DRCOSY & ${ }^{1} \mathrm{H}$ & ${ }^{1} \mathrm{H}$ \\
\hline \multirow{2}{*}{ DOSY } & ${ }^{1} \mathrm{H}$ & $\mathrm{n} / \mathrm{a}$
\end{tabular}

Table 3.2: Diffusometry experiments in this work.

DOSY experiments typically cannot be performed at low field strength, because of poor magnet homogeneity in combination with low chemical shift separation of signals. ${ }^{19} \mathrm{~F}$ experiments were not performed at low field strength, because of low sensitivity due to the relatively small ${ }^{19} \mathrm{~F}$ payload per particle and low particle concentrations.

DRCOSY is a correlation experiment that combines the PGSE and Carr-Purcell-Meiboom-Gill (CPMG) experiments. Acquisition of the NMR signal takes place in the time domain (i.e., all echo intensities are recorded during the CPMG experiment). Time domain acquisition allows the total acquisition time of DRCOSY to equal that of DOSY. 2D Laplace inversion of the data yields a diffusion- $T_{2}$ correlation map.

Here, we compare DOSY and DRCOSY ${ }^{19} \mathrm{~F}$ and ${ }^{1} \mathrm{H}$ NMR diffusometry of ${ }^{19} \mathrm{~F}$-labeled, PEGylated dendrimer nanoparticles (SyMO-Chem B.V.) in a $\kappa$-carrageenan gel model system. We performed ${ }^{19} \mathrm{~F}$ and ${ }^{1} \mathrm{H}$ DOSY at $7 \mathrm{~T}$ field strength and ${ }^{1} \mathrm{H}$ DROSY at $7 \mathrm{~T}$ and $0.7 \mathrm{~T}$ field strength (Table 3.2). ${ }^{19} \mathrm{~F}$ DRCOSY at high field was not performed, since it does not offer advantages over ${ }^{19} \mathrm{~F}$ DOSY in which only a single chemical shift is present. DOSY is then preferred over DRCOSY because of the stability of FT over Laplace inversion.

$\kappa$-Carrageenan gels were chosen as model system because of their sub-micron structural heterogeneity. Lorén et al. observed bimodal diffusion of nanoparticles in $\kappa$-carrageenan gels, which could be a result of the presence of micro-domains in which nanoparticles have truly different diffusion coefficients (Lorén et al., 2009). We will assess the performance of both DOSY and DRCOSY for resolving bimodality of diffusion.

\subsection{Materials and Methods}

\subsubsection{Design and relaxometry of labeled nanoparticles}

Design. Poly (propylene imine) (PPI) based dendrimers were used as a chassis for constructing ${ }^{19} \mathrm{~F}$ labeled nanoparticles (SyMO-Chem B.V.). Three generations (G1, G3 and G5) of PPI dendrimers were functionalized with $\mathrm{C}^{19} \mathrm{~F}_{3}$ and coated with PEG (Figure 3.1). PEGylation solubilizes the particles and prevents interactions between the particles and the biopolymer matrix. In order to avoid compromised solubility of the dendrimers, the hydrophobic $\mathrm{C}^{19} \mathrm{~F}_{3}$ functional groups were introduced at the interface between dendritic core and PEG corona. 


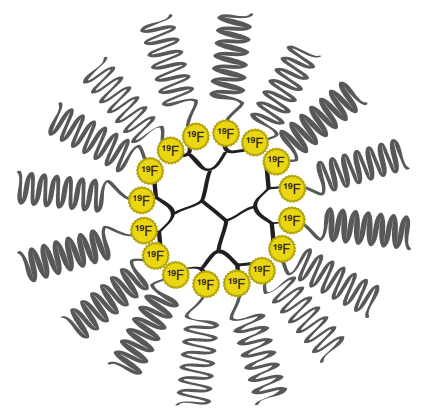

Figure 3.1: Design of $\mathrm{C}^{19} \mathrm{~F}_{3}$-functionalized nanoparticles, based on polypropylene-imine dendrimer cores (in black) of generations G1, G3 and G5, and a polyethylene glycol (PEG) corona (in gray), which prevents sticking of particles to the biopolymer network. ${ }^{19} \mathrm{~F}$ labels (in yellow) have been introduced at the interface between the dendritic core and PEG corona.

NMR relaxometry. ${ }^{19} \mathrm{~F}$-labeled, PEGylated dendritic nanoparticles were characterized by ${ }^{19} \mathrm{~F}$ and ${ }^{1} \mathrm{H}$ relaxometry. Experiments were performed on a Bruker Avance II spectrometer, equipped with a Bruker diff25 probe, at $7.0 \mathrm{~T}\left(300 \mathrm{MHz}\right.$ for ${ }^{1} \mathrm{H}$ and $282 \mathrm{MHz}$ for $\left.{ }^{19} \mathrm{~F}\right)$, in water at $298 \mathrm{~K}$. The probe was equipped with a 10-mm RF insert that could be tuned to both ${ }^{1} \mathrm{H}$ and ${ }^{19} \mathrm{~F}$. Sample volume was chosen as to not exceed the NMR coil volume. Longitudinal relaxation $\left(T_{1}\right)$ was measured by an inversion-recovery experiment $\left(180^{\circ}-\tau_{i r}-90^{\circ}\right)$. Transverse relaxation $\left(T_{2}\right)$ was measured by a frequency-domain CPMG experiment with an inter-echo time of $1 \mathrm{~ms}$. In the ${ }^{1} \mathrm{H}$ case, PGSE diffusion editing was used to suppress the water signal.

\subsubsection{Preparation of $\kappa$-carrageenan gels}

$\kappa$-Carrageenan gels were prepared by dissolution of $\kappa$-carrageenan powder (Sigma-Aldrich), $\mathrm{NaCl}$ and $\mathrm{KCl}$ salts and nanoparticles in water, as described by Lorén et al. (Lorén et al., 2009) $\kappa$ Carrageenan weight fraction was varied between $1 \%$ and $5 \%$ in five steps. In all experiments, nanoparticles were dosed at $0.1 \mathrm{wt} \%$; $\mathrm{NaCl}$ concentration was kept at $200 \mathrm{mM}$ and $\mathrm{KCl}$ concentration at $20 \mathrm{mM}$. Molten gels were transferred into NMR-tubes, where the filling height was chosen not to exceed the linear part of the magnetic field gradient. Gels were allowed to stabilize for 24 hours before measurements were performed.

\subsubsection{NMR diffusometry}

PGSE experiments were carried out by stepwise variation of the gradient pulse amplitude, while keeping the diffusion-observation time and gradient pulse width constant. The attenuation of signal intensity as a function of the experimental parameters is described by the Stejskal-Tanner equation $\frac{I}{I_{0}}=e^{-q^{2}(\Delta-\delta / 3) D}$, where $\frac{I}{I_{0}}$ is the signal attenuation, $\Delta$ the effective diffusion time (s) and $D$ the diffusion coefficient $\left(\mathrm{m}^{2} \mathrm{~s}^{-1}\right)$ and $q=\gamma \delta g$, where $\gamma$ is the gyromagnetic ratio of the observed nucleus ( $\left.\mathrm{rad} \mathrm{T}^{-1} \mathrm{~s}^{-1}\right), g$ is the magnetic field gradient amplitude $\left(\mathrm{T} \mathrm{m}^{-1}\right)$ and $\delta$ is the effective gradient pulse width (s), where $\delta \ll \Delta$ (narrow gradient pulse approximation) (Stejskal \& Tanner, 1965). Bimodality of diffusion in heterogeneous gels can manifest itself through bi- 
exponential attenuation of the NMR signal with increasing gradient strength (Lorén et al., 2009). This means that a curve of signal intensity vs. $q$ can be fitted with a discrete sum of attenuation exponentials $\frac{I}{I_{0}}=\sum_{i} A_{i} e^{-q^{2}(\Delta-\delta / 3) D_{i}}$, where $A_{\mathrm{i}}$ is the amplitude and $D_{i}$ the diffusion coefficient of component $i$.

Apparatus. High-field ${ }^{19} \mathrm{~F}$ and ${ }^{1} \mathrm{H}$ DOSY and ${ }^{1} \mathrm{H}$ DRCOSY NMR experiments were performed on a Bruker Avance II spectrometer at 7.0 T equipped with a Bruker diff25 gradient probe as described above. This probe generates a maximum field gradient strength of $9.6 \mathrm{~T} \mathrm{~m}^{-1}$. Sample temperature was kept at $293 \mathrm{~K}$, regulated indirectly through the gradient-coil cooling system to prevent any temperature gradients across the sample. Low field ${ }^{1} \mathrm{H}$ DRCOSY experiments were performed on a Maran Ultra spectrometer equipped with a $0.7 \mathrm{~T}$ permanent magnet $\left(30 \mathrm{MHz}\right.$ for $\left.{ }^{1} \mathrm{H}\right)$, equipped with a magnetic field gradient coil that generates a maximum gradient strength of $1.2 \mathrm{~T} \mathrm{~m}^{-1}$. Low field experiments were performed at room temperature.

${ }^{1} \mathrm{H}(300 \mathrm{MHz})$ and ${ }^{19} \mathrm{~F}(282 \mathrm{MHz})$ DOSY. DOSY experiments were performed on G1, G3 and G5 dendrimers in water and carrageenan gels. A stimulated echo-based PGSE diffusion experiment was used in combination with unipolar, trapezoid-shaped gradient pulses. In all DOSY experiments, an effective diffusion time $\Delta$ of $200 \mathrm{~ms}$ was used, and an effective gradient pulse width $\delta$ of $1.5 \mathrm{~ms}$. The gradient strength was varied in a logarithmic manner between $0.5-4.5 \mathrm{~T} \mathrm{~m}^{-}$ ${ }^{1}$ for ${ }^{1} \mathrm{H}$ and $0.03-4.5 \mathrm{~T} \mathrm{~m}^{-1}$ for ${ }^{19} \mathrm{~F}$ in 64 steps. A stimulated-echo-based PGSE experiment requires nonzero initial gradient amplitude. However, the initial gradient was stronger in the ${ }^{1} \mathrm{H}$ case than in the ${ }^{19} \mathrm{~F}$ case in order to effectively suppress the intense ${ }^{1} \mathrm{H}$ signal of water, whose diffusion coefficient is over 10 times higher than that of dendrimer nanoparticles. For ${ }^{1} \mathrm{H}$ NMR measurements, the NMR signal was averaged 16 times and for ${ }^{19} \mathrm{~F}$ NMR 64 times. Experimental repetition time was set at 3 times $T_{1}$ of PEG and ${ }^{19} \mathrm{~F}$ labels, respectively.

High field ${ }^{1} \mathrm{H}(300 \mathrm{MHz})$ DRCOSY. High field DRCOSY experiments were performed on G1, G3 and G5 dendrimers in water and carrageenan gels. Experiments were based on a stimulated echobased PGSE diffusion experiment with unipolar, trapezoid-shaped gradient pulses, followed by time-domain CPMG acquisition. Experimental parameters for the PGSE block were the same as those used in ${ }^{1} \mathrm{H}$ DOSY. For CPMG, an inter-echo time of $1 \mathrm{~ms}$ was used and 2048 echo intensities were recorded. The NMR signal was averaged 16 times. Experimental repetition time was set at 3 times $T_{1}$ of PEG.

Low field ${ }^{1} \mathrm{H}(30 \mathrm{MHz})$ DRCOSY. Low field DRCOSY experiments were performed on G3 dendrimers in water and carrageenan gels. The experimental parameters for the PGSE and CPMG blocks were identical to those used for high field DRCOSY, except that gradient strength was 
varied in a logarithmic manner between $0.5-1.2 \mathrm{~T} \mathrm{~m}^{-1}$ in 64 steps. The NMR signal was averaged 256 times. Experimental repetition time was set at 3 times $T_{1}$ of PEG.

Data analysis. We used bootstrap data resampling (Efron, 1979) as a simple but effective tool to estimate confidence intervals in PGSE and CPMG data. Furthermore we found that bootstrap resampling is an effective method to stabilize 2D Laplace inversion of DRCOSY data subject to high noise levels and artifacts that would otherwise lead to "spurious" peaks in the $D-T_{2}$ correlation maps.

Bootstrap resampling was implemented as transformation of subsequent random sub-selections of data points, and summation of the resulting intensity spectra or correlation maps. Bootstrapping is an adequate statistical method, because the distribution of statistics (diffusion coefficient, $T_{2}$ ) is not known (deviations from the bimodal model might occur in case of diffusive exchange), and the sample size is low, particularly in the PGSE dimension ( $\sim 10^{1}$ data points). Previously, random addition of noise has been used as an analogous method to estimate errors in PGSE data (Alper \& Gelb, 1990). Bootstrap resampling, however, does not manipulate data points and offers the additional benefit of evaluating model robustness, besides evaluating the effects of noise.

Analysis of DOSY experiments. NMR spectra were obtained through Fourier transformation of FIDs and subsequent phasing using standard procedures. In the PGSE dimension 1000 bootstrap samples of 64 spectra were prepared. These 1000 bootstrap samples were successively fitted with 1-3 attenuation exponentials through SplMod (van Resandt et al., 1982; Vogel, 1988). For analyzing ${ }^{1} \mathrm{H}$ NMR diffusometric data, SplMod was used in coupled mode in order to achieve spectral decomposition. The nonzero initial gradient amplitude was set to $q=0$ in order to suppress the water signal in the resulting intensity spectra. This procedure only changes the point at which the attenuation curve intercepts the amplitude axis and does not influence the estimated diffusion coefficients, which follow from the attenuation rate as a function of $q$. In the ${ }^{19} \mathrm{~F}$ case, the single $\mathrm{C}^{19} \mathrm{~F}_{3}$ resonance was integrated and fitted by SplMod (not in coupled mode, because spectral decomposition is not required). Basic bootstrapped confidence limits were obtained by finding the median and one-standard-deviation quantiles of the relevant parameters (Davison \& Hinkley, 1997).

Analysis of DRCOSY experiments. A zeroth-order phase correction was applied to the CPMG echo train. Subsequently, the data were bootstrapped and analyzed through Fast 2D Laplace Inversion (FLI) (Song et al., 2002). As in ${ }^{1} \mathrm{H}$ DOSY, the intense water signal was suppressed by a strong initial gradient. The PGSE axis, which starts at a nonzero value, was again shifted to zero initial gradient amplitude $(q=0)$, in order to suppress the intense water signal. Before 2D Laplace inversion, 250 bootstrap samples were prepared of both the PGSE and CPMG dimensions and combined into 250 two-dimensional reference matrices. Data points selected through these 
matrices were subsequently inverted by FLI. For all bootstrap fits, the regularization ("smoothing") parameter was fixed at a low value. Because FLI output is the intensity map backpredicted to $(t=0, q=0)$, resulting maps could simply be summed to obtain an averaged map, in which robust elements would survive and spurious peaks would disappear. Unlike in the DOSY case, bootstrapped parameter distributions could not be obtained directly; Instead, we determined the signal width (FWHM) in the bootstrapped intensity map in order to estimate the error.

\subsection{Results and Discussion}

\subsubsection{Characterization of ${ }^{19} \mathrm{~F}$ labeled dendrimers}

By attaching ${ }^{19} \mathrm{~F}$ labels in-between dendritic cores and PEG coronas, we introduced ${ }^{19} \mathrm{~F}$ payloads of $\sim 7 \mathrm{wt} \%$ without affecting the water solubility of the dendrimer constructs. In the current design, the ratio between ${ }^{19} \mathrm{~F}$ nuclei and PEG ${ }^{1} \mathrm{H}$ nuclei is $1: 15$. Higher ${ }^{19} \mathrm{~F}$ payloads should be attainable with the used PPI architectures via a similar design. ${ }^{19} \mathrm{~F}$ NMR spectra of three generations of $\mathrm{C}^{19} \mathrm{~F}_{3^{-}}$ functionalized dendritic nanoparticles showed a single resonance line $(-64 \mathrm{ppm})$. The ${ }^{1} \mathrm{H}$ spectrum showed a prominent signal from the PEG corona $(3.7 \mathrm{ppm})$ and several broad dendritic signals. Relaxation parameters of ${ }^{19} \mathrm{~F}$ labels and PEG groups at 7.0 T are presented in Table S6.1. Longitudinal $\left(T_{1}\right)$ and transverse $\left(T_{2}\right)$ relaxation times of polyethylene glycol (PEG) corona and ${ }^{19} \mathrm{~F}$ labels at 7.0 T field strength allow for diffusion-observation times of order $10^{1}-10^{2} \mathrm{~ms}$. Although it can be predicted that the use of spin-echo-based PGSE would result in a higher S/N than the use of a stimulated echo, we chose to use stimulated-echo based PGSE, because $T_{2}$ might shift to lower values when nanoparticles are embedded in dense polymer gels. Hydrodynamic diameters of nanoparticles were estimated from their diffusion coefficients in water at $298 \mathrm{~K}$, as measured by DOSY, through the Stokes-Einstein equation $3 \pi \eta d_{\mathrm{h}} D=k_{b} T$ where $\eta$ is solvent dynamic viscosity $(1.002 \mathrm{mPa} s$ for water at $298 \mathrm{~K}), d_{\mathrm{h}}$ hydrodynamic particle diameter, $D$ diffusion coefficient, $k_{b}$ Boltzmann constant and $T$ temperature. Hydrodynamic particle diameters were found to be 3.4, 4.6 and $7.5 \mathrm{~nm}$ for G1, G3 and G5 dendrimers, respectively. Nanoparticles dissolved in water and stored at room temperature were stable over periods of months, as confirmed by invariant ${ }^{19} \mathrm{~F}$ and ${ }^{1} \mathrm{H}$ NMR spectra and self-diffusion coefficients.

\subsubsection{NMR diffusometry}

In heterogeneous systems, multi-modal diffusion will be observed only at time scales smaller than required for particles to exchange between different domains. At longer time scales, a single diffusion coefficient will be observed again (central limit theorem). In $\kappa$-carrageenan gels, varying the experimental diffusion-observation time did not change the apparent diffusion behavior. This implies that particle exchange is much slower than typical diffusion-observation times accessible with PGSE NMR. We consequently chose a diffusion observation time of $200 \mathrm{~ms}$, at which the narrow gradient pulse requirement is satisfied. 
${ }^{19} \mathrm{~F}(282 \mathrm{MHz})$ and ${ }^{1} \mathrm{H}(300 \mathrm{MHz})$ DOSY. Because pulsed-field gradient probeheads require smaller-than-usual sample volumes in view of gradient linearity requirements, it is difficult to obtain high magnetic field homogeneity within the sample. This leads to strong overlap of signals in the NMR spectrum. DOSY, which relies on acquisition of the NMR spectrum, did therefore not strongly separate nanoparticle and background signals adequately in the spectral domain. We fitted up to three attenuation exponentials to both ${ }^{1} \mathrm{H}$ and ${ }^{19} \mathrm{~F}$ DOSY spectra and the resulting diffusion coefficients and bootstrapped error intervals were calculated.

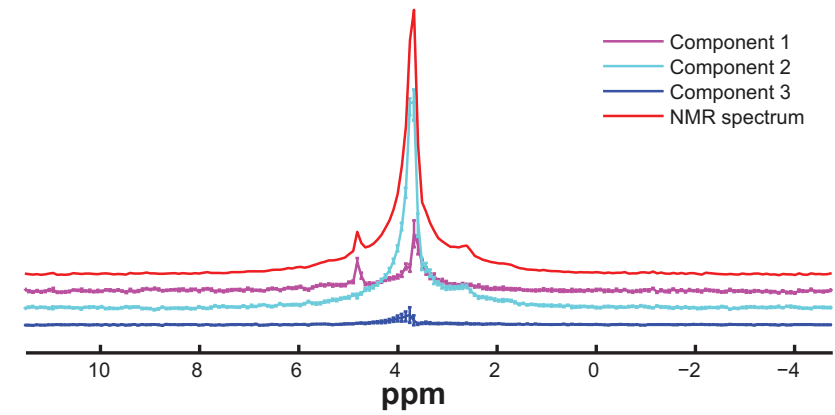

Figure 3.2: Representative result of ${ }^{1} \mathrm{H}$ DOSY three-component spectral decomposition by SplMod in coupled mode ( $5 \mathrm{wt} \%$ carrageenan containing $0.1 \mathrm{wt} \% \mathrm{G} 5$ dendrimers). Error bars represent 1 standard deviation.

A representative three-component spectral decomposition of the ${ }^{1} \mathrm{H}$ DOSY data is presented in Figure 3.2. It can be seen that all signals are broad and distorted, due to low magnetic field homogeneity. The first component, which has the largest diffusion coefficient $\left(\sim 10^{-10} \mathrm{~m}^{2} \mathrm{~s}^{-1}\right)$, corresponds to sucrose (with a contribution of non-suppressed water), an impurity in some of the commercially available $\kappa$-carrageenan batches (SI, section S3.3). The sucrose NMR spectrum includes a broad range of chemical shifts in the sugar region. The second component $\left(D \sim 10^{-11} \mathrm{~m}^{2}\right.$ $\mathrm{s}^{-1}$ ) corresponds to the PEG corona of the dendrimer particles. The diffusion coefficient of this component decreases as a function of $\kappa$-carrageenan concentration. From this effect, structural properties of the matrix can be calculated through physical models of diffusion in polymer gels (Masaro \& Zhu, 1999). The third component $\left(D \sim 10^{-13} \mathrm{~m}^{2} \mathrm{~s}^{-1}\right)$ can correspond to slow-diffusing particles, (immobile) $\kappa$-carrageenan matrix (Supporting Information, section S3.3), or both. The presence of a slow-diffusing particle fraction can either indicate the presence of micro-domains with different polymer densities in which particles have different diffusion coefficients, or that the polymer matrix acts as a confinement in which particles can become restricted in their diffusion. In ${ }^{1} \mathrm{H}$ DOSY, however, slow-diffusing particles and $\kappa$-carrageenan signal cannot be properly separated due to both their low intensities and low diffusion coefficients. Low diffusion 
coefficients are difficult to resolve due to limited PFG amplitudes. From ${ }^{1} \mathrm{H}$ DOSY alone, the existence of a slow-diffusing fraction can therefore not be unambiguously established.

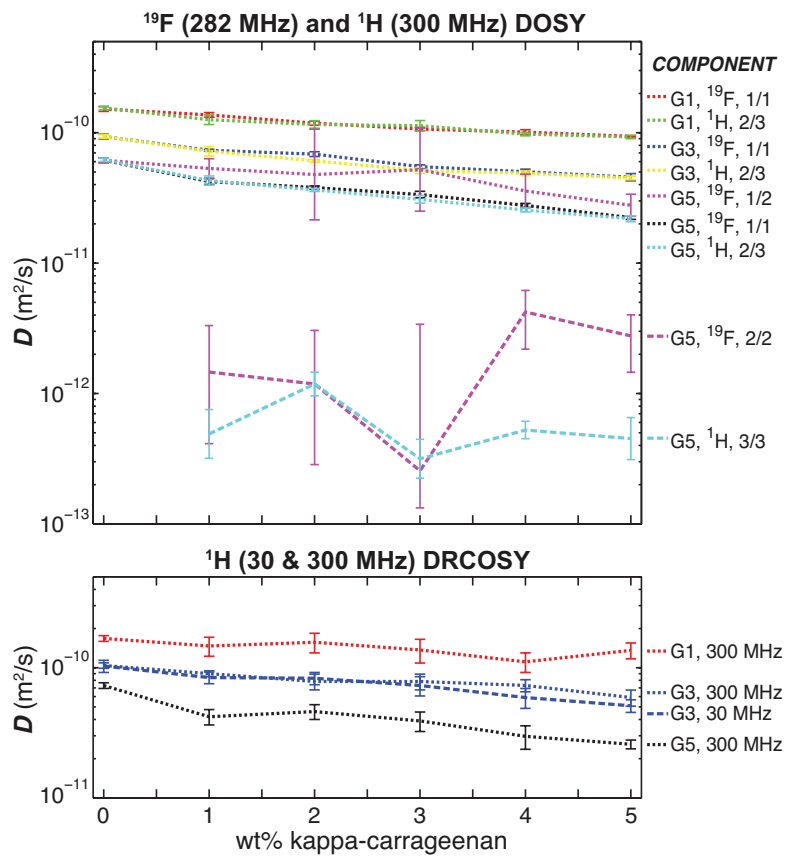

Figure 3.3: Diffusion of G1, G3 and G5 dendritic nanoparticles in $\kappa$-carrageenan. Upper panel: diffusion coefficients derived from SplMod fits of ${ }^{19} \mathrm{~F}$ DOSY and coupled SplMod fits of ${ }^{1} \mathrm{H}$ DOSY spectra. Error bars represent 1 standard deviation. ${ }^{19} \mathrm{~F}$ DOSY spectra were fitted with one component for G1 and G3 and G5, and two components for G5. ${ }^{1} \mathrm{H}$ DOSY spectra were fitted with three exponentials. For ${ }^{1} \mathrm{H}$, only nanoparticle-associated components are shown. Lower panel: Diffusion coefficients derived from ${ }^{1} \mathrm{H}$ DRCOSY at high and low field, fast diffusing fraction only. For DRCOSY, errors were estimated from correlation peak width in bootstrap-resampled diffusion- $T_{2}$ maps. 

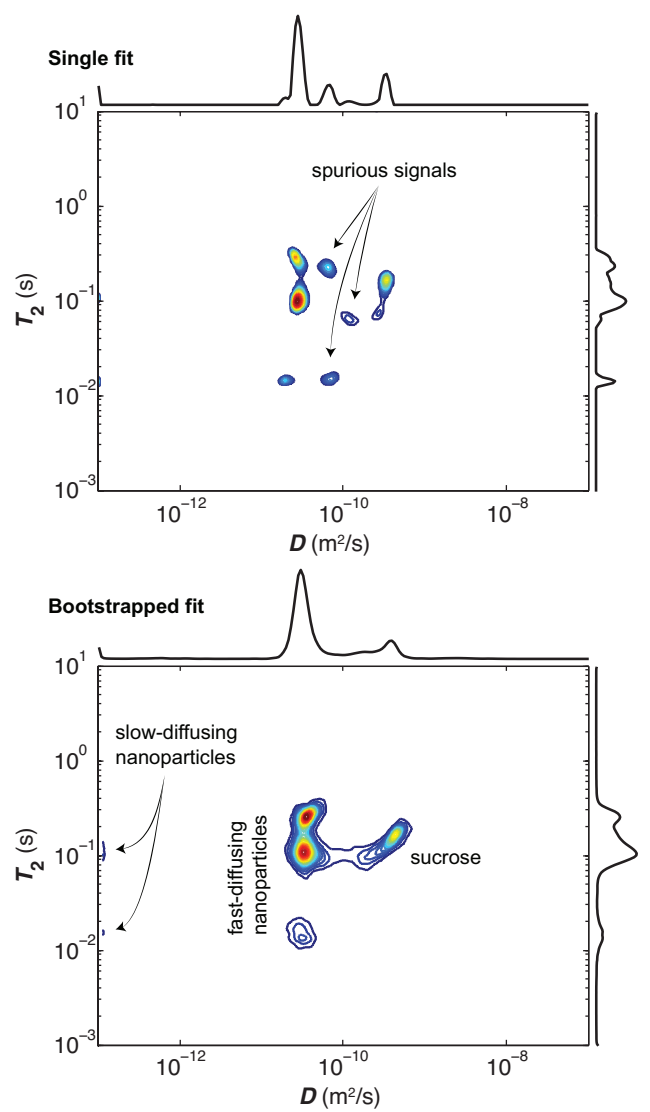

Figure 3.4: Representative ${ }^{1} \mathrm{H}$ diffusion- $T_{2}$ relaxation correlation maps of dendritic nanoparticles in $\kappa$ carrageenan gels ( $4 \mathrm{wt} \% \mathrm{k}$-carrageenan, G5 dendrimers). Single fit of raw data is shown in the upper panel, fit of bootstrap-resampled data in the lower panel.

In ${ }^{19} \mathrm{~F}$ DOSY, water, solutes and matrix signals are not present. We have compared one- and twocomponent models for ${ }^{19} \mathrm{~F}$ with the three-component model for ${ }^{1} \mathrm{H}$ DOSY. If a slow-diffusing particle fraction is indeed present, we expect to observe two components in ${ }^{19} \mathrm{~F}$ DOSY data. In Figure 3.3, upper panel, it can be seen that for all particles, close overlap of ${ }^{19} \mathrm{~F}$ data with the second component of ${ }^{1} \mathrm{H}$ DOSY is found with a one-component fit of ${ }^{19} \mathrm{~F}$ data. However, (only) for G5 particles, a two-component fit also gives satisfactory agreement with ${ }^{1} \mathrm{H}$ data. Furthermore, for G5 particles, fitting a second component leads to a relatively strong decrease of the standard deviation of the fit (SI, Figures S3.2c, S3.2g, S3.2h, S3.2i). We thus find a slow-diffusing particle fraction only for G5 particles. As was the case for ${ }^{1} \mathrm{H}$ DOSY, the slow-diffusing fraction is ill resolved due to its low intensity and diffusion coefficient. It can be seen in Figure 3.3, however, 
that the ${ }^{1} \mathrm{H}$ DOSY, third component and ${ }^{19} \mathrm{~F}$ DOSY, second component for G5 particles are in agreement within the same order of magnitude.

${ }^{1} \mathrm{H}$ DRCOSY at high $(300 \mathrm{MHz})$ and low $(30 \mathrm{MHz})$ field strengths. Since DRCOSY does not rely on separation of signals on the basis of chemical shift, there is no requirement for the magnetic field to be as homogeneous as for the DOSY case; During CPMG, successive spin echoes will refocus dephasing due to static field inhomogeneity. Since there is no need for spectral resolution, DRCOSY can also be used at low field strengths. At first sight, therefore, DRCOSY seems a more appropriate method for nanoparticle diffusometry. A drawback of DRCOSY, however, is the necessity to use $2 \mathrm{D}$ Laplace inversion to obtain the diffusion $-T_{2}$ relaxation correlation map. Laplace inversion is an ill-conditioned problem in that the signal matrix cannot be inverted robustly under the effects noise and signal artifacts, and a large number of solutions exist that fit the data within the experimental error. A small change in the signal, e.g. due to noise or artifacts, can lead to a large change in the solution. Generally the output is the simplest (most parsimonious) solution that is consistent with experimental data, generally with smoothness of the distribution as a constraint (Provencher, 1982). Standard regularization methods for 2D Laplace inversions such as Tikhonov regularization are used to impose this smoothness. This way, noisefree solutions are obtained, but the solution is generally over-smoothed (Berman, Levi, Parmet, Saunders, \& Wiesman, 2013).

Due to the low dosage levels of nanoparticles, noise and artifacts (e.g. small echo modulations due to spectrometer imperfections) become significant. Here, we use very little regularization and alternatively use bootstrap data resampling both as a tool to estimate confidence intervals and to average out the effects of noise and artifacts. We prefer bootstrapping to regularization because we found that regularization does not stabilize our correlation maps as consistently as bootstrapping. The cause of this must lie in the effects of signal artifacts rather than of noise. As opposed to noise, artifacts are not necessarily randomly distributed, which can lead to a consistent peak in $D-T_{2}$ correlation maps. Bootstrapping will remove peaks of such artifacts, at least if they cannot be described by an exponential function.

We have illustrated the effects of bootstrap resampling in Figure 3.4, where we compare diffusion$T_{2}$ correlation maps of raw and bootstrapped data. Bootstrapping leads to a stabilization of diffusion- $T_{2}$ correlation maps, as can be seen from the fact that signals in bootstrapped maps persist in all samples (cf. SI, section S3.1). When comparing the two maps, it can be seen that bootstrap resampling removes some spurious signals and broadens persistent correlation peaks. The additional broadening introduced by bootstrap data resampling can be interpreted as a measure for the confidence interval of diffusion coefficients and $T_{2}$ relaxation times.

In general, nanoparticles give rise to three correlation peaks with a common diffusion coefficient. As determined by a CPMG pulse train with subsequent acquisition of the NMR spectrum, the component with the longest $T_{2}$ corresponds to PEG, which is the most mobile part of the particles. 
Shorter $T_{2}$ signals correspond to protons more to the interior of the particles. A signal from sucrose is also visible, as well as two signals that most probably correspond to slow-diffusing particles, because their $T_{2}$ values correspond to those of fast-diffusing particles. Because this intensity appears at the very edge of the correlation map, it is difficult to quantify this fraction in terms of a diffusion coefficient. The slow-diffusing nanoparticle component that was observed by ${ }^{19} \mathrm{~F}$ DOSY only for G5 particles, is visible in DRCOSY maps also of G5 particles only. Mean diffusion coefficients for the fast-diffusing particle fraction, derived from high and low field DRCOSY experiments are presented in Figure 3.3, lower panel, where they are compared with the diffusion coefficients derived from ${ }^{19} \mathrm{~F}$ and ${ }^{1} \mathrm{H}$ DOSY.

\subsection{Discussion}

In this work, we assessed two routes to overcome selectivity issues in NMR nanoparticle diffusometry. The first route relied on ${ }^{19} \mathrm{~F}$ labeling of nanoparticles, leading to background-free diffusion measurements, but lower sensitivity than for ${ }^{1} \mathrm{H}$. The second route deployed ${ }^{1} \mathrm{H}$ DRCOSY, instead of DOSY, to achieve a stronger separation between probe and background signals.

While sensitivity of ${ }^{19} \mathrm{~F}$ DOSY is lower than ${ }^{1} \mathrm{H}$ DOSY due to the limited number of ${ }^{19} \mathrm{~F}$ labels per particle, it is clear from Figure 3.3, upper panel, that error intervals for the fast-diffusing particle fractions are comparable. With the available sensitivity and PFG power, it is not possible to separate the ${ }^{1} \mathrm{H}$ signal from slow-diffusing particles from the ${ }^{1} \mathrm{H}$ signal from the immobile $\kappa$ carrageenan matrix. Although sensitivity of ${ }^{19} \mathrm{~F}$ DOSY is lower due to the limited number of ${ }^{19} \mathrm{~F}$ labels per particle, background-free observation of the particles demonstrates the existence of a slow-diffusing particle fraction for G5 particles only (Huo et al., 2003). In terms of matrix properties, this indicates that the lower limit of the matrix mesh size in the order of 5-7 nm, between the diameters of G3 and G5 particles.

In Figure 3.3, lower panel, it can be seen that the experimental error of DRCOSY for the fastdiffusing particle fraction is higher than the error in a three-component fit of ${ }^{1} \mathrm{H}$ or onecomponent fit of ${ }^{19} \mathrm{~F}$ DOSY. However, like ${ }^{19} \mathrm{~F}$ DOSY, DRCOSY will offer a better separation of probe and background signals in more complex matrices, because signal separation on the basis of $T_{2}$ gives a less complex pattern than on the basis of chemical shift. Although this intensity appears at the edge of the correlation map and therefore is difficult to quantify, it does not appear in $D-T_{2}$ maps of G1 and G3 particles. This indicates that DRCOSY is able to establish the existence of a slow-diffusing particle fraction where ${ }^{1} \mathrm{H}$ DOSY is not. $T_{2}$ offers a much broader separation window than the $\sim 15 \mathrm{ppm}$ of chemical shift commonly observed in ${ }^{1} \mathrm{H}$ NMR. In fact, in the current setup, the NMR spectrum does not offer any significant potential for separation of signals, but rather helps to identify the chemical nature of a component. The advantage of ${ }^{1} \mathrm{H}$ DOSY over DRCOSY in terms of sensitivity thus only exists by the virtue of reliable spectral decomposition, which can only be achieved when fitting a small number of discrete components. 
${ }^{19} \mathrm{~F}$ DOSY and ${ }^{1} \mathrm{H}$ DRCOSY offer clear advantages over ${ }^{1} \mathrm{H}$ DOSY in terms of sensitivity and resolution. These advantages will become more pronounced in compositionally more complex matrices. At the same time, ${ }^{1} \mathrm{H}$ DOSY is complementary to DRCOSY in that it can give information about the chemical nature of the different components, whereas ${ }^{1} \mathrm{H}$ DRCOSY offers a stronger separation power.

Sensitivity is the only limiting factor for nanoparticle diffusometry at low field strengths. With the current ${ }^{19} \mathrm{~F}$ payload, sensitivity at low field strength $(28 \mathrm{MHz})$ is too low at a dosage level of 0.1 wt $\%$. For ${ }^{1} \mathrm{H}$, it can be seen in Figure 3.5, lower panel that the error estimates for high and low field DRCOSY are comparable. Although magnetic field strength differed with a factor of 10 (S/N 30 times lower), the comparable sensitivity can be accounted for by 16 times more signal averaging, the use of a more sensitive RF coil (solenoid instead of birdcage), and by a longer $T_{2}$ for PEG at low field (SI, Figure S3.1d). We have shown the feasibility of DRCOSY nanoparticle diffusometry on (benchtop) low field spectrometers.

\subsection{Conclusions}

${ }^{19} \mathrm{~F}$-labeled dendrimer constructs allow for background-free nanoparticle diffusometry studies. However, the ${ }^{19} \mathrm{~F}$ payload of the current construct ( $\left.7 \mathrm{wt} \%\right)$ requires a high-field NMR instrument to be used for adequate sensitivity. The employed nanoparticle design does allow for higher ${ }^{19} \mathrm{~F}$ payloads without compromising solubility. ${ }^{1} \mathrm{H}$ NMR diffusometry based on observation of the PEG signal offers higher overall sensitivity, but probe and background signals cannot always be adequately separated. In spite of lower overall sensitivity, ${ }^{19} \mathrm{~F}$ DOSY offers superior selectivity and sensitivity for bimodal particle diffusion. As another alternative to ${ }^{1} \mathrm{H}$ DOSY, we demonstrated that ${ }^{1} \mathrm{H}$ DRCOSY has a larger separation power than ${ }^{1} \mathrm{H}$ DOSY at equal overall sensitivity, and that bootstrapping is an effective method to stabilize resulting diffusion- $T_{2}$ maps towards the effects of noise and artifacts. Bimodal nanoparticle diffusion could be established from both ${ }^{19} \mathrm{~F}$ DOSY and ${ }^{1} \mathrm{H}$ DRCOSY. The latter method can be performed using widely available low-field NMR instrumentation.

\section{Supporting Information}

S3.1. Overview of high and low field DRCOSY maps, raw and bootstrap resampled data fits. S3.2. Comparison of ${ }^{1} \mathrm{H}$ and ${ }^{19} \mathrm{~F}$ DOSY spectral decompositions and their standard deviations. S3.3. NMR spectrum of $\kappa$-carrageenan gel in $\mathrm{D}_{2} \mathrm{O}$; presence of sucrose. Background signal of $\kappa$ carrageenan matrix at high PFG amplitude. This material is available free of charge via the Internet at http://doi.org/10.1021/ac502211q.

\section{Acknowledgments}

We acknowledge Gert-Jan Goudappel, Ewoud van Velzen (Unilever R\&D Vlaardingen, NL), and Frank Vergeldt and Alena Průšová (Wageningen University, NL) for their contributions. We 
thank Yi-Qiao Song (Schlumberger-Doll Research, MA, USA) for providing the FLI algorithm. This research received funding from the Netherlands Organization for Scientific Research (NWO) in the framework of the Technology Area COAST. 


\section{References}

Alper, J. S., \& Gelb, R. I. (1990). Standard errors and confidence intervals in nonlinear regression: comparison of Monte Carlo and parametric statistics. Journal of Physical Chemistry, 94(11), 47474751. http://doi.org/10.1021/j100374a068

Axelrod, D., Koppel, D. E., Schlessinger, J., Elson, E., \& Webb, W. W. (1976). Mobility Measurement by Analysis of Fluorescence Photobleaching Recovery Kinetics. Biophysical Journal, 16(9), 1055-1069. http://doi.org/10.1016/S0006-3495(76)85755-4

Berman, P., Levi, O., Parmet, Y., Saunders, M., \& Wiesman, Z. (2013). Laplace inversion of low-resolution NMR relaxometry data using sparse representation methods. Concepts in Magnetic Resonance Part A, 42(3), 72-88. http://doi.org/10.1002/cmr.a.21263

Bernin, D., Goudappel, G.-J., van Ruijven, M., Altskär, A., Ström, A., Rudemo, M., et al. (2011). Microstructure of polymer hydrogels studied by pulsed field gradient NMR diffusion and TEM methods. Soft Matter, 7(12), 5711-5716. http://doi.org/10.1039/c1sm05070b

Braeckmans, K., Peeters, L., Sanders, N. N., De Smedt, S. C., \& DeMeester, J. (2003). Three-Dimensional Fluorescence Recovery after Photobleaching with the Confocal Scanning Laser Microscope. Biophysical Journal, 85(4), 2240-2252. http://doi.org/10.1016/S0006-3495(03)74649-9

Cai, L.-H., Panyukov, S., \& Rubinstein, M. (2011). Mobility of Nonsticky Nanoparticles in Polymer Liquids. Macromolecules, 44(19), 7853-7863. http://doi.org/10.1021/ma201583q

Cheng, Y., Prud'homme, R. K., \& Thomas, J. L. (2002). Diffusion of Mesoscopic Probes in Aqueous Polymer Solutions Measured by Fluorescence Recovery after Photobleaching. Macromolecules, 35(21), 81118121. http://doi.org/10.1021/ma0107758

Colsenet, R., Söderman, O., \& Mariette, F. (2006). Pulsed Field Gradient NMR Study of Poly(ethylene glycol) Diffusion in Whey Protein Solutions and Gels. Macromolecules, 39(3), 1053-1059. http://doi.org/10.1021/ma0519922

Davison, A. C., \& Hinkley, D. V. (1997). Bootstrap Methods and Their Application. Cambridge University Press.

Efron, B. (1979). Bootstrap Methods: Another Look at the Jackknife. The Annals of Statistics, 7(1), 1-26. http://doi.org/10.1214/aos/1176344552

Hagman, J., Lorén, N., \& Hermansson, A.-M. (2010). Effect of Gelatin Gelation Kinetics on Probe Diffusion Determined by FRAP and Rheology. Biomacromolecules, 11(12), 3359-3366. http://doi.org/10.1021/bm1008487

Huo, R., Wehrens, R., van Duynhoven, J., \& Buydens, L. M. C. (2003). Assessment of techniques for DOSY NMR data processing. Analytica Chimica Acta, 490(1-2), 231-251. http://doi.org/10.1016/S00032670(03)00752-9

Liu, M., Nicholson, J. K., \& Lindon, J. C. (1996). High-Resolution Diffusion and Relaxation Edited One- and Two-Dimensional 1H NMR Spectroscopy of Biological Fluids. Analytical Chemistry, 68(19), 33703376. http://doi.org/10.1021/ac960426p

Lorén, N., Shtykova, L., Kidman, S., Jarvoll, P., Nydén, M., \& Hermansson, A.-M. (2009). Dendrimer diffusion in kappa-carrageenan gel structures. Biomacromolecules, 10(2), 275-284. http://doi.org/10.1021/bm801013x

Masaro, L., \& Zhu, X. (1999). Physical models of diffusion for polymer solutions, gels and solids. Progress in Polymer Science, 24(5), 731-775. http://doi.org/10.1016/S0079-6700(99)00016-7

Nilsson, M., \& Morris, G. A. (2007). Improved DECRA processing of DOSY data: correcting for nonuniform field gradients. Magnetic Resonance in Chemistry, 45(8), 656-660. http://doi.org/10.1002/mrc.2023

Provencher, S. W. (1982). CONTIN: A general purpose constrained regularization program for inverting noisy linear algebraic and integral equations. Computer Physics Communications, 27(3), 229-242. http://doi.org/10.1016/0010-4655(82)90174-6 
Rathgeber, S., Beauvisage, H.-J., Chevreau, H., Willenbacher, N., \& Oelschlaeger, C. (2009). Microrheology with Fluorescence Correlation Spectroscopy. Langmuir, 25(11), 6368-6376. http://doi.org/10.1021/la804170k

Salami, S., Rondeau-Mouro, C., van Duynhoven, J., \& Mariette, F. (2013a). PFG-NMR self-diffusion in casein dispersions: Effects of probe size and protein aggregate size. Food Hydrocolloids, 31(2), 248-255. http://doi.org/10.1016/j.foodhyd.2012.10.020

Salami, S., Rondeau-Mouro, C., van Duynhoven, J., \& Mariette, F. (2013b). Probe Mobility in Native Phosphocaseinate Suspensions and in a Concentrated Rennet Gel: Effects of Probe Flexibility and Size. Journal of Agricultural and Food Chemistry, 61(24), 5870-5879. http://doi.org/10.1021/jf304949c

Song, Y. Q., Venkataramanan, L., Hürlimann, M. D., Flaum, M., Frulla, P., \& Straley, C. (2002). T1-T2 Correlation Spectra Obtained Using a Fast Two-Dimensional Laplace Inversion. Journal of Magnetic Resonance, 154(2), 261-268. http://doi.org/10.1006/jmre.2001.2474

Stejskal, E. O., \& Tanner, J. E. (1965). Spin diffusion measurements: Spin echoes in the presence of a timedependent field gradient. The Journal of Chemical Physics, 42, 288-292. http://doi.org/10.1063/1.1695690

Stylianopoulos, T., Diop-Frimpong, B., Munn, L. L., \& Jain, R. K. (2010). Diffusion Anisotropy in Collagen Gels and Tumors: The Effect of Fiber Network Orientation. Biophysical Journal, 99(10), 3119-3128. http://doi.org/10.1016/j.bpj.2010.08.065

van Resandt, R. W. W., Vogel, R. H., \& Provencher, S. W. (1982). Double beam fluorescence lifetime spectrometer with subnanosecond resolution: Application to aqueous tryptophan. Review of Scientific Instruments, 53(9), 1392. http://doi.org/10.1063/1.1137173

Vogel, R. H. (1988). SplMod Users Manual, Version 3. Heidelberg. 



\title{
Complex coacervate core micelles with spectroscopic labels for diffusometric probing of biopolymer networks
}

\begin{abstract}
We present the design, preparation and characterization of two types of complex coacervate core micelles (C3Ms) with cross-linked cores and spectroscopic labels, and demonstrate their use as diffusional probes to investigate the microstructure of percolating biopolymer networks. The first type consists of poly(allylamine hydrochloride) (PAH) and poly(ethylene oxide)poly(methacrylic acid) (PEO-b-PMAA), labeled with ATTO 488 fluorescent dyes. We show that the size of these probes can be tuned by choosing the length of the PEO-PMAA chains. ATTO 488-labeled $\mathrm{PEO}_{113}-\mathrm{PMAA}_{15}$ micelles are very bright with 18 dye molecules incorporated into their cores. The second type is a ${ }^{19} \mathrm{~F}$-labeled micelle, for which we used PAH and a ${ }^{19} \mathrm{~F}$-labeled diblock copolymer tailor-made from poly (ethylene oxide) poly (acrylic acid) $\left(\mathrm{mPEO}_{79}-b-\mathrm{PAA}_{14}\right)$. These micelles contain approximately $4 \mathrm{wt} \%$ of ${ }^{19} \mathrm{~F}$ and can be detected by ${ }^{19} \mathrm{~F}$ NMR. The ${ }^{19} \mathrm{~F}$ labels are placed at the end of a small spacer to allow for the necessary rotational mobility. We used these ATTO- and ${ }^{19} \mathrm{~F}$-labeled micelles to probe the microstructures of a transient gel (xanthan gum) and a cross-linked, heterogeneous gel ( $\kappa$-carrageenan). For the transient gel, sensitive optical diffusometry methods, including fluorescence correlation spectroscopy, fluorescence recovery after photobleaching and super-resolution single nanoparticle tracking allowed us to measure the diffusion coefficient in networks with increasing density. From these measurements, we determined the diameters of the constituent xanthan fibers. In the heterogeneous $\kappa$ carrageenan gels, bimodal nanoparticle diffusion was observed, which is a signpost of microstructural heterogeneity of the network.
\end{abstract}

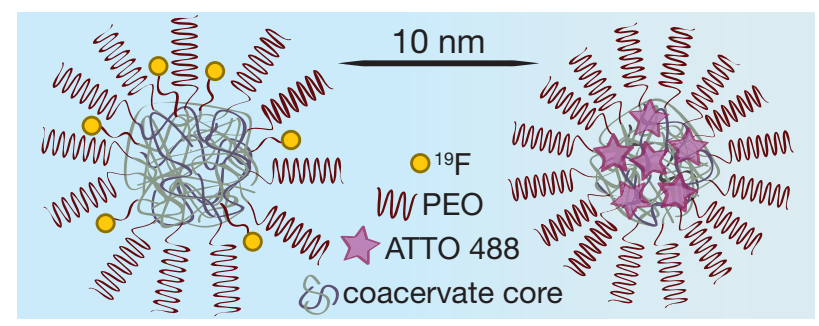

Nadia Bourouina*, Daan W. de Kort*, Freek J.M. Hoeben, Henk M. Janssen, Henk Van As, Johannes Hohlbein, John P.M. van Duynhoven, and J. Mieke Kleijn, Langmuir 2015, 31 (46), pp 12635-12643. DOI: 10.1021/acs.langmuir.5b03496 (*equal contributions) 


\subsection{Introduction}

Biopolymer hydrogels are applied widely, e.g. in cosmetics and food products as texture modifiers (Scholten, Moschakis, \& Biliaderis, 2014). Different microstructures can be obtained depending, amongst others, on the physicochemical properties of the polymers and the kinetics of the gelation process(Stokes, 2012). Measurements of self-diffusion of nanoparticles in biopolymer gels can be used to extract information on the physical properties of the constituent polymer network. Where the mobility of larger colloidal particles reflects the elastic properties of the gel (microrheology) (Mason \& Weitz, 1995), diffusion of smaller particles gives information about the details of gel sub-micron structure (nanoparticle diffusometry) (de Kort, van Duynhoven, Van As, \& Mariette, 2015c; Einhorn-Stoll \& Drusch, 2015; Lorén, Nydén, \& Hermansson, 2009a). Nanoparticle diffusometry has been used in both polysaccharide (Bernin et al., 2011; de Kort, van Duynhoven, Hoeben, Janssen, \& Van As, 2014; Lopez-Sanchez, Schuster, Wang, Gidley, \& Strom, 2015; Lorén, Shtykova, Kidman, Jarvoll, et al., 2009b; Schuster et al., 2014a) and protein (Hagman, Lorén, \& Hermansson, 2010; Salami, Rondeau-Mouro, Barhoum, van Duynhoven, \& Mariette, 2014; Salami, Rondeau-Mouro, van Duynhoven, \& Mariette, 2013; Schuster, Hermansson, Öhgren, Rudemo, \& Lorén, 2014b) gels, or phase-separated mixtures thereof (Wassén et al., 2014).

Both spherical particles and linear polymers such as dextran are sensitive to microstructural features in the gel. Reptation of linear polymeric probes (de Kort, van Duynhoven, Van As, \& Mariette, 2015c; Seiffert \& Oppermann, 2008) prohibits unambiguous translation of their diffusion behavior into structural descriptors of the biopolymer network. In order to utilize spherical particles to that end, they need to be nonsticky with respect to the biopolymer network and have well defined sizes. Only a limited number of categories of spherical nanoparticles can be considered as nonsticky and monodisperse in the 1-50 nm range (de Kort, van Duynhoven, Van As, \& Mariette, 2015c). Well known examples include gold colloidal particles and PEGylated quantum dots. Their intrinsic fluorescence allows for fluorescence correlation spectroscopy (FCS) and single particle tracking (SPT) experiments, but not for fluorescence recovery after photobleaching (FRAP) as they cannot be photobleached. Neither do these nanoparticles contain NMR-active nuclei with sufficient mobility for detection with diffusometric pulsed field gradient (PFG) NMR techniques.

In addition to these types of nanoparticles, we previously presented spherical complex coacervate core micelles (C3Ms) as diffusional nanoparticles (Bourouina, Cohen Stuart, \& Kleijn, 2014). We prepared such $\mathrm{C} 3 \mathrm{Ms}$ by mixing water-soluble neutral-anionic diblock copolymers and cationic polyelectrolytes at the preferred micellar composition (PMC), i.e., near the stoichiometric ratio of the charged groups. In that way self-assembled nanoparticles formed, consisting of a complex coacervate core and a hydrophilic, uncharged corona. The core of the micelles was chemically cross-linked to increase their stability with respect to $\mathrm{pH}$ changes, high salt concentrations and elevated temperatures. In addition, crosslinking prevents dissociation of the C3Ms as potentially 
triggered by interaction with the polymer networks to be studied. The micelles were formed using a commercially available cationic polyelectrolyte already labeled with fluorescein isothiocyanate (FITC). However, FITC showed low brightness and low photo stability. Furthermore its fluorescence quantum yield was strongly affected by the environment of the complex coacervate core, resulting in limited sensitivity (Bourouina et al., 2014; Mahmoudi et al., 2011).

Here we present two novel C3M designs, the first featuring superior fluorescent labels (ATTO 488) for observation by fluorescence microscopy techniques while saving several preparation steps compared to the FITC-C3Ms, the second constituting ${ }^{19} \mathrm{~F}$ moieties for background free diffusometry by ${ }^{19} \mathrm{~F}$ PFG NMR.

We have used our novel nanoparticles for a microstructural study on two polysaccharide hydrogels, xanthan gum and $\kappa$-carrageenan, which we consider model systems. Both are anionic and form highly viscous solutions and gels already at low polymer concentrations. Xanthan gum in aqueous solution is thought to form an ordered network of double helices (Fitzpatrick, Meadows, Ratcliffe, \& Williams, 2013; Ikeda, Gohtani, Nishinari, \& Zhong, 2012; Sato, Kojima, Norisuye, \& Fujita, 1984a; Sato, Norisuye, \& Fujita, 1984b). This structure is promoted through hydrogen bonds and entanglements leading to the formation of a transient network. $\kappa$-Carrageenan forms gels through side-by-side aggregation of helices (Ikeda, Morris, \& Nishinari, 2001), the microstructure of which depends strongly on ionic conditions, particularly the presence of sodium and potassium ions (Hermansson, Eriksson, \& Jordansson, 1991). Of these two systems, $\kappa-$ carrageenan gels have been studied by nanoparticle diffusometry before (de Kort et al., 2014; Hagman, Lorén, \& Hermansson, 2012; Lorén, Shtykova, Kidman, Jarvoll, et al., 2009b), and highly interesting non-Gaussian diffusion of nanoparticles was observed. Given the limited insight into this phenomenon, it is interesting to exploit the larger size regime of the C3Ms presented in this study in the same model gel. The larger size regime should provide greater sensitivity to the network heterogeneity, i.e., a larger fraction of nanoparticles is trapped in regions with a higher network density.

We begin with a detailed description of the design of the ATTO 488 and ${ }^{19} \mathrm{~F}$ labeled micelles. We proceed by using these micelles as diffusional nanoparticles to characterize network density and meso-scale heterogeneity in percolating networks of xanthan gum and $\kappa$-carrageenan. For this purpose we compared the sensitivity of a range of advanced spectroscopic and imaging methods FCS (Haustein \& Schwille, 2007; Koynov \& Butt, 2012; Nolles et al., 2015), total internal reflection single particle tracking (TIRF SPT) (Hohlbein, Gryte, Heilemann, \& Kapanidis, 2010), FRAP (Braeckmans, Peeters, Sanders, De Smedt, \& DeMeester, 2003; Jonasson et al., 2010; Jonasson, Lorén, Olofsson, Nydén, \& Rudemo, 2008) and PFG NMR (Callaghan, 2011)towards observation of the self-diffusion behavior of these particles. 
Design of labeled complex coacervate core micelles. To avoid that the C3Ms aggregate and engage in specific attractive or repulsive interactions with (bio)polymer matrices, we abide by two design principles: firstly, the spectroscopic labels should be completely embedded into the interior of the particles, and, secondly, the outside of the particles should consist of a brush-like poly (ethylene oxide) (PEO) corona. The designs of the ATTO 488 and ${ }^{19} \mathrm{~F}$ labeled micelles are schematically depicted in Figure 4.1.

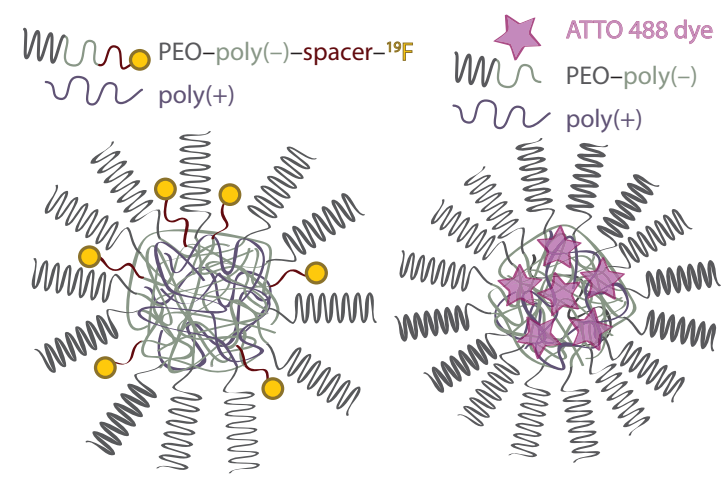

Figure 4.1: Schematic representation of ${ }^{19} \mathrm{~F}$-labeled (left) and ATTO-labeled (right) complex coacervate core micelles. Poly $(-)$ and poly $(+)$ denote negatively and positively charged polyelectrolyte chains, respectively.

We aimed to incorporate the ATTO labels directly into the coacervate cores of the micelles. This was not an option for the ${ }^{19} \mathrm{~F}$ labels, which need rotational freedom to ensure that their NMR relaxation times are on the order of the time scale of the PFG NMR experiment. Therefore the design of the ${ }^{19} \mathrm{~F}$ particles includes a short spacer that allows the labels to move more freely.

For the fluorescent micelles, we started from non-labeled, chemically cross-linked micelles consisting of poly (allylamine hydrochloride) (PAH) and poly (ethylene oxide) poly (methacrylic acid) (PEO-b-PMAA) and covalently incorporated fluorescent labels into the core. Compared to our previous design (Bourouina et al., 2014), we saved several preparation steps which allowed us to label the micelles with a dye of our choice. We used ATTO 488 as a fluorescent label, which is suitable for FCS, TIRF SPT and FRAP as the dye exhibits strong absorption, high fluorescence quantum yield and good photo stability. The resulting fluorescently labeled and cross-linked micelles have a core composed of PMAA blocks, PAH and ATTO 488 dyes, surrounded by a brush-like PEO corona, which ensures solubility in water.

For the ${ }^{19} \mathrm{~F}$-functionalized micelles, we introduced a tailor-made, ${ }^{19} \mathrm{~F}$-labeled mPEO- $b$-PAA diblock copolymer of which the design is shown in Figure 4.2. 


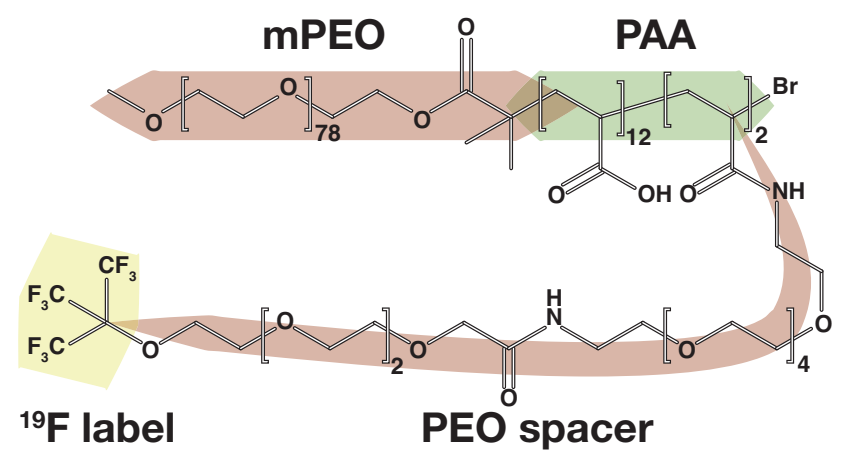

Figure 4.2: Molecular design of the ${ }^{19} \mathrm{~F}$-labeled $\mathrm{mPEO}_{79}-b-\mathrm{PAA}_{14}$ polyelectrolyte. It features two flexible spacers with $-\mathrm{C}\left(\mathrm{C}^{19} \mathbf{F}_{3}\right)_{3}$ end groups. The molecular weight is $6.0 \mathrm{kDa}$. The ${ }^{19} \mathrm{~F}$ labels were tethered to the charged block of the copolymer with short spacers, so that they can protrude from the cores but are still buried in the PEO shell.

\subsection{Materials and Methods}

Preparation of labeled C3Ms. An overview of all chemicals and materials used in our experiments is included in the Supporting Information, section S4.1.

Unlabeled C3Ms. Stable, unlabeled micelles were prepared as described previously.(Bourouina et al., 2014) In short, by mixing PEO-PMAA and PAH aqueous solutions of $\mathrm{pH} 6.5$ at the preferred micellar composition (PMC), micelles form spontaneously through electrostatic interactions. The PMC is at $F^{-}=0.69$, with $F^{-}=\left[n^{-}\right] /\left(\left[n^{-}\right]+\left[n^{+}\right]\right)$, where $\left[n^{-}\right]$and $\left[n^{+}\right]$are the concentrations of carboxylic and amine groups on the polyelectrolyte blocks PMAA and PAH, respectively.

The cores of the micelles are then chemically cross-linked by forming peptide bonds between the carboxylic groups of the PMAA blocks and the amine groups of the PAH by adding a coupling agent, EDAC, and sulfo-NHS. A ratio of 10:1 between the coupling agent and the carboxylic groups was used to crosslink approximately $25 \%$ of the groups. After two hours of incubation, the micellar solution was dialyzed against a $10 \mathrm{mM} \mathrm{NaNO}_{3}$ solution to remove the urea formed during the crosslink reaction.

ATTO 488-labeled C3Ms. ATTO 488 NHS-ester was freshly dissolved in dimethylformamide (DMF) and added to the micellar solution. The final concentration of ATTO 488 dyes in the solution was $0.42 \mathrm{~g} / \mathrm{L}$, corresponding to an excess of ATTO 488 compared to the amine groups ( 20:1). The volume of DMF did not exceed $20 \%$ of the total volume of the sample. The ATTO 488 NHS-ester reacts with the still available amino groups of PAH in the micellar core. The solution was incubated for 48 hours at $\mathrm{pH} 8.3$, which corresponds to the optimum $\mathrm{pH}$ for NHSester coupling. This optimum stems from the fact that with decreasing $\mathrm{pH}$ the degree of 
protonation of the amino groups increases, inhibiting the reaction, while the hydrolysis of the NHS ester increases with increasing $\mathrm{OH}^{-}$concentration. Finally, the micellar solution was dialyzed against $10 \mathrm{mM} \mathrm{NaNO}_{3}$ to remove free ATTO 488 molecules. The fraction of nonincorporated dye and the average fluorescence intensity were monitored by FCS (Supporting Information, section S4.2).

${ }^{19}$ F-labeled C3Ms. First, ${ }^{19} \mathrm{~F}$ groups were tethered to an $\mathrm{mPEO}_{79}-b$ - $\mathrm{PAA}_{14}$ diblock copolymer $\left(M_{n} \approx 6.0 \mathrm{kDa}\right)$, as described in the Supporting Information, section S4.3. The ${ }^{19} \mathrm{~F}$ micelles were prepared in the same way as the unlabeled micelles described above; the PMC for this case was found to be $F^{-}=0.39$.

Preparation of polysaccharide gels. Xanthan gels were prepared by dissolving xanthan gum in a solution of cross-linked ATTO-C3Ms and $10 \mathrm{mM} \mathrm{NaNO}_{3}$ in water. $\kappa$-Carrageenan gels were prepared by dissolving $\kappa$-carrageenan powder, $200 \mathrm{mM} \mathrm{NaCl}, 20 \mathrm{mM} \mathrm{KCl}$ and nanoparticles in water and heating to $80^{\circ} \mathrm{C}$ for 15 minutes.

Characterization of labeled micelles. Particle size of ATTO-labeled micelles was determined by dynamic light scattering (DLS). Their shape was visualized by cryo-TEM. Fluorescence lifetimes of the incorporated ATTO-dyes were measured with time-correlated single photon counting (TCSPC). The NMR relaxation times of the ${ }^{19} \mathrm{~F}$-labeled micelles were determined by NMR relaxometry at $B_{0}=7.0 \mathrm{~T}$. The experimental details of these four methods are included in the Supporting Information, section S4.4.

Nanoparticle diffusometry. We use four different diffusometric methods to track the mobility of our C3Ms in polysaccharide gels. FCS, FRAP and total internal reflection fluorescence (TIRF) superresolution single particle tracking (SPT) are used to measure self-diffusion coefficients of the ATTO-labeled particles. ${ }^{1} \mathrm{H}$ and ${ }^{19} \mathrm{~F}$ PFG NMR is used to measure self-diffusion of the ${ }^{19} \mathrm{~F}$ labeled particles. The concentration of labeled micelle dissolved in the hydrogels used was different for each method: $0.1 \mathrm{wt} \%$ for FRAP, $0.5 \cdot 10^{-3} \mathrm{wt} \%$ for FCS, $0.2 \cdot 10^{-3} \mathrm{wt} \%$ for SPT and $0.1 \mathrm{wt} \%$ for PFG NMR. Further experimental details are included in the Supporting Information, section S4.5.

\subsection{Results and Discussion}

\subsubsection{Characterization of the labeled micelles}

Micellar size and shape. Particle size is a critical parameter in obstruction-effect and hydrodynamic models of diffusion used to extract network parameters(Hadjiev \& Amsden, 2015; Masaro \& Zhu, $1999)$ as the relative size of particles and network meshes determine the level of restriction of 
particle diffusion. Varying particle size therefore provides important information about network density and heterogeneity.

The size of non-cross-linked micelles can be tuned by varying the size of the negatively charged polyelectrolyte chains, as shown in Figure 4.3 for PEO-PMAA of different lengths (the size of the homopolymer PAH was kept constant).

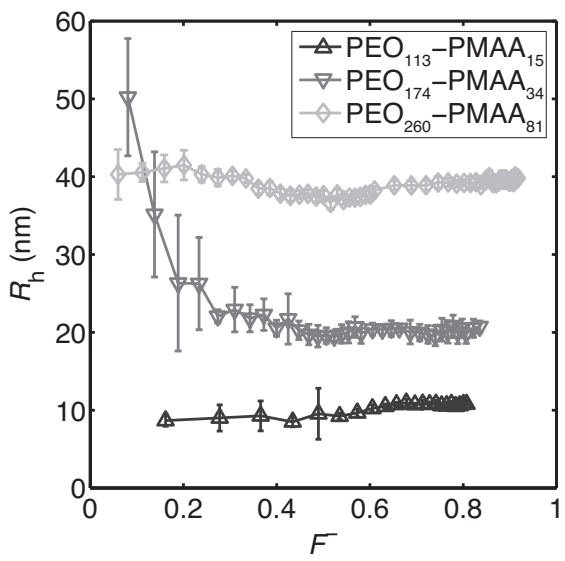

Figure 4.3 Hydrodynamic radius $R_{\mathrm{h}}$ of non-cross-linked micelles as a function of composition $F^{-}$for different sizes of the negatively charged polyelectrolyte PEO-PMAA, determined by DLS. The length of the positively charged polyelectrolyte $\mathrm{PAH}$ was not varied. Background electrolyte concentration was $10 \mathrm{mM} \mathrm{NaCl}$. Error bars indicate the standard deviation in the measurements (generally 10 repetitions).

Cryo-TEM images of the micelles, presented in Figure 4.4a, allow a direct observation of their size and shape.

The micelles appear circular on the 2D image suggesting a spherical shape. The PEO corona of the micelles is not dense enough to be observed with this technique; only the complex coacervate core is visible. This is the reason that the micelles appear relatively small $(R=9 \pm 1 \mathrm{~nm}$ in this particular image). We attached ATTO-488 labels to the crosslinked micelles prepared with $\mathrm{PEO}_{113}-b$ PMAA $_{15}$ at the PMC, i.e., $F^{-}=0.69$ (cf. Figure 4.3). For these crosslinked ATTO-labeled micelles, we found a hydrodynamic radius of $13 \pm 2 \mathrm{~nm}$ from the diffusion coefficient of ATTO-C3Ms in water at room temperature as determined by FCS (using the Stokes-Einstein relation) The FCS autocorrelation curve of the ATTO-C3Ms in water shown in Figure $4.4 \mathrm{~b}$ is described very well with a one-component model, implying that the particle size distribution is narrow. 

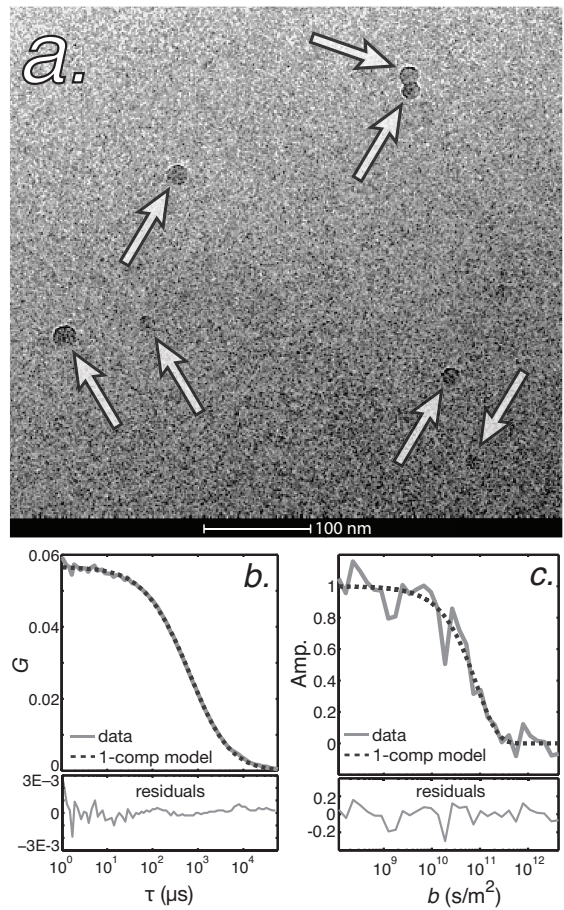

Figure 4.4: ( $\boldsymbol{a}$ ) Cryo-TEM image of ATTO-C3Ms (scale bar $100 \mathrm{~nm}$ ). (b) FCS autocorrelation curve $\left(10^{0}-10^{5} \mu \mathrm{s}\right)$ of ATTO-C3Ms in water fitted to a single-component diffusion model (cf. SI section S4.5 eq. 1) corresponding to a diffusion coefficient of $1.6 \pm 0.2 \cdot 10^{-11} \mathrm{~m}^{2} / \mathrm{s}\left(R_{\mathrm{h}} 13 \pm 2 \mathrm{~nm}\right)$. (c) ${ }^{19} \mathrm{~F}$ PFG NMR signal attenuation curve of ${ }^{19} \mathrm{~F}-\mathrm{C} 3 \mathrm{Ms} ; b$ is proportional to the applied magnetic field gradient amplitude according Stejskal-Tanner equation (Stejskal \& Tanner, 1965) (cf. SI section S4.5 eq. 2). The curve can be described with a single component corresponding to a diffusion coefficient of $1.4 \pm$ $0.1 \cdot 10^{-11} \mathrm{~m}^{2} / \mathrm{s}\left(R_{\mathrm{h}} 15 \pm 1 \mathrm{~nm}\right)$.

For the ${ }^{19} \mathrm{~F}$-labeled micelles, the PMC was found at composition $F^{-}=0.39$ by means of DLS (Supporting Information section S4.6). For this composition we calculated the mass fraction ${ }^{19} \mathrm{~F}$ in the micelles to be $4 \mathrm{wt} \%$. This value is comparable to the ${ }^{19} \mathrm{~F}$ loading of (smaller) ${ }^{19} \mathrm{~F}$-labeled dendrimers that we recently described elsewhere.(de Kort et al., 2014; de Kort, Rombouts, Hoeben, Janssen, Van As, et al., 2015b) A ${ }^{19} \mathrm{~F}$ PFG NMR measurement on crosslinked ${ }^{19} \mathrm{~F}$-C $3 \mathrm{Ms}$ in water is presented in Figure 4.4c. The signal attenuation curve is mono-exponential, indicating that the particles are monodisperse in size. From the diffusion coefficient, the Stokes-Einstein relation gives a hydrodynamic radius of $15 \pm 1 \mathrm{~nm}$ for crosslinked ${ }^{19} \mathrm{~F}-\mathrm{C} 3 \mathrm{Ms}$, similar to the size of the crosslinked ATTO-C3Ms $\left(R_{\mathrm{h}}=13 \pm 2 \mathrm{~nm}\right)$ and non-labeled crosslinked micelles (Bourouina et al., 2014) $\left(R_{\mathrm{h}}=14 \mathrm{~nm}\right)$. 
Stability of the labeled C3Ms. For the ATTO-C3Ms, the fact that the labels are built into the micellar cores might affect the overall stability of the micelles towards changing $\mathrm{pH}$ and ionic strength. We have studied the stability of the C3Ms towards changing $\mathrm{pH}$ and ionic strength by DLS. The ATTO-C3Ms turn out to be stable (i.e., they do not fall apart) over the whole salt $(\mathrm{NaCl})$ concentration and $\mathrm{pH}$ range studied (Figures $4.5 \mathrm{a}$ and $\mathrm{b}$ ), although the micelles do change somewhat in size with changing conditions.

These results show that the presence of the dyes in the micellar cores does not negatively affect their stability. It is noted that in these plots the particle radii are larger than the earlier mentioned value of $R_{\mathrm{h}}(\sim 14 \mathrm{~nm})$, probably due to differences in preparation procedure: for DLS titration the concentration of one polymer was progressively added to the other polymer. The micelles used in the stability measurements were prepared by directly mixing the polymers at a ratio corresponding to the PMC after which the micellar solutions were filtered.

Since for the ${ }^{19} \mathrm{~F}-\mathrm{C} 3 \mathrm{Ms}$ the labels do not compete for binding positions within the core, we were less concerned about compromised stability and verified their stability only with respect to $\mathrm{pH}$ (Supporting Information section S4.7).

We also investigated the effect of temperature on the stability of the ATTO-C3M bonds by FCS. This is of specific importance because diffusional nanoparticles are typically added to a polymeric solution that needs a heating step to induce gelation. In case of the ${ }^{19} \mathrm{~F}-\mathrm{C} 3 \mathrm{Ms}$, the ${ }^{19} \mathrm{~F}$ labels are tethered covalently to the negatively charged polyelectrolyte before the micelles are prepared, which differs from the situation for the ATTO-C3Ms. Therefore, we only checked the temperature stability of the latter. We gradually heated the ATTO-C3M micellar solutions to temperatures up to $90{ }^{\circ} \mathrm{C}$ and allowed the solutions to cool down to room temperature. Subsequently, the fluorescence brightness per micelle and the diffusion coefficient were obtained with FCS. The values were found to be independent of the heating treatment (Figure 4.5c), showing that there is no leakage of dyes and that the micelles as a whole are stable up to at least 90 ${ }^{\circ} \mathrm{C}$. 

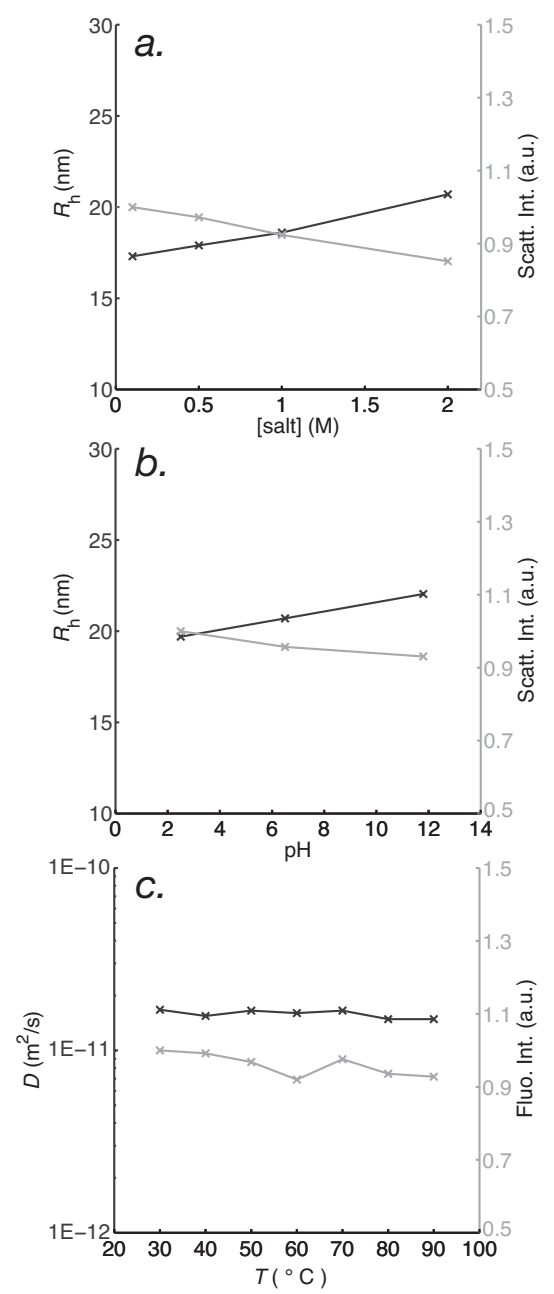

Figure 4.5: Stability of ATTO-C3Ms. Effect of $(\boldsymbol{a})$ the $\mathrm{NaCl}$ concentration (at $\mathrm{pH} 6.5$ ) and (b) the $\mathrm{pH}$ (at $10 \mathrm{mM} \mathrm{NaCl}$ ) on the scattered intensity and hydrodynamic radius from DLS (data points are the average of 10 repeated measurements; standard deviation $<1 \mathrm{~nm}$ ). (c) Effect of the temperature of a heating treatment on the diffusion coefficient and fluorescence brightness per micelle measured by FCS (data collected at room temperature). Black symbols and lines refer to the left axis, grey symbols and lines to the right.

Fluorescence properties of the ATTO-C3Ms. The normalized steady-state absorption spectra of free ATTO 488 and ATTO-C3Ms in water together with their emission spectra (excitation at $480 \mathrm{~nm}$ ) are shown in Figures 4.6a and b, respectively. 

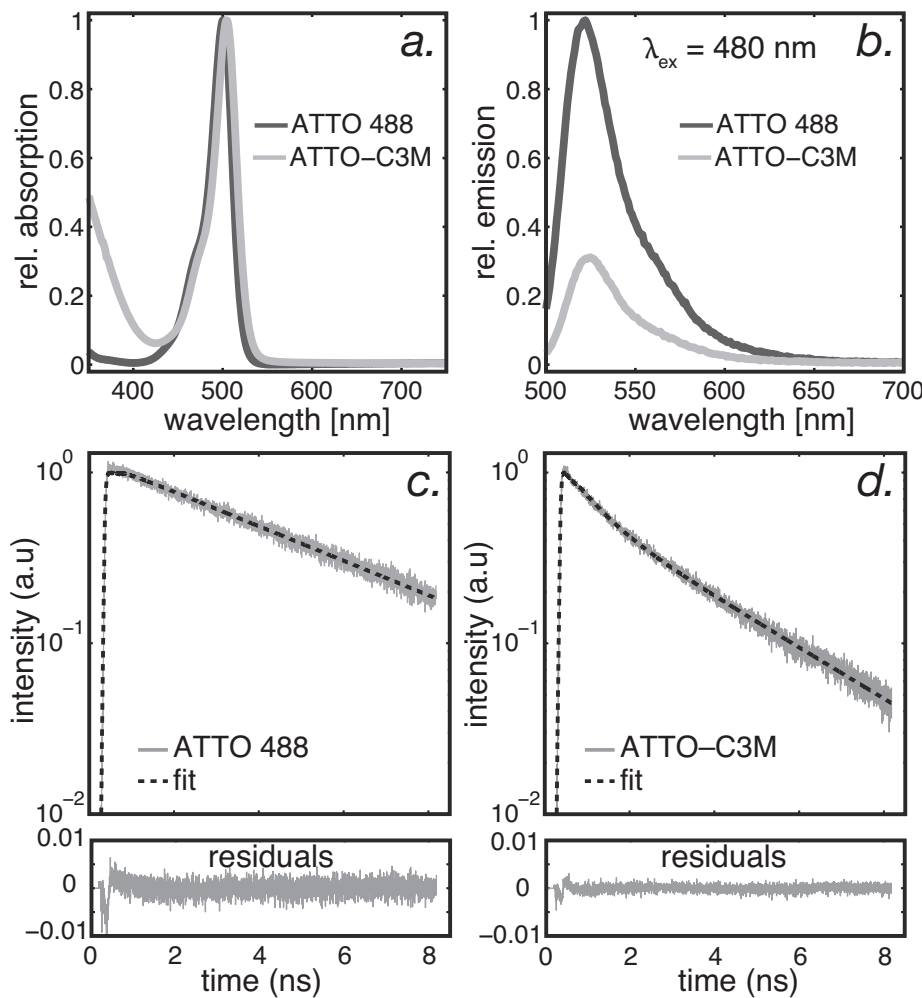

Figure 4.6: $(\boldsymbol{a})$ Normalized absorption spectra of free ATTO 488 molecules and ATTO-C3Ms. (b) Fluorescence emission spectra (excitation at $480 \mathrm{~nm}$ ), normalized to the emission of free ATTO 488 at identical absorption. The reduced emission of the ATTO-C3Ms relative to the free ATTO dye is due to quenching. (c) Fluorescence decay trace of "free" ATTO 488 in water (mono-exponential). (d) Fluorescence decay trace of ATTO-C3Ms in water (bi-exponential).

We observe that there is only a very small shift of $\sim 1 \mathrm{~nm}$ in the absorption maximum due to the incorporation of the dyes into the micelles. The emission spectra were recorded for samples with identical absorption at $480 \mathrm{~nm}$, so that we can infer the relative quantum yield by looking at the relative fluorescence emission intensities. At the emission maximum, the emission of ATTO-C3M relative to free ATTO is $0.31 \pm 0.01$. Apparently, fluorescence of the dye in the core is quenched due to a different environment compared to the bulk solution and/or the proximity of other ATTO labels; hence, we proceed to determine the number of labels incorporated in each micelle.

Number of ATTO labels per micelle. We used FCS to determine the number of dyes incorporated into each micelle. We compared the fluorescence intensity of free ATTO 488 and ATTO-C3Ms at identical laser power and found relative brightness values of $1.0 \pm 0.1$ and $5.7 \pm 0.5$ counts per 
molecule/particle per unit of time, respectively. Correcting for $31 \pm 1 \%$ quantum yield of ATTO 488 in the ATTO-C3Ms relative to free dye (as determined from the steady state emission spectra), this leads to a number of $18 \pm 3$ dye molecules per micelle. Despite of significant quenching of fluorescence in the ATTO-C3Ms, their total brightness is much higher than that of the free dye molecule due to the large number of incorporated labels.

TCSPC. To determine the origin of the fluorescence quenching in the ATTO-C3Ms, we measured fluorescence lifetimes by TCSPC. Decay traces for ATTO 488 in bulk solution and ATTO-C3Ms are presented in Figures 4.6c and d. ATTO 488 fluorescence decays with a single lifetime of $4.30 \pm$ $0.08 \mathrm{~ns}$, whereas ATTO-C3M fluorescence decays with two apparent lifetimes of $2.90 \pm 0.05 \mathrm{~ns}$ (54\%) and $0.65 \pm 0.03 \mathrm{~ns}(46 \%)$. The low relative quantum yield in the ATTO-C3Ms (31\%) as determined from the steady-state emission spectra is reflected in the shorter lifetimes as observed by TCSPC. Possibly, the shortest lifetime in the micelles corresponds to a population of dye molecules in close proximity to each other, leading to concentration quenching.

Longitudinal $\left(T_{1}\right)$ and transverse $\left(T_{2}\right)$ relaxation parameters of ${ }^{19} \mathrm{~F}-\mathrm{C} 3 \mathrm{Ms}$. For PFG NMR, the longitudinal $\left(T_{1}\right)$ and transverse $\left(T_{2}\right)$ NMR relaxation times should be on the relevant time scale for the PFG NMR experiment, which is typically between 10 and $1000 \mathrm{~ms}$; The NMR signal of ${ }^{19} \mathrm{~F}$ labels with relaxation times $<10 \mathrm{~ms}$ would fully relax during the experiment. For the $-\mathrm{C}\left(\mathrm{C}^{19} \mathbf{F}_{3}\right)_{3}$ moieties we measured $T_{1}=620 \pm 20 \mathrm{~ms}$ and $T_{2}=11 \pm 1 \mathrm{~ms}$ (at $282 \mathrm{MHz}$ resonance frequency). In simple aqueous solutions, the ${ }^{19} \mathrm{~F}-\mathrm{C} 3 \mathrm{Ms}$ can also be observed by ${ }^{1} \mathrm{H} \mathrm{NMR}$ using the ${ }^{1} \mathrm{H}$ signal from the PEO corona (3.6 ppm) provided that the signal of water is adequately suppressed, for which we use a pair of gradient pulses. At $300 \mathrm{MHz}, T_{1}(\mathrm{PEO})=507 \pm 25 \mathrm{~ms}$ and $T_{2}(\mathrm{PEO})=343 \pm 34$ ms. This means that the current particle design allows for PFG NMR experiments on the $10-500$ $\mathrm{ms}$ (diffusion) timescale, for both ${ }^{1} \mathrm{H}$ and ${ }^{19} \mathrm{~F}$. The $T_{2}$ relaxation times of the ${ }^{19} \mathrm{~F}$ groups in ${ }^{19} \mathrm{~F}-$ labeled dendrimers (de Kort et al., 2014) are significantly longer ( 10 times) than in the C3Ms, due to the relatively high mobility of the dendritic ${ }^{19} \mathrm{~F}$ groups. This difference most likely relates to the overall particle size, as a larger particle size leads to slower tumbling and therefore a lower molecular mobility.

With our current design of ${ }^{19} \mathrm{~F}-\mathrm{C} 3 \mathrm{Ms}$, nanoparticle diffusometry by ${ }^{1} \mathrm{H}$ PFG NMR gives a higher signal-to-noise ratio than ${ }^{19} \mathrm{~F}$ PFG NMR because of the higher intensity of the ${ }^{1} \mathrm{H}$ signal of PEO relative to the ${ }^{19} \mathrm{~F}$ signal of $-\mathrm{C}\left(\mathrm{CF}_{3}\right)_{3}$. However, ${ }^{1} \mathrm{H}-\mathrm{NMR}$ leads to complications when the polymer network/matrix under study gives interfering or overlapping ${ }^{1} \mathrm{H}$ NMR signals. The ${ }^{1} \mathrm{H}$ experiment can used only in relatively "clean" matrices in which the background signal can be fully suppressed, as is the case in $\kappa$-carrageenan gels as described below. We note that the current ${ }^{19} \mathrm{~F}$ labeled mPEO-b-PAA polyelectrolyte design can be adapted to allow for increased internal 
mobility of the labels (i.e., by increasing the length of the spacers), which would lengthen the ${ }^{19} \mathrm{~F}$ transversal relaxation time.

\subsubsection{Observing biopolymer network density and heterogeneity by nanoparticle diffusometry}

We have used our two new micellar designs for microstructural studies in two polysaccharide gels (xanthan gum and $\kappa$-carrageenan). In these model systems, we expect to observe a single diffusion coefficient in the transient gel (xanthan gum) and non-Gaussian diffusion due to particle restriction in $\kappa$-carrageenan. This choice of model systems allows us to compare results between various diffusometric methods.

Diffusometry in xanthan gum. Xanthan gum is a polysaccharide forming transient gels at low concentrations, which has been reported to form double-helical strands with a thickness of 2.0 $2.5 \mathrm{~nm}$ (Sato, Kojima, Norisuye, \& Fujita, 1984a; Sato, Norisuye, \& Fujita, 1984b). We have measured diffusion coefficients of ATTO-C3Ms in various xanthan gum solutions up to concentrations of $1.0 \mathrm{wt} \%$; in all cases we found a single diffusion coefficient, which decreases with polymer concentration (Figure 4.7).

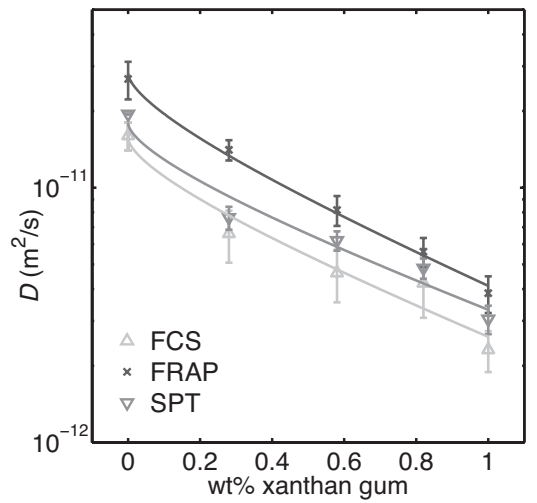

Figure 4.7 Diffusion coefficients of ATTO-C3Ms in xanthan gum. Solid lines represent a fit to the obstruction model of nanoparticle diffusion in polymer gels by Johnson et al. (Johnson, Berk, Jain, \& Deen, 1996)

The diffusion coefficients as determined by the various methods slightly differ but show the same trends as a function of polymer concentration. In case of FRAP it may be that the high number of bleaching steps (>10 steps due to the high brightness of the particles and consequently long bleaching time) needed compromises the determination of the diffusion coefficients because FRAP models assume the bleaching step to be instantaneous. For FCS, on the other hand, the 
high particle brightness is advantageous since it causes strong intensity fluctuations when particles diffuse in and out of the confocal volume. In TIRF SPT, the micelles appear as bright spots in the TIRF microscopy images (Figure 4.8).

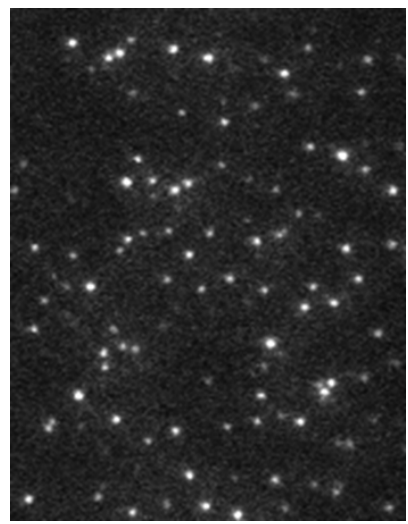

Figure 4.8: TIRF microscopy image of an aqueous solution of ATTO-C3Ms (pixel size $130 \cdot 130 \mathrm{~nm}$ ).

The size of each pixel is $130 \cdot 130 \mathrm{~nm}$ and individual fluorescent spots appear larger than a single pixel due to the diffraction limit, but we can fit a two-dimensional Gaussian intensity profile to the spots in order to localize the particles with sub-pixel accuracy. By tracking the positions of individual micelles frame by frame, we determine diffusion coefficients from a histogram of meansquare displacements. The diffusion coefficient of ATTO-C3Ms in water by SPT $\left(1.9 \pm 0.2 \cdot 10^{-11}\right.$ $\mathrm{m}^{2} / \mathrm{s}$ ) differs not significantly from the value determined by FCS and also the diffusion coefficients as a function of xanthan volume fraction follow the same trend as observed by the other methods. We can use the concentration dependency of the micellar diffusion coefficient to infer an average polymer strand size. Johnson et al. (Johnson et al., 1996) proposed the following model based on obstruction-effect and hydrodynamic arguments:

$D_{\varphi}=D_{0} \exp \left(-0.84 \alpha^{1.09}\right)\left[1+\left(\frac{R_{h}^{2}}{\beta}\right)^{1 / 2}+\frac{1}{3} \frac{R_{h}^{2}}{\beta}\right]^{-1}$,

where $\alpha=\varphi\left(\frac{R_{h}+R_{f}}{R_{f}}\right)$ and $\beta=0.31 R_{f}^{2} \varphi^{-1.17}$.

$D_{0}$ is the particle diffusion coefficient in water, $\varphi$ the polymer volume fraction, $R_{\mathrm{h}}$ the hydrodynamic radius of the probe particle and $R_{f}$ the fiber radius. By describing our data with this model, we find a fiber diameter $\left(2 R_{f}\right)$ of $2.0 \pm 0.4 \mathrm{~nm}$ for all three fluorescence methods used. This value is in agreement with the strand thickness of the xanthan double helix as determined by Sato et al. (Sato, Kojima, Norisuye, \& Fujita, 1984a; Sato, Norisuye, \& Fujita, 1984b) We conclude that 
the fluorescent micelles are ideally suited as fluorescent probes, allowing us to turn to the more complicated case of diffusion in the strongly heterogeneous network of $\kappa$-carrageenan gels.

Diffusion in $\kappa$-carrageenan gels. $\kappa$-Carrageenan forms strongly cross-linked heterogeneous gels under $\mathrm{Na}^{+} / \mathrm{K}^{+}$conditions. In our earlier work (de Kort et al., 2014) and in a study by Lorén et al. (Lorén, Shtykova, Kidman, Jarvoll, et al., 2009b), it was shown that under these conditions, the heterogeneity is reflected in the diffusion behavior of dendritic $\left(R_{\mathrm{h}} \approx 3 \mathrm{~nm}\right)$ nanoparticles, of which the diffusion could no longer be described by a single diffusion coefficient. Two discrete diffusion coefficients were observed from PFG NMR measurements on dendrimers in $\kappa$ carrageenan, the slowest-diffusing component belonging to a fraction of less than a few percent of embedded particles. In case this fraction would represent particles that become entrapped in "cages" formed by the rigid $\kappa$-carrageenan fibers, the slow-diffusing (or rather immobilized) fraction should become more significant for larger particles such as our ATTO- and ${ }^{19} \mathrm{~F}-\mathrm{C} 3 \mathrm{Ms}$. Diffusion coefficients of C3Ms in $\kappa$-carrageenan are presented in Figure 4.9.

As seen in Figure 4.9a, we observe bimodal diffusion (a "fast" and a "slow" fraction) by FRAP and ${ }^{1} \mathrm{H}$ PFG NMR, but only a single fraction with FCS and ${ }^{19} \mathrm{~F}$ PFG NMR. We will now discuss why these differences arise.

While FCS can, in principle, detect bimodal diffusion, both diffusion coefficients should be large enough for the particles to move through the confocal spot before they are bleached. In the case of $\kappa$-carrageenan, part of the particles diffuses very slowly on the experimental time scale so that they are bleached and only a single "fast" fraction is observed.

We also find only a single diffusion coefficient for the ${ }^{19} \mathrm{~F}-\mathrm{C} 3 \mathrm{Ms}$ by ${ }^{19} \mathrm{~F}$ PFG NMR (attenuation curves presented in the Supporting Information, Figure S4.8.1). The fact that ${ }^{19} \mathrm{~F}$ PFG NMR is not able to observe a "slow" fraction is most probably due to the relatively short $T_{2}$ relaxation time $(\sim 10 \mathrm{~ms})$ of the ${ }^{19} \mathrm{~F}$ labels. It is plausible that the slow fraction observed by ${ }^{1} \mathrm{H}$ PFG NMR and FRAP has a shorter $T_{2}$ time than the fast fraction, leading to the effective disappearance of the slow fraction from the ${ }^{19} \mathrm{~F}$ spectrum. From a ${ }^{1} \mathrm{H}$ diffusion- $T_{2}$ correlation (DRCOSY) experiment, we observe that the ${ }^{1} \mathrm{H} \mathrm{T}_{2}$ time of the slow-diffusion fraction is to slightly shorter than that of the fast diffusing fraction ( $c f$. Supporting Information, Figure S4.9.1). For the ${ }^{19} \mathrm{~F}$ labels, whose $T_{2}$ is already short compared to the time scale of the PFG NMR experiment, a similar decrease would lead to effective disappearance of the signal.

Although it should be possible to observe bimodal diffusion by TIRF SPT, we did not succeed to get good results because immobilization of $\mathrm{C} 3 \mathrm{Ms}$ resulted in too high background fluorescence.

With ${ }^{1} \mathrm{H}$ PFG NMR, both fast and slow fractions are observed. At low $\kappa$-carrageenan concentrations, error margins of the diffusion coefficients are large, because the fraction of the slow-diffusing component is very small as can be seen in Figure $4.9 \mathrm{~b}$. The "fast" component overlaps with the ${ }^{19} \mathrm{~F}$ PFG NMR and FCS results presented before. For the fast-diffusing fraction, 
Johnson's diffusion model (red curve in Figure 4.9a) yields a polymer strand thickness of 7.2 \pm 1.0 $\mathrm{nm}$, which is thicker than the $2.0 \pm 0.4$ in xanthan gels, reflecting the side-by-side stacking of the polymer chains in carrageenan gels.

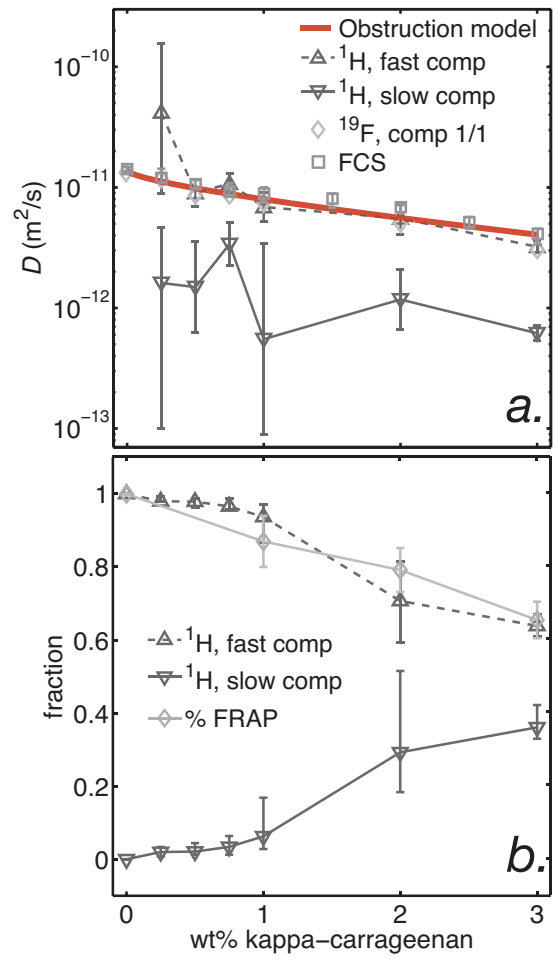

Figure 4.9: (a) Diffusion coefficients of ${ }^{19} \mathrm{~F}-\mathrm{C} 3 \mathrm{Ms}$ measured by FCS, ${ }^{19} \mathrm{~F}$ PFG NMR and ${ }^{1} \mathrm{H}$ PFG NMR in $\kappa$-carrageenan. With FCS and ${ }^{19}$ F PFG NMR we find only a single diffusion coefficient; with ${ }^{1} \mathrm{H}$ PFG NMR an additional slow-diffusing component. (b) Fractions of fast- and slow-diffusing components as measured by ${ }^{1} \mathrm{H}$ PFG NMR compared to immobile fractions as determined from FRAP.

The amplitude of the slow fraction increases significantly with polymer concentration. As can be seen in Figure 4.9b, the mobile fractions obtained by FRAP data closely follow those obtained by ${ }^{1} \mathrm{H}$ PFG NMR. The time scale of the FRAP experiment is $\sim 10^{2}$ times longer than the PFG NMR experiment. This implies that the ${ }^{1} \mathrm{H}$ PFG NMR experiment overestimates the diffusion coefficients of the slow fraction, which might in reality be fully immobilized. This over-estimation is due to the limited magnetic field gradient amplitude of the NMR setup, which sets a lower limit to the diffusion coefficients that can be accurately determined. With FRAP, on the other hand, we were not able to determine diffusion coefficients of the "fast" fraction due to the large offset in the FRAP curves. 
The increase of the amplitude of the slow fraction is much stronger than observed in earlier work (de Kort et al., 2014; Lorén, Shtykova, Kidman, Jarvoll, et al., 2009b). This difference might be due to the four times larger size of the labeled C3Ms as compared to the dendritic particles used in earlier studies. In case of the dendrimers, a slow-diffusing fraction was seen only for $R_{\mathrm{h}}=3.5 \mathrm{~nm}$ particles at much lower intensities, and could not be observed for $R_{\mathrm{h}}=1.4 \mathrm{~nm}$ and $2.3 \mathrm{~nm}$ particles (de Kort et al., 2014). This can be rationalized by considering the biopolymer network as a heterogeneous "sieve", through which the particles have to pass when moving between two points in the system. With increasing polymer concentration, particles will experience stronger sieving (Lorén, Nydén, \& Hermansson, 2009a). Stronger sieving will also occur with increasing particle size. It thus turns out that the larger C3Ms are more sensitive towards these microstructural features in $\kappa$-carrageenan networks. We note that the "sieving" explanation of the bimodal diffusion is still partly conjectural and needs to be reconciled with the lack of apparent diffusionobservation time dependency of the PFG NMR attenuation curve, and lack of apparent diffusive exchange between the two fractions (de Kort, Hoeben, Janssen, Bourouina, Kleijn, et al., 2015a).

\subsection{Conclusions}

We designed and prepared two types of nanoparticles for diffusometric probing of biopolymer networks: bright and stable ATTO 488-labeled fluorescent complex coacervate core micelles, which were found to have about 18 dye molecules incorporated into their cores, and ${ }^{19} \mathrm{~F}$-labeled complex coacervate core micelles for background free ${ }^{19} \mathrm{~F}$ PFG NMR diffusometry, prepared by using a tailor-made ${ }^{19} \mathrm{~F}$-labeled diblock copolymer. The ${ }^{19} \mathrm{~F}$ labels were placed at the end of a small spacer to allow for the necessary rotational mobility; the ${ }^{19} \mathrm{~F}$ content of the micelles amounts to 4 $\mathrm{wt} \%$. We used both types of nanoparticles for probing the microstructures of a transient (xanthan gum) gel and cross-linked, heterogeneous ( $\kappa$-carrageenan) gel, which we consider model systems. For xanthan gum, sensitive fluorescence methods (FCS, FRAP and SPT) allowed us to measure the diffusion coefficient in networks with increasing density, from which the diameter of the constituent xanthan fibers was inferred. For $\kappa$-carrageenan gels, we found bimodal diffusion of nanoparticles. The fraction of "slow" particles increases with polymer concentration and is much more prominent than for smaller dendritic particles used in earlier work (de Kort et al., 2014; Lorén, Shtykova, Kidman, Jarvoll, et al., 2009b). FCS, FRAP, and PFG NMR diffusometric methods could detect the dominant fraction. The slowly diffusing fraction could be detected by FRAP and ${ }^{1} \mathrm{H}$ PFG NMR; ${ }^{19} \mathrm{~F}$ NMR detection turned out to be compromised by the limited rotational mobility of the labels. We expect that this issue can be solved by adapting the design of the nanoparticles, by using a longer spacer in the tailor-made ${ }^{19} \mathrm{~F}$-labeled diblock copolymer.

\section{Supporting Information}

S4.1. Overview of chemicals and materials. S4.2. Purification of the ATTO-C3Ms by dialysis. S4.3. Synthesis of mPEO- $b$-PAA $\left({ }^{19} \mathrm{~F}\right)$. S4.4. Characterization of labeled micelles: details of 
experimental procedures. S4.5. Nanoparticle diffusometry: details of experimental procedures. S4.6. Preferred micelle composition of ${ }^{19} \mathrm{~F}-\mathrm{C} 3 \mathrm{Ms}$. S4.7. Stability of ${ }^{19} \mathrm{~F}-\mathrm{C} 3 \mathrm{Ms}$ towards $\mathrm{pH}$. S4.8. PFG NMR: ${ }^{19} \mathrm{~F}$ and ${ }^{1} \mathrm{H}$ DOSY signal attenuation curves. S4.9. PFG NMR: Diffusion relaxation correlation $\left(\mathrm{D}-\mathrm{T}_{2}\right)$ spectroscopy (DRCOSY). This material is available free of charge via the Internet at http://doi.org/10.1021/acs.langmuir.5b03496.

\section{Acknowledgments}

We acknowledge Ellen Drost, Maria Sovago, Gerard van Dalen (Unilever R\&D, Vlaardingen, NL), Emilie Wientjes, Arjen Bader, Adrie Westphal and Remco Fokkink (Wageningen University, NL) for technical assistance. This research received funding from the Netherlands Organization for Scientific Research (NWO) in the framework of the Technology Area COAST. 


\section{References}

Bernin, D., Goudappel, G.-J., van Ruijven, M., Altskär, A., Ström, A., Rudemo, M., et al. (2011). Microstructure of polymer hydrogels studied by pulsed field gradient NMR diffusion and TEM methods. Soft Matter, 7(12), 5711-5716. http://doi.org/10.1039/c1sm05070b

Bourouina, N., Cohen Stuart, M. A., \& Kleijn, J. M. (2014). Complex coacervate core micelles as diffusional nanoprobes. Soft Matter, 10(2), 320-331. http://doi.org/10.1039/c3sm52245h

Braeckmans, K., Peeters, L., Sanders, N. N., De Smedt, S. C., \& DeMeester, J. (2003). Three-Dimensional Fluorescence Recovery after Photobleaching with the Confocal Scanning Laser Microscope. Biophysical Journal, 85(4), 2240-2252. http://doi.org/10.1016/S0006-3495(03)74649-9

Callaghan, P. T. (2011). Translational Dynamics \& Magnetic Resonance. New York: Oxford University Press.

de Kort, D. W., Hoeben, F. J. M., Janssen, H. M., Bourouina, N., Kleijn, J. M., van Duynhoven, J. P. M., \& Van As, H. (2015a). 19F Labelled Polyion Micelles as Diffusional Nanoprobes. In F. Capozzi, L. Laghi, \& P. S. Belton (Eds.), Magnetic Resonance in Food Science. Cambridge, UK: The Royal Society of Chemistry. http://doi.org/10.1039/9781782622741-00109

de Kort, D. W., Rombouts, W. H., Hoeben, F. J. M., Janssen, H. M., Van As, H., \& van Duynhoven, J. P. M. (2015b). Scaling Behavior of Dendritic Nanoparticle Mobility in Semidilute Polymer Solutions. Macromolecules, 48(20), 7585-7591. http://doi.org/10.1021/acs.macromol.5b01530

de Kort, D. W., van Duynhoven, J. P. M., Hoeben, F. J. M., Janssen, H. M., \& Van As, H. (2014). NMR nanoparticle diffusometry in hydrogels: enhancing sensitivity and selectivity. Analytical Chemistry, 86(18), 9229-9235. http://doi.org/10.1021/ac502211q

de Kort, D. W., van Duynhoven, J. P. M., Van As, H., \& Mariette, F. (2015c). Nanoparticle diffusometry for quantitative assessment of submicron structure in food biopolymer networks. Trends in Food Science of Technology, 42(1), 13-26. http://doi.org/10.1016/j.tifs.2014.11.003

Einhorn-Stoll, U., \& Drusch, S. (2015). Methods for investigation of diffusion processes and biopolymer physics in food gels. Current Opinion in Food Science, 3, 118-124. http://doi.org/10.1016/j.cofs.2015.07.002

Fitzpatrick, P., Meadows, J., Ratcliffe, I., \& Williams, P. A. (2013). Control of the properties of xanthan/glucomannan mixed gels by varying xanthan fine structure. Carbohydrate Polymers, 92(2), 1018-1025. http://doi.org/10.1016/j.carbpol.2012.10.049

Hadjiev, N. A., \& Amsden, B. G. (2015). An assessment of the ability of the obstruction-scaling model to estimate solute diffusion coefficients in hydrogels. Journal of Controlled Release, 199(C), 10-16. http://doi.org/10.1016/j.jconrel.2014.12.010

Hagman, J., Lorén, N., \& Hermansson, A.-M. (2010). Effect of Gelatin Gelation Kinetics on Probe Diffusion Determined by FRAP and Rheology. Biomacromolecules, 11(12), 3359-3366. http://doi.org/10.1021/bm1008487

Hagman, J., Lorén, N., \& Hermansson, A.-M. (2012). Probe diffusion in $\kappa$-carrageenan gels determined by fluorescence recovery after photobleaching. Food Hydrocolloids, 29(1), 106-115. http://doi.org/10.1016/j.foodhyd.2012.02.010

Haustein, E., \& Schwille, P. (2007). Fluorescence correlation spectroscopy: novel variations of an established technique. Annual Review of Biophysics and Biomolecular Structure, 36(1), 151-169. http://doi.org/10.1146/annurev.biophys.36.040306.132612

Hermansson, A. M., Eriksson, E., \& Jordansson, E. (1991). Effects of potassium, sodium and calcium on the microstructure and rheological behaviour of kappa-carrageenan gels. Carbohydrate Polymers, 16(3), 297-320. http://doi.org/10.1016/0144-8617(91)90115-S

Hohlbein, J., Gryte, K., Heilemann, M., \& Kapanidis, A. N. (2010). Surfing on a new wave of single-molecule fluorescence methods. Physical Biology, 7(3), 031001. http://doi.org/10.1088/1478$3975 / 7 / 3 / 031001$ 
Ikeda, S., Gohtani, S., Nishinari, K., \& Zhong, Q. (2012). Single Molecules and Networks of Xanthan Gum Probed by Atomic Force Microscopy. Food Science and Technology Research, 18(5), 741-745. http://doi.org/10.3136/fstr.18.741

Ikeda, S., Morris, V. J., \& Nishinari, K. (2001). Microstructure of aggregated and nonaggregated kappacarrageenan helices visualized by atomic force microscopy. Biomacromolecules, 2(4), 1331-1337. http://doi.org/10.1021/bm0156101

Johnson, E. M., Berk, D. A., Jain, R. K., \& Deen, W. M. (1996). Hindered diffusion in agarose gels: test of effective medium model. Biophysical Journal, 70(2), 1017-1023. http://doi.org/10.1016/S00063495(96)79645-5

Jonasson, J. K., Hagman, J., Lorén, N., Bernin, D., Nydén, M., \& Rudemo, M. (2010). Pixel-based analysis of FRAP data with a general initial bleaching profile. Journal of Microscopy, 239(2), 142-153. http://doi.org/10.1111/j.1365-2818.2009.03361.x

Jonasson, J. K., Lorén, N., Olofsson, P., Nydén, M., \& Rudemo, M. (2008). A pixel-based likelihood framework for analysis of fluorescence recovery after photobleaching data. Journal of Microscopy, 232(2), 260-269. http://doi.org/10.1111/j.1365-2818.2008.02097.x

Koynov, K., \& Butt, H.-J. (2012). Fluorescence correlation spectroscopy in colloid and interface science. Current Opinion in Colloid \& Interface Science, 17(6), 377-387. http://doi.org/10.1016/j.cocis.2012.09.003

Lopez-Sanchez, P., Schuster, E., Wang, D., Gidley, M. J., \& Strom, A. (2015). Diffusion of macromolecules in self-assembled cellulose/hemicellulose hydrogels. Soft Matter, 11(20), 4002-4010. http://doi.org/10.1039/C5SM00103J

Lorén, N., Nydén, M., \& Hermansson, A.-M. (2009a). Determination of local diffusion properties in heterogeneous biomaterials. Advances in Colloid and Interface Science, 150(1), 5-15. http://doi.org/10.1016/j.cis.2009.05.004

Lorén, N., Shtykova, L., Kidman, S., Jarvoll, P., Nydén, M., \& Hermansson, A.-M. (2009b). Dendrimer diffusion in kappa-carrageenan gel structures. Biomacromolecules, 10(2), 275-284. http://doi.org/10.1021/bm801013x

Mahmoudi, A. R., Shaban, E., Ghods, R., Jeddi-Tehrani, M., Emami, S., Rabbani, H., et al. (2011). Comparison of Photostability and Photobleaching Properties of FITC- and Dylight488- Conjugated Herceptin. International Journal of Green Nanotechnology, 3(4), 264-270. http://doi.org/10.1080/19430892.2011.633480

Masaro, L., \& Zhu, X. (1999). Physical models of diffusion for polymer solutions, gels and solids. Progress in Polymer Science, 24(5), 731-775. http://doi.org/10.1016/S0079-6700(99)00016-7

Mason, T. G., \& Weitz, D. A. (1995). Optical Measurements of Frequency-Dependent Linear Viscoelastic Moduli of Complex Fluids. Physical Review Letters, 74(7), 1250-1253. http://doi.org/10.1103/PhysRevLett.74.1250

Nolles, A., Westphal, A. H., de Hoop, J. A., Fokkink, R. G., Kleijn, J. M., van Berkel, W. J. H., \& Borst, J. W. (2015). Encapsulation of GFP in Complex Coacervate Core Micelles. Biomacromolecules, 16(5), 1542 1549. http://doi.org/10.1021/acs.biomac.5b00092

Salami, S., Rondeau-Mouro, C., Barhoum, M., van Duynhoven, J., \& Mariette, F. (2014). Translational and rotational diffusion of flexible PEG and rigid dendrimer probes in sodium caseinate dispersions and acid gels. Biopolymers, 101(9), 959-965. http://doi.org/10.1002/bip.22492

Salami, S., Rondeau-Mouro, C., van Duynhoven, J., \& Mariette, F. (2013). PFG-NMR self-diffusion in casein dispersions: Effects of probe size and protein aggregate size. Food Hydrocolloids, 31(2), 248-255. http://doi.org/10.1016/j.foodhyd.2012.10.020

Sato, T., Kojima, S., Norisuye, T., \& Fujita, H. (1984a). Double-Stranded Helix of Xanthan in Dilute Solution: Further Evidence. Polymer Journal, 16(5), 423-429. http://doi.org/10.1295/polymj.16.423

Sato, T., Norisuye, T., \& Fujita, H. (1984b). Double-stranded helix of xanthan: dimensional and 
hydrodynamic properties in $0.1 \mathrm{M}$ aqueous sodium chloride. Macromolecules, 17(12), 2696-2700. http://doi.org/10.1021/ma00142a043

Scholten, E., Moschakis, T., \& Biliaderis, C. G. (2014). Biopolymer composites for engineering food structures to control product functionality. Food Structure, 1(1), 39-54. http://doi.org/10.1016/j.foostr.2013.11.001

Schuster, E., Eckardt, J., Hermansson, A.-M., Larsson, A., Lorén, N., Altskär, A., \& Strom, A. (2014a). Microstructural, mechanical and mass transport properties of isotropic and capillary alginate gels. Soft Matter, 10(2), 357-366. http://doi.org/10.1039/C3SM52285G

Schuster, E., Hermansson, A.-M., Öhgren, C., Rudemo, M., \& Lorén, N. (2014b). Interactions and diffusion in fine-stranded $\beta$-lactoglobulin gels determined via FRAP and binding. Biophysical Journal, 106(1), 253-262. http://doi.org/10.1016/j.bpj.2013.11.2959

Seiffert, S., \& Oppermann, W. (2008). Diffusion of linear macromolecules and spherical particles in semidilute polymer solutions and polymer networks. Polymer, 49(19), 4115-4126. http://doi.org/10.1016/j.polymer.2008.07.036

Stejskal, E. O., \& Tanner, J. E. (1965). Spin diffusion measurements: Spin echoes in the presence of a timedependent field gradient. The Journal of Chemical Physics, 42, 288-292. http://doi.org/10.1063/1.1695690

Stokes, J. R. (2012). Food Biopolymer Gels, Microgel and Nanogel Structures, Formation and Rheology. In B. Bhandari \& Y. H. Roos (Eds.), Food Materials Science and Engineering (pp. 151-176). Oxford, UK: Wiley-Blackwell. http://doi.org/10.1002/9781118373903.ch6

Wassén, S., Bordes, R., Gebäck, T., Bernin, D., Schuster, E., Lorén, N., \& Hermansson, A.-M. (2014). Probe diffusion in phase-separated bicontinuous biopolymer gels. Soft Matter, 10(41), 8276-8287. http://doi.org/10.1039/c4sm01513d 



\title{
5
}

\section{Sub-micron structural heterogeneity in $\kappa$-carrageenan gels probed by nanoparticle diffusometry}

\begin{abstract}
A suite of spectroscopically active dendritic nanoparticles was used to probe structural heterogeneity in $\mathrm{Na}^{+} / \mathrm{K}^{+}$induced $\kappa$-carrageenan gels. Heterogeneity was apparent as nonGaussian self-diffusion of these nanoparticles as seen by ${ }^{1} \mathrm{H}$ NMR (PEGylated dendrimers, $d=7.5$ $\mathrm{nm}$ ), FRAP and RICS (PEGylated dendrimers with ATTO 488-labels, $d=6.0 \mathrm{~nm}$ ). The selfdiffusion coefficients of the slow nanoparticles were $\sim 10^{3}$ times lower than those of the fast particles on short time scales (up to hundreds of milliseconds), but the particles were fully immobilized on longer time scales, suggesting that the nanoparticles are trapped in nm-scale interstitials. The trapping effect allows a study of local water self-diffusion in the interstitials by ODNP-enhanced NMR spectroscopy. For this purpose, we used slightly larger TEMPO-labeled and PEGylated dendritic nanoparticles, which are partly immobilized by the network. This way we are able to demonstrate that heterogeneity in network density is also reflected in local water selfdiffusion; immobilized particles are in environment(s) where water diffusivity is significantly reduced.
\end{abstract}

Daan W. de Kort, Erich Schuster, Freek J.M. Hoeben, Henk M. Janssen, Niklas Lorén, Songi Han, Henk Van As, and John P.M. van Duynhoven, manuscript in preparation. 


\subsection{Introduction}

Biopolymer hydrogels comprise crosslinked, percolating polymer networks, giving rise to a tortuous network of water-filled interstitials. Microstructural characterization of such networks by optical or electron microscopy can be problematic because they require invasive sample preparation steps that alter the structure of the network (de Kort, van Duynhoven, Van As, \& Mariette, 2015c). Alternatively, information about microstructure can be obtained from the selfdiffusion of embedded nanoparticles with sizes on the order of the size of the interstitials. If nanoparticles that are dissolved in the continuous phase of a polymer gel are smaller than the structural features of the gel, their mobility will be determined by "local" properties very different from the macroscopic ones. If the size of particles is smaller than, but on the order of, the distance between the polymer strands or fibers, the particles are still free to diffuse in the continuous phase but will be influenced by the presence of the polymers. For this reason, the self-diffusion coefficient of nanoparticles diffusing in biopolymer solutions and gels is typically significantly lower that the self-diffusion coefficient in water (although much higher than expected based on the macroscopic zero-shear viscosity) (Bourouina et al., 2015; de Kort, Rombouts, Hoeben, Janssen, Van As, et al., 2015b; de Kort, van Duynhoven, Van As, \& Mariette, 2015c). Many models exist that relate these reduced self-diffusion coefficients with the polymer concentration (volume fraction), polymer strand thickness, nanoparticle diameter and network "mesh size" (de Kort, van Duynhoven, Van As, \& Mariette, 2015c; Masaro \& Zhu, 1999). The various models exploit different physical phenomena to explain the origin of the reduced diffusion coefficient: "Obstruction effect"-type models imply that the rigid polymer network is tortuous and imposes an increased path length for particles moving between two points in the network. Other models provide scaling laws for the hydrodynamic friction of the nanoparticles and the polymer chains. However, such models predict long-term, average diffusion coefficients (assuming the shape of the Van Hove self-correlation function is Gaussian) but do not explicitly consider the effect of microstructural heterogeneity on the diffusion coefficients of solutes at shorter time scales. Lorén et al. have shown for the first time non-Gaussian diffusion of dendrimer nanoparticles in $\kappa$ carrageenan gels on the $\sim 10^{1}-10^{2} \mathrm{~ms}$ time scale (Lorén et al., 2009b). In this work, we will exploit this phenomenon to demonstrate nanometer-scale microstructural heterogeneity in such gels.

$\kappa$-Carrageenan is a linear polysaccharide that is used industrially as a gelling agent. Gelation of $\kappa$ carrageenan occurs upon cooling a warm aqueous solution, during which the polymer coils first form helices that subsequently aggregate in a side-by-side manner (Hermansson, 1989; Viebke, Piculell, \& Nilsson, 1994). The coil-to-helix transition, which is essential for eventual gelation, is very sensitive for binding of cations such as potassium, calcium, or sodium ions, strongly influencing the microstructure and elastic strength of the gels (Hermansson, Eriksson, \& Jordansson, 1991). The microstructure of $\kappa$-carrageenan gels can therefore essentially be controlled by the choice of cations.

In previous work on nanoparticle diffusion in heterogeneous $\kappa$-carrageenan gels (Bourouina et al., 
2015; de Kort, van Duynhoven, Hoeben, Janssen, \& Van As, 2014), we have shown that, on the millisecond time scale, the mobility of a minor fraction $(<10 \%)$ of nonsticky nanoparticles (sizes between 5 and $20 \mathrm{~nm}$ ) is reduced by at least an order of magnitude. The degree of immobilization was shown to increase with polymer concentration and particle size. At the same time, the diffusion coefficient of the more mobile fraction decreased with polymer concentration. This decrease could be described accurately with a diffusion model that yielded an average polymer strand thickness of $\sim 7 \mathrm{~nm}$, in line with side-by-side stacking of polymer helices. We rationalized this behavior by considering the biopolymer network as a heterogeneous "sieve", through which the particles have to pass when moving between two points in the system (Lorén, Nydén, \& Hermansson, 2009a). With increasing polymer concentration, particles will experience stronger sieving. Stronger sieving will also occur with increasing particle size. One would expect, however, that sieving will only affect particle mobility on intermediate time scales and that a single diffusion coefficient will be observed on longer time scales when the sieving effect has averaged out. However, by using pulsed-field gradient (PFG) NMR diffusometry with various observation times, we did not observe any time averaging of the two fractions, neither did we observe diffusive exchange between the fractions on the $10^{2} \mathrm{~ms}$ time scale (de Kort, Hoeben, Janssen, Bourouina, Kleijn, et al., 2015a).

In this paper, we further investigate the heterogeneity in $\kappa$-carrageenan gels as apparent from the bimodal diffusion of nanoparticles. Herein we use gels at low $\mathrm{Na}^{+}$concentrations that are known to be particularly heterogeneous. This allows a more precise study of bimodal diffusion for which we use PFG NMR diffusometry and raster image correlation spectroscopy (RICS) to show that the slow-diffusing particle fraction is three orders of magnitude slower than the fast-diffusing particle fraction. We conjecture that the particles are trapped in small cavities from which they cannot escape. This would explain the lack of diffusive exchange between the two fractions on the millisecond time scale.

We then exploit the entrapment effect to study water dynamics within the polymer gels. Labeling dendritic nanoparticles with nitroxide-based paramagnetic TEMPO spin labels allows us to use Overhauser dynamic nuclear polarization (ODNP)-enhanced NMR to probe the water dynamics in the immediate vicinity $(0.5-1.5 \mathrm{~nm})$ of the nanoparticles (Armstrong \& Han, 2009; Franck, Pavlova, Scott, \& Han, 2013). We examine the local water dynamics of a system where both the mobile and trapped particle fractions are present and compare the results to the dynamics in a system where the mobile particles have been washed away by keeping the gels in a much larger volume of salt solution for multiple days. This way we are able to observe differences in local water mobility close to the polymer strands.

\subsection{Materials and Methods}

Sample preparation. $\kappa$-Carrageenan gels were prepared by suspending $\kappa$-carrageenan powder ( 2 $\mathrm{wt} \%$ ) in a solution of sodium chloride (between 0-200 mM), potassium chloride (20 mM), and 
labeled dendritic nanoparticles in water $(0.1 \mathrm{wt} \%)$, followed by heating to $80{ }^{\circ} \mathrm{C}$ for 15 minutes. This allows $\kappa$-carrageenan to dissolve. The solutions are subsequently allowed to cool down to room temperature during which gelation (together with the nanoparticles) takes place. "Washed" gels were prepared by keeping a $\sim 3 \times 3 \times 3 \mathrm{~mm}$ gel cube in a $\sim 50 \mathrm{x}$ larger volume of corresponding salt solution for a week and refreshing the medium daily.

Labeled dendritic nanoparticles. Besides ${ }^{19}$ F-labeled PEGylated generation-1, -3 and -5 (G1, G3, G5) poly(propylene imine) dendritic nanoparticles as presented in our previous work (de Kort et al., 2014), we used additional labeled analogs (SyMO-Chem B.V., Eindhoven, Netherlands). These particles again had a core based on a G5 dendrimer and a brush-like polyethylene glycol (PEG) corona. Labels had been introduced beneath the PEG corona. One particle contained ATTO-488 fluorescent dyes at the interface between core and corona, while the other contained TEMPO spin labels and a spacer to slightly increase their size. Synthetic procedures are included in the Supporting Information, section S5.1, and cartoons of the three particles used in this work are presented in Figure 5.1.

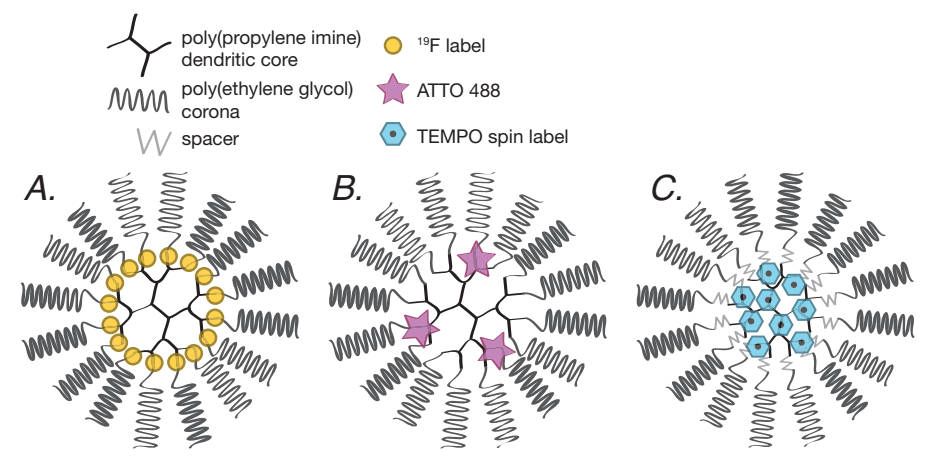

Figure 5.1: Schematic illustrations of nanoparticles with G5 PPI dendrimer cores used in this study (these illustrations do not necessarily reflect the true conformation of the particles in solution): $(A){ }^{19} \mathrm{~F}$ labeled, PEGylated dendrimers $\left(d=7.5 \pm 0.3 \mathrm{~nm}\right.$, cf. Table S6.1) that can be observed by ${ }^{19} \mathrm{~F}{ }^{1} \mathrm{H}$ NMR via the signal of the PEG corona, $(B)$ PEGylated ATTO-488-labelefgd dendrimers $(d \sim 6.0 \pm 0.8 \mathrm{~nm}$ as determined by FCS) that can be observed by FRAP and RICS and (C) PEGylated TEMPO-labeled dendrimers for Overhauser DNP-enhanced NMR spectroscopy, with a spacer to slightly increase their diameter ( $d=9.9 \pm 0.2 \mathrm{~nm}$ as determined by NMR diffusometry). The diameter increase aims to increase the fraction of immobilized particles (see Results section).

${ }^{1} H$ PFG NMR diffusometry. Because of its higher sensitivity, diffusion-ordered spectroscopy (DOSY) measurements were carried out on ${ }^{1} \mathrm{H}$ instead of ${ }^{19} \mathrm{~F}$. In this work, ${ }^{19} \mathrm{~F}$ measurements were only used as a cross-check to verify the absence of the effects of background signal in the ${ }^{1} \mathrm{H}$ measurements. ${ }^{1} \mathrm{H}$ DOSY was performed with a Bruker Avance II spectrometer at $300 \mathrm{MHz}$ 
resonance frequency for ${ }^{1} \mathrm{H}$, equipped with a Bruker diff25 gradient probe (maximum pulsed field gradient intensity $9.60 \mathrm{~T} / \mathrm{m}$ ). The probe was equipped with a $10-\mathrm{mm}$ RF insert tuned to the ${ }^{1} \mathrm{H}$ resonance frequency. Experiments and data analysis were performed as outlined in (de Kort, Rombouts, Hoeben, Janssen, Van As, et al., 2015b). In short, DOSY experiments based on a stimulated echo were performed by stepwise variation of the gradient pulse amplitude at an effective gradient pulse duration of $5 \mathrm{~ms}$, while keeping the diffusion-observation time at $200 \mathrm{~ms}$. The minimum gradient amplitude was chosen to be high enough to attenuate the ${ }^{1} \mathrm{H}$ signal of water almost completely. The attenuation of the ${ }^{1} \mathrm{H}$ echo intensity of the PEG corona as a function of increasing gradient amplitude is described by a sum of Stejskal-Tanner-type exponentials $\left(I / I_{0}\right)=A e^{-b D}$ and $b=(\gamma \delta g)^{2}(\Delta-\delta / 3)$, where $\left(I / I_{0}\right)$ is the signal attenuation, $D$ the diffusion coefficient, $\gamma$ the gyromagnetic ratio, $\delta$ the width of the gradient pulse, $g$ the gradient amplitude and $\Delta$ the diffusion-observation time (Stejskal \& Tanner, 1965).

RICS. Raster image correlation spectroscopy (Digman et al., 2005) was carried out on washed gels only. A Leica SP5 AOBS setup was used with a $63 \times, 1.2$ NA water objective using the following settings: $512 \times 512$ pixels, zoom factor 10 , and scan rate $10 \mathrm{~Hz}$, yielding a pixel size of $0.0482 \mu \mathrm{m}$ and a pixel dwell time $(t \mathrm{P})$ of $0.48 \mathrm{~ms}$. A 488-nm Ar-laser was used to excite the ATTO-488 labeled dendritic nanoparticles. The laser power on the stage was $10 \mu \mathrm{W}$ during the experiments. The recorded RICS data was analyzed to yield one diffusion coefficient per $512 \times 512$ pixel data set. Furthermore, ca. $70 \times 70$ pixel sized regions of interest were analyzed separately, in order to segment the data and estimate local diffusion coefficients.

EPR and Overhauser DNP-enhanced NMR spectroscopy. Cw-EPR spectra were measured on a Bruker EMX X-band spectrometer equipped with a cylindrical (TE011) cavity. Samples were irradiated at $9.8 \mathrm{GHz}$ with the center field set at $3480 \mathrm{G}$ and sweep width of $150 \mathrm{G}$. The field modulation amplitude was kept below 0.2 times the center peak line width to acquire the intrinsic EPR lineshapes and amplitudes. Nitrogen gas was streamed through the cavity at $14 \mathrm{~L} / \mathrm{min}$, and all spectra were acquired at $298 \mathrm{~K}$.

Local water diffusion coefficients within $0.5-1.5 \mathrm{~nm}$ of nitroxide radical-based TEMPO spin labels were measured by Overhauser DNP-enhanced NMR spectroscopy. The same samples and instrument were used as described for X-band EPR experiments. The magnetic field and frequency for irradiation were set to the center resonance of the nitroxide EPR spectra. Samples were positioned in a home-built U-shaped NMR coil ( $\mathrm{Cu}$ wire, $28 \mathrm{AWG}$ ) tuned to $14.8 \mathrm{MHz}$ and connected to a broadband channel of a Bruker Avance NMR spectrometer, as described in detail elsewhere (Armstrong \& Han, 2007; Ortony et al., 2011). Longitudinal relaxation time of water ${ }^{1} \mathrm{H}$, in the presence $\left(T_{1}\right)$ and absence $\left(T_{1,0}\right)$ of the nitroxide spin labels, was carefully measured using an inversion recovery pulse sequence. The ${ }^{1} \mathrm{H}$ NMR signal enhancement of water was recorded as a function of increasing microwave power that was varied using a home-built X-band 
microwave amplifier with a power output between $0.1 \mathrm{~mW}$ and $1.5 \mathrm{~W} .{ }^{1} \mathrm{H}$ NMR spectra were integrated over their absorption peak and the absolute values were plotted versus input microwave power. As detailed in (Franck et al., 2013), from the NMR signal enhancement extrapolated to infinitely high microwave power $\left(E_{\max }\right)$, together with $T_{1}$ and $T_{1,0}$, the key DNP parameter termed the coupling factor was computed, which corresponds to the dipolar electron- ${ }^{1} \mathrm{H}$ cross relaxation efficiency with respect to all ${ }^{1} \mathrm{H}$ relaxation processes. From the DNP coupling factor, using the appropriate molecular dynamics model and spectral densities, the dipolar correlation time for translational diffusion dynamics of the local water in the dipolar coupling vicinity of the spin label, was determined. From the dipolar correlation time, $\tau$, the water diffusion coefficient, $D$, was determined from the relation, $\tau=\left(d^{2} / D\right)$, where $d=3.0 \AA$ represents the distance of the closest approach of the water to the radical electron spin label (Armstrong \& Han, 2007; 2009). Error bars on the DNP measurements are approximated to fall between 3 and 5\%, as estimated from the quality of $E_{\max }$ and $T_{1}$ fitting curves.

\subsection{Results and Discussion}

PFG NMR diffusometry. In previous work, we have shown that self-diffusion coefficients of nonsticky G1, G3 and G5 dendritic ${ }^{19}$ F-labeled nanoparticles with diameters of 3.4-7.5 nm, as determined by PFG NMR, decrease with $\kappa$-carrageenan concentration (de Kort et al., 2014). In the current work, we carried out new PFG NMR experiments using the same PEGylated G1, G3 and G5 nanoparticles in gels that were induced at lower sodium concentrations $\left(\leq 200 \mathrm{mM} \mathrm{Na}{ }^{+}\right)$, which is known to lead to more heterogeneous network structures (Lorén et al., 2009b). We use ${ }^{1} \mathrm{H}$ NMR because it provides a higher signal intensity, while we can filter out the signal of the rigid $\kappa$-carrageenan matrix due to its short $T_{2}$. For these experiments we used a single carrageenan concentration of $2 \mathrm{wt} \%$ at a $\mathrm{K}^{+}$concentration of $20 \mathrm{mM}$. The resulting PFG NMR attenuation curves are shown in Figure 5.2.

At lower $\mathrm{Na}^{+}$concentrations, we see a significant amplitude offset at higher $b$-values that is hardly visible at $200 \mathrm{mM} \mathrm{Na}^{+}$as used in the previous experiments (de Kort et al., 2014). The sum of two exponentials was fitted to describe the shape of the attenuation curves. This appears, however, not an optimal fit for the $0 \mathrm{mM} \mathrm{Na}{ }^{+}$-curve, suggesting that the "slow" particle fraction component describes a distribution of low diffusion coefficients. In fact, the offset is not a flat contribution, but slightly decreases as a function of $b$, indicating that the slower particles are not completely immobilized. Diffusion coefficients and relative amplitudes obtained from the two-component fit are shown in Figure 5.3. 


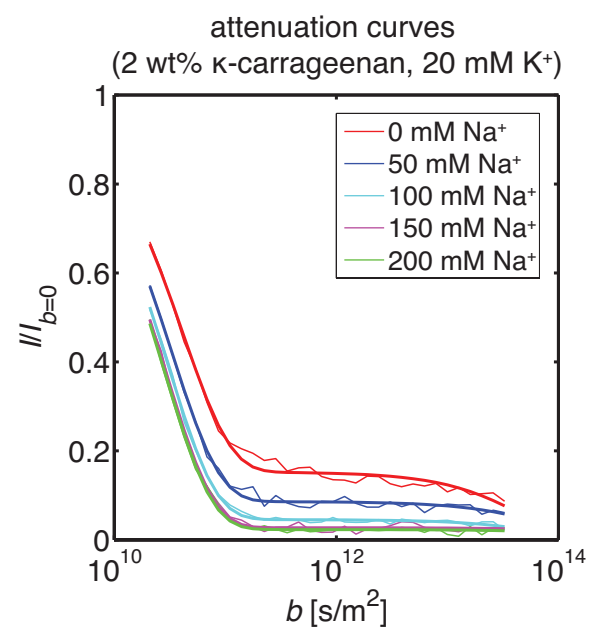

Figure 5.2: Plots of PFG $\left({ }^{1} \mathrm{H}\right)$ NMR signal attenuation $\left(I / I_{b=0}\right)$ as a function of $b$ for G5 $(d=7.5 \mathrm{~nm})$ dendritic nanoparticles in $2 \mathrm{wt} \% \mathrm{\kappa}$-carrageenan gels induced at different $\mathrm{Na}^{+}$and constant $20 \mathrm{mM} \mathrm{K}$ concentrations. The initial part of the curves is missing because a nonzero initial gradient amplitude was used to suppress the ${ }^{1} \mathrm{H}$ NMR signal of water and low-molecular-weight solutes. The curves have been normalized to the back-predicted amplitude at zero gradient amplitude. The curves have been fitted with a sum of two Stejskal-Tanner exponentials $\left(I / I_{b=0}\right)=\Sigma_{i} A_{i} e^{-b D_{i}}$.
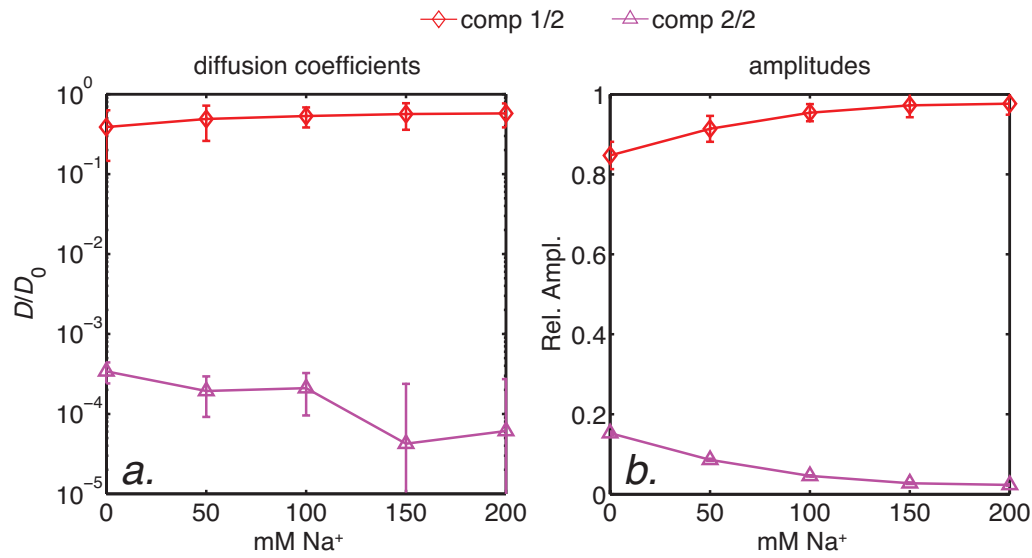

Figure 5.3: (a) Self-diffusion coefficients of G5 $(d=7.5 \mathrm{~nm})$ dendritic nanoparticles in $2 \mathrm{wt} \% \mathrm{k}$ carrageenan gels induced at different $\mathrm{Na}^{+}$concentrations and constant $20 \mathrm{mM} \mathrm{K}{ }^{+}$concentration (normalized to the diffusion coefficient in water, $5 \times 10^{-11} \mathrm{~m}^{2} / \mathrm{s}$ ) and $(\boldsymbol{b})$ relative amplitudes of both components of the two-component fit (goodness of fit in SI, section S5.2). No slow-diffusing fraction was observed for the smaller G1 $(d=3.4)$ and G3 $(4.6 \mathrm{~nm})$ dendritic particles with a similar non-sticky design (cf. SI, section S5.3). 
While the amplitudes show that the fraction of slow particles decreases significantly with salt concentration, the plot of diffusion coefficients shows that the slower fraction has diffusion coefficients more than three orders of magnitude lower $\left(\sim 10^{-14} \mathrm{~m}^{2} / \mathrm{s}\right)$ than the faster fraction $\left(\sim 10^{-11} \mathrm{~m}^{2} / \mathrm{s}\right)$. In the $200 \mathrm{~ms}$ experimental time window, the root mean square displacement $(2 D t)^{1 / 2}$ of the faster fraction is $>5 \mu \mathrm{m}$, which is beyond the size of microstructural features typically observed in these gels. The higher diffusion coefficients are thus effectively long-time diffusion coefficients as predicted from diffusion models. The root mean square displacement of the slower fraction over the course of $200 \mathrm{~ms}$ is in the tens-of-nm range. It is possible that this reflects either the size of cavities from which the particles cannot escape, or the presence of denser domains with sizes up to hundreds of nanometers, from which they will escape at only longer time scales that are not accessible by PFG NMR diffusometry. To test this, we kept gel cubes (approximately $3 \times 3 \times 3 \mathrm{~mm}$ ) in a much larger volume of salt solution and waited for a week, while daily refreshing the salt medium, to allow all non-trapped particles to escape from the gel. Upon washing the faster component could not be observed anymore in the PFG attenuation curve, and only the slowly diffusing component could be observed. For these experiments we used fluorescent (ATTO-488-labeled) nanoparticles of the same design and size to allow for confocallaser-scanning-microscopy (CLSM)-based fluorescence measurements that also allow us to localize the trapped particles.

FRAP and RICS. In the gel series loaded with fluorescent ATTO-488-labeled nanoparticles, we observe via CLSM diffusion measurements two discrete populations that diffuse with two discrete diffusion coefficients, in agreement with the findings by PFG NMR diffusometry. By FRAP, after a bleaching pulse, we see that the fluorescence recovers, but only partly. Figure 5.4a shows a representative fluorescence recovery curve, indicating a very low diffusion coefficient for the slower fraction, which is however difficult to quantify from FRAP due to background bleaching effects. We used a complimentary fluorescence method, RICS, to determine the diffusion coefficient of the slow fraction, for which we use the remaining fluorescence in the washed gels.

Furthermore, the segmentation of RICS images and the analysis of diffusion coefficients in selected ROIs, as shown in Figure 5.5, demonstrates that the slow component originates from brighter spots in the images. Analysis of the dark/purple region yields regions without significant signal and thus without any apparent remaining mobile particles. The bright regions however are diffusion probes (or possibly aggregated probes, although the size of the fluorescence spot does not necessarily correspond to the size of the particles, which is below the diffraction limit, and the number of ATTO-488 molecules per nanoparticle can vary), for which we still observe a low degree in mobility in very good agreement with the PFG NMR results. Because the RICS experiments were performed after the washing step that should have allowed even non-trapped particles located in small, but high-density domains to leave the gel, we conclude that the "slow fraction" of particles is indeed trapped, but still slightly mobile. From the CLSM images we cannot 
be certain about the manner in which the particles are trapped. This might be inside a small (max $\sim 100 \mathrm{~nm}$ ) interstitial that still allows for some mobility of the particle, or that the particles are somehow immobilized on the polymer strands themselves and that the particle and polymer strands themselves still have a small degree of mobility within the gel structure. The fact that the particles are either trapped in small interstitials, or otherwise closer to the polymer strands than the fast fraction of particles freely diffusing in the water phase, gives us a unique opportunity to utilize the nanoparticles for a study of local water dynamics in these gels.
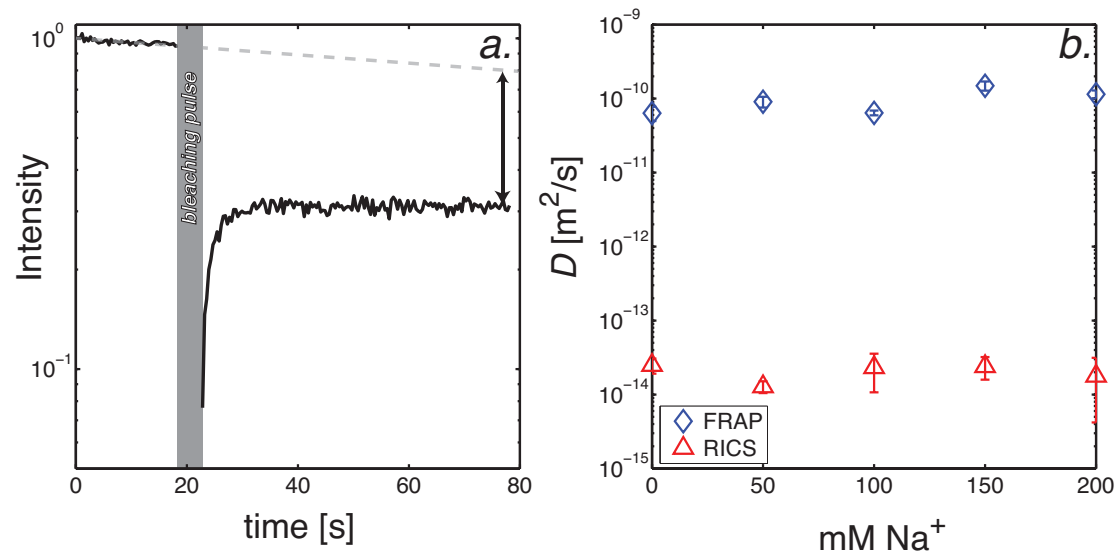

Figure 5.4: $(\boldsymbol{a})$ Example FRAP curve (G5-ATTO-488 nanoparticle in a $k$-carrageenan gels prepared with $20 \mathrm{mM} \mathrm{K}^{+}$and $0 \mathrm{mM} \mathrm{Na}^{+}$) showing initial recovery of fluorescence after bleaching, after which the recovery levels off. The pre-bleach part of the curve shows gradual decay of fluorescence over the course of the measurement, because of which it is problematic to reliably determine a diffusion coefficient for the slow fraction. $(\boldsymbol{b})$ Diffusion coefficients of G5-ATTO-488 $(d=7.5 \mathrm{~nm})$ particles as determined by CLSM. The fast diffusion coefficient is determined by FRAP, where only the first 10 post bleach images were analyzed. Via this caveat the slow fraction can be disregarded during data analysis. The diffusion coefficient of the slow fraction is determined by RICS on washed gels.

Local mobility of water probed by Overhauser DNP-enhanced NMR spectroscopy. To measure water mobility in the direct vicinity of the nanoparticles, we functionalized them with TEMPO spin labels, and added a spacer to slightly increase the diameter and hence the fraction of particles that become immobilized. We expected that the immobilized dendrimers indeed probe a higher degree of water retardation than the ensemble of immobilized and mobile particles, because immobilized particles must be closer to the $\kappa$-carrageenan strands and thus to the (retarded) hydration water surrounding the strands. Hence we washed away the mobile particles in the same manner as described above for the RICS experiments. We measured local water diffusion in these samples (Figure 5.6) and EPR spectra of the samples before and after the washing step (Figure 5.7). 

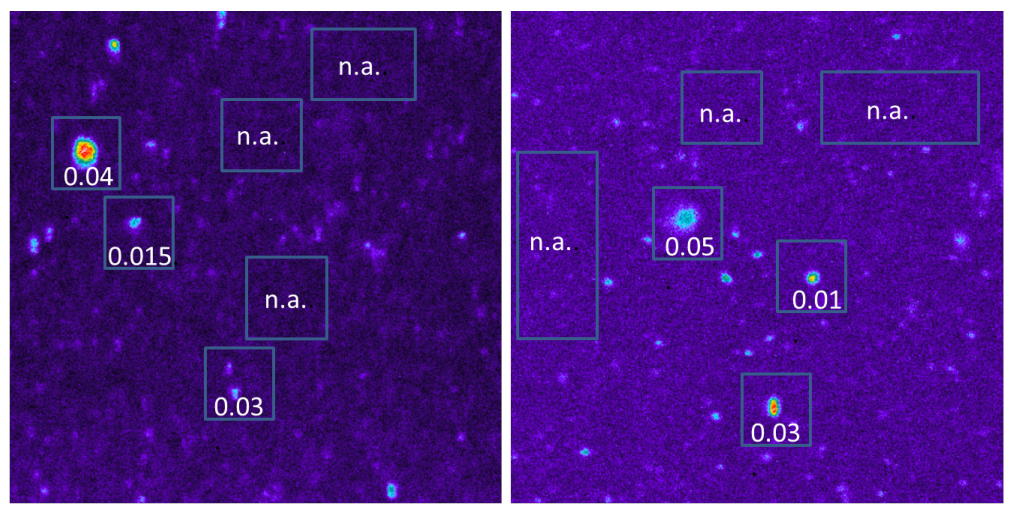

Figure 5.5: Localized estimation of diffusion coefficients of G5-ATTO-488 nanoparticle in $\kappa$ carrageenan gels prepared with $20 \mathrm{mM} \mathrm{K}^{+}$and respectively 0 and $200 \mathrm{mM} \mathrm{Na}^{+}$via RICS. (left) $0 \mathrm{mM}$ $\mathrm{Na}^{+}, D_{\text {avg }}=2 \times 10^{-14} \mathrm{~m}^{2} / \mathrm{s}$ (averaged over the whole image). (right) $200 \mathrm{mM} \mathrm{Na}{ }^{+}, D_{\text {avg }}=3 \times 10^{-14} \mathrm{~m}^{2} / \mathrm{s}$. The field of view is $24.7 \mu \mathrm{m}$.

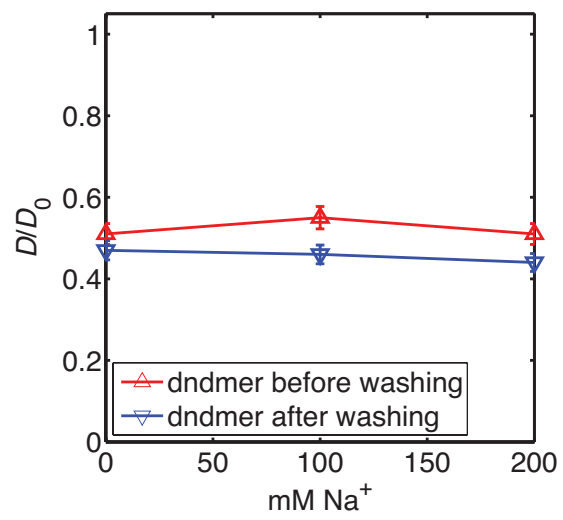

Figure 5.6: Retardation of local water self-diffusion in $2 \% \kappa$-carrageenan gels as observed by ODNPenhanced NMR spectroscopy. $D_{0}$ is the water diffusion coefficient as determined by this method in water without polymer. Experiments were performed in $2 \mathrm{wt} \% \mathrm{k}$-carrageenan, $20 \mathrm{mM} \mathrm{K}{ }^{+}$gels. After the washing step, the local water diffusion coefficient probed by the remaining dendrimers is significantly lower than before the washing step. 


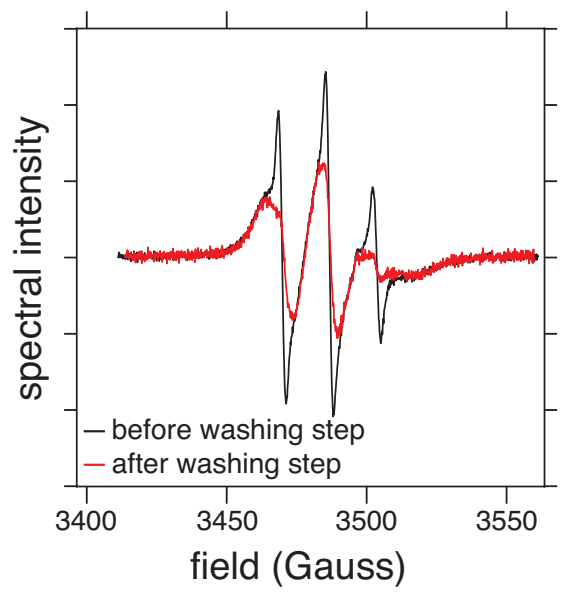

Figure 5.7: $\mathrm{Cw}$-EPR spectra of TEMPO-functionalized dendrimers in $2 \mathrm{wt} \% \mathrm{k}$-carrageenan (this example: $0 \mathrm{mM} \mathrm{Na}^{+} / 20 \mathrm{mM} \mathrm{K}^{+}$) before and after the washing step show that after washing, only the broad component of the spectrum remains (spectral double integral ratio after/before washing $=0.57$, showing significant immobilization), demonstrating the permanently reduced mobility of the slower particle fraction.

In Figure 5.7, in the cw-EPR spectrum of TEMPO-dendrimers before the washing step, both a broad and narrow component can be seen. Upon washing away the mobile particle fraction, all narrow features disappear from the spectrum while the broad features remain. This directly reflects the decreased mobility of the remaining particles. The difference between the double integral of both spectra shows that approximately $57 \%$ of particles are immobilized. This fraction is higher than determined in the PFG NMR experiments, but on the other hand the TEMPOfunctionalized dendrimers are slightly larger, thus provoking more immobilization if this is indeed a topological effect.

As can be seen in Figure 5.6, the washing step that removes the mobile dendrimers also leads to lowering of the apparent local water diffusion coefficient, which is in agreement with the idea that the immobilized particles are located in closer vicinity to the polymer strands and are therefore surrounded by more viscous hydration water. Besides this, we do not see any significant effect of $\mathrm{Na}^{+}$concentration of the apparent local water mobility, neither before nor after the washing step. This is not surprising after the washing step, since the remaining (immobile) particles will be in similar environments that lead to their immobilization in the first place. Before the washing step, however, where we expect the apparent local water diffusivity to be an average of the diffusivity around the mobile and immobile particles, the observed diffusion coefficients are the same within the experimental error for all $\mathrm{Na}^{+}$concentrations, and hence do not correlate with the observed differences in the magnitude of the immobilized fraction between the three different $\mathrm{Na}^{+}-$ concentrations. This can only be the case if the immobilized particles have a dominating effect on 
the efficiency of the EPR saturation from which the diffusion coefficient is calculated. This can be understood from the non-linear relation between the coupling factor and the diffusion coefficient, which can be calculated through Hwang and Freed's expression for the spectral density function (Franck et al., 2013). This expression predicts a significantly lower diffusion coefficient for a marginally decreasing coupling factor that is already low (as in our case, where the coupling factor for the immobilized particles is $\sim 0.015$ ).

\subsection{Conclusions}

Network heterogeneity in $\mathrm{Na}^{+} / \mathrm{K}^{+}$-induced $\kappa$-carrageenan gels could be observed as bimodal selfdiffusion of dendritic nanoparticles $(d=7.5 \mathrm{~nm})$, the fraction of slow-diffusing particles decreased with $\mathrm{Na}^{+}$concentration. The slow particles diffused $\sim 10^{3}$ times slower than the fast particles on short time scales (up to hundreds of milliseconds). These "slow" particles were immobilized on longer time scales, indicating they were trapped in small interstitials. The number of immobilized particles decreases with $\mathrm{Na}^{+}$-concentration, in line with reduced structural heterogeneity as observed in earlier work (Lorén et al., 2009b). The trapping effect allowed a study of water mobility in the $\kappa$-carrageenan network interstitials by ODNP-enhanced NMR measurements on TEMPO-labeled dendritic nanoparticles, which are partly immobilized within the network. The water diffusivity surrounding the immobilized particles is significantly retarded compared to the average water diffusivity apparent from the gels in which mobile particles were still present. This demonstrates that the immobilized particles are in a confined environment with reduced local water diffusivity.

\section{Supporting Information}

Supporting information is included below: S5.1. Synthetic procedures for TEMPO- and ATTOlabeled dendrimers. S5.2. Exponential fitting of G5-dendrimer ${ }^{1} \mathrm{H}$ NMR attenuation curves: goodness of fit. S5.3. ${ }^{1} \mathrm{H}$ NMR attenuation curves for G1 and G3 dendrimers in $2 \mathrm{wt} \% \mathrm{\kappa}$ carrageenan.

\section{Acknowledgments}

Lonneke Zuidgeest (Unilever R\&D, Vlaardingen, Netherlands), Meike Emondts, Ryan Barnes, Jinsuk Song, and Kuo-Ying Huang (University of California, Santa Barbara) are acknowledged for their contributions to this work. 


\section{S5.1 Synthesis of TEMPO- and ATTO-labeled dendrimers}

\section{S5.1.1 Synthesis of TEMPO-labeled dendrimers}

Materials. All reagents, chemicals, materials and solvents were obtained from commercial sources, and were used as received: Biosolve, Merck and Cambridge Isotope Laboratories for (deuterated) solvents; Aldrich, Acros, ABCR, Merck and Fluka for chemicals, materials and reagents. All solvents were of AR quality. Moisture or oxygen-sensitive reactions were performed under an Ar atmosphere. Hygroscopic compounds (e.g. containing ethylene oxide chains) were stored under vacuum in a desiccator over $\mathrm{P}_{2} \mathrm{O}_{5}$. Bio-Beads S-X1 was obtained from Bio-Rad Laboratories. ATTO488-NHS ester was purchased from ATTO-TEC GmbH and used as received (the structure of the anion is unknown). mPEG750-COOTFP (de Kort, Rombouts, Hoeben, Janssen, Van As, et al., 2015b) and 3,6,9,12,15,18,21,24,27,30,33-undecaoxatetratriacontanoic acid (Hattori, Okada, Kondoh, \& Tsukaguchi, 2009) were prepared according to literature procedures.

Methods. ${ }^{1} \mathrm{H}-\mathrm{NMR},{ }^{13} \mathrm{C}-\mathrm{NMR}$ and ${ }^{19} \mathrm{~F}-\mathrm{NMR}$ spectra were recorded on a Varian Mercury (400 $\mathrm{MHz}$ for ${ }^{1} \mathrm{H}-\mathrm{NMR}, 100 \mathrm{MHz}$ for ${ }^{13} \mathrm{C}-\mathrm{NMR}$ and $376 \mathrm{MHz}$ for $\left.{ }^{19} \mathrm{~F}-\mathrm{NMR}\right)$ spectrometer at $298 \mathrm{~K}$. Chemical shifts are reported in ppm downfield from TMS at r.t. using deuterated chloroform $\left(\mathrm{CDCl}_{3}\right)$ as a solvent and internal standard unless otherwise indicated. Abbreviations used for splitting patterns are $\mathrm{s}=$ singlet, $\mathrm{t}=$ triplet, $\mathrm{q}=$ quartet, $\mathrm{m}=$ multiplet and $\mathrm{br}=$ broad. $\mathrm{IR}$ spectra were recorded on a Perkin Elmer 1600 FT-IR (UATR). Preparative size exclusion chromatography was performed using Bio-Rad Bio-Beads S-X1 (200-400 mesh). MALDI-TOF MS was measured on a Bruker Autoflex Speed. LC-MS was performed using a Shimadzu LC-10 AD VP series HPLC coupled to a diode array detector (Finnigan Surveyor PDA Plus detector, Thermo Electron Corporation) and an Ion-Trap (LCQFleet, Thermo Scientific) where ions were created via electrospray ionization (ESI). Analyses were performed using a Alltech Alltima HP $\mathrm{C}_{18}$ $3 \mu$ column using an injection volume of $1-4 \mu \mathrm{L}$, a flow rate of $0.2 \mathrm{~mL} \mathrm{~min}^{-1}$ and typically a gradient ( $5 \%$ to $100 \%$ in $10 \mathrm{~min}$, held at $100 \%$ for a further $3 \mathrm{~min}$ ) of $\mathrm{CH}_{3} \mathrm{CN}$ in $\mathrm{H}_{2} \mathrm{O}$ (both containing $0.1 \%$ formic acid) at $298 \mathrm{~K}$. 


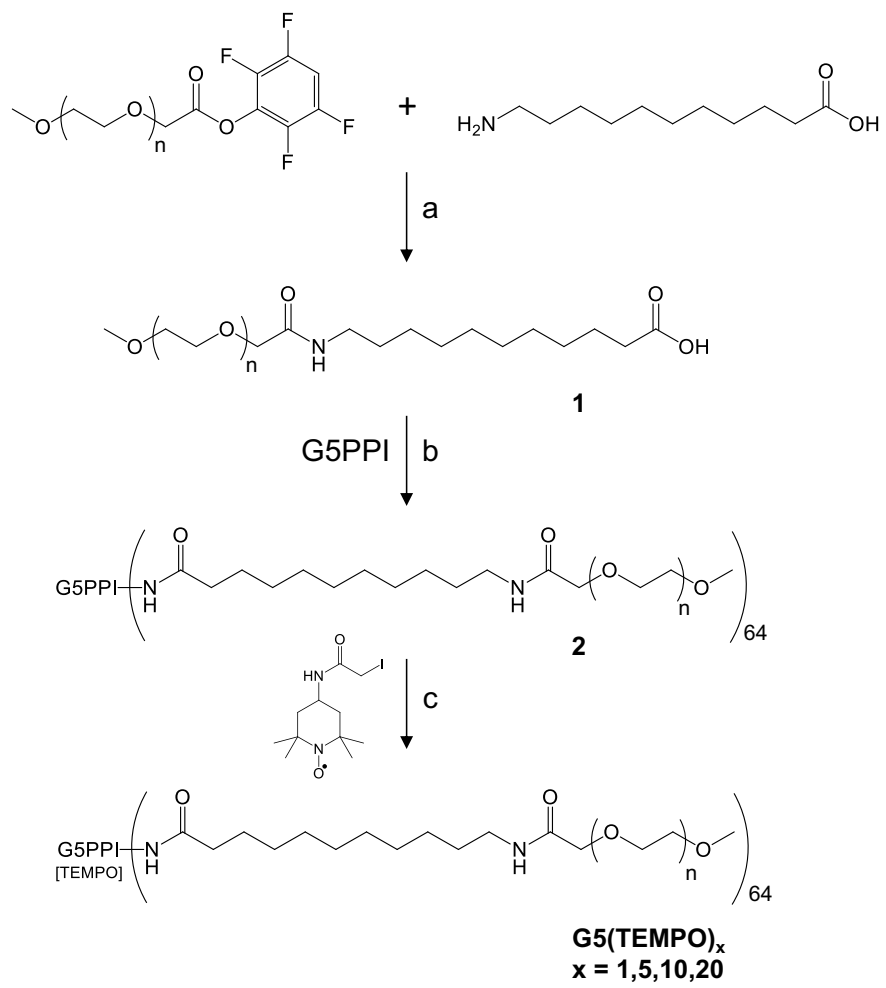

Scheme S5.1: Synthesis of generation 5 dendritic nanoparticles with an mPEG750 coating (distribution around $\mathrm{n}=16$ ) and varying equivalents of bound TEMPO (a) N,Ndiisopropylethylamine, dioxane / $\mathrm{H}_{2} \mathrm{O} \quad 1: 1,1$ h, r.t., $66 \%$ (b) $\operatorname{G5PPI}\left(\mathrm{NH}_{2}\right)_{64}, \quad \mathrm{~N}, \mathrm{~N}-$ diisopropylethylamine, PyBOP, $\mathrm{CH}_{2} \mathrm{Cl}_{2}, 1$ h, r.t., $84 \%$ (c) 4-((2-iodoacetyl)amino)-2,2,6,6tetramethyl-1-piperidinyloxy, methanol, $66 \mathrm{~h}, 50^{\circ} \mathrm{C}$.

mPEG750- $\mathrm{C}_{10} \mathrm{COOH}$ (1). In a $50 \mathrm{~mL}$ round-bottom flask, mPEG750-COOTFP (1.09 g, 1.21 $\mathrm{mmol}$ ) and 11-aminoundecanoic acid (276 mg, $1.33 \mathrm{mmol}, 1.1 \mathrm{eq})$ were dissolved in dioxane / $\mathrm{H}_{2} \mathrm{O}$ 1:1 (8 mL). N,N-diisopropylethylamine $(700 \mu \mathrm{L}, 4.0 \mathrm{mmol}, 3 \mathrm{eq}$ to the free acid) was added and the solution was stirred at r.t. for $1 \mathrm{~h}$, during which it became almost clear. Concentrated $\mathrm{HCl}$ and $0.1 \mathrm{M} \mathrm{HCl}(50 \mathrm{~mL})$ were added to $\mathrm{pH} \sim 1$ and the aqueous phase was extracted with dichloromethane $(3 \times 25 \mathrm{~mL})$. The combined organic layers were dried using $\mathrm{MgSO}_{4}$, filtrated and the solvent was removed in vacuo. The compound was purified using column chromatography (flash $\mathrm{SiO}_{2}$ ) using an elution gradient of $2 \% \mathrm{MeOH}$ in chloroform to $10 \% \mathrm{MeOH}$ in chloroform to $(15 \% \mathrm{MeOH}+1 \%$ formic acid) in chloroform. This yielded pure $\mathbf{1}(750 \mathrm{mg}, 0.80 \mathrm{mmol}, 66 \%)$ as a white solid. ${ }^{1} \mathrm{H}$ NMR: $\delta=7.00(\mathrm{~m}, 1 \mathrm{H}, \mathrm{NH}), 3.98\left(\mathrm{~s}, 2 \mathrm{H}, \mathrm{OCH}_{2} \mathrm{CO}\right), 3.70-3.55(\mathrm{~m}, 60 \mathrm{H}$, $\mathrm{OCH}_{2}$ ), 3.37 (s, $\left.3 \mathrm{H}, \mathrm{CH}_{3}\right), 3.27\left(\mathrm{q}, 2 \mathrm{H}, \mathrm{NHCH}_{2}\right), 2.31\left(\mathrm{t}, 2 \mathrm{H}, \mathrm{CH}_{2} \mathrm{COOH}\right), 1.62(\mathrm{~m}, 2 \mathrm{H}$, 
$\left.\mathrm{CH}_{2} \mathrm{CH}_{2} \mathrm{COOH}\right), 1.51\left(\mathrm{~m}, 2 \mathrm{H}, \mathrm{NHCH}_{2} \mathrm{CH}_{2}\right), 1.28\left(\mathrm{~m}, 12 \mathrm{H}\right.$, remaining $\left.\mathrm{CH}_{2}\right) .{ }^{13} \mathrm{C}-\mathrm{NMR}: \delta=$ 177.1, 170.0, 71.9, 70.9, 70.6, 70.52, 70.46, 70.3, 70.2, 59.0, 38.9, 33.9, 29.5, 29.3, 29.2, 29.1, 29.0, 28.9, 26.8, 24.7. ESI-MS: $m / z$ Calc. 934.17; Obs. $[\mathrm{M}+2 \mathrm{H}]^{2+} 468.00$ (mass envelopes with $\Delta m / z=$ 44/z). FT-IR: $v\left(\mathrm{~cm}^{-1}\right)=3331,3258,2916,2877,2739,1703,1660,1648,1549,1465,1436$, $1412,1342,1304,1279,1260,1241,1219,1200,1146,1106,1062,958,899,843,800,759,741$, $728,718,681,614$.

GSPPI- $C_{11}-m P E G 750$ (2). In a $100 \mathrm{~mL}$ round-bottom flask, G5PPI $\left(\mathrm{NH}_{2}\right)_{64}(115 \mathrm{mg}, 16 \mu \mathrm{mol}), \mathbf{1}$ (1.2 g, $1.25 \mathrm{mmol}, 1.25$ eq per $\left.\mathrm{NH}_{2}\right)$ and $N, N$-diisopropylethylamine $(680 \mu \mathrm{L}, 3.9 \mathrm{mmol}, 3$ eq to 1) were dissolved in dichloromethane $(15 \mathrm{~mL})$. After the addition of PyBOP $(683 \mathrm{mg}, 1.29 \mathrm{mmol}$, 1.26 eq per $\mathrm{NH}_{2}$ ) and dichloromethane $(2 \mathrm{~mL})$ the solution was stirred at room temperature for 1 h. Chloroform $(150 \mathrm{~mL})$ was added and the solution was washed with $0.1 \mathrm{M} \mathrm{NaOH}(40 \mathrm{~mL})$ and gently with $\mathrm{H}_{2} \mathrm{O}(40 \mathrm{~mL})$. The organic layer was dried using $\mathrm{Na}_{2} \mathrm{SO}_{4}$, filtrated and the solvent was removed in vacuo. Preparative size-exclusion chromatography (BioBeads SX-1, 10\% $\mathrm{MeOH}$ in $\left.\mathrm{CHCl}_{3}\right)$ was performed and the compound was redissolved in $\mathrm{CHCl}_{3}(100 \mathrm{~mL})$. The organic layer was washed with $0.1 \mathrm{M} \mathrm{NaOH}(30 \mathrm{~mL})$, dried using $\mathrm{Na}_{2} \mathrm{SO}_{4}$, filtrated and the solvent was removed in vacuo. Preparative size-exclusion chromatography (BioBeads SX-1, 10\% $\mathrm{MeOH}$ in $\left.\mathrm{CHCl}_{3}\right)$ was again performed. Finally, precipitation $\left(2 \frac{1 / 2}{\mathrm{~mL}}\right.$ dichloromethane $\rightarrow 180 \mathrm{~mL}$ diethylether, subsequently stored at r.t. for $2 \mathrm{~h}$ ) was able to remove excess $\mathbf{1}$ and yielded pure $\mathbf{2}$ (880 mg, $13.4 \mu \mathrm{mol}, 84 \%$ ) as a slightly orange solid. ${ }^{1} \mathrm{H}-\mathrm{NMR}: \delta=7.65$ (br, NHCO), 6.98 (br, $\mathrm{NHCO}$ ), 3.97 (s, $\mathrm{OCH}_{2} \mathrm{CO}$ ), 3.85-3.52 (m, $\left.\mathrm{CH}_{2} \mathrm{O}\right), 3.38$ (s, $\left.\mathrm{CH}_{3}\right), 3.24$ (q, $\left.\mathrm{CH}_{2} \mathrm{NHCO}\right), 2.36$ (br, $\mathrm{CH}_{2} \mathrm{~N}$ ), 2.18 (br, $\left.\mathrm{NHCOCH}_{2} \mathrm{CH}_{2}\right), 1.68-1.46$ (br, $\mathrm{NCH}_{2} \mathrm{CH}_{2}, \mathrm{NHCOCH}_{2} \mathrm{CH}_{2}$, $\mathrm{NHCH}_{2} \mathrm{CH}_{2}$ ), 1.27 (br, remaining $\mathrm{CH}_{2}$ ). ${ }^{13} \mathrm{C}-\mathrm{NMR}: \delta=173.9,169.6,71.9,70.9,70.6,70.5,70.3$, 59.0, 52.2 (br), 51.1 (br), 38.9, 37.5 (br), 36.5 (br), 29.7, 29.6, 29.4, 27.0, 26.0, 24.7 (br). FT-IR: $v$ $\left(\mathrm{cm}^{-1}\right)=3285,2915,2882,2742,1648,1639,1548,1466,1359,1344,1280,1242,1198,1146$, $1104,1061,963,842,721$.

G5(TEMPO) $)_{x}(x=1,5,10,20)$. General procedure for the quaternization (Tack et al., 2006), illustrated for $\mathrm{x}=1$ : In a GC-MS vial, $2(36 \mathrm{mg}, 0.55 \mu \mathrm{mol})$ and 4-((2-iodoacetyl)amino)-2,2,6,6tetramethyl-1-piperidinyloxy $(184 \mu \mathrm{g}, 0.54 \mu \mathrm{mol}, 1 \mathrm{eq})$ were dissolved in methanol $(400 \mu \mathrm{L})$ and the mixture was stirred at $50{ }^{\circ} \mathrm{C}$ for $66 \mathrm{~h}$. The solvent was removed in vacuo and BioBeads SX-1 $\left(10 \% \mathrm{MeOH}\right.$ in $\left.\mathrm{CHCl}_{3}\right)$ yielded pure G5(TEMPO) $)_{1}(36 \mathrm{mg}, 0.55 \mu \mathrm{mol}, 100 \%)$ as a slightly orange solid. The ${ }^{1} \mathrm{H}-\mathrm{NMR}$ spectra of G5(TEMPO) $)_{\mathbf{x}}$ are similar to that of $\mathbf{2}$, but becomes increasingly broad and undefined with increasing $\mathrm{x}$. 


\section{S5.1.2 Synthesis of ATTO-labeled dendrimers}

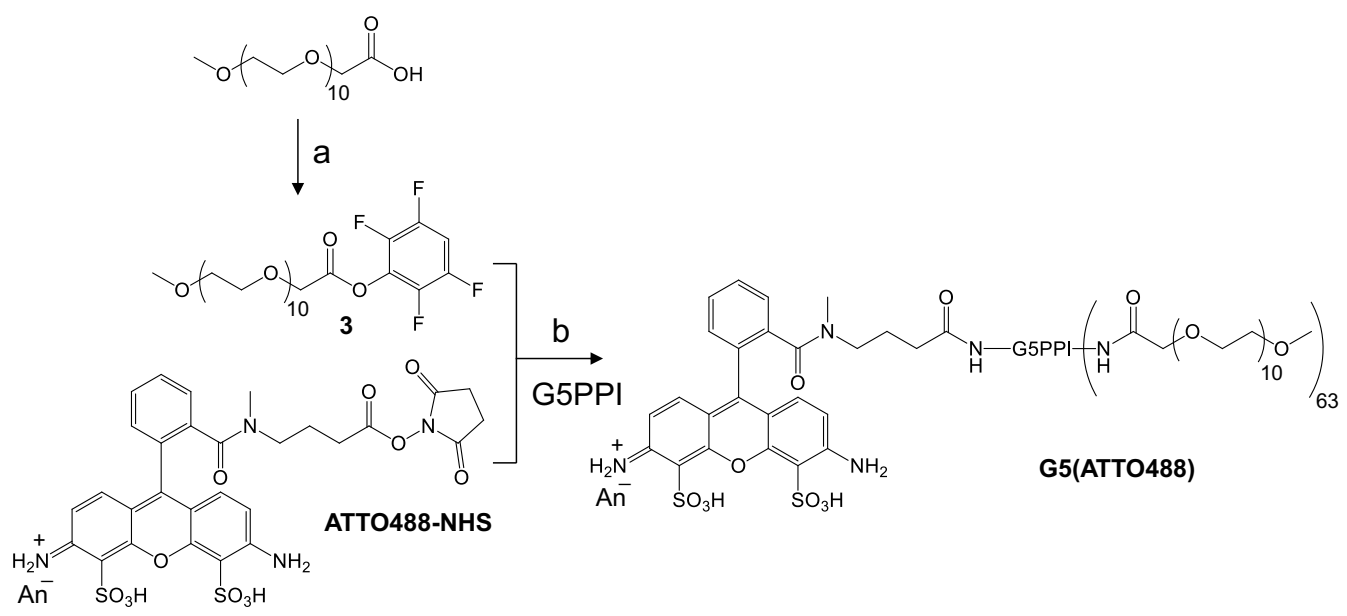

Scheme S5.2: Synthesis of generation 5 dendritic nanoparticles with a monodisperse mPEG coating and (on average) one ATTO488 dye per nanoparticle (a) 2,3,5,6-tetrafluorophenol, pyridinium ptoluenesulfonate, 1-ethyl-3-(3-dimethylaminopropyl)carbodiimide hydrochloride, $\mathrm{CH}_{2} \mathrm{Cl}_{2}, 1 \mathrm{~h}$, r.t., $100 \%$; ii. G5PPI $\left(\mathrm{NH}_{2}\right)_{64}, \mathrm{~N}, \mathrm{~N}$-diisopropylethylamine, DMF, 1 h, r.t., $96 \%$.

mPEG10-COOTFP (3). In a $50 \mathrm{~mL}$ round-bottom flask, 3,6,9,12,15,18,21,24,27,30,33undecaoxatetratriacontanoic acid (140 mg, $0.26 \mathrm{mmol}$ ), 2,3,5,6-tetrafluorophenol (54 mg, 0.32 mmol, $1.2 \mathrm{eq})$ and pyridinium $p$-toluenesulfonate $(7 \mathrm{mg}, 27 \mu \mathrm{mol}, 0.1 \mathrm{eq})$ were dissolved in $\mathrm{CH}_{2} \mathrm{Cl}_{2}(2 \mathrm{~mL})$. 1-Ethyl-3-(3-dimethylaminopropyl)carbodiimide hydrochloride $(62 \mathrm{mg}, 0.32$ mmol, $1.2 \mathrm{eq})$ was added and the solution was stirred at r.t. for $1 \mathrm{~h} . \mathrm{CHCl}_{3}(60 \mathrm{~mL})$ was added and the solution was washed with $\mathrm{NaHCO}_{3}$ (sat) and brine (both $20 \mathrm{~mL}$ ). The organic layer was dried with $\mathrm{MgSO}_{4}$, filtrated and the solvent was removed in vacuo. This yielded pure 3 (200 mg, >100\%, contains solvent) as a colorless oil, which was used immediately without further purification. ${ }^{1} \mathrm{HNMR}: \delta=7.03(\mathrm{~m}, 1 \mathrm{H}, \mathrm{ArH}), 4.56\left(\mathrm{~s}, 2 \mathrm{H}, \mathrm{CH}_{2} \mathrm{COO}\right), 3.85-3.53\left(\mathrm{~m}, 40 \mathrm{H}, \mathrm{OCH}_{2}\right), 3.38(\mathrm{~s}, 3 \mathrm{H}$, $\left.\mathrm{OCH}_{3}\right) .{ }^{19} \mathrm{~F}-\mathrm{NMR}: \delta=-138.6,-152.7$.

G5(ATTO488). In a $5 \mathrm{~mL}$ tube flask, G5PPI $\left(\mathrm{NH}_{2}\right)_{64}(25 \mathrm{mg}, 3.5 \mu \mathrm{mol})$ and $\mathrm{N}, \mathrm{N}$ diisopropylethylamine ( $140 \mu \mathrm{L}, 0.78 \mathrm{mmol}, 3$ eq to the total active ester content) were dissolved in DMF ( $1 \mathrm{~mL}$ ). Subsequently, 3 (90 mg, $0.13 \mathrm{mmol}, 38 \mathrm{eq}$ ), ATTO488 (4.0 mg, $4.1 \mu \mathrm{mol}, 1.2$ eq) and more 3 (90 mg, $0.13 \mathrm{mmol}, 38 \mathrm{eq}$ ) were consecutively added. After each addition of active ester, the mixture was stirred at r.t. for $20 \mathrm{~min}$. DMF was then removed in vacuo (oil pump, $40^{\circ} \mathrm{C}$ ) and chloroform $(60 \mathrm{~mL})$ was added. The solution was washed with $0.1 \mathrm{M} \mathrm{NaOH}(20 \mathrm{~mL})$, dried using $\mathrm{Na}_{2} \mathrm{SO}_{4}$, filtrated and the solvent was removed in vacuo. Preparative size-exclusion chromatography (BioBeads $\mathrm{SX}-1,10 \% \mathrm{MeOH}$ in $\mathrm{CHCl}_{3}$ ) yielded pure G5(ATTO488) (135 mg, 
$3.4 \mu \mathrm{mol}, 96 \%$ ) as a red oil. ${ }^{1} \mathrm{H}-\mathrm{NMR}: \delta=7.28$ (br, $\mathrm{NHCO}$ ), 3.96 (s, $\left.\mathrm{OCH}_{2} \mathrm{CO}\right), 3.72-3.53$ (m, $\mathrm{CH}_{2} \mathrm{O}$ ), 3.38 (s, $\mathrm{OCH}_{3}$ ), 3.28 (br, $\mathrm{CH}_{2} \mathrm{NHCO}$ ), $2.40\left(\mathrm{br}, \mathrm{CH}_{2} \mathrm{~N}\right), 1.69-1.47$ (br, $\mathrm{NCH}_{2} \mathrm{CH}_{2}$ ), the dye protons are not visible. ${ }^{13} \mathrm{C}-\mathrm{NMR}: \delta=169.6,71.9,71.8(\mathrm{br}), 70.7,70.54,70.50,70.47,70.46$, 70.3, 69.4 (br), 59.0, 52.4 (br), 51.9 (br), 51.4, 37.3, 27.1, 24.1 (br). FT-IR: $v\left(\mathrm{~cm}^{-1}\right)=3344,2866$, $1669,1532,1454,1349,1325,1296,1249,1199,1097,1041,946,850,657$. 


\section{S5.2 Exponential fitting of G5-dendrimer ${ }^{1} \mathrm{H}$ NMR attenuation curves: goodness of fit}

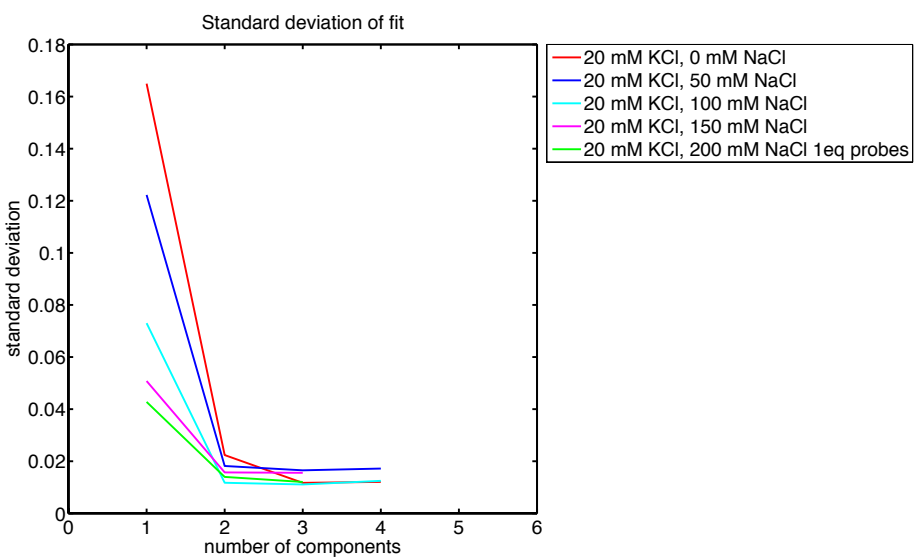

Figure S5.1: Goodness of fit of ${ }^{1} \mathrm{H}$ NMR diffusometry data of G5 particles in $2 \mathrm{wt} \% \mathrm{\kappa}$-carrageenan gels. A sum of two exponentials appears to describe the curves, except in case of $0 \mathrm{mM} \mathrm{Na}^{+}$, where an additional component can be added. 


\section{S5.3 ${ }^{1} \mathrm{H}$ NMR attenuation curves for $\mathrm{G} 1$ and $\mathrm{G} 3$ dendrimers in $2 \mathrm{wt} \%$ k-carrageenan}

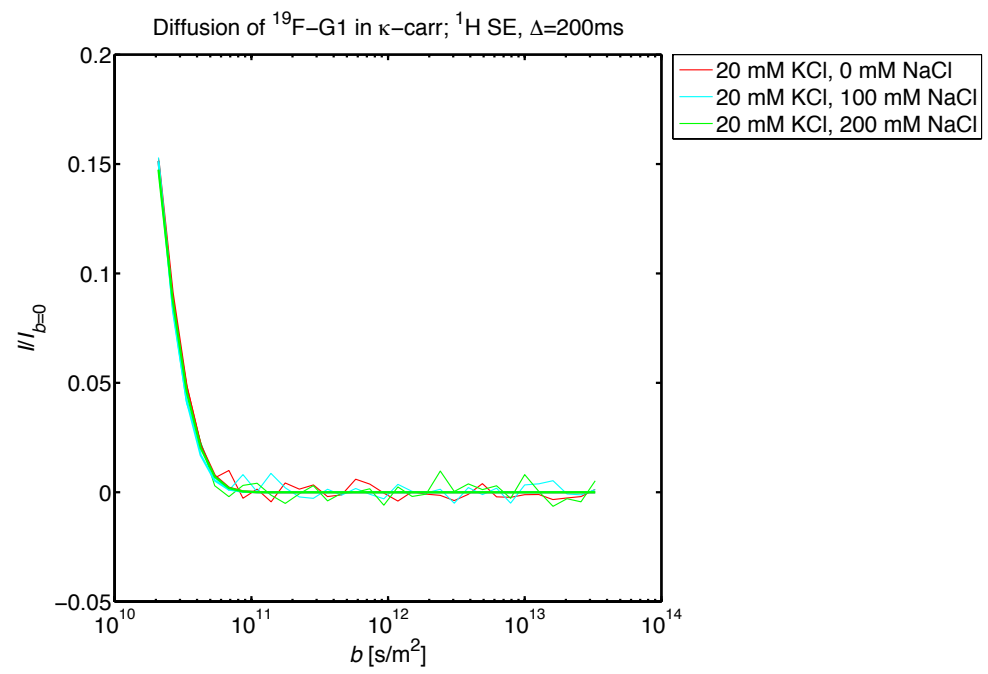

Figure S5.2: No significant slow component for G1 dendrimers in $2 \mathrm{wt} \% \mathrm{\kappa}$-carrageenan gels at different salt concentrations.

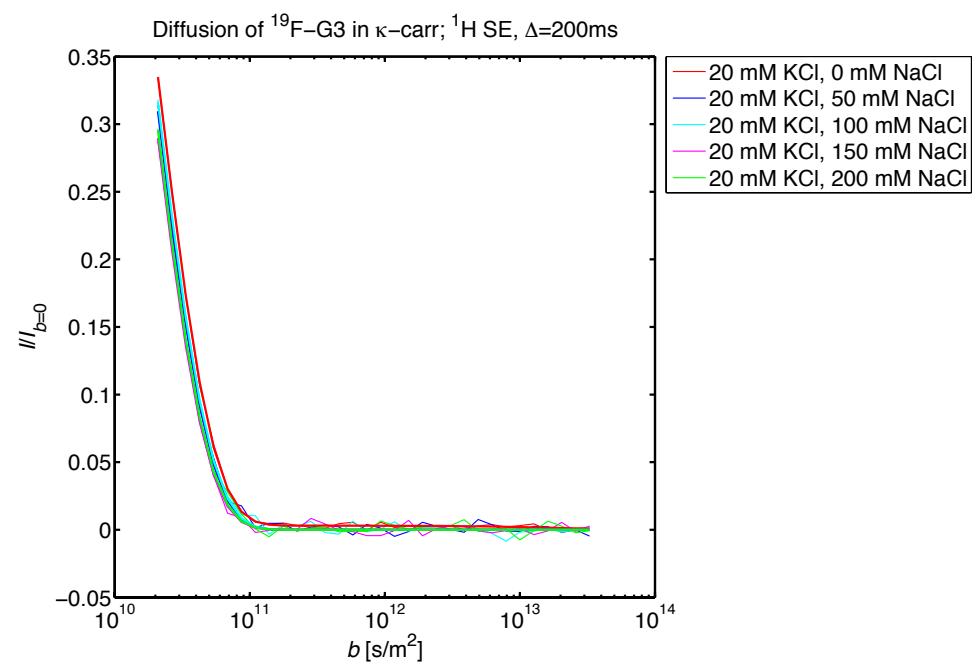

Figure S5.3: No significant slow component for G3 dendrimers in $2 \mathrm{wt} \% \mathrm{k}$-carrageenan gels at different salt concentrations (a minor offset is visible only at $0 \mathrm{mM} \mathrm{Na}^{+}$). 


\section{References}

Armstrong, B. D., \& Han, S. (2007). A new model for Overhauser enhanced nuclear magnetic resonance using nitroxide radicals. Journal of Chemical Physics, 127(10). http://doi.org/10.1063/1.2770465

Armstrong, B. D., \& Han, S. (2009). Overhauser Dynamic Nuclear Polarization To Study Local Water Dynamics. Journal of the American Chemical Society, 131(13), 4641-4647. http://doi.org/10.1021/ja809259q

Bourouina, N., de Kort, D. W., Hoeben, F. J. M., Janssen, H. M., Van As, H., Hohlbein, J., et al. (2015). Complex Coacervate Core Micelles with Spectroscopic Labels for Diffusometric Probing of Biopolymer Networks. $\quad$ Langmuir, 31(46), 12635-12643. http://doi.org/10.1021/acs.langmuir.5b03496

de Kort, D. W., Hoeben, F. J. M., Janssen, H. M., Bourouina, N., Kleijn, J. M., van Duynhoven, J. P. M., \& Van As, H. (2015a). 19F Labelled Polyion Micelles as Diffusional Nanoprobes. In F. Capozzi, L. Laghi, \& P. S. Belton (Eds.), Magnetic Resonance in Food Science. Cambridge, UK: The Royal Society of Chemistry. http://doi.org/10.1039/9781782622741-00109

de Kort, D. W., Rombouts, W. H., Hoeben, F. J. M., Janssen, H. M., Van As, H., \& van Duynhoven, J. P. M. (2015b). Scaling Behavior of Dendritic Nanoparticle Mobility in Semidilute Polymer Solutions. Macromolecules, 48(20), 7585-7591. http://doi.org/10.1021/acs.macromol.5b01530

de Kort, D. W., van Duynhoven, J. P. M., Hoeben, F. J. M., Janssen, H. M., \& Van As, H. (2014). NMR nanoparticle diffusometry in hydrogels: enhancing sensitivity and selectivity. Analytical Chemistry, 86(18), 9229-9235. http://doi.org/10.1021/ac502211q

de Kort, D. W., van Duynhoven, J. P. M., Van As, H., \& Mariette, F. (2015c). Nanoparticle diffusometry for quantitative assessment of submicron structure in food biopolymer networks. Trends in Food Science \& Technology, 42(1), 13-26. http://doi.org/10.1016/j.tifs.2014.11.003

Digman, M. A., Sengupta, P., Wiseman, P. W., Brown, C. M., Horwitz, A. R., \& Gratton, E. (2005). Fluctuation correlation spectroscopy with a laser-scanning microscope: exploiting the hidden time structure. Biophysical Journal, 88(5), L33-6. http://doi.org/10.1529/biophysj.105.061788

Franck, J. M., Pavlova, A., Scott, J. A., \& Han, S. (2013). Quantitative cw Overhauser effect dynamic nuclear polarization for the analysis of local water dynamics. Progress in Nuclear Magnetic Resonance Spectroscopy, 74(C), 33-56. http://doi.org/10.1016/j.pnmrs.2013.06.001

Hattori, K., Okada, T., Kondoh, O., \& Tsukaguchi, T. (2009). 1-(2H)-isoquinolone derivative. US Patent Office. Retrieved from http://www.google.com/patents/US20090030195

Hermansson, A. M. (1989). Rheological and Microstructural Evidence for Transient States During Gelation of Kappa-Carrageenan in the Presence of Potassium. Carbohydrate Polymers, 10(3), 163-181. http://doi.org/10.1016/0144-8617(89)90009-X

Hermansson, A. M., Eriksson, E., \& Jordansson, E. (1991). Effects of potassium, sodium and calcium on the microstructure and rheological behaviour of kappa-carrageenan gels. Carbohydrate Polymers, 16(3), 297-320. http://doi.org/10.1016/0144-8617(91)90115-S

Lorén, N., Nydén, M., \& Hermansson, A.-M. (2009a). Determination of local diffusion properties in heterogeneous biomaterials. Advances in Colloid and Interface Science, 150(1), 5-15. http://doi.org/10.1016/j.cis.2009.05.004

Lorén, N., Shtykova, L., Kidman, S., Jarvoll, P., Nydén, M., \& Hermansson, A.-M. (2009b). Dendrimer diffusion in kappa-carrageenan gel structures. Biomacromolecules, 10(2), 275-284. http://doi.org/10.1021/bm801013x

Masaro, L., \& Zhu, X. (1999). Physical models of diffusion for polymer solutions, gels and solids. Progress in Polymer Science, 24(5), 731-775. http://doi.org/10.1016/S0079-6700(99)00016-7

Ortony, J. H., Cheng, C.-Y., Franck, J. M., Kausik, R., Pavlova, A., Hunt, J., \& Han, S. (2011). Probing the hydration water diffusion of macromolecular surfaces and interfaces. New Journal of Physics, 13(1). http://doi.org/10.1088/1367-2630/13/1/015006 
Stejskal, E. O., \& Tanner, J. E. (1965). Spin diffusion measurements: Spin echoes in the presence of a timedependent field gradient. The Journal of Chemical Physics, 42, 288-292. http://doi.org/10.1063/1.1695690

Tack, F., Bakker, A., Maes, S., Dekeyser, N., Bruining, M., Elissen-Roman, C., et al. (2006). Modified poly(propylene imine) dendrimers as effective transfection agents for catalytic DNA enzymes (DNAzymes). Journal of Drug Targeting, 14(2), 69-86. http://doi.org/10.1080/10611860600635665

Viebke, C., Piculell, L., \& Nilsson, S. (1994). On the Mechanism of Gelation of Helix-Forming Biopolymers. Macromolecules, 27(15), 4160-4166. http://doi.org/10.1021/ma00093a017 



\title{
Scaling behavior of dendritic nanoparticle mobility in semidilute polymer solutions
}

\begin{abstract}
In our studies on particle mobility in polymer solutions, we have investigated and determined selfdiffusion coefficients of nanoparticles in semidilute solutions of poly(ethylene glycol) (PEG, $M_{\mathrm{w}}=6,20,35$ and $100 \mathrm{kDa}$ ). Specially designed PEGylated dendrimers with well-defined sizes $\left(d_{\mathrm{h}}=3.4-11.0 \mathrm{~nm}\right)$, and with internal ${ }^{19} \mathrm{~F}$ moieties allow for background-free ${ }^{19} \mathrm{~F}$ NMR diffusion measurements. This way, we were able to assess the self-diffusion coefficients as a function of particle diameter, and length scales (correlation length, tube diameter, polymer radius of gyration) with high resolution. Scaling arguments allowed us to visualize a clear crossover between particles probing a lower apparent viscosity to near-macroviscosity when the nanoparticle size is comparable to the PEG polymer coil size. The same arguments are shown to correctly predict particle diffusion coefficients as a function of polymer concentration when the particles are smaller than the polymer coils.
\end{abstract}

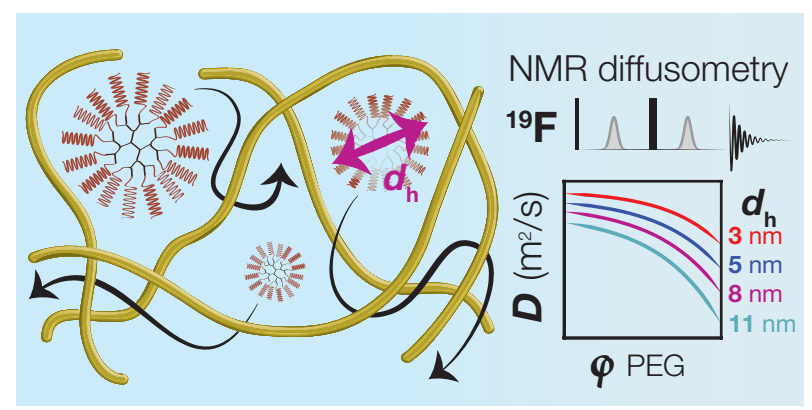

Daan W. de Kort, Wolf H. Rombouts, Freek J.M. Hoeben, Henk M. Janssen, Henk Van As, and John P.M. van Duynhoven, Macromolecules 2015, 48 (20), pp 7585-7591. DOI: 10.1021/acs.macromol.5b01530 


\subsection{Introduction}

Mobility of spherical particles in polymer networks is an active area of both fundamental and applied research. Detailed knowledge on the particle mobility in such networks can build more understanding of both microscopic (heterogeneity) and macroscopic (rheological) material behavior and properties (Mason \& Weitz, 1995; Valentine et al., 2001) Other research drivers are the recent interest in anomalous transport properties of macromolecules in crowded biological cells (Höfling \& Franosch, 2013) and porous media (Novikov, Fieremans, Jensen, \& Helpern, 2011), or the mobility of particles in polymer nanocomposites (Kumar, Jouault, Benicewicz, \& Neely, 2013). In solutions of polymers, the macroscopic viscosity $\eta_{\mathrm{m}}$ can be several orders of magnitude higher than that of the constituent solvent $\left(\eta_{0}\right)$ even at low polymer concentrations. In the limit of small particle diameters $d$, embedded particles will experience the solvent viscosity. Particles that are large compared to colloidal length scales, on the other hand, will experience macroscopic viscosity. So in these limiting cases, the Stokes-Einstein (SE) relation $D=k_{b} T /(3 \pi \eta d)$, where $k_{b}$ is the Boltzmann constant and $T$ the absolute temperature, predicts the long-term self-diffusion coefficients $D$. Of particular interest is particle diffusion in the "mesoscopic" regime, where the particle diameter is on the order of the "crossover" length scale between the solvent viscosity and macroviscosity regimes. This is typically the case for nanometersize particles. For the case of semidilute entangled polymer solutions, several physical parameters have been proposed to be the determinants of particle dynamics in the mesoscopic regime. The onset of the semidilute regime is defined as the volume fraction $\varphi^{*}$ at which polymer coils begin to overlap, and above which the solution can be described as a transient network with correlation length $\xi$. The correlation length is defined as the average distance between the two adjacent contact points between chains, and depends on polymer volume fraction $\varphi$ as $\xi(\varphi) \sim \varphi^{-v /(3 v-1)} \simeq \varphi^{-}$ ${ }^{3 / 4}$ ( $v=3 / 5$ for a swollen chain) (de Gennes, 1976; 1979). Between contact points, polymers adopt a spherical "blob" conformation; the size of the blobs is $\xi$. Although $\varphi \ll 1$, the monomer density in semidilute solutions is much more homogeneous than in dilute solution. Semidilute solutions have been compared to a porous "sponge" in which solvent (and particles) flow (Cukier, 1984). In early experimental work, Langevin and Rondelez used sedimentation experiments to investigate particle mobility in semidilute solutions and claimed that particle sedimentation coefficients $s$ scale as a function of $(d / \xi)$ (Langevin \& Rondelez, 1978). They proposed that $s \sim 1$ for $d \ll \xi$ and otherwise $s \sim \exp \left[-A(d / \xi)^{y}\right] \simeq \exp \left[-A\left(d \phi^{3 / 4}\right)^{y}\right]$ : a stretched exponential function where $A$ and $y$ are adjustable parameters. In this empirical model, particle mobility is mainly governed by topological constraints, and the correlation length (blob size) $\xi$ is put forward as the length scale at which particle diffusion is influenced by the polymers network. Cukier (Cukier, 1984) and Altenberger (Altenberger, Tirrell, \& Dahler, 1986) tried to find more arguments for this behavior: Cukier stated that $\left(D / D_{0}\right) \sim \exp [-\kappa d]$, where $D_{0}$ is the diffusion coefficient in pure solvent and $\kappa$ a constant relating to the screening length, by describing semidilute solutions 
hydrodynamically as solutions of spheres (i.e., correlation blobs) with Debye-Bueche screening (Brinkman, 1949; Debye \& Bueche, 1948). If these spheres do not move on the time scale of particle motion, $\kappa \sim \varphi^{3 / 4}$ in good solvent. Altenberger et al. found $\left(D / D_{0}\right) \sim \exp \left[-c^{n}\right]$, where $c$ is the concentration of obstacles and exponent $n$ ranges between 0.5 and 1 , through an approach in which polymer blobs were again modeled as stationary points of friction. The stretchedexponential dependence of diffusion coefficients on polymer concentration has been confirmed by many studies dating back to the 80s, but the question of "crossover" between the regimes where nanoparticles probe low apparent viscosity and macroviscosity was not thoroughly dealt with until recently (Cai, Panyukov, \& Rubinstein, 2011; 2015; Kalwarczyk et al., 2015).

Alternative to the hydrodynamic models, a scaling model was proposed by Cai et al. (Cai et al., 2011), based on initial work by Brochard Wyart and de Gennes (Brochard Wyart \& de Gennes, 2000). Cai et al. suggested that blob size $\xi$, tube diameter $a$ and radius of gyration $R_{g}$ are the length scales determining particle mobility in semidilute polymer solutions. Terminal self-diffusion coefficients of particles with size $d<R_{g}$ follow scaling laws in three different regimes $(d<\xi$, $\xi<d<a, a<d)$. For $d<\xi$, the nanoparticles probe only solvent viscosity $\eta_{0}$ and the long-time selfdiffusion coefficient therefore is independent of $\varphi, D(\varphi) \sim 1$. In the $\xi<d<a$ regime, particle mobility is affected by the correlation blobs of size $\xi$, so that self-diffusion coefficients are proportional to $k_{b} T \xi^{2} /\left(\eta_{0} d^{3}\right)$, as the "effective viscosity" is a factor $(d / \xi)^{2}$ higher than in the $d<\xi$ case. Since $\xi(\varphi) \sim \varphi^{-0.76}$ (they use $v \approx 0.588$ (Le Guillou \& Zinn-Justin, 1977)) it follows that $D(\varphi) \sim \varphi^{-1.52}$ in good solvent. In the $d>a$ case, particles will be trapped in the entangled polymer network at short time scales, and can only move at time scales larger than the entanglement relaxation time. At the crossover point $a$, a jump in viscosity should occur, after which particles experience the macroscopic viscosity $\eta_{\mathrm{m}}$ so that the terminal self-diffusion coefficient becomes proportional to $k_{b} T /\left(\eta_{\mathrm{m}} d\right)$. For entangled polymer solutions, the macroscopic viscosity scales as $\eta_{\mathrm{m}} \sim \phi^{3 /(3 v-1)} \simeq \phi^{3.93}$ in good solvent (Colby \& Rubinstein, 1990), so $D(\varphi) \sim \varphi^{-3.93}$. In their more recent work they showed that the jump at $d=a$ is smoothened by particle hopping and particles should not feel full macroviscosity until they are several times larger than $a$. (Cai et al., 2015)

Kohli and Mukhopadhyay (Kohli \& Mukhopadhyay, 2012) have reported self-diffusion coefficients of colloidal gold particles $(<20 \mathrm{~nm})$ in semidilute solutions of poly(ethylene glycol) (PEG) in water, and found their data to be in agreement with this scaling model. More recent work showed that the transition between the $\xi<d<a$ and $a<d$ regimes is indeed not sharp, and that the particle diameter should exceed at least a few times the tube diameter to experience the macroscopic viscosity (Grabowski \& Mukhopadhyay, 2014).

A crossover to macroviscosity $\eta_{\mathrm{m}}$ is not only expected for particles for which $d<R_{g}$ at $d=a$, but also for $\xi<d<a$ particles at $d=R_{g}$. When such intermediate-sized particles are smaller than the polymers, their diffusion coefficients are independent of polymer size. If they are larger than the polymers, their diffusion coefficients scale with the degree of polymerization as $D(N) \sim k_{b} T /\left(\eta_{0} d N\right)$, where $N$ is the number of monomers in the polymer chains. A similar 
polymer weight-dependency of the crossover point has been described by Hołyst and coworkers (Hołyst et al., 2009; Kalwarczyk et al., 2015; Ziębacz, Wieczorek, Kalwarczyk, Fiałkowski, \& Hołyst, 2011), and Kohli and Mukhopadhyay (Kohli \& Mukhopadhyay, 2012), who found a sharp crossover to macroviscosity when the ratio of particle diameter to polymer radius of gyration $d / R_{g} \sim 1$. They observed that terminal diffusion coefficients fall onto a single master curve $\left(D / D_{0}\right) \sim \exp \left[-a(R / \xi)^{b}\right]$, where $R=d / 2$ when $d<R_{g}$ and $R=R_{\mathrm{g}}$ when $d>R_{g}$. Fitting parameters $a$ and $b$ were of similar magnitude for polymers of different weights. This is in essence the early model by Langevin and Rondelez (Langevin \& Rondelez, 1978), with the addition of the crossover condition $d / R_{g} \sim 1$. However, Holyst et al. did not explicitly take into account the (weak) polymer concentration dependency of $R_{g}$.

In the present study, our goal is to determine terminal self-diffusion coefficients of nanoparticles in semidilute polymer solutions where the sizes of the nanoparticles are of the same magnitude as correlation length $\xi$, tube diameter $a$ and polymer size $R_{g}$. We systematically assess how (terminal) diffusion coefficients of nanoparticles scale with (1) polymer concentration, (2) polymer molecular weight and (3) nanoparticle diameter and will focus in particular on the question which conditions lead to crossover of nanoparticle diffusion towards the macroviscosity regime in semidilute solutions of PEG $\left(M_{\mathrm{w}}=6,20,35\right.$ and $\left.100 \mathrm{kDa}\right)$ in water. For this purpose, we use specially designed inert nanoparticles that allow for very accurate ${ }^{19} \mathrm{~F}$ NMR diffusion measurements.

Diffusometric methodology. Besides the more conventional methods FCS and FRAP, NMR diffusometry can be used to track Brownian motion of nanoparticles with diameters below $\sim 50 \mathrm{~nm}$. FCS relies on measurement of a temporal autocorrelation function, which is fitted with a diffusion model over several orders of magnitude in time $\left(10^{-6}-10^{3} \mathrm{~ms}\right)$. However, FCS should be used carefully when non-Fickian diffusion is expected (i.e. the diffusion coefficient varies with time), as is often the case for particles diffusing in complex media (Mason \& Weitz, 1995) and particularly when particle diffusion is expected to be influenced by the entanglement mesh. NonFickian diffusion is then reflected in the correlation curve (Ochab-Marcinek \& Holyst, 2011; Rathgeber, Beauvisage, Chevreau, Willenbacher, \& Oelschlaeger, 2009) and simple models that extract diffusion coefficients from the correlation curve presuming Fickian diffusion would then be compromised. FRAP (time scales $10^{-2}-10^{3} \mathrm{~ms}$ ) is less precise and typically used in situations where diffusion coefficients vary spatially throughout the sample (Meyvis, De Smedt, Van Oostveldt, \& Demeester, 1999). Hence, we opted for NMR diffusometry, because it allows for unequivocal determination of self-diffusion coefficients at well-defined observation times, i.e., it probes the van Hove self-correlation function (time scales of $1-10^{3} \mathrm{~ms}$ ) (Callaghan, 2011). At the same time, this method poses specific demands on the design of the nanoparticles in order for them to be visible against the background of the polymer network. For this reason, we used ${ }^{19} \mathrm{~F}$ labeled nanoparticles to allow for background-free ${ }^{19} \mathrm{~F}$ NMR diffusion measurements. 


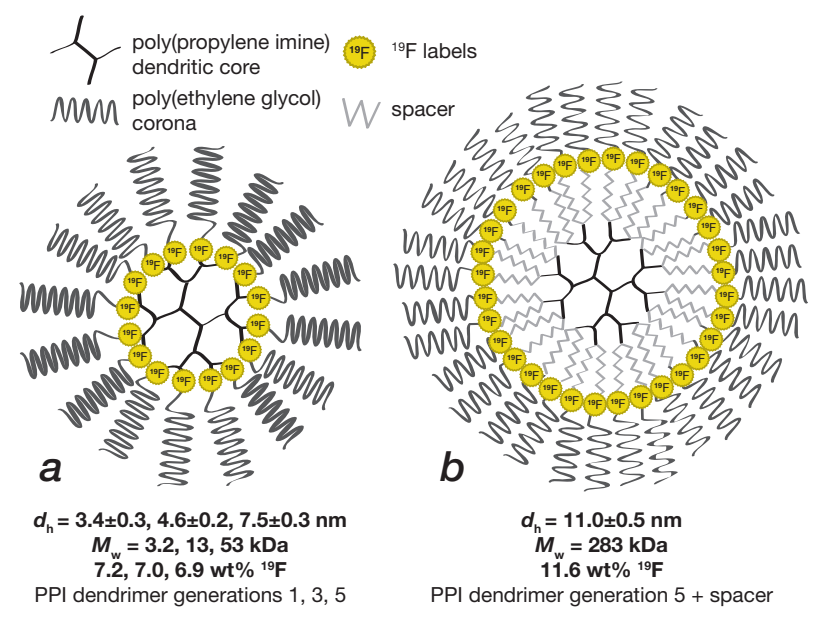

Figure 6.1: Comparison of the architecture of $(\boldsymbol{a})$ the $d_{\mathrm{h}}=3.4 \pm 0.3,4.6 \pm 0.2$ and $7.5 \pm 0.3 \mathrm{~nm}$ dendritic particles (de Kort, van Duynhoven, Hoeben, Janssen, \& Van As, 2014) and (b) the novel particle $\left(d_{\mathrm{h}}=11.0 \pm 0.5 \mathrm{~nm}\right)$. For this particle, an additional branched spacer is used to increase the size and functionality of the core, effectively creating a dendritic core of the $6^{\text {th }}$ generation. These cartoons schematically depict nanoparticle designs, but do not necessarily represent the true dynamical conformation of the particles in solution.

Selection and design of nanoparticles. To allow for a comparison of the diffusion behavior of nanoparticles with different diameters, we require them to be spherical, monodisperse in size, to have a comparable inert surface chemistry and sufficient ${ }^{19} \mathrm{~F}$-labeling to allow for ${ }^{19} \mathrm{~F}$ NMR diffusion measurements when present at low levels in polymer solutions. In previous work (de Kort et al., 2014) we have briefly described three tailor-made nanoparticles based on poly(propylene imine) (PPI) dendrimers, which vary in hydrodynamic diameter $\left(d_{\mathrm{h}}=3.4 \pm 0.3\right.$, $4.6 \pm 0.2,7.5 \pm 0.3 \mathrm{~nm}$ ), are essentially monodisperse in size, and have inert surface chemistries. In order to capture the wider range of length scales $\left(\xi, a, R_{g}\right)$, it was necessary to introduce an additional nanoparticle with a hydrodynamic diameter larger than $10 \mathrm{~nm}$ that obeyed the same design rules as the previously mentioned smaller nanoparticles. As is shown in Figure 6.1, the dendritic cores are functionalized with PEG chains to warrant water solubility of the particles, as the solubility of PPI-dendrimers is determined to a large extent by the nature of their end groups, and for avoiding specific interactions with the PEG polymers in solution. The PEG chains do not necessarily point outwards into the solution (Topp et al., 1999), but do most likely form a shell around the dendritic core (Hedden \& Bauer, 2003). The ${ }^{19} \mathrm{~F}$ moieties are placed at the interface of the dendritic core and PEG shell, and are therefore expected to be located within the shell. With this set of ${ }^{19} \mathrm{~F}$-labeled nanoparticles we expect to probe length scales in PEG polymer solutions in unsurpassed detail and address current ambiguities in hydrodynamic and scaling models. 


\subsection{Materials and Methods}

Synthesis and characterization of ${ }^{19}$ F-labeled nanoparticles. Full details on the synthesis and characterization of the generation 1, 3, 5 and "6" PPI-dendrimer nanoparticles have been included in Supporting Information section S6.1.

Preparation of PEG solutions. Polymer solutions were prepared by dissolution of $6 \mathrm{kDa}$ $\left(M_{\mathrm{w}} / M_{\mathrm{n}} 1.05\right), 20 \mathrm{kDa}\left(M_{\mathrm{w}} / M_{\mathrm{n}} 1.10\right), 35 \mathrm{kDa}\left(M_{\mathrm{w}} / M_{\mathrm{n}} 1.14\right)$ and $100 \mathrm{kDa}\left(M_{\mathrm{w}} / M_{\mathrm{n}} 1.14\right)$ PEG powder (Sigma-Aldrich) in demineralized water. The solutions were allowed to equilibrate for at least a day. For ${ }^{19}$ F NMR diffusometry measurements, nanoparticles were dosed at $0.1 \mathrm{wt} \%$.

Rheology. An Anton Paar Physica MCR 501 rheometer equipped with a Taylor-Couette geometry $(1 \mathrm{~mL})$ was used to measure flow curves of PEG solutions (without nanoparticles). Sample temperature was controlled by a thermostatic bath and was kept at $293 \mathrm{~K}$ for all experiments. Shear rate was varied within the $0.01-100 \mathrm{~s}^{-1}$ range, while ensuring that the lowest shear rates did not exceed the linear viscoelastic regime. Per decade of shear rate, 20 data points were measured. Zero-shear viscosity was estimated to be equal to the viscosity at the viscosity plateau observed for all samples at the lowest shear rates.

${ }^{19} \mathrm{~F}$ NMR nanoparticle diffusometry. ${ }^{19} \mathrm{~F}$ diffusion-ordered spectroscopy (DOSY) was performed on a Bruker Avance II spectrometer at 7.0 T magnetic field strength (resonance frequency $282 \mathrm{MHz}$ for ${ }^{19} \mathrm{~F}$ ), equipped with a Bruker diff25 gradient probe (maximum pulsed field gradient intensity $9.60 \mathrm{~T} / \mathrm{m})$. The probe was equipped with a $10-\mathrm{mm}$ RF insert tuned to the ${ }^{19} \mathrm{~F}$ resonance frequency. Sample volume was chosen as to not exceed the linear part of the magnetic field gradient. Sample temperature was kept at 293 K. DOSY experiments were performed by stepwise variation of the gradient pulse amplitude, while keeping the diffusion-observation time and gradient pulse duration constant. The attenuation of the NMR echo intensity as a function of increasing gradient amplitude is described by the Stejskal-Tanner equation $\left(I / I_{b=0}\right)=\exp (-b D)$, where $\left(I / I_{b=0}\right)$ is the echo attenuation, $D$ the self-diffusion coefficient $\left(\mathrm{m}^{2} / \mathrm{s}\right)$ and $b=(\gamma \delta g)^{2}(\Delta-$ $\delta / 3)$, where $\gamma$ is the gyromagnetic ratio of the observed nucleus $\left(25.18 \times 10^{7} \mathrm{rad} /(\mathrm{T} \mathrm{s})\right.$ for $\left.{ }^{19} \mathrm{~F}\right), g$ the magnetic field gradient amplitude $(\mathrm{T} / \mathrm{m}), \Delta$ the effective observation time $(\mathrm{s})$ and $\delta$ the effective gradient pulse duration (s), where $\delta \ll \Delta$ (narrow gradient pulse approximation).(Stejskal \& Tanner, 1965) We used stimulated echo-based DOSY experiments with unipolar, ramped gradient pulses. In all experiments, an effective observation time $\Delta$ of $100 \mathrm{~ms}$ was used, and an effective gradient pulse duration $\delta$ of $5 \mathrm{~ms}$. Gradient amplitude $g$ was varied logarithmically between $0.10-9.60 \mathrm{~T} / \mathrm{m}$ in 128 steps. The NMR signal was averaged between $16-64$ times at each gradient amplitude, with a repetition time of $1 \mathrm{~s}$. DOSY spectra were obtained through Fourier transformation of the free induction decay for each gradient step and subsequent phasing using standard procedures. The signals in each of the resulting ${ }^{19} \mathrm{~F}$ NMR spectra were integrated, and the 
resulting attenuation curve was fitted with the Stejskal-Tanner equation (non-linear least squares) using SplMod (van Resandt, Vogel, \& Provencher, 1982; Vogel, 1988). Basic bootstrapped confidence intervals of thus obtained self-diffusion coefficients were calculated by finding the median and one-standard-deviation quantiles from 1000 bootstrap samples of the attenuation curves (Davison \& Hinkley, 1997; de Kort et al., 2014).

\subsection{Results and Discussion}

\subsection{1 ${ }^{19} \mathrm{~F}$-Labeled nanoparticles with "generation 6 ” dendritic cores}

A generation-5 poly(propylene imine) dendrimer with 64 amine end groups $\left(M_{\mathrm{w}}=7.168 \mathrm{kDa}\right)$ was converted to a generation- 6 dendrimer with 192 azide end groups $\left(M_{\mathrm{w}}=72.455 \mathrm{kDa}\right)$, by attachment of a gallate based (branched) building block that bears three hexaethylene glycol spacers with azide terminal groups (cf. Figure 6.1b and Supporting Information section S6.1). Note that the molecular weight of the dendritic core remains essentially monodisperse. In the next step, and using 'click' type chemistry, an mPEG750 building block functionalized with a $-\mathrm{C}\left(\mathrm{CF}_{3}\right)_{3}$ group and a reactive alkyne group has been connected to the azide end groups of the dendritic core, resulting in formation of a larger nanoparticle with $d_{\mathrm{h}}=11.0 \pm 0.5 \mathrm{~nm}$. The ${ }^{19} \mathrm{~F}$ payload of $11.6 \mathrm{wt} \%$ compares favorably to that for the three smaller nanoparticles (ca. $7.0 \mathrm{wt} \%{ }^{19} \mathrm{~F}$ ). The difference in payload is due to the use of $-\mathrm{C}\left(\mathrm{CF}_{3}\right)_{3}$ groups in the larger nanoparticle vs. $-\mathrm{CF}_{3}$ groups in the smaller ones. The hydrodynamic diameter of $11.0 \pm 0.5 \mathrm{~nm}$ for the larger particle was calculated by the Stokes-Einstein relation from its diffusion coefficient in water at $293 \mathrm{~K}$ $\left(D=38 \pm 1 \mu \mathrm{m}^{2} / \mathrm{s}\right)$. NMR relaxation parameters at $7.0 \mathrm{~T}$ are comparable to those of the smaller nanoparticles (de Kort et al., 2014): $T_{1}\left({ }^{19} \mathrm{~F}\right)=543 \pm 24 \mathrm{~ms}, \quad T_{2}\left({ }^{19} \mathrm{~F}\right)=82 \pm 7 \mathrm{~ms}, \quad T_{1}(\mathrm{PEG}-$ $\left.{ }^{1} \mathrm{H}\right)=432 \pm 33 \mathrm{~ms}, T_{2}\left(\mathrm{PEG}-{ }^{1} \mathrm{H}\right)=294 \pm 28 \mathrm{~ms}$. This means that the current nanoparticle design allows for NMR diffusometry experiments on the $10^{1}-10^{2} \mathrm{~ms}$ timescale, for both ${ }^{1} \mathrm{H}$ and ${ }^{19} \mathrm{~F}$. A table with an overview of all relevant properties of the four different nanoparticles can be found in the Supporting Information (Table S6.1).

\subsubsection{Nanoparticle diffusion in PEG solutions}

NMR diffusometry requires that the observed nuclei in the particles have liquidlike reorientation dynamics to prevent too fast relaxation of transverse magnetization. This precludes the use of truly hard-sphere particles. The ${ }^{19} \mathrm{~F}$ labels in the particles used in this study have $T_{2} \mathrm{~s}$ in the $10^{2}-\mathrm{ms}$ range, much longer than twice the time between the first and second $90^{\circ} \mathrm{rf}$ pulses, the period during which the magnetization is affected by $T_{2}$ relaxation. Although the particles are not truly hard spheres, they can, however, be considered as spherical, and cannot change shape strongly or "unfold" into a linear conformation with changing solvent conditions or in a crowded environment. This means that we expect the variation of particle size as a function of polymer concentration to be very subtle compared to the variation of the length scales that determine their mobility. Therefore, the scaling behavior of their diffusivity in polymer solutions will not differ 
much from that of truly hard-sphere particles. Given that the time window of the NMR diffusion experiment is $1-10^{3} \mathrm{~ms}$, another prerequisite is that these time scales should be longer than all microscopic dynamic processes in the polymer network if we want to assert that truly terminal diffusion coefficients are measured. Estimations on the basis of tabulated parameters lead to the conclusion that the reptation time, i.e. the slowest microscopic process, is expected to be $\ll 10 \mathrm{~ms}$ (Supporting Information, S6.2) for PEG up to at least $100 \mathrm{kDa}$. This implies that we measure truly long-term diffusion coefficients in our experiments (100 ms diffusion observation time). Overlap and entanglement concentrations in aqueous solutions of 6, 20, $35100 \mathrm{kDa}$ PEG, correlation length $\xi$ tube diameter $a$ and polymer radii of gyration $R_{g}$ as a function of volume fraction are presented in Figure 6.2 (for details see Supporting Information, S6.3).

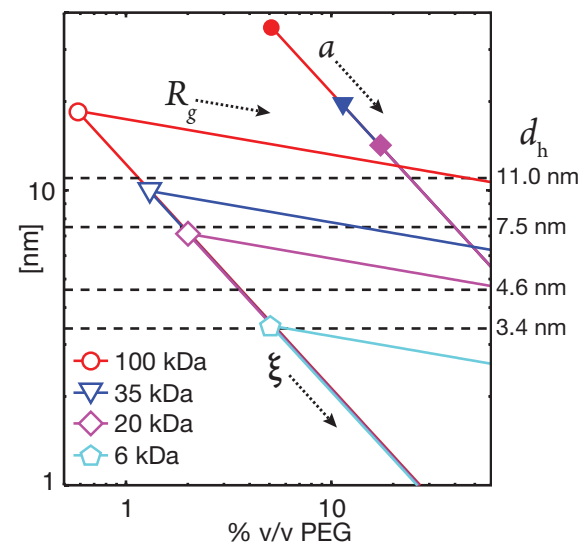

Figure 6.2: Scaling predictions of polymer network length scales as function of polymer volume fraction in aqueous solutions of 6, 20, 35 and $100 \mathrm{kDa}$ PEG. The overlap and entanglement concentrations are presented as open and filled symbols, respectively. The scaling predictions of correlation length $\xi$, tube diameter $a$ and radii of gyration $R_{g}$ with polymer volume fraction are presented as solid lines. There is no significant entanglement for 6-kDa PEG. Black (dotted) lines represent nanoparticle diameters $d_{\mathrm{h}}$.

It can be seen that the diameters of the nanoparticles used in this study are of the same order of magnitude as correlation length $\xi$, tube diameter $a$ and polymer radius of gyration $R_{g}$, i.e. they adequately probe the "mesoscopic" regime. Some representative ${ }^{19} \mathrm{~F}$ NMR echo attenuation curves measured for $d_{\mathrm{h}}=4.6 \mathrm{~nm}$ particles in $100-\mathrm{kDa}$ PEG at various volume fractions, fitted to Stejskal-Tanner attenuation curves, are shown in Figure 6.3. 


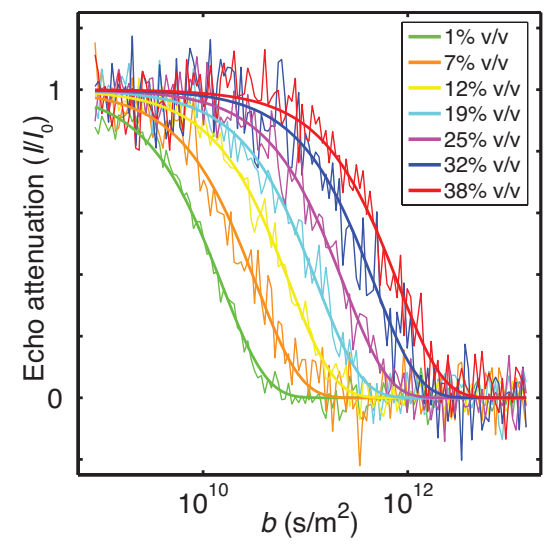

Figure 6.3 ${ }^{19} \mathrm{~F}$ DOSY echo attenuation curves for $d_{\mathrm{h}}=4.6 \mathrm{~nm}$ nanoparticles in 100-kDa PEG at various polymer volume fractions, fitted to Stejskal-Tanner attenuation curves $\left(I / I_{b=0}\right)=\exp (-b D)$.

We see that the $\mathrm{S} / \mathrm{N}$ ratio is comparable throughout the concentration range. The Stejskal-Tanner fits show that echo attenuation curves are mono-exponential, indicating a Gaussian particle displacement distribution at $\Delta=100 \mathrm{~ms}$ observation time. The error in the resulting diffusion coefficients is estimated through "bootstrap resampling" of the data points as outlined in ref (de Kort et al., 2014).

Hydrodynamic models. In Figure 6.4 we present our results and compare them to the hydrodynamic models with a stretched exponential functional form $\left(D / D_{0}\right) \sim \exp \left[-\alpha \phi^{\beta}\right]$. 

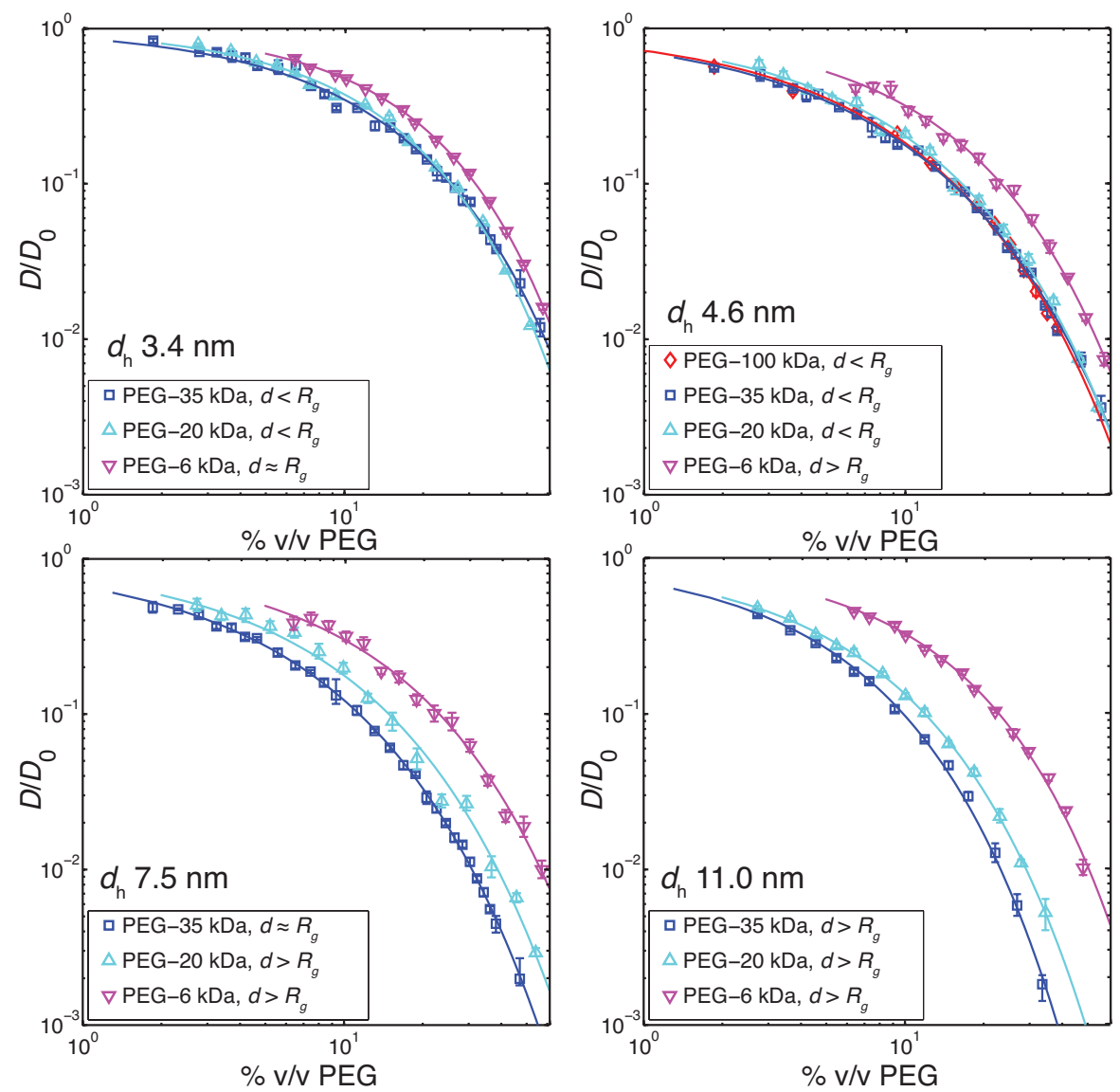

Figure 6.4: Diffusion coefficients of nanoparticles in PEG as a function of polymer volume fraction, sorted by particle diameter. Solid lines are fits to stretched exponential curves; these curves begin at the overlap concentration. It is indicated whether particle diameter $d$ is smaller or larger then the polymer coil size $R_{g}$ over the entire concentration range, or that the particle diameter is partly smaller and partly larger than the polymer coil size (cf. Figure 6.2).

The terminal diffusion coefficients of the $d_{\mathrm{h}}=3.4$ and $4.6 \mathrm{~nm}$ particles in PEG-20 kDa, $35 \mathrm{kDa}$ and $100 \mathrm{kDa}$ (only for the 4.6-nm particle) coincide within experimental error, whereas they differ significantly for PEG-6 kDa. For the $d_{\mathrm{h}}=7.5$ and $11.0 \mathrm{~nm}$ particles, none of the curves overlap. These results illustrate how particle diffusion coefficients are insensitive to the polymer coil size when $d<R_{g}$. In this limit, the Cukier and Altenberger models describe the curves closely, the average optimal value of exponent $\beta$ being $0.77 \pm 0.06$, consistent with the ideal value of $3 / 4$. This shows that there is a lower limit to the drag force that can be experienced by the nanoparticles in case they are smaller than the polymer coils. 
Now we turn towards the question whether, in case $d>R_{g}$, the nanoparticles immediately fully probe the macroscopic viscosity $\eta_{\mathrm{m}}$ such that there is a sharp "crossover condition", or whether the crossover happens more gradually. For this purpose, in Figure 6.5, we have plotted together the diffusion coefficients of particles of different sizes in the same solutions, plus the Stokes-Einstein result for the diffusion coefficients $D / D_{0}=\eta_{0} / \eta_{\mathrm{m}}$, based on solution macroviscosity $\eta_{\mathrm{m}}$ (these are presented in Supporting Information Figure S6.1).

If particles would fully probe macroviscosity for $d>R_{g}$, their diffusion coefficients would coincide with the Stokes-Einstein result. We can see, however, that is not the case for any experiment. For the lowest $d / R_{g}$ ratios, the particles diffuse up to three orders of magnitude faster than expected, while for the highest $d / R_{\mathrm{g}}$ ratios (for $d_{\mathrm{h}}=11.0 \mathrm{~nm}$ particles in PEG-6 $\mathrm{kDa}$ solutions), the difference is less than a factor of 2 (cf. Supporting Information Figure S6.2 for detailed plots of the ratio $D / D\left(\eta_{\mathrm{m}}\right)$ for all data points). On the other hand, in PEG-6 kDa, the data points of the largest three particles $\left(d / R_{g}>1.4\right)$ show no significant differences up to $\sim 40 \% \mathrm{v} / \mathrm{v}$ PEG. This lack of return to the Stokes-Einstein result with increasing particle diameter might be related to slippage of particles as predicted by Brochard-Wyart and de Gennes for non-adsorbing particles in melts. Slippage would lead to the particle diffusion coefficients being 3/2 times higher than without slippage, which would approximately account for the observed discrepancy in our experiments (Brochard Wyart \& de Gennes, 2000).

Models for the crossover condition at $d=R_{g}$. Hołyst et al. (Holyst et al., 2009; Ziębacz et al., 2011) observed that, in case particle diameter $d<R_{g}$, all points roughly fall onto a straight line in a plot of $\log \left(D / D_{0}\right)$ against $d / \xi$. Since $\xi \sim \varphi^{-3 / 4}$, this method is analogous to fitting stretched exponential curves $\left(D / D_{0}\right) \sim \exp \left[-\alpha \varphi^{\beta}\right]$, with scaling exponent $\beta=3 / 4$ and $\alpha \sim d$ in line with the predictions of Langevin, Rondelez and Cukier. This behavior, however, breaks down for $d>R_{g}$. Holyst et al. found that in this limit, data points fall onto the master curve only if we plot $\log \left(D / D_{0}\right)$ against $R_{g} / \xi$ instead of $d / \xi$. Since $R_{g} \sim \varphi^{-1 / 8}$ is a weak function of polymer volume fraction, this implies that now $\beta=5 / 8$. The model effectively implies that when $d>R_{g}$, the diffusivity of the polymer coils dictates the mobility of the particles and that the macroscopic viscosity should be proportional to $\exp \left[-\varphi^{5 / 8}\right]$. As shown in Figure 6.6, this approach works reasonably well for our data. Although the data diverge from the master curve at higher $\Upsilon / \xi$ ratios (where parameter $\Upsilon$ is either $d$ or $R_{g}$ ), the model succeeds in showing the crossover condition $d=R_{g}$. 

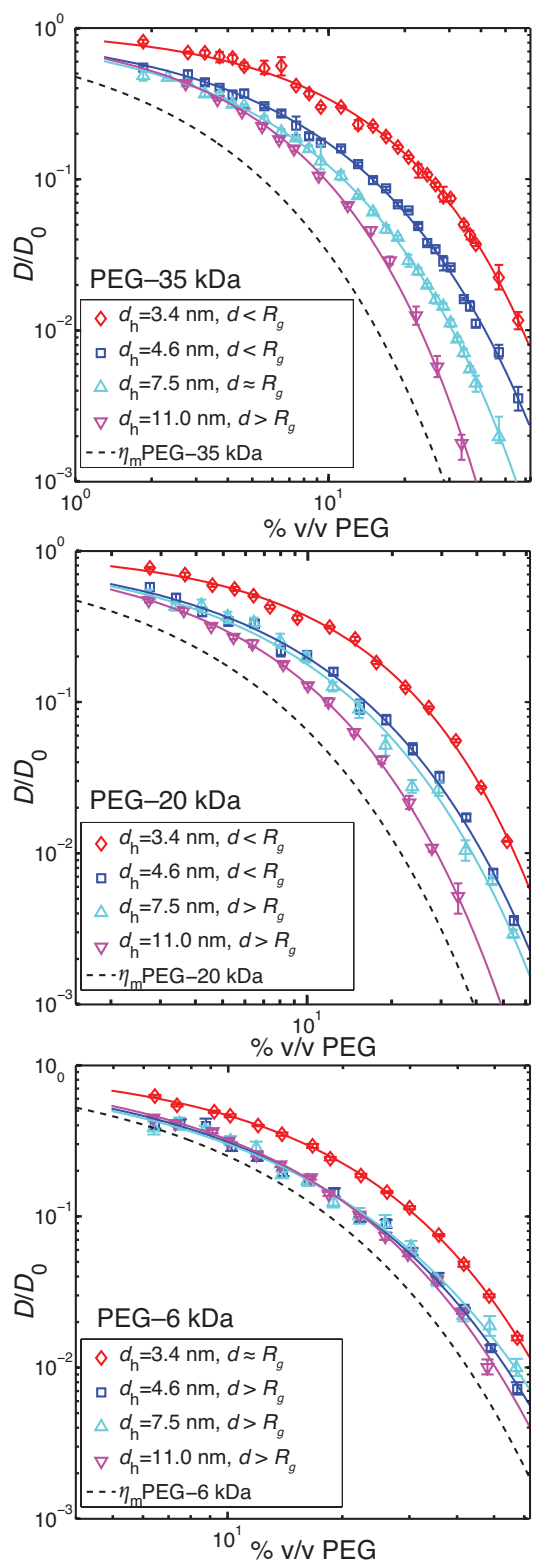

Figure 6.5: Diffusion coefficients of nanoparticles in PEG as a function of polymer volume fraction, sorted by polymer molecular weight. Solid lines are fits to stretched exponential curves; these curves begin at the overlap concentration. The black-dotted line is the Stokes-Einstein result $D / D_{0}=\eta_{0} / \eta_{\mathrm{m}}$. 


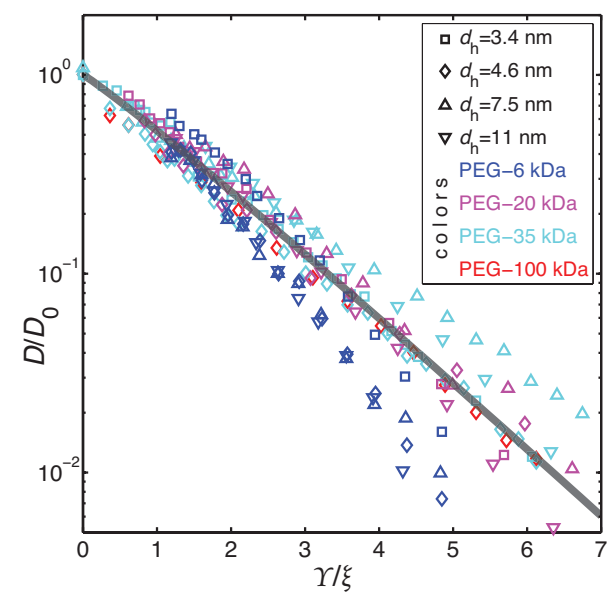

Figure 6.6: Master curve $\left(D / D_{0}\right) \sim \exp \left[-a(\Upsilon / \xi)^{b}\right]$, where $a=0.65$ and $\boldsymbol{b}=1.1$. For $d<R_{g}$, parameter $\Upsilon=d$. For $d>R_{g}, \Upsilon=R_{g}$. For comparison, plots where $\Upsilon=d$ for all data points and $\Upsilon=R_{g}$ for all data points can be found in SI section S6.6.

Cai et al. provided more tangible arguments for the behavior of the diffusion coefficients when $d>R_{g}$. They unified the scaling predications for diffusion coefficients for intermediate sized particles $(\xi<d<a)$ at both $d<R_{g}$ and $d>R_{g}$. The crossover length scale should manifest itself in a plot of $\left(D / D_{\text {un }}\right)$ against $d / R_{g}$, where $D_{\text {un }}$ is the diffusion coefficient of particles if the solution would be "unentangled", and where $R_{g}=R_{g}\left(\varphi^{*}\right) \varphi^{-1 / 8}$. For intermediate-size particles, $\left(D / D_{\text {un }}\right) \sim\left(d / R_{g}\right)^{-2}$ at $d / R_{g}<1$ and $\left(D / D_{\text {un }}\right) \sim 1$ at $d / R_{g}>1$. The crossover length scale can therefore be determined as the point where the data starts to plateau in this depiction, as shown in Figure 6.7.

As input for the viscosity of "unentangled" solutions we used the viscosity of PEG-6 kDa, which cannot be considered entangled in this concentration range. This results in a plot in which points of each curve group together and follow the scaling predictions rather convincingly, although we should take into account that the $D_{\text {un }}$ is only estimated and that the error bar on each data point does not include the error of this estimation. Nonetheless, the data level off at $d>R_{g}$, confirming that this is the crossover length scale. 


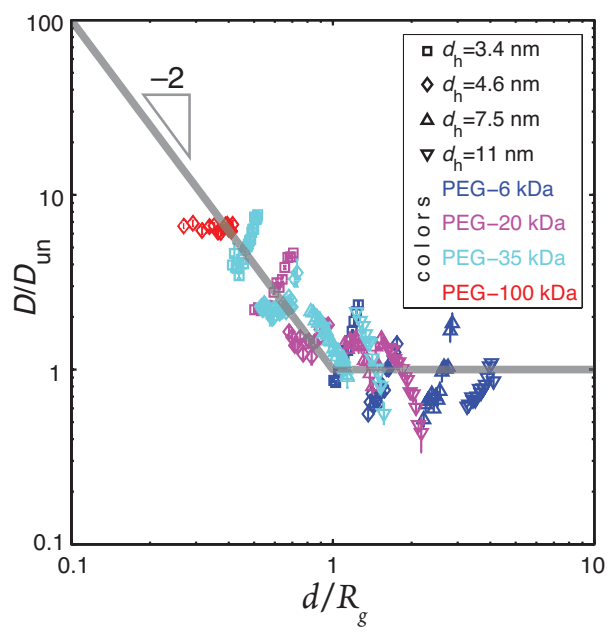

Figure 6.7: Plot of $\left(D / D_{\text {un }}\right)$ against $d / R_{g}$, where $D_{\text {un }}$ is the diffusion coefficient of particles if the solution would be unentangled, and $R_{g}=R_{g}\left(\varphi^{*}\right) \varphi^{-1 / 8}$. For $d / R_{g}<1,\left(D / D_{\text {un }}\right) \sim\left(d / R_{g}\right)^{-2}$ and for $d / R_{g}>1$, $\left(D / D_{\text {un }}\right) \sim 1$ for intermediate-size particles.

Scaling behavior of intermediate-size particles smaller than polymer coils. Lastly, we investigate the details of the scaling behavior of intermediate-size particles $(\xi<d<a)$ that are smaller than the polymer coils $\left(d<R_{g}\right)$ In Figure 6.8, the corresponding data points have been plotted as a function of $\varphi / \varphi_{\xi=d}$, where $\varphi_{\xi=d}$ is the crossover point into the $\xi<d<a$ regime.

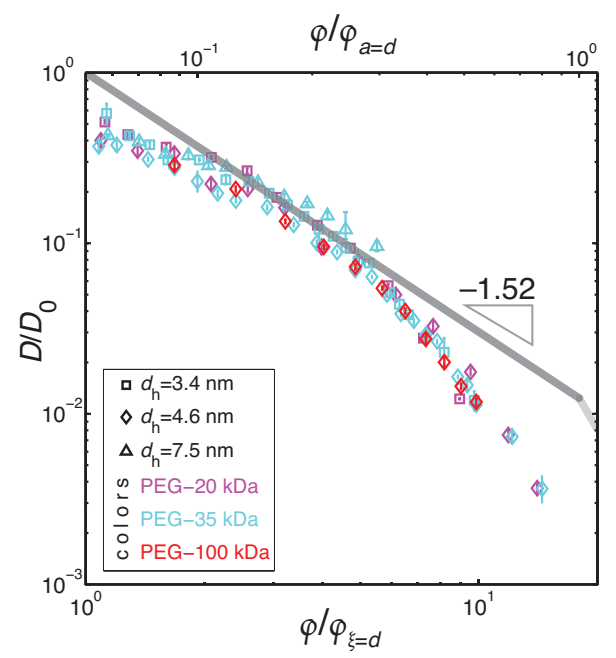

Figure 6.8: Normalized nanoparticle diffusion coefficients $D / D_{0}$ in the $\xi<d<a\left(D \sim \varphi^{-1.52}\right)$ regime for all data points for which $d<R_{g}$. 
All data points overlap in this depiction, which is not the case if the $d>R_{g}$ data are included (This can be seen in Supporting Information section S6.7 where all data have been plotted together in a similar fashion). The scaling prediction has intrinsic difficulties describing the data to within the experimental error, as the transition between different regimes is always smooth in reality. Nonetheless, the -1.52 scaling exponent gives an adequate prediction of the decrease of diffusion coefficient with polymer volume fraction in this regime.

\subsection{Conclusions}

The use of internally ${ }^{19} \mathrm{~F}$-labeled dendritic nanoparticles $\left(d_{\mathrm{h}}=3.4-11.0 \mathrm{~nm}\right)$ with inert surface chemistries allowed for background free ${ }^{19} \mathrm{~F}$ NMR self-diffusion measurements in semidilute solutions of PEG $\left(M_{\mathrm{w}}=6,20,35\right.$ and $\left.100 \mathrm{kDa}\right)$. The diffusion coefficients of these nanoparticles decreased in a stretched-exponential manner with PEG volume fraction. In case $d<R_{g}$ the diffusion coefficients of particles of a fixed diameter $d$ are independent of the PEG molecular weight. In this regime, diffusion curves are described by Cukier's model $D \sim \exp \left(\varphi^{3 / 4}\right)$. Although in none of the measured systems the particles fully experienced the macroviscosity, a crossover point at $d=R_{g}$ could be visualized most convincingly by means of scaling arguments presented by Cai et al. The same model made detailed scaling predictions for the diffusion coefficients of "intermediate size" particles $\left(\xi<d<a\right.$ at $\left.D<R_{g}\right)$ as a function of polymer volume fraction. We have shown that these predictions are accurate, and break down exactly at the crossover point $d=R_{g}$.

\section{Supporting Information}

The following material is available free of charge via the Internet at http://doi.org/10.1021/acs.macromol.5b01530: S6.1. Synthesis and characterization of ${ }^{19} \mathrm{~F}$ labeled PPI based nanoparticles.

All other material is included below: Table S6.1. Overview of the features and physical properties of the four different ${ }^{19} \mathrm{~F}$ labeled nanoparticles. S6.2. Relaxation times of poly(ethylene glycol) solutions in water. S6.3. Length scales in solutions of poly(ethylene glycol) in water. S6.4. Macroscopic zero-shear viscosities $\eta_{\mathrm{m}}$ of poly(ethylene glycol) solutions in water at different polymer volume fractions. S6.5. Ratio $D / D\left(\eta_{\mathrm{m}}\right)$ for all data points. S6.6. Plots of $\left(D / D_{0}\right)$ against $R_{g} / \xi$ and $d / \xi$ for all data points. S6.7. Effect of normalization to $\varphi_{\xi=d}$ at both $d>R_{g}$ and $d<R_{g}$.

\section{Acknowledgments}

We thank Alex van der Rest and Max Brouwer for valuable experimental contributions. This research received funding from the Netherlands Organization for Scientific Research (NWO) in the framework of the Technology Area COAST. 


\begin{tabular}{|c|c|c|c|c|}
\hline Nanoparticle & $\begin{array}{l}\text { G1PPI }\left(F_{3^{-}}\right. \\
\text {PEG })_{4}\end{array}$ & $\begin{array}{l}\text { G3PPI }\left(F_{3-}-\right. \\
\text { PEG })_{16}\end{array}$ & $\begin{array}{l}\text { G5PPI }\left(F_{3^{-}}\right. \\
\text {PEG })_{64}\end{array}$ & “G6”( $\left.F_{9}-P E G\right){ }_{192}$ \\
\hline Type of dendritic core & G1PPI- $\left(\mathrm{NH}_{2}\right)_{4}$ & G3PPI- $\left(\mathrm{NH}_{2}\right)_{16}$ & G5PPI- $\left(\mathrm{NH}_{2}\right)_{64}$ & 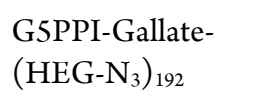 \\
\hline Number of exterior mPEG groups & 4 & 16 & 64 & 192 \\
\hline \multirow[t]{2}{*}{ Type of coronal shielding group } & mPEG-543 & mPEG-543 & mPEG-543 & mPEG-750 \\
\hline & (discrete $\left.\boldsymbol{M}_{\mathrm{w}}\right)$ & $\left(\right.$ discrete $\left.\boldsymbol{M}_{\mathrm{w}}\right)$ & $\left(\right.$ discrete $\left.\boldsymbol{M}_{\mathrm{w}}\right)$ & (distribution in $\boldsymbol{M}_{\mathrm{w}}$ ) \\
\hline Type of ${ }^{19} \mathrm{~F}$ group & $-\mathrm{CF}_{3}$ & $-\mathrm{CF}_{3}$ & $-\mathrm{CF}_{3}$ & $-\mathrm{C}\left(\mathrm{CF}_{3}\right)_{3}$ \\
\hline $\mathrm{wt} \%{ }^{19} \mathrm{~F}$ & 7.2 & 7.0 & 6.9 & 11.6 \\
\hline Molecular weight $(\mathrm{kDa})$ & 3.158 & 13.043 & 52.592 & 282.500 \\
\hline Hydrodynamic diameter $\left(d_{\mathrm{h}}\right)$ & $3.4 \pm 0.3$ & $4.6 \pm 0.2$ & $7.5 \pm 0.3$ & $11.0 \pm 0.5$ \\
\hline$T_{1}(\mathrm{~ms})$ of $\mathrm{PEG}^{-1} \mathrm{H}(-3.7 \mathrm{ppm})$ at $7.0 \mathrm{~T}$ & $633 \pm 20$ & $538 \pm 18$ & $529 \pm 23$ & $432 \pm 33$ \\
\hline$T_{2}(\mathrm{~ms})$ of $\mathrm{PEG}-{ }^{1} \mathrm{H}(-3.7 \mathrm{ppm})$ at $7.0 \mathrm{~T}$ & $589 \pm 25$ & $505 \pm 28$ & $375 \pm 30$ & $294 \pm 28$ \\
\hline$T_{1}(\mathrm{~ms})$ of ${ }^{19} \mathrm{~F}$ labels $(-64 \mathrm{ppm})$ at $7.0 \mathrm{~T}$ & $620 \pm 12$ & $561 \pm 11$ & $412 \pm 15$ & $543 \pm 24$ \\
\hline$T_{2}(\mathrm{~ms})$ of ${ }^{19} \mathrm{~F}$ labels $(-64 \mathrm{ppm})$ at $7.0 \mathrm{~T}$ & $400 \pm 10$ & $240 \pm 12$ & $129 \pm 12$ & $82 \pm 7$ \\
\hline
\end{tabular}

Table S6.1: Overview of the features and physical properties of the four different ${ }^{19} \mathrm{~F}$ labeled dendritic nanoparticles. Hydrodynamic diameters were calculated from self-diffusion coefficients in water at $298 \mathrm{~K}$ through the Stokes-Einstein equation. 


\section{S6.2 Relaxation times of poly(ethylene glycol) solutions in water}

\section{Kuhn length $b$}

$b=C_{\infty} l / \cos (\theta / 2)$, where $C_{\infty}=5.5$ (Flory characteristic ratio) (Fetters, Lohse, Richter, \& Witten, $1994), l=0.146 \mathrm{~nm}$ (average bond length in (C-C-O- ${ }_{n}$ backbone) (Mark \& Flory, 1965), $\theta=70^{\circ}$ (average backbone bond angle) (Mark \& Flory, 1965), so Kuhn length $b \cong 0.98 \mathrm{~nm}$.

\section{Kuhn monomer relaxation time $\tau_{0}$}

$\tau_{0} \cong \frac{\eta_{s} b^{3}}{k_{B} T}$

$\eta_{s}=1 \mathrm{mPa} \mathrm{s}$ (viscosity of water at room temperature), $b \cong 0.98 \mathrm{~nm}$ (PEG Kuhn length), $k_{B}$ the Boltzmann constant, $T=293 \mathrm{~K}$ (room temperature), so $\tau_{0} \cong 0.2 \mathrm{~ns}$.

\section{Kuhn monomers per entanglement strand in the melt $N_{e}(1)$}

$N_{e}(1)=(a(1) / b)^{2}$, where $a(1)=3.73 \mathrm{~nm}$ (tube diameter in the melt) (Fetters, Lohse, \& Colby, 2007) and $b \cong 0.98 \mathrm{~nm}$ (Kuhn length), so $N_{e}(1) \cong 14$.

\section{Reptation time $\tau_{\text {rep }}$}

$\tau_{\text {rep }} \cong \tau_{0} \frac{N^{3}}{N_{e}(1)} \phi^{3(1-v) /(3 v-1)} \cong \tau_{0} \frac{N^{3}}{N_{e}(1)} \phi^{3 / 2}(v=3 / 5$ in good solvent $)$

$N_{e}(1)=14$ (monomers per entanglement in the melt), $\phi \cong 0.60$ (highest polymer volume fraction used in this study), $M_{0}=M_{\mathrm{e}} / N_{\mathrm{e}}=2000 / 14=1.4 \times 10^{2} \mathrm{~g} / \mathrm{mol}$ (Kuhn monomer molar mass) (Fetters et al., 2007), so $N(6 \mathrm{kDa})=6 \times 10^{3} / 1.4 \times 10^{2}=43, N(20 \mathrm{kDa})=20 \times 10^{3} / 1.4 \times 10^{2}=1.4 \times 10^{2}$, $N(35 \mathrm{kDa})=35 \times 10^{3} / 1.4 \times 10^{2}=2.5 \times 10^{2}$ and $N(100 \mathrm{kDa})=100 \times 10^{3} / 1.4 \times 10^{2}=7.0 \times 10^{2}$ (number of Kuhn monomers per chain). Conclusion: $\tau_{\text {rep }}(6 \mathrm{kDa}) \cong 0.5 \mu \mathrm{s}, \tau_{\text {rep }}(20 \mathrm{kDa}) \cong$ $0.02 \mathrm{~ms}, \tau_{\text {rep }}(35 \mathrm{kDa}) \cong 0.1 \mathrm{~ms}$ and $\tau_{\text {rep }}(100 \mathrm{kDa}) \cong 2 \mathrm{~ms}$.

In the case of $6 \mathrm{kDa}$ PEG, the number of entanglements formed per strand is $N(6 \mathrm{kDa}) / N_{e}(1)=43 / 14=3$. Because this number is $\ll 10$, PEG $6 \mathrm{kDa}$ cannot be considered to entangle.

For PEG weights up to $100 \mathrm{kDa}$, the slowest dynamical process is apparently still faster than $10 \mathrm{~ms}$, which is the onset of the NMR diffusometry time scale. We therefore measure terminal selfdiffusion coefficients at the experimental time scale $(100 \mathrm{~ms})$ even in case the entanglements would influence particle self-diffusion. 


\section{S6.3 Length scales in solutions of poly(ethylene glycol) in water}

\section{Overlap concentration, correlation length, radius of gyration}

Chain overlap concentration $\phi^{*}$ is given by $\phi^{*}=\frac{M_{w}}{\frac{4}{3} \pi\left(R_{g}^{\phi^{*}}\right)^{3} \rho N_{A}}\left(\right.$ in $\left.\mathrm{g} / \mathrm{m}^{3}\right)$, where $M$ is molecular weight, $R_{g}^{\phi^{*}}=0.215 \times 10^{-10} M_{w}^{7 / 12}$ (radius of gyration at the overlap concentration: $R_{g}^{\phi^{*}}(6 \mathrm{kDa})=3.4 \mathrm{~nm}, R_{g}^{\phi^{*}}(20 \mathrm{kDa})=6.9 \mathrm{~nm}, R_{g}^{\phi^{*}}(35 \mathrm{kDa})=9.6 \mathrm{~nm}$ and $\left.R_{g}^{\phi^{*}}(100 \mathrm{kDa})=18 \mathrm{~nm}\right)$ (Devanand \& Selser, 1991), $\rho=1081 \mathrm{~kg} / \mathrm{m}^{3}$ (density) (Fetters et al., 2007) and $N_{A}$ Avogadro's number $\left(\mathrm{mol}^{-1}\right)$. This results in $\phi^{*}(6 \mathrm{kDa})=0.054, \phi^{*}(20 \mathrm{kDa})=0.022, \phi^{*}(35 \mathrm{kDa})=0.014$ and $\phi^{*}(100 \mathrm{kDa})=0.0066$.

Above the overlap concentration, correlation length/blob size decreases as $\xi(\phi)=R_{g}^{\phi^{*}}\left(\frac{\phi}{\phi^{*}}\right)^{-3 / 4}$ while the radius of gyration decreases as $R_{g}(\phi)=R_{g}^{\phi^{*}}\left(\frac{\phi}{\phi^{*}}\right)^{-1 / 8}$.

\section{Entanglement volume fraction, tube diameter}

Entanglement volume fraction $\phi_{e}=\left(\frac{N_{e}(1)}{N}\right)^{3 / 4} . N$ is the number of Kuhn monomers per chain $\left(N(6 \mathrm{kDa})=43, N(20 \mathrm{kDa})=1.4 \times 10^{2}, N(35 \mathrm{kDa})=2.5 \times 10^{2}\right.$ and $N(100 \mathrm{kDa})=7.0 \times 10^{2}$, cf. S6.2). The number of entanglements per entanglement strand in the melt $N_{e}(1)=14$ (cf. S6.2). PEG $6 \mathrm{kDa}$ will therefore never truly entangle. This results in $\phi_{e}(20 \mathrm{kDa})=0.17$, $\phi_{e}(35 \mathrm{kDa})=0.11$ and $\phi_{e}(100 \mathrm{kDa})=0.051$.

Above the entanglement concentration, tube diameter $a(\phi)=a(1) \phi^{-3 / 4}$, where the tube diameter in the melt $a(1)=3.73 \mathrm{~nm}$ (Fetters et al., 2007). 
S6.4 Macroscopic zero-shear viscosities $\eta_{\mathrm{m}}$ of poly(ethylene glycol) solutions in water at different polymer volume fractions

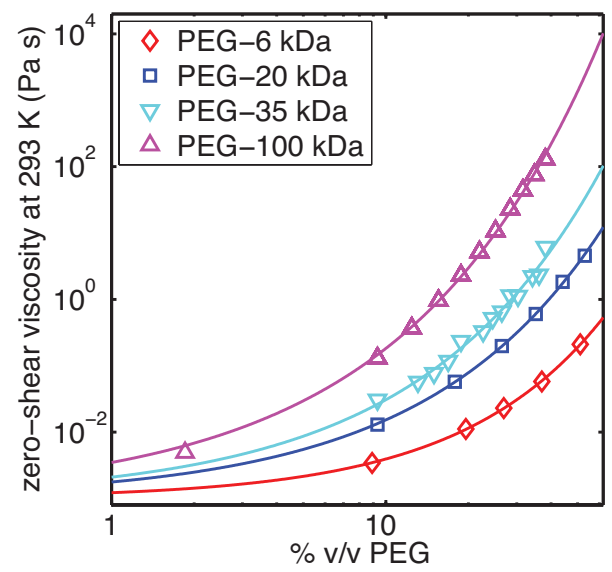

Figure S6.1: Macroscopic zero-shear viscosities $\eta_{\mathrm{m}}$ of PEG solutions as a function of volume fraction. The data points were fitted with stretched exponential functions with the condition that the viscosity of a $0 \% \mathrm{v} / \mathrm{v}$ solution has the viscosity of water at $293 \mathrm{~K}$ ( $1 \mathrm{mPa} \mathrm{s})$. 
S6.5 Ratio $D / D\left(\eta_{\mathrm{m}}\right)$ for all data points
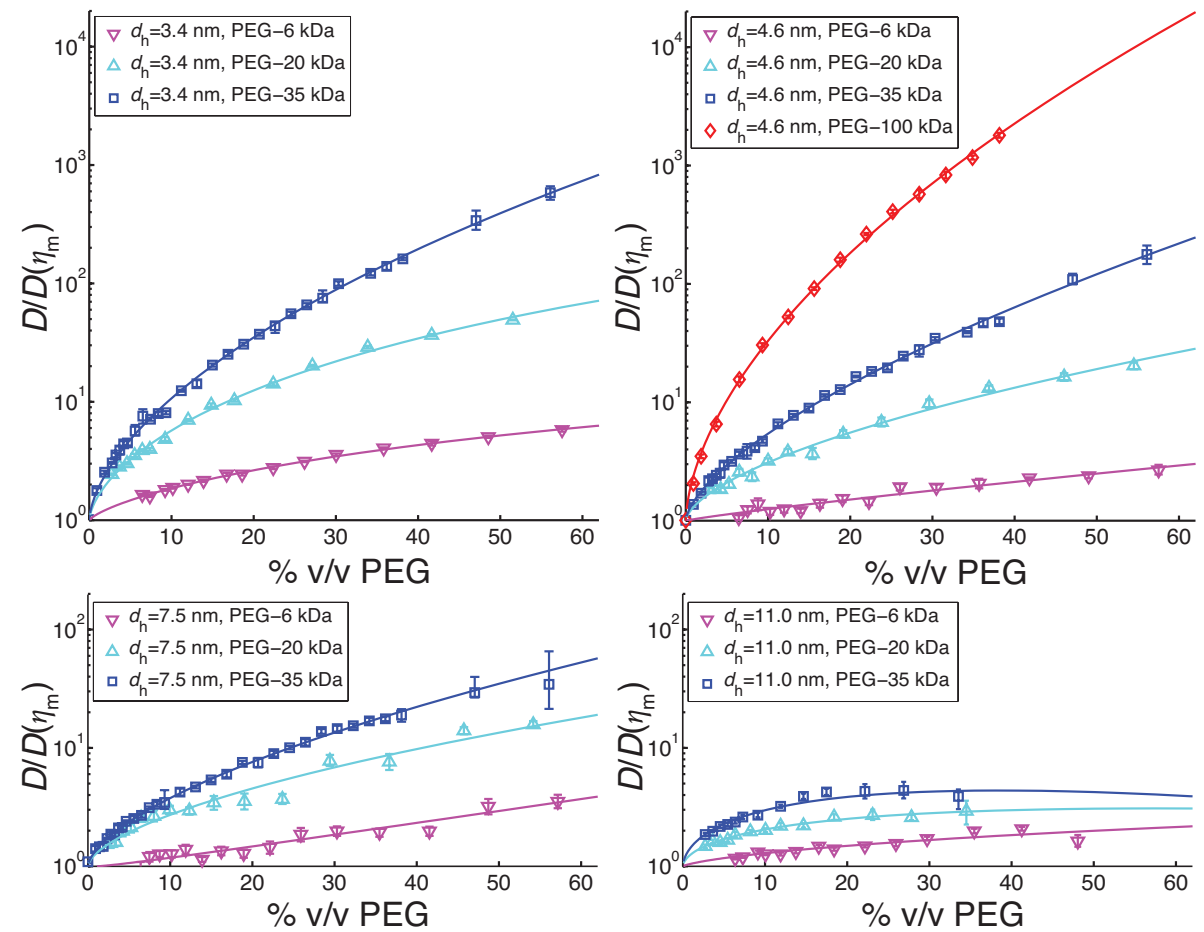

Figure S6.2: $D / D\left(\eta_{\mathrm{m}}\right)$ ratio for all data points, where $D\left(\eta_{\mathrm{m}}\right)$ follows from the Stokes-Einstein relation. 


\section{S6.6 Plots of $\left(D / D_{0}\right)$ against $R_{g} / \xi$ and $d / \xi$ for all data points}
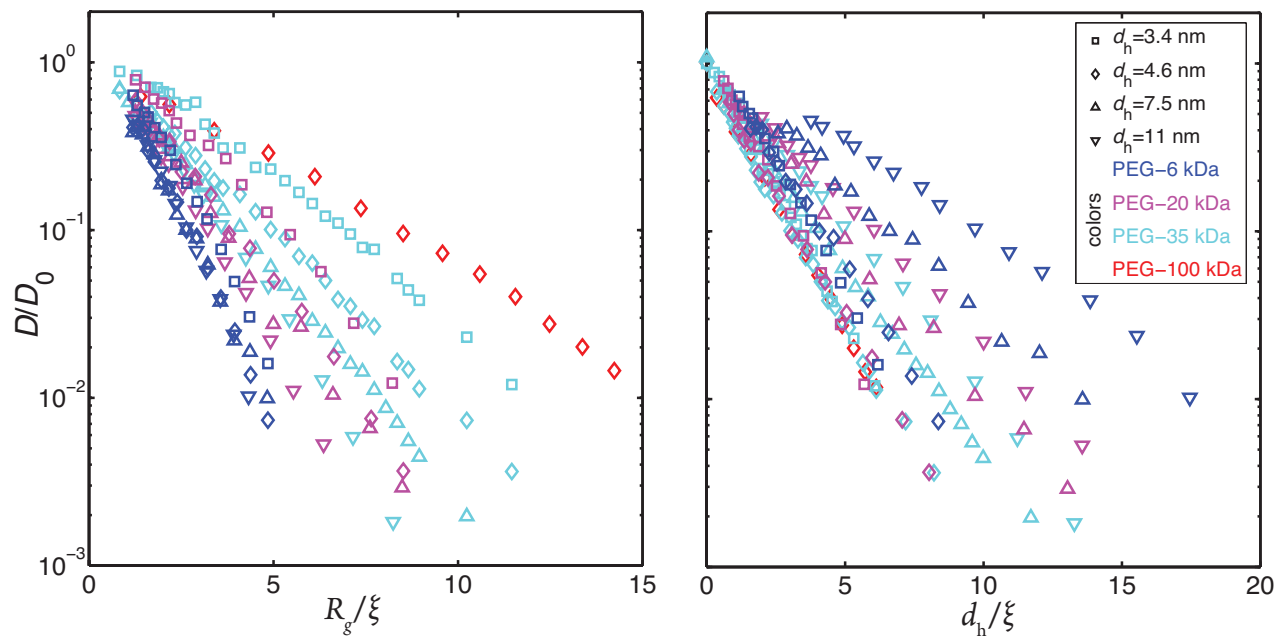

Figure S6.3: Plots of $\left(D / D_{0}\right)$ against $R_{g} / \xi$ and $d / \xi$ for all data points. Here, $\xi(\phi)=R_{g}^{\phi^{*}}\left(\frac{\phi}{\phi^{*}}\right)^{-3 / 4}$ and $R_{g}(\phi)=R_{g}^{\phi^{*}}\left(\frac{\phi}{\phi^{*}}\right)^{-1 / 8}$, where $\phi$ is polymer volume fraction.

S6.7 Effect of normalization to $\varphi_{\xi=d}$ at both $d>R_{g}$ and $d<R_{g}$
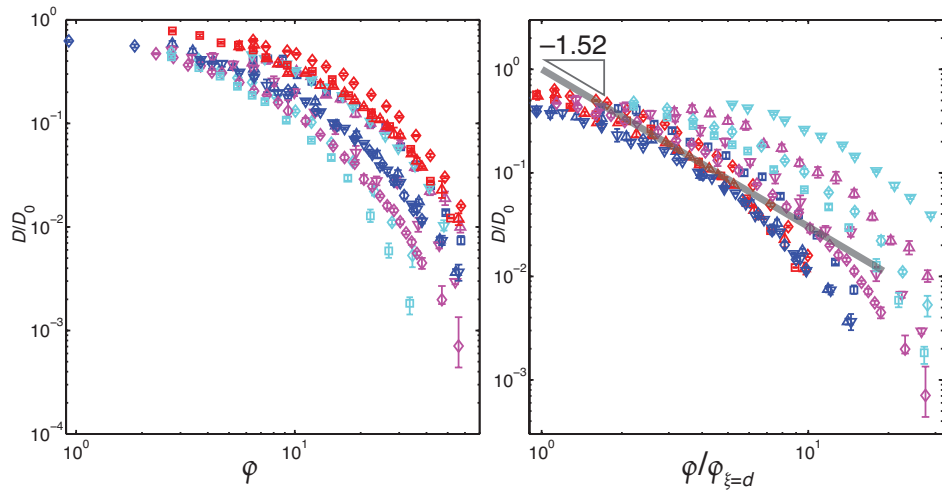

$d_{\mathrm{h}}=3.4 \mathrm{~nm}, \mathrm{PEG}-6 \mathrm{kDa}$ $d_{\mathrm{h}}=4.6 \mathrm{~nm}, \mathrm{PEG}-6 \mathrm{kDa}$ $d_{\mathrm{h}}=7.5 \mathrm{~nm}, \mathrm{PEG}-6 \mathrm{kDa}$ $d_{\mathrm{h}}=11 \mathrm{~nm}, \mathrm{PEG}-6 \mathrm{kDa}$ $d_{\mathrm{h}}=3.4 \mathrm{~nm}, \mathrm{PEG}-20 \mathrm{kDa}$ $d_{\mathrm{h}}=4.6 \mathrm{~nm}, \mathrm{PEG}-20 \mathrm{kDa}$ $d_{\mathrm{h}}=7.5 \mathrm{~nm}, \mathrm{PEG}-20 \mathrm{kDa}$ $d_{\mathrm{h}}=11 \mathrm{~nm}, \mathrm{PEG}-20 \mathrm{kDa}$ $d_{\mathrm{h}}=3.4 \mathrm{~nm}, \mathrm{PEG}-35 \mathrm{kDa}$ $d_{\mathrm{h}}=4.6 \mathrm{~nm}, \mathrm{PEG}-35 \mathrm{kDa}$ $d_{\mathrm{h}}=7.5 \mathrm{~nm}, \mathrm{PEG}-35 \mathrm{kDa}$ $d_{\mathrm{h}}=11 \mathrm{~nm}, \mathrm{PEG}-35 \mathrm{kDa}$ $d_{\mathrm{h}}=4.6 \mathrm{~nm}, \mathrm{PEG}-100 \mathrm{kDa}$

Figure S6.4: In this figure, the effect of normalization of the data points to the crossover point into the intermediate regime $\boldsymbol{\phi}_{\xi=d}$ is shown. For particles larger than $R_{g}^{\phi^{*}}$ (the polymer coil size at the overlap concentration), we use a trivial backprediction of $\xi$ to calculate an "apparent" $\boldsymbol{\varphi}_{\xi=d}$. Careful comparison of the left and right panels leads to the conclusion that this normalization leads to overlap of those data points belonging to intermediate particles smaller than the polymer coil size only $\left(\xi<d<a\right.$ and $\left.d<R_{g}\right)$. Only these points are shown in Figure 6.8 in the main text. 


\section{References}

Altenberger, A. R., Tirrell, M., \& Dahler, J. S. (1986). Hydrodynamic screening and particle dynamics in porous media, semidilute polymer solutions and polymer gels. The Journal of Chemical Physics, 84(9), 5122-5130. http://doi.org/10.1063/1.450665

Brinkman, H. C. (1949). A calculation of the viscous force exerted by a flowing fluid on a dense swarm of particles. Applied Scientific Research, 1(1), 27-34. http://doi.org/10.1007/BF02120313

Brochard Wyart, F., \& de Gennes, P. G. (2000). Viscosity at small scales in polymer melts. European Physical Journal E, 1(1), 93-97. http://doi.org/10.1007/s101890050011

Cai, L.-H., Panyukov, S., \& Rubinstein, M. (2011). Mobility of Nonsticky Nanoparticles in Polymer Liquids. Macromolecules, 44(19), 7853-7863. http://doi.org/10.1021/ma201583q

Cai, L.-H., Panyukov, S., \& Rubinstein, M. (2015). Hopping Diffusion of Nanoparticles in Polymer Matrices. Macromolecules, 48(3), 847-862. http://doi.org/10.1021/ma501608x

Callaghan, P. T. (2011). Translational Dynamics \& Magnetic Resonance. New York: Oxford University Press.

Colby, R. H., \& Rubinstein, M. (1990). Two-parameter scaling for polymers in $\Theta$ solvents. Macromolecules, 23(10), 2753-2757. http://doi.org/10.1021/ma00212a028

Cukier, R. I. (1984). Diffusion of Brownian spheres in semidilute polymer solutions. Macromolecules, 17(2), 252-255. http://doi.org/10.1021/ma00132a023

Davison, A. C., \& Hinkley, D. V. (1997). Bootstrap Methods and Their Application. Cambridge University Press.

de Gennes, P. G. (1976). Dynamics of entangled polymer solutions. I. The Rouse model. Macromolecules, 9(4), 587-593. http://doi.org/10.1021/ma60052a011

de Gennes, P. G. (1979). Scaling Concepts in Polymer Physics. Cornell University Press.

de Kort, D. W., van Duynhoven, J. P. M., Hoeben, F. J. M., Janssen, H. M., \& Van As, H. (2014). NMR nanoparticle diffusometry in hydrogels: enhancing sensitivity and selectivity. Analytical Chemistry, 86(18), 9229-9235. http://doi.org/10.1021/ac502211q

Debye, P., \& Bueche, A. M. (1948). Intrinsic Viscosity, Diffusion, and Sedimentation Rate of Polymers in Solution. The Journal of Chemical Physics, 16(6), 573. http://doi.org/10.1063/1.1746948

Devanand, K., \& Selser, J. C. (1991). Asymptotic behavior and long-range interactions in aqueous solutions of poly(ethylene oxide). Macromolecules, 24(22), 5943-5947. http://doi.org/10.1021/ma00022a008

Fetters, L. J., Lohse, D. J., \& Colby, R. H. (2007). Chain Dimensions and Entanglement Spacings. In J. E. Mark (Ed.), Physical Properties of Polymers Handbook (pp. 447-454). New York, NY: Springer New York. http://doi.org/10.1007/978-0-387-69002-5_25

Fetters, L. J., Lohse, D. J., Richter, D., \& Witten, T. A. (1994). Connection between polymer molecular weight, density, chain dimensions, and melt viscoelastic properties. Macromolecules, 27(17), 46394647. http://doi.org/10.1021/ma00095a001

Grabowski, C. A., \& Mukhopadhyay, A. (2014). Size Effect of Nanoparticle Diffusion in a Polymer Melt. Macromolecules, 47(20), 7238-7242. http://doi.org/10.1021/ma501670u

Hedden, R. C., \& Bauer, B. J. (2003). Structure and Dimensions of PAMAM/PEG Dendrimer-Star Polymers. Macromolecules, 36(6), 1829-1835. http://doi.org/10.1021/ma025752n

Hołyst, R., Bielejewska, A., Szymański, J., Wilk, A., Patkowski, A., Gapiński, J., et al. (2009). Scaling form of viscosity at all length-scales in poly(ethylene glycol) solutions studied by fluorescence correlation spectroscopy and capillary electrophoresis. Physical Chemistry and Chemical Physics : PCCP, 11(40), 9025-9032. http://doi.org/10.1039/b908386c

Höfling, F., \& Franosch, T. (2013). Anomalous transport in the crowded world of biological cells. Reports on Progress in Physics, 76(4), 046602. http://doi.org/10.1088/0034-4885/76/4/046602

Kalwarczyk, T., Sozanski, K., Ochab-Marcinek, A., Szymański, J., Tabaka, M., Hou, S., \& Hołyst, R. (2015). Motion of nanoprobes in complex liquids within the framework of the length-scale dependent viscosity 
model. Advances in Colloid and Interface Science, 223, 55-63. http://doi.org/10.1016/j.cis.2015.06.007

Kohli, I., \& Mukhopadhyay, A. (2012). Diffusion of Nanoparticles in Semidilute Polymer Solutions: Effect of Different Length Scales. Macromolecules, 45(15), 6143-6149. http://doi.org/10.1021/ma301237r

Kumar, S. K., Jouault, N., Benicewicz, B., \& Neely, T. (2013). Nanocomposites with Polymer Grafted Nanoparticles. Macromolecules, 46(9), 3199-3214. http://doi.org/10.1021/ma4001385

Langevin, D., \& Rondelez, F. (1978). Sedimentation of large colloidal particles through semidilute polymer solutions. Polymer, 19(8), 875-882. http://doi.org/10.1016/0032-3861(78)90191-X

Le Guillou, J., \& Zinn-Justin, J. (1977). Critical Exponents for the n-Vector Model in Three Dimensions from Field Theory. Physical Review Letters, 39(2), 95-98. http://doi.org/10.1103/PhysRevLett.39.95

Mark, J. E., \& Flory, P. J. (1965). The configuration of the polyoxyethylene chain. Journal of the American Chemical Society, 87(7), 1415-1423. http://doi.org/10.1021/ja01085a001

Mason, T. G., \& Weitz, D. A. (1995). Optical Measurements of Frequency-Dependent Linear Viscoelastic Moduli of Complex Fluids. Physical Review Letters, 74(7), 1250-1253. http://doi.org/10.1103/PhysRevLett.74.1250

Meyvis, T. K. L., De Smedt, S. C., Van Oostveldt, P., \& Demeester, J. (1999). Fluorescence Recovery After Photobleaching: A Versatile Tool for Mobility and Interaction Measurements in Pharmaceutical Research. Pharmaceutical Research, 16(8), 1153-1162. http://doi.org/10.1023/A:1011924909138

Novikov, D. S., Fieremans, E., Jensen, J. H., \& Helpern, J. A. (2011). Random walks with barriers. Nature Physics, 7(6), 508-514. http://doi.org/10.1038/nphys1936

Ochab-Marcinek, A., \& Holyst, R. (2011). Scale-dependent diffusion of spheres in solutions of flexible and rigid polymers : mean square displacement and autocorrelation function for FCS and DLS measurements. Soft Matter, 7(16), 7366-7374. http://doi.org/10.1039/C1SM05217A

Rathgeber, S., Beauvisage, H.-J., Chevreau, H., Willenbacher, N., \& Oelschlaeger, C. (2009). Microrheology with Fluorescence Correlation Spectroscopy. Langmuir, 25(11), 6368-6376. http://doi.org/10.1021/la804170k

Stejskal, E. O., \& Tanner, J. E. (1965). Spin diffusion measurements: Spin echoes in the presence of a timedependent field gradient. The Journal of Chemical Physics, 42, 288-292. http://doi.org/10.1063/1.1695690

Topp, A., Bauer, B. J., Klimash, J. W., Spindler, R., Tomalia, D. A., \& Amis, E. J. (1999). Probing the Location of the Terminal Groups of Dendrimers in Dilute Solution. Macromolecules, 32(21), 7226-7231. http://doi.org/10.1021/ma990125s

Valentine, M., Kaplan, P., Thota, D., Crocker, J., Gisler, T., Prud'homme, R., et al. (2001). Investigating the microenvironments of inhomogeneous soft materials with multiple particle tracking. Physical Review E, 64(6), 061506. http://doi.org/10.1103/PhysRevE.64.061506

van Resandt, R. W. W., Vogel, R. H., \& Provencher, S. W. (1982). Double beam fluorescence lifetime spectrometer with subnanosecond resolution: Application to aqueous tryptophan. Review of Scientific Instruments, 53(9), 1392. http://doi.org/10.1063/1.1137173

Vogel, R. H. (1988). SplMod Users Manual, Version 3. Heidelberg.

Ziębacz, N., Wieczorek, S. A., Kalwarczyk, T., Fiałkowski, M., \& Hołyst, R. (2011). Crossover regime for the diffusion of nanoparticles in polyethylene glycol solutions: influence of the depletion layer. Soft Matter, 7(16), 7181-7186. http://doi.org/10.1039/COSM01357A 



\title{
Yielding and flow of cellulose microfibril dispersions in the presence of charged polymer
}

\begin{abstract}
Shear flow of microfibrillated cellulose dispersions is still not wholly understood as a consequence of their multi-length-scale heterogeneity. We added carboxymethyl cellulose, a charged polymer that makes cellulose microfibril dispersions more homogeneous at the submicron and macro scales. We then compared the yielding and flow behavior of these dispersions to that of typical thixotropic yield-stress fluids. Despite of the apparent homogeneity of the dispersions, their flow velocity profiles in cone-plate geometry, as measured by rheo-MRI velocimetry, differ strongly from those observed for typical thixotropic model systems: the viscosity across the gap is not uniform, despite a flat stress field across the gap. We describe these velocity profiles with a nonlocal model, and attribute the non-locality to persistent micron-scale structural heterogeneity.
\end{abstract}

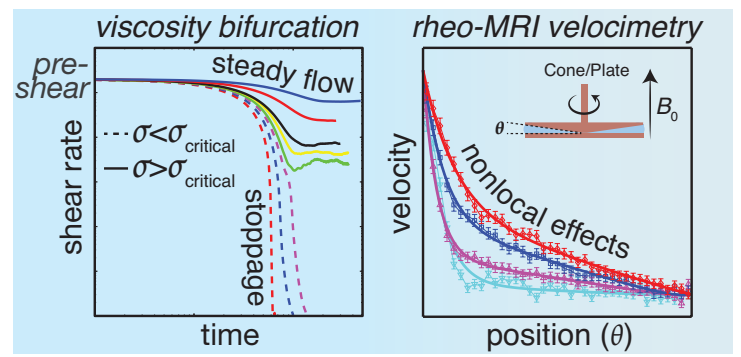

Daan W. de Kort, Sandra J. Veen, Henk Van As, Daniel Bonn, Krassimir P. Velikov, and John P.M. van Duynhoven, Soft Matter 2016, in press (DOI: 10.1039/c5sm02869h). 


\subsection{Introduction}

Dispersions of cellulose microfibrils (MFs) have received considerable attention as sustainable and natural materials with applications ranging from coatings and packaging materials to foods and cosmetics (Klemm et al., 2011). Their long, anisotropic shape allows the MFs to be used as scaffolding in polymer nanocomposites (Siró \& Plackett, 2010). For such composite gels, emphasis lies on their elastic properties, although their flow properties are also relevant, for instance for industrial processing and behavior during consumer use. A major obstacle in understanding flow is the compositional and structural heterogeneous nature of these dispersions. Cellulose MF dispersions are commonly prepared from native plant cellulose fibers by homogenization under very high pressures $(\sim \mathrm{MPa})$, separating the fibers into their constituent MFs (Chinga-Carrasco, 2011). Plant cellulose MFs have a high aspect ratio, leading to a low percolation threshold and gelation already at low concentrations (Lavoine, Desloges, Dufresne, \& Bras, 2012). Various strains of bacteria exist that excrete a chemically well-defined form of cellulose MFs. This bacterial cellulose (BC) is excreted as twisting ribbons, with diameters in the nanometers range and lengths of several micrometers. Bacterial cellulose has no functional groups other than hydroxyl groups (Klemm et al., 2011; Lin, Lopez-Sanchez, Li, \& Li, 2014). Although bacterial cellulose microfibrils (BCMF) are still polydisperse in size and width, we use them as model systems for microfibrillated plant cellulose because of their well-defined chemical properties.

Cellulose MFs have a strong tendency to aggregate due to Van der Waals and hydrogen bond driven interactions (Cousins, 1995) BCMFs have only a low $\zeta$-potential $(-7.5 \mathrm{mV})$, which is too low to stabilize them in aqueous suspensions, leading to their flocculation and sedimentation (Kuijk et al., 2013; Veen, Kuijk, Versluis, Husken, \& Velikov, 2014). Optical setups were used to measure floc sizes in MFPC dispersions under shear (Karppinen et al., 2012; Saarikoski, Saarinen, Salmela, \& Seppälä, 2012; Saarinen, Haavisto, Sorvari, Salmela, \& Seppälä, 2014). It was shown that, in a Taylor-Couette or concentric cylinders (CC) geometry at low MF concentrations (0.1$0.25 \mathrm{wt} \%)$ and low shear rates $\left(0.5 \mathrm{~s}^{-1}\right)$, the fibers accumulated into "log-rolling" cylinders aligned in the vorticity direction. This effect was not observed in more concentrated dispersions $(>1$ $w t \%)$, where the MFs remained more homogeneously distributed as flocs. The size of the flocs has been measured as a function of shear rate and was on the order of 0.1-1 mm, and typically decreased with shear rate. The presence of flocs lead to a depleted layer at the wall, because the centers of particles (flocs) can only approach the walls to within a distance equal to their radius (Barnes, 1995). This was visualized directly by Nechyporchuk et al., who used an optical setup to study flow of MF dispersions in cone-and-plate (CP) geometry (Nechyporchuk, Belgacem, \& Pignon, 2014). Under shear, a fast-moving water layer formed close to the cone due to the release of water from the bulk of the dispersion. Martoïa et al. used ultrasonic velocimetry to measure flow profiles within a CC geometry (Martoïa et al., 2015). They observed that the flow profile was banded and additionally that the flow profile was not uniform in the vorticity direction and 
strongly time dependent. Similar turbulent flow was observed in early MRI velocimetry studies, which was attributed to the strongly heterogeneous floc distribution (T. Q. Li, Seymour, Powell, \& McCarthy, 1994; Seymour, Maneval, McCarthy, McCarthy, \& Powell, 1993).

Addition of carboxymethyl cellulose (CMC), a negatively charged cellulose derivative, to BCMF dispersions and subsequent homogenization partly reversed flocculation. CMC is thought to interact with cellulose mainly through hydroxyl-mediated hydrogen bonding (Haigler, White, Brown, \& Cooper, 1982; Yamamoto \& Horn, 1994; Yan, Lindström, \& Christiernin, 2006). This results in stable gels with partly aligned MFs, depending on their concentration and the BCMF/CMC ratio (Veen et al., 2014). In these gels, the distribution of MFs over the available volume is homogeneous at the sub-micron length scale as observed by electron microscopy (Veen, Versluis, Kuijk, \& Velikov, 2015). These microstructural effects are accompanied by an increase in the magnitude of the elastic modulus. After loading the sample onto the rheometer, the elastic modulus gradually increased with time, suggesting that an MF network that has been broken down by shear can spontaneously recover in time (Veen et al., 2015). Although thixotropic behavior has been demonstrated for pristine BCMF dispersions (Chaouche \& Koch, 2001; Iotti, Gregersen, Moe, \& Lenes, 2010) it has not been clearly established for BCMF/CMC systems. The observations of the increase of the elastic modulus in time indicate that the rheology of $\mathrm{BCMF} / \mathrm{CMC}$ is similar to that of pristine BCMF dispersions, the difference being that the latter is much more heterogeneous than the former. In order to address the hypothesis that BCMF/CMC dispersions are homogeneous thixotropic fluids, we will carry out a detailed investigation of their flow behavior. We will work in the regime where the concentration of MFs is high enough to form a percolating, arrested network, and high enough levels of CMC to prevent significant aggregation and sedimentation. Such conditions can be found at BCMF/CMC weight ratios lower than $\sim 5$ at MF weight fractions higher than $0.1 \mathrm{wt} \%$ (Veen et al., 2014). Viscosity bifurcation measurements will be used to establish the critical stress where MF network breakage (shear rejuvenation) is balanced by reformation. Next we will use rheo-MRI velocimetry to study flow of BCMF/CMC dispersions close to the critical stress (Coussot, Nguyen, Huynh, \& Bonn, 2002; Møller, Mewis, \& Bonn, 2006) and compare with the behavior of typical thixotropic yield stress fluids (Møller, Rodts, Michels, \& Bonn, 2008).

\subsection{Experimental procedures}

\subsubsection{Sample preparation}

Cubes of BC in syrup from eight cups with $220 \mathrm{~mL}$ of lychee/vanilla flavored nata de coco (Kara Santan Pertama, Bogor 16964, Indonesia) were filtered and washed under a demi-water tap. After immersion in $1.5 \mathrm{~L}$ of nanopure water (Barnstead Nanopure Diamond, conductivity $18 \mu \mathrm{S} / \mathrm{cm}$ ) the cubes were cut by a hand blender (Braun 4185545) and washed. Each washing step consisted of rinsing the cellulose by filtration over a vacuum filter (Whatman Schleicher and Schuell 113, wet strengthened circles, $185 \mathrm{~mm}$ diameter) and redispersing the residue in $1.5 \mathrm{~L}$ of nanopure 
water with the hand blender. After eight washing steps the cellulose residue was redispersed in 400 $\mathrm{mL}$ of nanopure water. Several samples containing $0.8 \mathrm{wt} \% \mathrm{BCMF}$ were prepared by dilution with nanopure water and adding different amounts of a concentrated CMC solution (Ashland Blanose Aqualon $99.5 \%$ pure, 9M31XF, $M_{\mathrm{w}} \approx 250 \mathrm{~kg} / \mathrm{mol}$, degree of polymerization 1100 , degree of substitution $0.80-0.95$, density $0.75 \mathrm{~kg} / \mathrm{L}$ ). These samples were passed through a Microfluidizer once (M110S, Microfluidics) with a $z$-chamber of $87 \mu \mathrm{m}$ at a pressure of $120 \mathrm{MPa}$. The weight fraction of BCMF in the resulting mixtures was determined gravimetrically by drying of the dispersions under reduced pressure and elevated temperatures. The average over three samples per $\mathrm{CMC} / \mathrm{BCMF}$ ratio was taken. Dilutions were made by addition of nanopure water and redispersion by gentle shaking.

\subsubsection{Rheology}

Apparatus. Experiments were performed on an Anton Paar Physica MCR301 rheometer equipped with (1) a CP geometry, cone angle $2.0^{\circ}$, cone diameter $4 \mathrm{~cm}$, truncation gap $60 \mu \mathrm{m}$ and (2) a serrated CC (PEEK) geometry, $r_{\mathrm{i}} / r_{\mathrm{o}} 8.5 / 9.5 \mathrm{~mm}$ (gap width $1.0 \mathrm{~mm}$ ).

Flow curves (strain controlled). Flow curves of BCMF/CMC 0.80/0.15, 0.80/0.20 and 0.20/0.05 $\mathrm{wt} \%$ were measured under strain controlled conditions. In CP geometry, we performed a test with an up and down sweep in shear rate. The shear rate was first increased from 0.1 to $500 \mathrm{~s}^{-1}$ in $2 \mathrm{~min}$ and then decreased from 500 to $0.1 \mathrm{~s}^{-1}$ in $2 \mathrm{~min}$. In CC geometry, a flow curve of BCMF/CMC $0.20 / 0.05 \mathrm{wt} \%$ was measured. The shear rate was decreased from $2 \times 10^{2}$ to $2 \times 10^{-3} \mathrm{~s}^{-1}$ over the course of $1 \mathrm{~h}$, after pre-shearing the sample at $200 \mathrm{~s}^{-1}$ for 3 minutes.

Viscosity bifurcation (stress controlled) measurements. For these experiments we used CC geometry and BCMF/CMC 0.20/0.05 wt\% dispersions. In each experiment a fresh sample was used that was pre-sheared at $200 \mathrm{~s}^{-1}$ for 3 minutes after which a fixed stress was applied. The rate of strain was then measured as a function of time.

\subsubsection{Rheo-MRI velocimetry}

Time-averaged velocity profiles were measured according to procedures by Callaghan et al. (Callaghan, 1993; 1999) on a Bruker Avance II spectrometer at 7.0 T magnetic field strength (resonance frequency $300 \mathrm{MHz}$ for ${ }^{1} \mathrm{H}$ ). The magnet was equipped with a Bruker rheo-MRI accessory in combination with two measurement geometries: (1) a $\mathrm{CP}$, cone angle $7.0^{\circ}$, cone diameter $1 \mathrm{~cm}$ (PEEK) and (2) a serrated CC, $r_{\mathrm{i}} / r_{\mathrm{o}} 8.5 / 9.5 \mathrm{~mm}$ (PEEK).

\subsection{Results and Discussion}

Addition of small amounts of CMC to a BCMF dispersions not only leads to a more homogeneous distribution of MFs at the sub-micron scale (SI, section S7.1a), but also to significant improved stability against aggregation. For plain dispersions of BCMF, mild shear induces a "log-rolling" effect, which is absent when CMC is added. This can be visualized by 
imaging the local density of BCMC using MRI $R_{2}$ contrast when dispersions with and without CMC are sheared in a CC geometry, as is shown in Figure 7.1. The BCMF/CMC dispersion shows no sign of such shear-induced heterogeneities and appears homogeneous at the rheometer (macro)scale.
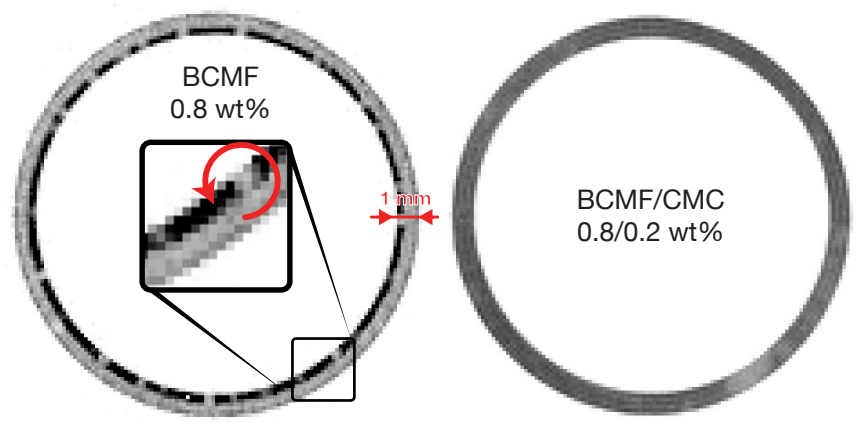

Figure 7.1: $R_{2}$-contrast MRI images of the annulus of a $r_{\mathrm{i}} / r_{\mathrm{o}} 8.5 / 9.5 \mathrm{~mm}$ CC geometry after mild preshear of $5 / \mathrm{s}$ during 3 minutes (slice thickness $1.0 \mathrm{~cm}$ ). The presence of cellulose MFs leads to faster relaxation of the NMR signal and hence to a larger $R_{2}$-value (i.e., brighter areas contain more MFs). Under these circumstances, no strong contrast can be observed in the BCMF/CMC dispersion, while in the BCMF dispersion large flocs (logs) have formed that span part of the gap.

In strain-controlled flow curves of BCMF/CMC dispersions (Figure 7.2), we can see that the dispersions are strongly shear thinning, possibly due to orientation of the MFs in the flow direction (Iotti et al., 2010), and that the concentrations of MFs and CMC are (unsurprisingly) reflected in the bulk viscosity.

Higher MF concentrations lead to an upward shift in viscosity of the whole flow curve, whereas the viscosity effect of CMC can only be seen at higher shear rates. Local measurements of the apparent viscosity of the continuous phase from the mobility of nanoparticles by diffusion NMR (Bourouina et al., 2015; de Kort, Rombouts, Hoeben, Janssen, Van As, et al., 2015a; de Kort, van Duynhoven, Hoeben, Janssen, \& Van As, 2014; de Kort, van Duynhoven, Van As, \& Mariette, $2015 b$ ), and an assessment of the molecular mobility of CMC by ${ }^{1} \mathrm{H}$ NMR spectroscopy show that at most $5 \%$ of CMC closely associates with the MFs, while the rest remains dissolved in solution (SI, section S7.1b). In the high shear rate regime, the dispersive effect of the addition of CMC leads to an increase of bulk viscosity significantly larger than only the contribution of the viscosity of the continuous phase (which is $<6 \mathrm{mPa}$ ). 


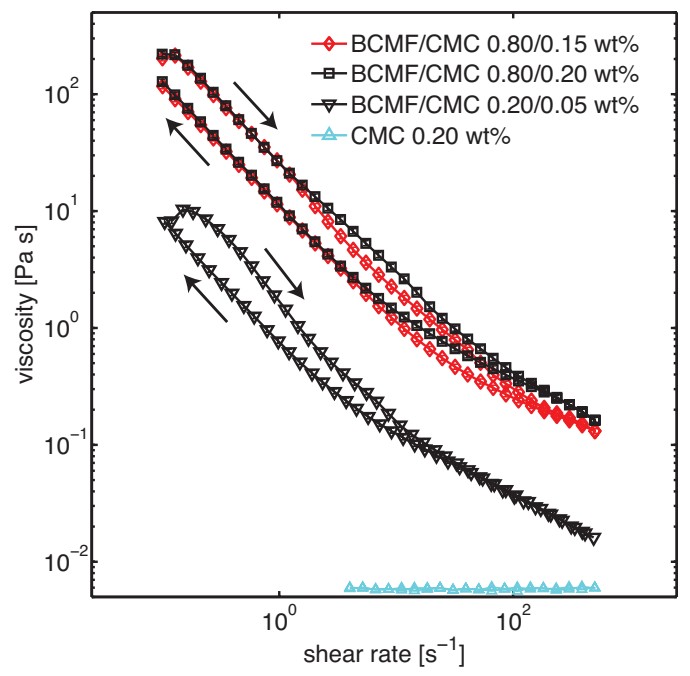

Figure 7.2: Strain-controlled macroscopic flow curves of dispersions of different BCMF concentrations and $\mathrm{BCMF} / \mathrm{CMC}$ ratios. The shear rate was first increased from 0.1 to $500 \mathrm{~s}^{-1}$ in 2 min and then decreased from 500 to $0.1 \mathrm{~s}^{-1}$ in $2 \mathrm{~min}$ (see arrows). The viscosity of CMC $0.2 \mathrm{wt} \%$ could only be determined in part of this domain due to limited sensitivity of the rheometer.

The down-sweep flow curve shows lower viscosities than the up-sweep, and this behavior repeats for every successive sweep. In other work, the time-dependent rheology and thixotropy of cellulose MF dispersions has been demonstrated (Iotti et al., 2010; Lowys, Desbrieres, \& Rinaudo, 2001). The viscosity decrease seen in Figure 7.2 is therefore likely caused by the thixotropy of the dispersions, implying that flow curves measured by a shear rate sweep (such as in Figure 7.2) do not necessarily reflect steady-state properties. Assuming the MF dispersions are indeed thixotropic, we need to monitor the development of the viscosity under a single imposed stress as a function of time in order to measure the steady-state flow properties of the dispersions.

Viscosity bifurcation behavior. Thixotropy and yield-stress are both associated with the continuous breakage and reformation of the particle (fiber) network and are therefore thought to be intrinsically linked (Coussot et al., 2002; Møller et al., 2006). In steady state, under a constant stress, a kinetic balance between network breakage (shear rejuvenation) and reformation (aging) determines whether or not a thixotropic yield stress fluid can flow. There exists a critical stress $\sigma_{\mathfrak{c}}$, above which shear rejuvenation outcompetes aging so that the fluid can flow homogeneously, and below which aging outcompetes shear rejuvenation, eventually resulting in an infinite viscosity (i.e., in a solid) and no flow, even if the material was flowing initially. This phenomenon is known as the viscosity bifurcation (Coussot et al., 2002), and implies that in steady state, under 
controlled stress, a wide range of shear rates is inaccessible, namely those shear rates below the critical shear rate $\dot{\gamma}_{c}$, which is the shear rate at the critical stress $\dot{\gamma}\left(\sigma_{c}\right)$.

To demonstrate this behavior for cellulose microfibril dispersions, we performed viscosity bifurcation experiments for a selected BCMF/CMC dispersion (0.20/0.05 wt\%) in CC geometry. In these experiments, we monitored the shear rate as a function of time under a fixed stress. Immediately prior to each experiment, we erased the shear history of the sample by a shearing at $200 \mathrm{~s}^{-1}$ for three minutes. We compare the resulting steady-state shear rates with flow curves acquired through a slow strain sweep. The results are presented in Figure 7.3.
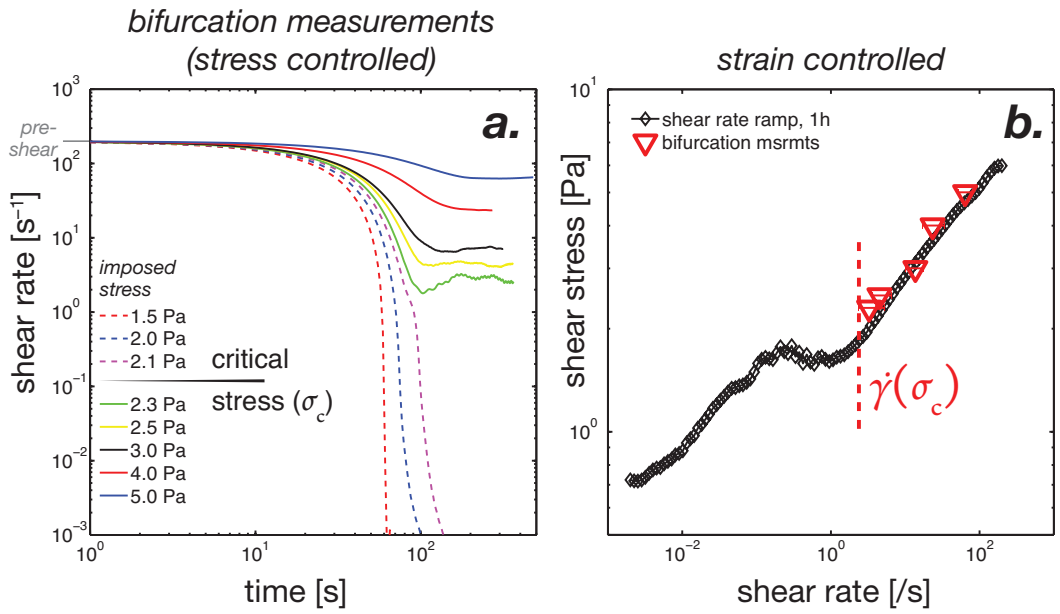

Figure 7.3: (a) Viscosity bifurcation measurements on BCMF/CMC 0.20/0.05 wt $\%$ in CC geometry. Before imposing constant stress, the dispersions were pre-sheared at $200 \mathrm{~s}^{-1}$ for three minutes. (b) Results of the viscosity bifurcation experiments superimposed onto a slowly acquired $(1 \mathrm{~h})$ strainsweep flow curve. The apparent critical shear rate $\dot{\gamma}_{\mathrm{c}}$ is indicated with a dotted line.

The existence of a critical stress is evident from the viscosity bifurcation experiments (Figure 7.3a), which show that below a critical stress the initial flow of the fluid comes to a halt (dotted lines). For stresses higher than the critical stress, the shear rate develops towards a steady state value (solid lines). We can overlay these steady-state shear rates onto a flow curve that was obtained by slow a strain sweep, so that it should be close to steady state behavior. The slowly acquired strain-sweep flow curve of Figure 7.3b is very similar to flow curves of cellulose MF dispersions previously presented in the literature (e.g., ref (Martoïa et al., 2015)). We find that the steady-state shear rates from the stress-controlled experiments overlap with this strain-controlled flow curve. However, a wide range of shear rates below the apparent critical shear rate $\dot{\gamma}\left(\sigma_{c}\right)$ is inaccessible under stress-controlled conditions. Moreover, one would expect strain-controlled flow curves to be flat below the critical shear rate, as demonstrated for instance for dispersions of 
mildly attractive silica particles by Møller et al. (Møller et al., 2008) The unusual features that can be seen in the flow curves (such as the negative slope at shear rates around $10 \mathrm{~s}^{-1}$ ) cannot be accounted for by the viscosity bifurcation model or the more conventional Herschel-Bulkley model, which describe typical flow curves of yield-stress fluids. Effects similar to those seen in Figure $3 \mathrm{~b}$ have been described by Ovarlez et al. as typically occurring below the critical stress in some soft-jammed materials (Ovarlez, Rodts, Chateau, \& Coussot, 2009). In such cases, the observation of a stress plateau within limited range of shear rates is typically caused by shear localization within a thin layer in the material. Our cellulosic material is not a jammed system, for which reason we should be careful to apply such conclusions directly to this material. We therefore measured flow profiles (velocity as a function of position in the gap) in order to check whether the flow is indeed localized in only a narrow part of the sample.

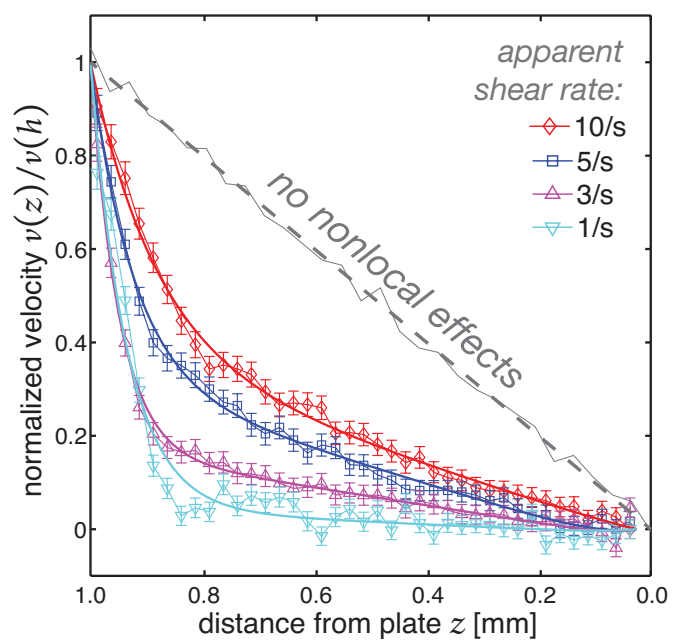

Figure 7.4: Flow profiles (velocity normalized to the cone velocity) of BCMF/CMC 0.20/0.05 wt\% in CP geometry. Solid lines are fits to the nonlocal model (Goyon, Colin, Ovarlez, Ajdari, \& Bocquet, 2008). The gap is $1.0 \mathrm{~mm}$ wide at the position where the profile is measured. The CP geometry is not sandblasted, which leads to slippage at the plate (i.e., the velocity drops to zero over a very small distance, cf. SI, section S7.2). By extrapolation of the last five pixels at the plate (as in ref (Goyon et al., 2008)), we could correct the flow profile for the slippage. The dashed line represents the flow profile of non-thixotropic yield stress fluids such as dense suspensions of soft particles in a homogeneous stress field with a gap much larger than the particle size (Ovarlez, Cohen-Addad, Krishan, \& Goyon, 2013). Indeed, the flow profile of a hair gel containing carbopol (in gray) is linear, implying the absence of non-locality. 
Nonlocal effects. Thixotropic yield stress fluids flowing profiles in homogeneous stress fields typically have a perfectly linear flow profile at imposed shear rates $>\dot{\gamma}_{c}$, i.e., there is a single shear rate across the gap. In case a shear rate $\left\langle\dot{\gamma}_{\mathrm{c}}\right.$ is imposed upon the material, it accommodates this deformation through shear banding: One shear band flows with shear rate $\dot{\gamma}_{c}$; This band can not span the whole gap, so that part of the material does not move $(v=0)$ (Møller et al., 2008).

We measured such (time-averaged) flow profiles by rheo-MRI velocimetry (Figure 7.4). A $7^{\circ} \mathrm{CP}$ geometry was used, because here the spatial variation of the stress across the gap $\sigma(z)$ is only $1.5 \%$ (Cheng, 1966) and can be safely ignored (Møller et al., 2008), unlike for flow in CC geometry or in pipes (T. Q. Li et al., 1994). The flow profiles were measured in the shear rate regime close to the critical shear rate $\left(\sim 1-10 \mathrm{~s}^{-1}\right)$. Since the local viscosity $\eta(z)=\frac{\sigma_{0}}{\dot{\gamma}(z)}$, where $\sigma_{0}$ is the (nearly) $z$-independent stress, and the local shear rate in CP geometry $\dot{\gamma}(z)=\frac{\partial v(z)}{\partial z}$, the strong non-linearity of the flow profiles in Figure 7.4 directly reflects local viscosity differences across the gap. The flow profiles thus differ strongly from the typical picture, where for shear rates (a) higher than $\dot{\gamma}_{c}$ the flow profile is linear or (b) lower than $\dot{\gamma}_{c}$ the flow profile is banded into a region without flow and a region with shear rate $\dot{\gamma}_{c}$ (Møller et al., 2008). In our case, there is flow throughout the gap, while the local shear rate is not constant. This implies that the viscosity varies spatially across the gap.

A recent model by Goyon et al. (Goyon et al., 2008) can account for such spatial variation of the viscosity. The model introduces a length scale $\xi$ at which nonlocal effects can lead to spatial variations in the viscosity even if the stress is homogeneous (Goyon, Colin, \& Bocquet, 2010). Fluidity is the reciprocal of viscosity, and its spatial variation can be described as $f(z)=f_{\text {bulk }}+\xi^{2} \frac{\partial^{2} f(z)}{\partial z^{2}}$, where $f_{\text {bulk }}$ is the fluidity of the bulk and $\xi$ a flow cooperativity length. Different explanations have been given as to the physical meaning of the cooperativity length, depending on the type of material studied, suggesting that it is the distance over which plastic events influence each other (Kamrin \& Koval, 2012), but that it is not necessarily a material property (de Cagny, Fall, Denn, \& Bonn, 2015). Ignoring the minor spatial variation of the stress in our CP geometry, we can integrate over $z$ to obtain an expression for the velocity across the gap (no-slip boundary conditions, cf. SI, section S7.3):

$v(z)=\sigma_{0} f_{\text {bulk }} z+\sigma_{0}\left(f_{z=0}-f_{\text {bulk }}\right) \xi \sinh \left(\frac{z}{\xi}\right)+\frac{v(h)-\sigma_{0} f_{\text {bulk }} h-\sigma_{0}\left(f_{z=0}-f_{\text {bulk }}\right) \xi \sinh \left(\frac{h}{\xi}\right)}{\cosh \left(\frac{h}{\xi}\right)-1}\left[\cosh \left(\frac{z}{\xi}\right)-1\right]$,

where $h$ is the local height of the gap, $v(h)$ the velocity of the cone, $f_{z=0}$ the fluidity at the plate, and $\sigma_{0}$ the homogeneous stress. The model accounts for the observed spatial variation of the viscosity with a flow cooperativity length $\xi$ of $0.08 \pm 0.03 \mathrm{~mm}$. Nonlocal effects have been observed in cases where the flow of a fluid is strongly confined, i.e., the width of the channel or gap in which the fluid flows is only one or two decades larger than the size of the dispersed particles. In BCMF gels in absence of CMC, at $0.20 \mathrm{wt} \% \mathrm{MF}$ concentration, there is a broad, lognormal floc size distribution 
in the 1-100 $\mu \mathrm{m}$ range with a medium floc size of $15 \mu \mathrm{m}$ (Kuijk et al., 2013). The length scale of these heterogeneities is comparable to $\xi$, implying that they might persist in BCMF dispersions even after addition of CMC. This could effectively render the dispersions confined within the CP geometry, as the gap at the measurement position is $1.0 \mathrm{~mm}$ wide. Although the presence of CMC makes the dispersions clearly more homogeneous the submicron scale and mitigates progressive macroscale aggregation of the microfibrils (Figure 7.1), it apparently does not lead to completely homogeneous dispersions at the micron scale. Another explanation for the observed non-locality could be migration or dilatation of such micron-size particles under shear, leading to density differences and consequently viscosity differences across the gap. Such effects have been observed for granular materials and suspensions with element sizes on the order of the gap width (de Cagny et al., 2015; Nichol, Zanin, Bastien, Wandersman, \& van Hecke, 2010). This explanation would still be consistent with the homogeneous density of the BCMF/CMC dispersion as shown in Figure 7.1 (measured in the absence of shear), if the density differences that arise under shear quickly disappear after flow has stopped.

The shear rates imposed when measuring the flow profiles presented in Figure 7.4 were similar to the critical shear rate $\dot{\gamma}_{\mathrm{c}}$ found by the viscosity bifurcation experiments. This means that, due to the strong curvature of the flow profiles, the local shear rate, which is given by the slope of the flow velocity profiles particularly at the lower imposed shear rates, drops significantly below $\dot{\gamma}_{\mathrm{c}}$. This is not consistent with the picture that the local shear rate cannot be lower than $\dot{\gamma}_{\mathrm{c}}$. The sharp transitions between sheared and stationary regions as observed for typical thixotropic yield-stress fluids in CP geometry, are apparently broadened in BCMF dispersions, as is also the case for granular media (Bouzid, Trulsson, Claudin, Clément, \& Andreotti, 2013; Nichol et al., 2010). This might also underlie the continuation of the strain-controlled flow curve of Figure $7.3 \mathrm{~b}$ below the apparent critical shear rate. To distinguish between those cases would require a study of a thixotropic colloid with a well-defined particle size on the order of the gap width in a homogeneous stress field at shear rates close to the critical shear rate.

As recently demonstrated by Paredes et al. (Paredes, Shahidzadeh, \& Bonn, 2015), nonlocal effects due to the presence of large particles should be distinguished from wall slip due to the nonaffinity of particles with the wall, for which can be corrected before the nonlocal model is applied (Goyon et al., 2008). True wall slip of cellulose MF dispersions is notoriously difficult to be prevented in typical rheometer setups, because of the weak affinity of the cellulose fibers with most surfaces, and possibly depletion effects. Both wall slip and nonlocal effects, however, contribute to the macroscopic observation of wall slip. Such apparent wall slip can be observed by varying the gap width in PP geometry, and is indeed significant in the case of BCMF dispersions (cf. SI, section S7.4). The relative contributions of true wall slip and nonlocal effects to the apparent macroscopic wall slip will vary as a function of applied stress and cannot be predicted. 


\subsection{Conclusions}

Although CMC adsorbs only partly to cellulose MFs, addition of CMC leads to a significantly more homogeneous colloid at the sub-micron and macroscopic scales. For this reason, we expected the flow behavior of $\mathrm{BCMF} / \mathrm{CMC}$ dispersions to resemble that of microscopically homogeneous thixotropic fluids. We first showed that BCMF/CMC dispersions have a critical stress and shear rate below which there cannot be steady flow under stress-controlled conditions, which is a typical flow characteristic of thixotropic fluids. On the other hand, strain-controlled flow curves deviated from the typical picture for thixotropic fluids. To see why these differences arose, we measured flow profiles of the BCMF/CMC dispersions by rheo-MRI velocimetry. In CP geometry, where the stress variation across the gap is small (1.5\%), the flow profiles were neither linear and nor showed shear banding that is expected at shear rates around the critical shear rate. Instead, there was flow throughout the gap with a highly curved velocity profile. Given the homogeneous stress distribution in the gap, the non-linearity of the profiles directly reflected differences in viscosity across the dispersions. We used a nonlocal model to describe the viscosity differences across the gap, from which we extracted a flow cooperativity length $\xi$ of $0.08 \pm 0.03 \mathrm{~mm}$ was found, comparable to floc sizes in BCMF gels without CMC. The origin of the nonlocal effects lies in the persistence of micronscale heterogeneities that are of the order of the gap width, despite the apparent homogeneity of the dispersions at the sub-micron and macro scales. These heterogeneities have to be considered when the bulk rheology of BCMF/CMC dispersions is studied in relatively small geometries. The results of this study might also pertain to nonmicrofibrillated cellulosic materials in the presence of charged polymer, including foods such as ketchup and applesauce that naturally include the charged polymer pectin (Derakhshandeh, Kerekes, Hatzikiriakos, \& Bennington, 2011).

\section{Supporting Information}

Supporting information is included below: S7.1a. Microstructural characterization of BCMF/CMC dispersions. S7.1b. Quantification of the amount of mobile CMC in BCMF/CMC dispersions, S7.2. Uncorrected flow profiles in cone-plate geometry, S7.3. Solution of the nonlocal model for Couette flow (homogeneous stress), S7.4. Apparent wall slip.

\section{Acknowledgments}

The authors thank Yuval Mulla, Wolf Rombouts, Jinfeng Peng, Paul Venema, Tatiana Nikolaeva (Wageningen University), Caroline Remijn, Gert-Jan Goudappel, Peter Versluis, and Donny Merkx (Unilever R\&D) for their contributions. This research received funding from NanoNextNL and the Netherlands Organization for Scientific Research (NWO) in the framework of the Technology Area COAST. 


\section{S7.1 a Microstructural characterization of BCMF/CMC dispersions}

In the presence of CMC, when added in the process of deagglomeration, the distribution of microfibrils over the available volume is more homogeneous, but heterogeneity persists on larger scales due to incomplete deflocculation (Veen et al., 2015). CMC is thought to associate with cellulose mainly through hydroxyl-mediated hydrogen bonding. For example, one early study showed that $\mathrm{CMC}$ can interfere with the in vivo assembly of $\mathrm{BC}$ microfibrils (i.e., linear aggregates of cellulose chains) (Yamamoto \& Horn, 1994), where the degree of substitution (DS) of hydroxyl groups of the CMC chain by carboxymethyl groups plays a critical role (Haigler et al., 1982). CMC that is not fully substituted with carboxymethyl groups has hydroxyl groups remaining that can, in principle, take part in hydrogen bonding. It is thought that $\mathrm{BC}$ microfibril formation is impacted by these hydrogen-bonding interactions.

We investigate the associative behavior of $\mathrm{CMC}$ in the dispersions by performing local viscosity measurements for which we use ${ }^{19} \mathrm{~F}$-labeled nanoparticles. Their mobility is observed by ${ }^{19} \mathrm{~F} \mathrm{NMR}$ diffusometry and locally probes the viscosity of the continuous phase.

\section{S7.1a.1 Experimental procedures}

AFM. A $5 \mu \mathrm{L}$ aliquot of microfibril dispersion was deposited on a silica wafer. The silica wafer was glow-discharged under vacuum before being used to improve the surface hydrophilicity. Afterward, the samples were dried in air, washed with deionized water, and dried again under a nitrogen flow to remove nonadsorbed material. The sample was analyzed with a Bruker Nanoscope V instrument in peak force tapping mode. Here, the AFM tip oscillates in a sinusoidal manner in the vertical direction, while the peak force is used as a feedback signal. This mode allows for direct force control and avoids damage due to lateral forces. Image contrast is provided by the peak force error (the feedback signal). Peak force images offer better resolution than height images, especially for soft samples (Alsteens et al., 2012). Silicon nitride tips with a typical radius of $2 \mathrm{~nm}$ (Bruker SCANASYST-AIR) were used for samples in the dilute regime, and silicon nitride tips with a typical radius of $20 \mathrm{~nm}$ (Bruker NP-10) were used for samples in the concentrated regime.

Cryo-SEM. A droplet of sample was placed on top of a rivet and plunge-frozen in melting ethane. The sample was cryo-planed using a cryo-ultramicrotome (Leica Ultracut UCT EM UC7/FC7), to obtain a freshly prepared cross-section. Cryo-planing was done first with a section thickness of $100 \mathrm{~nm}$ at a speed of $50 \mathrm{~mm} / \mathrm{s}$ using a glass knife. The last sections were made at decreasing thickness, down to $20 \mathrm{~nm}$, with a speed of $2 \mathrm{~mm} / \mathrm{s}$ using a diamond knife (Diatome histo cryo 8 $\mathrm{mm})$ at $-120^{\circ} \mathrm{C}$. The rivet was mounted onto a holder and transferred into a Gatan Alto 2500 preparation chamber. To reveal microstructures under the planed surface, the temperature of the sample was increased for a short while to $-90^{\circ} \mathrm{C}$ to remove a thin layer of water by sublimation. This yielded a $3 \mathrm{D}$ view of the planed sample. The sample was sputter-coated with platinum $(120 \mathrm{~s}$, 
$10 \mathrm{~mA}$ ) for a better SEM contrast and to prevent charging by the electron beam. The sample was imaged using a Zeiss Auriga field-emission SEM at $-125^{\circ} \mathrm{C}$ and an accelerating voltage of $3 \mathrm{kV}$.

Nanoparticle diffusometry. ${ }^{19} \mathrm{~F}$ diffusion-ordered spectroscopy (DOSY) was performed on a Bruker Avance II spectrometer at 7.0 T magnetic field strength (resonance frequency $282 \mathrm{MHz}$ for ${ }^{19} \mathrm{~F}$ ), equipped with a Bruker diff25 gradient probe (maximum pulsed field gradient intensity 9.60 $\mathrm{T} / \mathrm{m}$ ). The probe was equipped with a $10-\mathrm{mm}$ rf insert tuned to the ${ }^{19} \mathrm{~F}$ resonance frequency. The samples contained $0.1 \mathrm{wt} \%{ }^{19} \mathrm{~F}$ labeled, nonsticky dendritic nanoparticles with a hydrodynamic radius of $7.5 \mathrm{~nm}$ (de Kort et al., 2014; de Kort, Rombouts, Hoeben, Janssen, Van As, et al., 2015a). The overall sample volume was chosen as to not exceed the linear part of the magnetic field gradient. Sample temperature was kept at 293 K. DOSY experiments were performed by stepwise variation of the gradient pulse amplitude, while keeping the diffusion-observation time and gradient pulse duration constant. The attenuation of the NMR echo intensity as a function of increasing gradient amplitude is described by the Stejskal-Tanner equation $A=A_{0} \mathrm{e}^{-b D}$, where $A$ is the echo intensity, $D$ the self-diffusion coefficient $\left(\mathrm{m}^{2} / \mathrm{s}\right)$ and $b=(\gamma \delta g)^{2}(\Delta-\delta / 3)$, where $\gamma$ is the gyromagnetic ratio of the observed nucleus $\left(25.18 \times 10^{7} \mathrm{rad} /(\mathrm{T} \mathrm{s})\right.$ for $\left.{ }^{19} \mathrm{~F}\right), g$ the magnetic field gradient amplitude $(\mathrm{T} / \mathrm{m}), \Delta$ the effective observation time $(\mathrm{s})$ and $\delta$ the effective gradient pulse duration (s), where $\delta \ll \Delta$ (narrow gradient pulse approximation) (Stejskal \& Tanner, 1965). We used stimulated echo-based DOSY experiments with unipolar, ramped gradient pulses. In all experiments, an effective observation time $\Delta$ of $100 \mathrm{~ms}$ was used, and an effective gradient pulse duration $\delta$ of $5 \mathrm{~ms}$. Gradient amplitude $g$ was varied logarithmically between $0.10-9.60 \mathrm{~T} / \mathrm{m}$ in 128 steps. The NMR signal was averaged between 16-64 times at each gradient amplitude, with a repetition time of $1 \mathrm{~s}$. DOSY spectra were obtained through Fourier transformation of the stimulated echo for each gradient step and subsequent phasing using standard procedures. The signals in each of the resulting ${ }^{19} \mathrm{~F}$ NMR spectra were integrated to obtain the attenuation curve.

\section{S7.1a.2 Results and Discussion}

Impact of CMC addition to BCMF on macroscopic and local viscosity. In Figure S7.1A, a cryo-SEM micrograph of microfibrils in the absence of CMC is presented, where the distribution of microfibrils is strongly heterogeneous. In Figure S7.1B, it can be seen that addition of CMC (not visible) leads to a more homogeneous distribution of $\mathrm{BC}$ microfibrils over the available volume at the micron scale. 

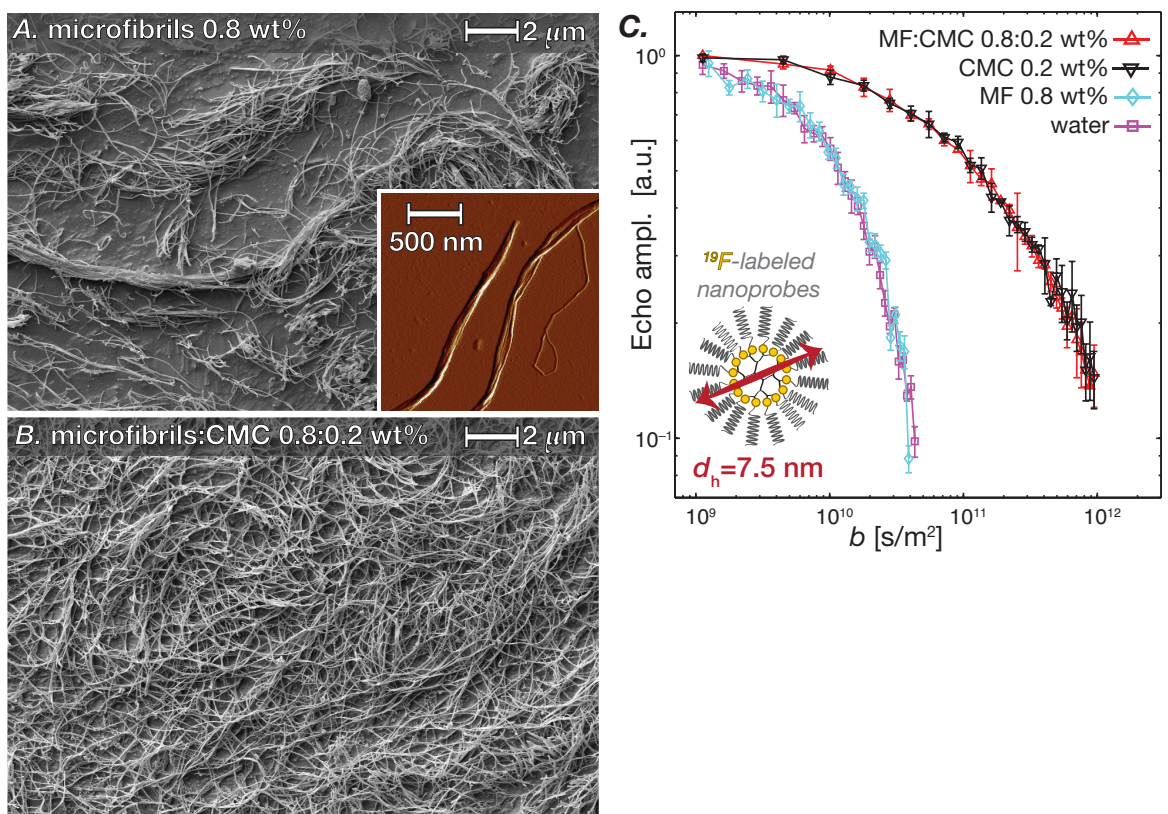

Figure S7.1: (A) Cryo-SEM micrograph of a $0.8 \mathrm{wt} \%$ microfibrils (MF) dispersion. Inset: AFM image of a single microfibril (additional AFM images included in Figure S7.2) (B) Cryo-SEM micrograph of a BCMF/CMC 0.8/0.2 wt\% dispersion. (C) PFG ${ }^{19} \mathrm{~F}-\mathrm{NMR}$ response curves of nanoparticles in water, MF $0.8 \mathrm{wt} \%$, CMC $0.2 \mathrm{wt} \%$ and BCMF/CMC 0.8/0.2 wt\%. These nanoparticles are ${ }^{19} \mathrm{~F}$-labeled PEGylated dendrimers with a diameter of $7.5 \mathrm{~nm}$, as shown in the cartoon.
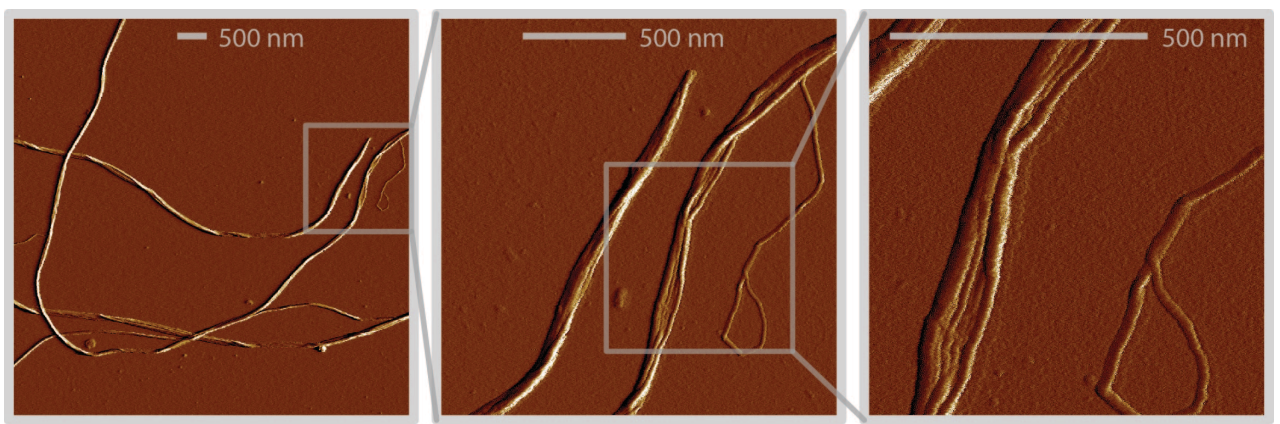

Figure S7.2: AFM micrographs of cellulose microfibrils (peak force error contrast). Individual fibers have a high aspect ratio (leftmost image). They are ribbon shaped, and can be seen twisting (middle image). The ribbons consist of laterally stacked elementary fibrils, which can individually protrude from the microfibrils (rightmost image). 
As CMC is not visible in these images, we cannot see its spatial distribution and the manner in which it associates with the microfibrils. Therefore, we probe the apparent viscosity of the continuous phase in situ by measuring the mobility of nonsticky nanoparticles (Bourouina et al., 2015; de Kort, van Duynhoven, Van As, \& Mariette, 2015b). These nanoparticles are much smaller than the mesh size of the MF network and their mobility is therefore expected not to be influenced by the MF network. In Figure S7.1C, we can see PFG ${ }^{19} \mathrm{~F}-\mathrm{NMR}$ response curves of ${ }^{19} \mathrm{~F}$ labeled, PEGylated dendrimers with a hydrodynamic diameter of $7.5 \mathrm{~nm}$. (de Kort et al., 2014; de Kort, Rombouts, Hoeben, Janssen, Van As, et al., 2015a) The decay of echo amplitude $A$ is plotted as function of $b$, which is proportional to the square of the pulsed magnetic field gradient intensity. The curves decay according to the Stejskal-Tanner equation $A=A_{0} e^{-b D}$, where $D$ is the particle self-diffusion coefficient (Stejskal \& Tanner, 1965). The echo amplitude decay curves of the nanoparticles in water (bulk viscosity $\eta=1.0 \mathrm{mPa}$ s) and in $0.8 \mathrm{wt} \%$ MFs overlap, demonstrating that the particles indeed probe the apparent viscosity of the continuous phase, which is not significantly different between both systems. It turns out that also the response curves of $0.2 \mathrm{wt} \%$ CMC $(\eta=5.8 \pm 0.3 \mathrm{mPas})$ and $0.8 / 0.2 \mathrm{wt} \% \mathrm{BCMF} / \mathrm{CMC}$ overlap. We note that the CMC solution was microfluidized just like the BCMF/CMC dispersion to allow for unbiased comparison.

\section{S7.1b Quantification of the amount of mobile CMC in BCMF/CMC dispersions}

This prompted us to quantify the amount of soluble CMC in situ in the dispersion, as well as in the supernatant upon centrifugation. The level of unbound CMC in BCMF/CMC dispersions was determined by two independent ${ }^{1} \mathrm{H}$ NMR methods. In one method the level of unbound CMC was determined in situ (Figure S7.3), and in the concentration range accessible to this method ( $>0.05 \mathrm{wt} \%)$ no significant difference between the amount of CMC put into the dispersion, and the amount of mobile CMC could be observed. Binding of CMC leads to decrease in mobility and hence disappearance of the NMR signal.

The level of mobile CMC in the dispersions was also determined by measuring the degree of recovery of CMC in the supernatant of centrifuged BCMF/CMC dispersions (Figure S7.4). This method can access lower CMC levels (0.01-0.07 wt\%) and shows near-complete recovery of CMC in the supernatants of BCMF/CMC dispersions. We verified that no BCMF had remained in the supernatant by comparing the ${ }^{1} \mathrm{H}$ NMR spectra of the Seaman hydrolysates (Figure S7.5). The ratio of glucose to carboxyl-glucose was similar in a pristine CMC solution and the supernatant of a BCMF/CMC dispersion.

The results of both the diffusometric and spectroscopic approaches show that within experimental error (5\%) the amount of mobile CMC equals the dosed concentrations of respectively 0.2 and $0.8 \mathrm{wt} \%$. These results consistently indicate that a smaller $(<5 \%)$ fraction of CMC is adsorbed to the BC microfibrils than previously estimated (Veen et al., 2015). 


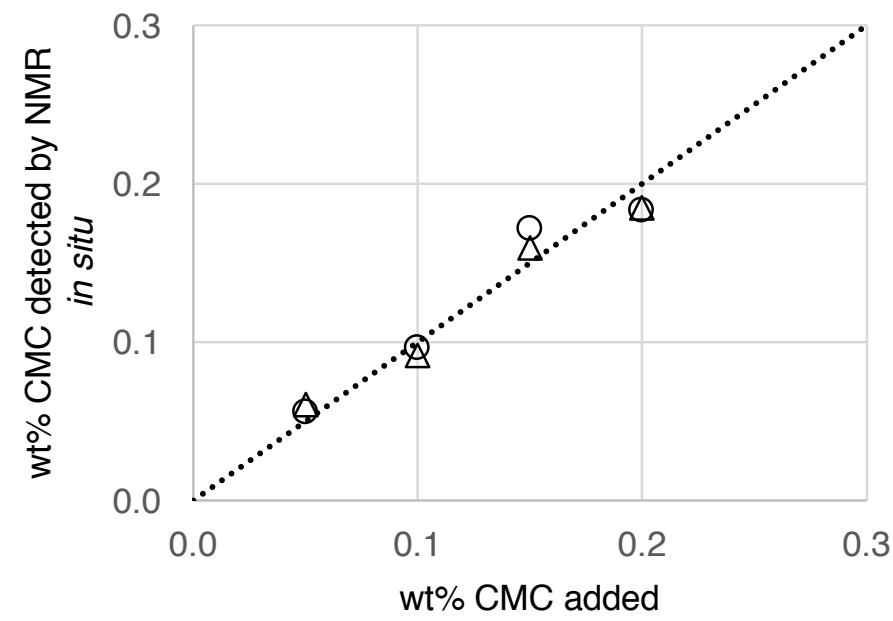

Figure S7.3: The amount of mobile CMC in BCMF/CMC dispersions was measured in situ from the ${ }^{1} \mathrm{H}$ NMR signal intensity of CMC between $2.94-4.28 \mathrm{ppm}$ on a Bruker Avance III spectrometer operating at $600 \mathrm{MHz}$ using the Eretic signal as an internal standard. The lineshape of CMC ${ }^{1} \mathrm{H}$ NMR resonances in the $\mathrm{BCMF} / \mathrm{CMC}$ dispersions was similar to that in pristine $\mathrm{CMC}$ solutions. Circles and triangles represent experimental repetitions. The concentration of mobile $\mathrm{CMC}$ was calculated from a concentration response curve of CMC solutions with known concentrations. 


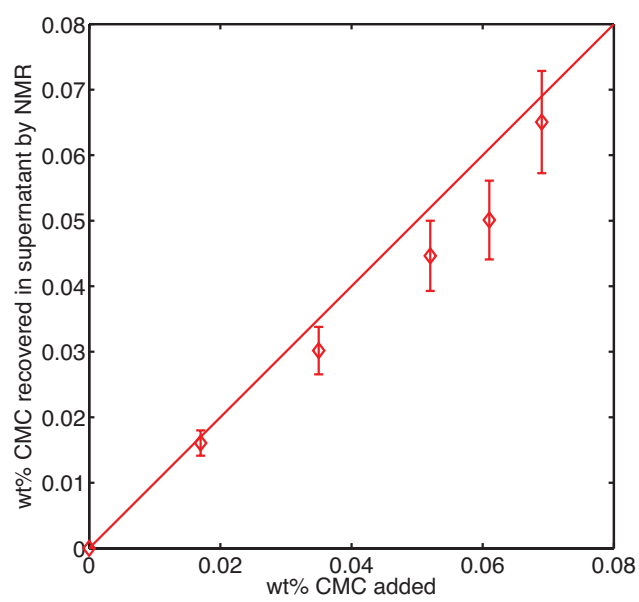

Figure S7.4: Amount of mobile CMC in BCMF/CMC dispersions as determined from CMC recovery of CMC in the supernatant upon centrifugation. The amount of CMC added into the dispersions is plotted against the content in the supernatant. CMC content was determined by quantitative monosaccharide analysis upon Saeman hydrolysis. Samples are measured in $14 \mathrm{wt} \% \mathrm{D}_{2} \mathrm{SO}_{4}$ in $\mathrm{D}_{2} \mathrm{O}$ on a Bruker Avance III spectrometer operating at $600 \mathrm{MHz}$. The glucose anomeric proton peaks $[\delta$ 4.8-3.9 ppm] and carbomethoxyl $\mathrm{CH}_{2}$-peaks [ $\left.\delta 3.58-3.54 \mathrm{ppm}\right]$ are used for the quantification. The glucose:carbomethoxyl molar ratio (4:1) remains constant for all the BCMF/CMC-dispersions, indicating that no additional glucose from $\mathrm{BCMF}$ is introduced in the supernatant. 


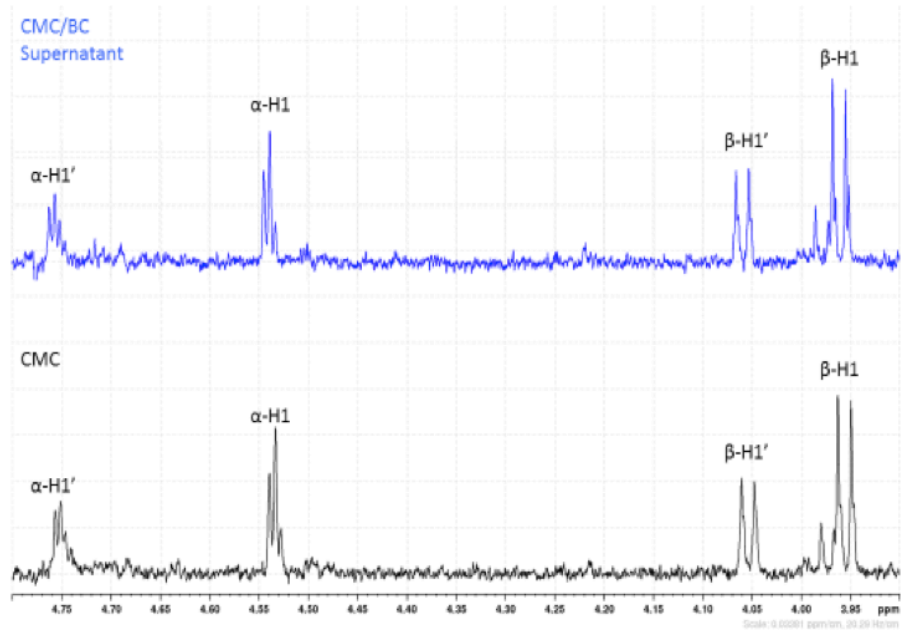

Figure S7.5: Anomeric region of the $600 \mathrm{MHz}{ }^{1} \mathrm{H}-\mathrm{NMR}$ spectrum of two hydrolysed CMC-containing systems in $14 \% \mathrm{D}_{2} \mathrm{SO}_{4}$ in $\mathrm{D}_{2} \mathrm{O}$. In this spectral area, the anomeric protons of glucose $(\alpha-\mathrm{H} 1+\beta-\mathrm{H} 1)$ and carboxymethylated glucose $(\alpha-\mathrm{H} 1+\beta-\mathrm{H} 1)$ are present. The top spectrum (hydrolysed supernatant of $\mathrm{BCMF} / \mathrm{CMC}$ ) is not significantly different from the bottom spectrum (hydrolysed pristine CMC solution), implying that no compound besides $\mathrm{CMC}$ is present in the supernatant after centrifugation of the BCMF/CMC dispersion. 


\section{S7.2. Uncorrected flow profiles in cone-plate geometry}

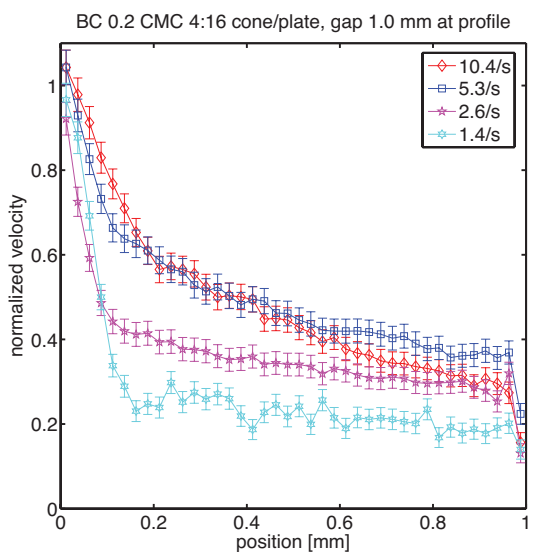

Figure S7.6: Uncorrected flow profiles in cone/plate geometry, where slippage at the (smooth) plate is visible. This was subtracted by extrapolation of the last five pixels at the plate and subtracting the slip velocity. 


\section{S7.3. Solution of the nonlocal model for Couette flow (homogeneous stress)}

The nonlocal model from ref (Goyon et al., 2008):

$f(z)=f_{\text {bulk }}+\xi^{2} \frac{\partial^{2} f(z)}{\partial z^{2}}$, where $f(z)=\frac{1}{\eta(z)}=\frac{\dot{\gamma}(z)}{\sigma(z)}$

For flows between two parallel plates (Couette flow), where the stress throughout the liquid is homogeneous $\left(\sigma(z)=\sigma_{0}\right)$, the solution to this equation is:

$\sigma_{0} f(z)=\sigma_{0} f_{\text {bulk }}+A \cosh \left(\frac{z}{\xi}\right)+B \sinh \left(\frac{z}{\xi}\right)$

Condition 1: $A=\sigma_{0}\left(f_{z=0}-f_{\text {bulk }}\right)$

$\sigma_{0} f(z)=\dot{\gamma}(z)$, and between parallel plates the local shear rate $\dot{\gamma}(z)=\frac{\partial v(z)}{\partial z}$, so that integration over $z$ gives:

$v(z)=\sigma_{0} f_{\text {bulk }} z+\sigma_{0}\left(f_{z=0}-f_{\text {bulk }}\right) \xi \sinh \left(\frac{z}{\xi}\right)+B \xi \cosh \left(\frac{z}{\xi}\right)+C$

Condition 2 from $z=0: C=v(0)-B \xi$, so that:

$v(z)=v(0)+\sigma_{0} f_{\text {bulk }} z+\sigma_{0}\left(f_{z=0}-f_{\text {bulk }}\right) \xi \sinh \left(\frac{z}{\xi}\right)+B \xi\left[\cosh \left(\frac{z}{\xi}\right)-1\right]$

Condition 3 from $z=h$ :

$B=\frac{v(h)-v(0)-\sigma_{0} f_{\text {bulk }} h-\sigma_{0}\left(f_{z=0}-f_{\text {bulk }}\right) \xi \sinh \left(\frac{h}{\xi}\right)}{\xi\left[\cosh \left(\frac{h}{\xi}\right)-1\right]}$

So that the velocity profile is:

$v(z)=v(0)+\sigma_{0} f_{\text {bulk }} z+\sigma_{0}\left(f_{z=0}-f_{\text {bulk }}\right) \xi \sinh \left(\frac{z}{\xi}\right)+\frac{v(h)-v(0)-\sigma_{0} f_{\text {bulk }} h-\sigma_{0}\left(f_{z=0}-f_{\text {bulk }}\right) \xi \sinh \left(\frac{h}{\xi}\right)}{\cosh \left(\frac{h}{\xi}\right)-1}\left[\cosh \left(\frac{z}{\xi}\right)-1\right]$

In the absence of slip and if $h$ is the distance between the plates, $v(h)$ is equal to the velocity of the moving wall and $v(0)=0$. 


\section{S7.4. Apparent wall slip}

Experimental procedures. Experiments were performed on an Anton Paar Physica MCR301 rheometer equipped with a PP geometry with a diameter of $4 \mathrm{~cm}$. The gap size was calibrated from the viscosity of paraffin oil according to procedures described in refs (Kramer, Uhl, \& Prud'homme, 1987; Kravchuk \& Stokes, 2013). Strain controlled flow curves of BCMF/CMC $0.20 / 0.05 \mathrm{wt} \%$ were measured with gap widths of 1.1 and $0.8 \mathrm{~mm}$, after pre-shearing the sample at $200 \mathrm{~s}^{-1}$ for 3 minutes. The shear rate was decreased from $2 \times 10^{2}$ to $2 \times 10^{-3} \mathrm{~s}^{-1}$ over the course of $1 \mathrm{~h}$.

Results and Discussion. The presence of (apparent) wall slip is determined by measuring flow curves in PP geometry at different plate separations. If we assume the apparent slip velocity $v_{\mathrm{s}}$ to be a function of stress alone, the apparent shear rate in parallel plates geometry has contributions from both the true shear rate and slippage: $\dot{\gamma}_{\text {app }}=\dot{\gamma}(\sigma)+\frac{2 v_{\mathrm{s}}(\sigma)}{d}$, where $d$ is the separation between the plates. We can determine $v_{s}(\sigma)$, by measuring the steady state flow curves $\dot{\gamma}_{d}(\sigma)$ at two different gap widths $d$ (Figure S7.7, for BCMF/CMC 0.20/0.05 wt\%). In this depiction we indeed observe minor but significant differences between the flow curves. The slip velocity follows from $v_{\mathrm{S}}(\sigma)=\frac{\dot{\gamma}_{d_{1}}(\sigma)-\dot{\gamma}_{d_{2}}(\sigma)}{2\left(1 / d_{1}-1 / d_{2}\right)}$ (Yoshimura \& Prud'homme, 1988). We observe apparent wall slip throughout a significant part of the flow curves, also at shear rates higher than the critical shear rate.

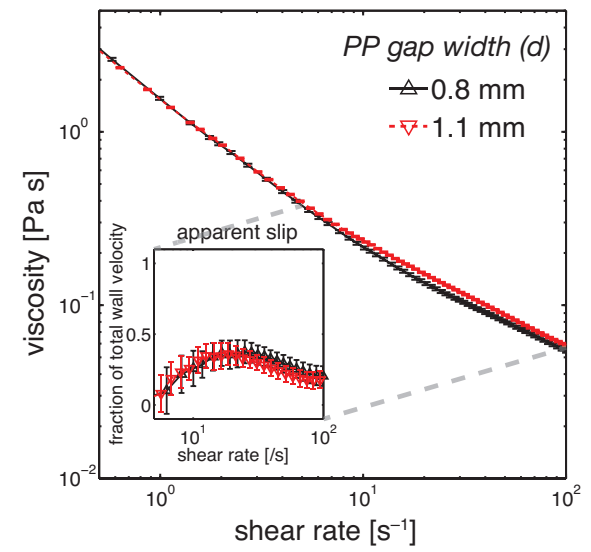

Figure S7.7: Gap-dependent viscosity differences in BCMF/CMC 0.20/0.05 wt $\%$. The flow curves were measured under strain-controlled conditions over the course of $1 \mathrm{~h}$. The contribution of the apparent slip to the total wall velocity (inset) is given by $v_{\mathrm{s}}(\sigma) /\left(d \dot{\gamma}_{d}(\sigma)\right)$. 


\section{References}

Alsteens, D., Dupres, V., Yunus, S., Latgé, J.-P., Heinisch, J. J., \& Dufrêne, Y. F. (2012). High-Resolution Imaging of Chemical and Biological Sites on Living Cells Using Peak Force Tapping Atomic Force Microscopy. Langmuir, 28(49), 16738-16744. http://doi.org/10.1021/la303891j

Barnes, H. A. (1995). A review of the slip (wall depletion) of polymer solutions, emulsions and particle suspensions in viscometers: its cause, character, and cure. Journal of Non-Newtonian Fluid Mechanics, 56(3), 221-251. http://doi.org/10.1016/0377-0257(94)01282-M

Bourouina, N., de Kort, D. W., Hoeben, F. J. M., Janssen, H. M., Van As, H., Hohlbein, J., et al. (2015). Complex Coacervate Core Micelles with Spectroscopic Labels for Diffusometric Probing of Biopolymer Networks. Langmuir, 31(46), 12635-12643. http://doi.org/10.1021/acs.langmuir.5b03496

Bouzid, M., Trulsson, M., Claudin, P., Clément, E., \& Andreotti, B. (2013). Nonlocal Rheology of Granular Flows across Yield Conditions. Physical Review Letters, 111(23), 238301-5. http://doi.org/10.1103/PhysRevLett.111.238301

Callaghan, P. T. (1993). Principles of Nuclear Magnetic Resonance Microscopy. New York: Oxford University Press.

Callaghan, P. T. (1999). Rheo-NMR: nuclear magnetic resonance and the rheology of complex fluids. Reports on Progress in Physics, 62(4), 599-670. http://doi.org/10.1088/0034-4885/62/4/003

Chaouche, M., \& Koch, D. L. (2001). Rheology of non-Brownian rigid fiber suspensions with adhesive contacts. Journal of Rheology, 45(2), 369-382. http://doi.org/10.1122/1.1343876

Cheng, D. C.-H. (1966). Cone-and-plate viscometry: explicit formulae for shear stress and shear rate and the determination of inelastic thixotropic properties. British Journal of Applied Physics, 17(2), 253-263. http://doi.org/10.1088/0508-3443/17/2/313

Chinga-Carrasco, G. (2011). Cellulose fibres, nanofibrils and microfibrils: The morphological sequence of MFC components from a plant physiology and fibre technology point of view. Nanoscale Research Letters, 6(1), 417. http://doi.org/10.1186/1556-276X-6-417

Cousins, S. (1995). Cellulose I microfibril assembly: computational molecular mechanics energy analysis favours bonding by van der Waals forces as the initial step in crystallization. Polymer, 36(20), 38853888. http://doi.org/10.1016/0032-3861(95)99782-P

Coussot, P., Nguyen, Q. D., Huynh, H. T., \& Bonn, D. (2002). Viscosity bifurcation in thixotropic, yielding fluids. Journal of Rheology, 46(3), 573-17. http://doi.org/10.1122/1.1459447

de Cagny, H., Fall, A., Denn, M. M., \& Bonn, D. (2015). Local rheology of suspensions and dry granular materials. Journal of Rheology, 59(4), 957-969. http://doi.org/10.1122/1.4919970

de Kort, D. W., Rombouts, W. H., Hoeben, F. J. M., Janssen, H. M., Van As, H., \& van Duynhoven, J. P. M. (2015a). Scaling Behavior of Dendritic Nanoparticle Mobility in Semidilute Polymer Solutions. Macromolecules, 48(20), 7585-7591. http://doi.org/10.1021/acs.macromol.5b01530

de Kort, D. W., van Duynhoven, J. P. M., Hoeben, F. J. M., Janssen, H. M., \& Van As, H. (2014). NMR nanoparticle diffusometry in hydrogels: enhancing sensitivity and selectivity. Analytical Chemistry, 86(18), 9229-9235. http://doi.org/10.1021/ac502211q

de Kort, D. W., van Duynhoven, J. P. M., Van As, H., \& Mariette, F. (2015b). Nanoparticle diffusometry for quantitative assessment of submicron structure in food biopolymer networks. Trends in Food Science \& Technology, 42(1), 13-26. http://doi.org/10.1016/j.tifs.2014.11.003

Derakhshandeh, B., Kerekes, R. J., Hatzikiriakos, S. G., \& Bennington, C. P. J. (2011). Rheology of pulp fibre suspensions: A critical review. Chemical Engineering Science, 66(15), 3460-3470. http://doi.org/10.1016/j.ces.2011.04.017

Goyon, J., Colin, A., \& Bocquet, L. (2010). How does a soft glassy material flow: finite size effects, non local rheology, and flow cooperativity. Soft Matter, 6(12), 2668-2678. http://doi.org/10.1039/c001930e

Goyon, J., Colin, A., Ovarlez, G., Ajdari, A., \& Bocquet, L. (2008). Spatial cooperativity in soft glassy flows. 
Nature, 454(7200), 84-87. http://doi.org/10.1038/nature07026

Haigler, C. H., White, A. R., Brown, R. M., Jr, \& Cooper, K. M. (1982). Alteration of in vivo cellulose ribbon assembly by carboxymethylcellulose and other cellulose derivatives. Journal of Cell Biology, 94(1), 6469. http://doi.org/10.1083/jcb.94.1.64

Iotti, M., Gregersen, Ø. W., Moe, S., \& Lenes, M. (2010). Rheological Studies of Microfibrillar Cellulose Water Dispersions. Journal of Polymers and the Environment, 19(1), 137-145. http://doi.org/10.1007/s10924-010-0248-2

Kamrin, K., \& Koval, G. (2012). Nonlocal Constitutive Relation for Steady Granular Flow. Physical Review Letters, 108(17), 178301-6. http://doi.org/10.1103/PhysRevLett.108.178301

Karppinen, A., Saarinen, T., Salmela, J., Laukkanen, A., Nuopponen, M., \& Seppälä, J. (2012). Flocculation of microfibrillated cellulose in shear flow. Cellulose, 19(6), 1807-1819. http://doi.org/10.1007/s10570012-9766-5

Klemm, D., Kramer, F., Moritz, S., Lindström, T., Ankerfors, M., Gray, D., \& Dorris, A. (2011). Nanocelluloses: A New Family of Nature-Based Materials. Angewandte Chemie-International Edition, 50(24), 5438-5466. http://doi.org/10.1002/anie.201001273

Kramer, J., Uhl, J. T., \& Prud'homme, R. K. (1987). Measurement of the viscosity of guar gum solutions to 50,000 s? 1 using a parallel plate rheometer. Polymer Engineering and Science, 27(8), 598-602. http://doi.org/10.1002/pen.760270811

Kravchuk, O., \& Stokes, J. R. (2013). Review of algorithms for estimating the gap error correction in narrow gap parallel plate rheology. Journal of Rheology, 57(2), 365-375. http://doi.org/10.1122/1.4774323

Kuijk, A., Koppert, R., Versluis, P., van Dalen, G., Remijn, C., Hazekamp, J., et al. (2013). Dispersions of Attractive Semiflexible Fiberlike Colloidal Particles from Bacterial Cellulose Microfibrils. Langmuir, 29(47), 14356-14360. http://doi.org/10.1021/la403397d

Lavoine, N., Desloges, I., Dufresne, A., \& Bras, J. (2012). Microfibrillated cellulose - Its barrier properties and applications in cellulosic materials: A review. Carbohydrate Polymers, 90(2), 735-764. http://doi.org/10.1016/j.carbpol.2012.05.026

Li, T. Q., Seymour, J. D., Powell, R. L., \& McCarthy, M. J. (1994). Visualization of flow patterns of cellulose fiber suspensions by NMR imaging. AIChE Journal, 40(8), 1408-1411. http://doi.org/10.1002/aic.690400812

Lin, D., Lopez-Sanchez, P., Li, R., \& Li, Z. (2014). Production of bacterial cellulose by Gluconacetobacter hansenii CGMCC 3917 using only waste beer yeast as nutrient source. Bioresource Technology, 151, 113-119. http://doi.org/10.1016/j.biortech.2013.10.052

Lowys, M. P., Desbrieres, J., \& Rinaudo, M. (2001). Rheological characterization of cellulosic microfibril suspensions. Role of polymeric additives. Food Hydrocolloids, 15(1), 25-32. http://doi.org/10.1016/S0268-005X(00)00046-1

Martoïa, F., Perge, C., Dumont, P. J. J., Orgéas, L., Fardin, M. A., Manneville, S., \& Belgacem, M. N. (2015). Heterogeneous flow kinematics of cellulose nanofibril suspensions under shear. Soft Matter, 11(24), 4742-4755. http://doi.org/10.1039/c5sm00530b

Møller, P. C. F., Mewis, J., \& Bonn, D. (2006). Yield stress and thixotropy: on the difficulty of measuring yield stresses in practice. Soft Matter, 2(4), 274-10. http://doi.org/10.1039/b517840a

Møller, P. C. F., Rodts, S., Michels, M. A. J., \& Bonn, D. (2008). Shear banding and yield stress in soft glassy materials. Physical Review E, 77(4), 041507. http://doi.org/10.1103/PhysRevE.77.041507

Nechyporchuk, O., Belgacem, M. N., \& Pignon, F. (2014). Rheological properties of micro-/nanofibrillated cellulose suspensions: Wall-slip and shear banding phenomena. Carbohydrate Polymers, 112, 432-439. http://doi.org/10.1016/j.carbpol.2014.05.092

Nichol, K., Zanin, A., Bastien, R., Wandersman, E., \& van Hecke, M. (2010). Flow-Induced Agitations Create a Granular Fluid. Physical Review Letters, 104(7), 078302-4. http://doi.org/10.1103/PhysRevLett.104.078302 
Ovarlez, G., Cohen-Addad, S., Krishan, K., \& Goyon, J. (2013). On the existence of a simple yield stress fluid behavior. Journal of Non-Newtonian Fluid Mechanics, 193, 68-79. http://doi.org/10.1016/j.jnnfm.2012.06.009

Ovarlez, G., Rodts, S., Chateau, X., \& Coussot, P. (2009). Phenomenology and physical origin of shear localization and shear banding in complex fluids. Rheologica Acta, 48(8), 831-844. http://doi.org/10.1007/s00397-008-0344-6

Paredes, J., Shahidzadeh, N., \& Bonn, D. (2015). Wall slip and fluidity in emulsion flow. Physical Review E, 92(4), 042313. http://doi.org/10.1103/PhysRevE.92.042313

Saarikoski, E., Saarinen, T., Salmela, J., \& Seppälä, J. (2012). Flocculated flow of microfibrillated cellulose water suspensions: an imaging approach for characterisation of rheological behaviour. Cellulose, 19(3), 647-659. http://doi.org/10.1007/s10570-012-9661-0

Saarinen, T., Haavisto, S., Sorvari, A., Salmela, J., \& Seppälä, J. (2014). The effect of wall depletion on the rheology of microfibrillated cellulose water suspensions by optical coherence tomography. Cellulose, 21(3), 1261-1275. http://doi.org/10.1007/s10570-014-0187-5

Seymour, J. D., Maneval, J. E., McCarthy, K. L., McCarthy, M. J., \& Powell, R. L. (1993). NMR velocity phase encoded measurements of fibrous suspensions. Physics of Fluids A, 5(11), 3010. http://doi.org/10.1063/1.858709

Siró, I., \& Plackett, D. (2010). Microfibrillated cellulose and new nanocomposite materials: a review. Cellulose, 17(3), 459-494. http://doi.org/10.1007/s10570-010-9405-y

Stejskal, E. O., \& Tanner, J. E. (1965). Spin diffusion measurements: Spin echoes in the presence of a timedependent field gradient. The Journal of Chemical Physics, 42, 288-292. http://doi.org/10.1063/1.1695690

Veen, S. J., Kuijk, A., Versluis, P., Husken, H., \& Velikov, K. P. (2014). Phase transitions in cellulose microfibril dispersions by high-energy mechanical deagglomeration. Langmuir, 30(44), 13362-13368. http://doi.org/10.1021/la502790n

Veen, S. J., Versluis, P., Kuijk, A., \& Velikov, K. P. (2015). Microstructure and rheology of microfibrilpolymer networks. Soft Matter, 1-6. http://doi.org/10.1039/C5SM02086G

Yamamoto, H., \& Horn, F. (1994). In Situ crystallization of bacterial cellulose I. Influences of polymeric additives, stirring and temperature on the formation celluloses $I_{\alpha}$ and $I_{\beta}$ as revealed by cross polarization/magic angle spinning (CP/MAS) ${ }^{13} \mathrm{C}$ NMR spectroscopy. Cellulose, 1(1), 57-66. http://doi.org/10.1007/BF00818798

Yan, H., Lindström, T., \& Christiernin, M. (2006). Some ways to decrease fibre suspension flocculation and improve sheet formation. Nordic Pulp \& Paper Research Journal, 21(01), 036-043. http://doi.org/10.3183/NPPRJ-2006-21-01-p036-043

Yoshimura, A., \& Prud'homme, R. K. (1988). Wall Slip Corrections for Couette and Parallel Disk Viscometers. Journal of Rheology, 32(1), 53-67. http://doi.org/10.1122/1.549963 


\section{8}

General discussion 


\subsection{Introduction}

In the previous chapters, we have explored the use of diffusional nanoparticles to quantify submicron structural length scales and heterogeneity in polymer gels and solutions. We described designs of spectroscopically labeled nanoparticles and diffusometric methodology (both fluorescence and magnetic resonance-based). We then applied the novel particles in three model systems: sub-micron-heterogeneous $\kappa$-carrageenan gels, homogeneous poly(ethylene glycol) (PEG) solutions, and homogenized cellulose microfibril dispersions. A complementary rheological and rheo-MRI velocimetry study of the last model system revealed persistent heterogeneity on the micron scale. This last chapter will critically review this work, and provide an outlook for possible future applications of the methodology developed therein.

\subsection{Nanoparticle diffusometry}

Chapter 2 provided an elaborate review on existing methodology and theory that has been used to describe nanoparticle diffusion in polymer gels and solutions. In this context, we also identified two new developments for the application of nanoparticle diffusometry in foods, which typically are complex mixtures of (bio)polymers and small-molecular solutes in water. The first development was the design and manufacturing of novel functionalized nanoparticles, and the second was the use of diffusional nanoparticles for detection of sub-micron heterogeneity in biopolymer hydrogels.

\subsubsection{Novel nanoparticle designs}

Besides the fact that only a handful of spectroscopic methods are available to observe self-diffusion of nanoparticles with diameters in the tens-of-nanometers range, all of them require labeling of the particles to allow their observation in complex matrices. An overview of all particles that were introduced in the previous chapters is presented in Figure 8.1. These particles adhere to three design rules, namely that (1) the particles are spherical to avoid complex diffusion modes such as reptation, (2) the particles have a PEG corona to counter specific interactions between the particles and the polymer matrix, and (3) spectroscopic labels are positioned internally, for the same reason. Particles with complex coacervate cores (C3M) have hydrodynamic diameters of $\sim 30 \mathrm{~nm}$, while those with poly(propylene imine) (PPI) dendritic cores are more compact with diameters between $3 \mathrm{~nm}$ (G1) and 11 (G5+spacer) nm. Labels include ATTO-488 fluorescent dyes, ${ }^{19} \mathrm{~F}$ moieties, and TEMPO spin labels. This set of particles enables the use of diffusometric methods such as PFG NMR diffusometry $\left({ }^{1} \mathrm{H}\right.$ and $\left.{ }^{19} \mathrm{~F}\right)$, fluorescence recovery after photobleaching (FRAP), fluorescence correlation spectroscopy (FCS), total internal reflection (TIRF) fluorescent particle tracking, raster image correlation spectroscopy (RICS) and Overhauser dynamic nuclear polarization (ODNP)-enhanced NMR spectroscopy . 

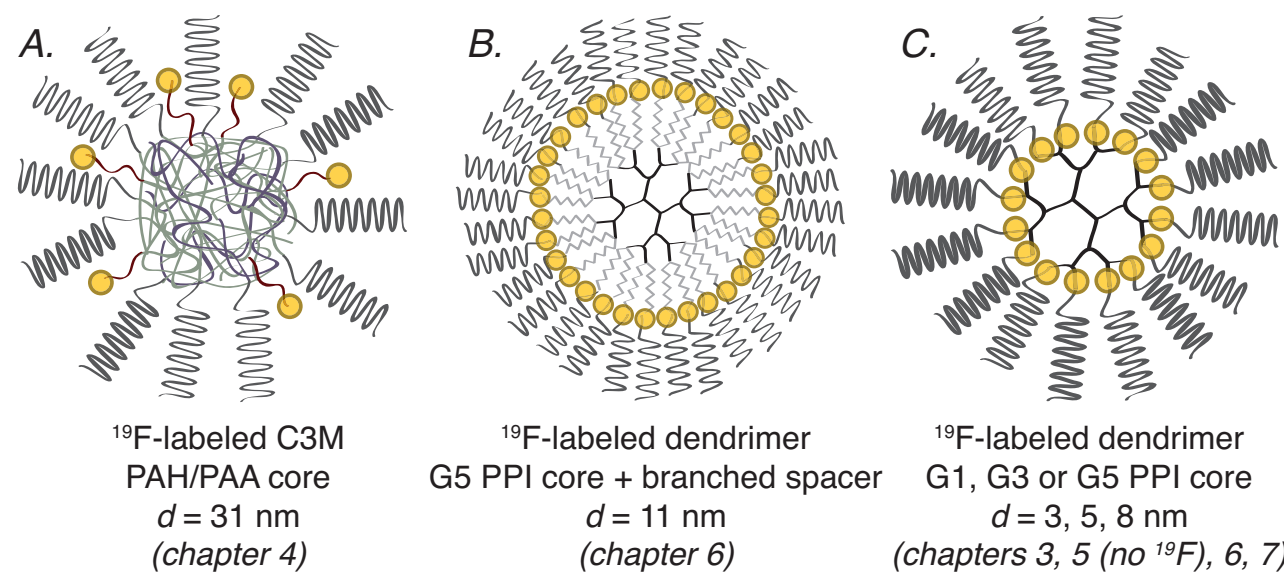

${ }^{19} \mathrm{~F}$-labeled dendrimer

G5 PPI core + branched spacer $d=11 \mathrm{~nm}$ (chapter 6)
${ }^{19} \mathrm{~F}$-labeled dendrimer
G1, G3 or G5 PPI core $d=3,5,8 \mathrm{~nm}$ (chapters 3, 5 (no $\left.{ }^{19} F\right), 6,7$ )
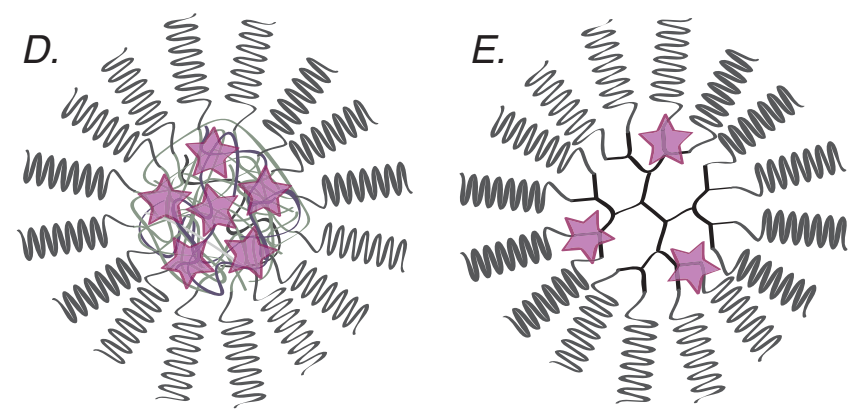
ATTO-labeled C3M PAH/PMAA core $d=26 \mathrm{~nm}$ (chapter 4)

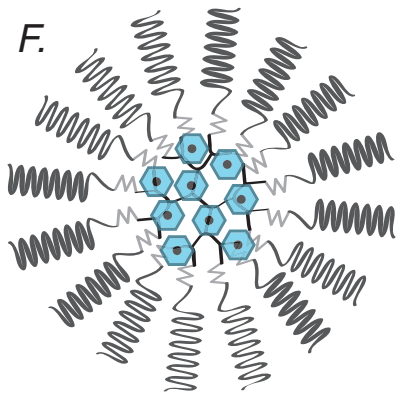

TEMPO-labeled dendrimer G5 PPI core + spacer $d=10 \mathrm{~nm}$ (chapter 5)

Figure 8.1: Overview of all nanoparticle designs used in the previous chapters and their hydrodynamic diameters $d$. (A) ${ }^{19}$ F-labeled, PEGylated C3M, (B) ${ }^{19}$ F-labeled, PEGylated dendrimer with a G5 PPI core and an additional branched spacer, $(C){ }^{19}$ F-labeled, PEGylated dendrimer with a G1, G3, or G5 PPI core, (D) ATTO-488-labeled, PEGylated C3M, (E) ATTO-488-labeled PEGylated dendrimer with a G5 PPI core, (F) TEMPO-labeled, PEGylated dendrimer with a G5 PPI core and an additional spacer.

\subsubsection{Diffusometric methodology}

Physical models of diffusion. We have demonstrated the use of each of these diffusometric methods, and the way in which they complement each other. PFG NMR diffusometry, for example, provides the ensemble average propagator, a bulk property from which self-diffusion coefficients can be calculated, while RICS and TIRF particle tracking allow measurements of self-diffusion 
coefficients of individual particles. Self-diffusion coefficients as measured by these methods can be used to calculate average network properties via physical models of diffusion, as outlined in chapter 2 . The problem with such models, however, is that a priori assumptions have to be made regarding the microstructure of the gel (e.g., network flexibility and degree of heterogeneity), to justify the choice of model. These assumptions are typically based on observations by highresolution microscopy methods, of which the limitations in resolution, and the necessary invasive sample preparation steps, were the motives to revert to nanoparticle diffusometry in the first place. For this reason, the use of physical models of diffusion is limited to the calculation of average properties to within an error margin that is difficult to quantify. On the other hand, one could potentially use a number of nanoparticles with different sizes in the same model system, and see whether the degree of retardation for the differently sized particles is consistent for a certain model or parameter set. If so, this would be an indication that the chosen model could be appropriate to describe the system.

Probing sub-micron structural heterogeneity. The work presented in this thesis explored a possibility to circumvent the problems with physical models of diffusion, by focusing not so much on the determination of absolute network parameters, but instead on the observation of network heterogeneity as apparent from non-Gaussian diffusion. Such effects were first observed in $\kappa$ carrageenan gels by Lorén et al. using dendrimer nanoparticles and PFG NMR diffusometry (Lorén et al., 2009b). Due to its ability to measure the ensemble-average propagator at welldefined observation times, PFG NMR diffusometry is ideally suited to detect and quantify nonGaussian diffusion. This in principle would allow a similar approach as taken by Valentine et al. to investigate the microstructure of heterogeneous soft materials by tracking fluorescent particles with diameters of $500 \mathrm{~nm}$ (Valentine et al., 2001). PFG NMR diffusometry, however, does not offer an equally broad time window as the video-particle tracking method employed in that work (less than orders of magnitude for PFG NMR, versus three orders of magnitude for the particle tracking method). Moreover, the dynamics of nanoparticles are much faster than those of the much larger ( $>50$ times) particles used by Valentine et al. This means that the time window where non-Gaussian effects are expected for nanoparticles is at much shorter time scales than accessible by PFG NMR diffusometry: If network heterogeneity occurs at the sub-micron scale (e.g., $10^{2}$ $\mathrm{nm}$ ), and the nanoparticle diffusion coefficient is $\sim 5 \times 10^{-11} \mathrm{~m}^{2} / \mathrm{s}$ (as for the G5 dendritic particles), then we expect non-Gaussian diffusion at a time scale of $0.1 \mathrm{~ms}$, two decades shorter than the $10-10^{2} \mathrm{~ms}$ time scale accessible by PFG NMR diffusometry. Beyond the $0.1 \mathrm{~ms}$ time scale, we in principle expect to observe Gaussian diffusion because the effects of heterogeneity will have averaged out (Lorén, Nydén, \& Hermansson, 2009a). Because of this reason, the observation of non-Gaussian diffusion in $\kappa$-carrageenan gels was partly a mystery, which was solved when it became clear that there existed mobile and immobile particle fractions, pointing at the presence of small pores in which a fraction of the nanoparticles was trapped. 
Before looking at the trapping effect in more detail, we first continue our discussion of methodology for probing sub-micron structural heterogeneity. Besides PFG NMR diffusometry to measure the ensemble-average propagator, TIRF single particle tracking has the potential to do the same. For this method, however, the time window turns out to be even more narrow: the currently achievable frame rate is one frame per $\sim 5 \mathrm{~ms}$, while the particles, due to their fast dynamics, remain within the field only for a few frames (cf. chapter 4). This does not help to solve the issues pointed out for PFG NMR above. Alternatively, FRAP is able to determine multiple diffusion coefficients. But for this method, the underlying assumption is that these diffusion coefficients are time-independent (as would be the case, for instance, for two diffusing species with different sizes in neat solvent). Secondly, because the bleaching spot is micron-size, and the determination of diffusion coefficients relies on averaging over the bleaching spot, the method cannot be sensitive to sub-micron heterogeneity.

Measuring time-dependent diffusion coefficients. Alternatively, instead of looking for signs of nonGaussian propagators, observation of the time-dependent mean particle diffusion coefficient could have been helpful. For larger particles, this information can be used to calculate viscoelastic properties of gels and solutions (Mason \& Weitz, 1995), but for nanoparticles that are smaller than the microscopic features of gels, a similar approach could potentially be taken to probe pore sizes in heterogeneous polymer gels: In polymer gels, we would expect that the diffusivity of the nanoparticles changes significantly at time scales where the mean displacement is comparable to the size of the interstitials. For polymer solutions, where the relaxation rates of the polymers are not as high as in gels, and for which dynamics are a more meaningful concept than structure, it is more convenient to assess the dynamics of the polymers themselves instead of those of embedded nanoparticles. PFG NMR (Stepišnik, Lahajnar, Zupančič, \& Mohoric, 2013) and field cycling NMR (Hofmann et al., 2015) have recently been shown to be powerful tools to this end.

Only a limited number of methods is potentially able to probe time-dependent nanoparticle diffusion coefficients in polymer gels. Stepišnik and Callaghan have shown that time-dependent diffusion coefficients can be obtained by using a series of inversion RF pulses in the presence of a constant background gradient $G$ whose effect is inverted by the successive pulses; This approach is known as the modulated gradient spin echo (MGSE) experiment (Stepišnik \& Callaghan, 2001). The attenuation of the echo intensity $E$ after $N$ inversion pulses due to the presence of a field gradient with amplitude $G$ amounts to $E[N T, \omega]=\exp \left(-8 \gamma^{2} G^{2} D(\omega) N T /\left(\omega^{2} \pi^{2}\right)\right)$, where $\gamma$ is the gyromagnetic ratio, and $D(\omega)$ the diffusion coefficient at modulation frequency $\omega=2 \pi / T$ (where $T$ is the time between the inversion pulses) (Lasič, Stepišnik, Mohorič, Serša, \& Planinšič, 2007). This experiment is potentially able to access the time regime of interest (at $T \sim 0.1 \mathrm{~ms}$ ). However, the consequence of the very small displacements that occur for our nanoparticles during such small time intervals, in combination with the relatively low $T_{2}$ times associated with the nanoparticles that limit the total experimental time $N T$, is that the signal attenuation $E$ is $<0.1 \%$ if a 
gradient of $1 \mathrm{~T} / \mathrm{m}$ is applied for $500 \mathrm{~ms}$ (for G5 nanoparticles in a ${ }^{1} \mathrm{H}$ experiment). This precludes the use of the MGSE method in this context until the use strong field gradients $(\sim 10 \mathrm{~T} / \mathrm{m})$ for prolonged time periods $(\sim 1 \mathrm{~s})$ becomes technically feasible, or until a setup is available that uses the magnetic gradient of a fringe field, but at the same offers adequate sensitivity.

Besides the MGSE experiment, FCS also allows measurement of the mean nanoparticle displacement over time, as has been demonstrated for fluorescent particles with diameters of $\sim 100$ $\mathrm{nm}$ in solutions of heavy PEG polymers (Rathgeber, Beauvisage, Chevreau, Willenbacher, \& Oelschlaeger, 2009). In this work, the mean square displacement of these particles could be determined over an impressive six orders of magnitude in time (up from microseconds). However, a similar approach did not work for the ATTO-labeled nanoparticles because of limited sensitivity at short time scales, and bleaching of ATTO dyes at longer time scales. This experiment could potentially work if intrinsically fluorescent nanoparticles (i.e., nanoparticles whose fluorescence cannot bleach) such as gold colloidal nanoparticles are used instead (Kohli \& Mukhopadhyay, 2012).

It is clear that there are still significant experimental challenges to be overcome before all information potentially available from self-diffusion of nanoparticles can be accessed. With current experimental methods, only long-time diffusion coefficients, where all information about submicron structural heterogeneity has averaged out, are available. An exception occurs in case particles become trapped in the smallest of the interstitials, in which case microstructural information is still directly accessible. That is, if the degree to which particles are trapped is a function of particle size, we can, in principle, probe the distribution of pore sizes by varying the particle size and measuring the fraction of immobilized particles.

Immobilization of nanoparticles in heterogeneous networks. In the previous chapters we have shown that in the $\kappa$-carrageenan model system, particles are indeed immobilized by the polymer network. This effect appears to be a function of $\kappa$-carrageenan concentration (Figure 4.9), nanoparticle size (cf. Figures 5.2, S5.2, and S5.3) and $\mathrm{Na}^{+}$concentration (Figure 5.3), which has been shown to influence heterogeneity in these gels (Hagman, Lorén, \& Hermansson, 2012; Hermansson, Eriksson, \& Jordansson, 1991; Lorén et al., 2009b). A plausible explanation of these effects is that the observed immobilization is a topological effect and that particles can be trapped in small interstitials. The hallmark of restricted self-diffusion of particles within small pores are diffraction phenomena in the PFG NMR attenuation curves (Callaghan, Coy, MacGowan, Packer, \& Zelaya, 1991). We did not observe such effects, which might be due to a large distribution in pore sizes leading to a loss of the diffraction phenomena (Shemesh, Özarslan, Basser, \& Cohen, 2010). On the other hand, we have also not yet been able to conclusively show (e.g., with microscopic images) that the nanoparticles are indeed trapped in nanoscopic interstitials, rather than otherwise immobilized by the polymer network, for instance by simple adsorption onto the polymer chains themselves. Although the C3Ms and the dendrimer nanoparticles were PEGylated as a measure to 
reduce the probability of attractive interactions between particles and polymers, there is still a possibility that at least some of the particles are, for example, not fully charge-neutral. For C3Ms, for instance, it is conceivable that the positively and negatively charged polyelectrolytes are not perfectly balanced. Although it is plausible that the particles are indeed trapped into small domains, the possibility of adsorption of particles to the polymer chains is not inconsistent with our data so that this question should be addressed in future work.

Besides concerns about the exact manner in which the particles are immobilized, there are also issues with the quantitative interpretation of the fraction of immobilized particles. An insightful experiment that used ${ }^{19} \mathrm{~F}$-labelded G5 particles in $2 \mathrm{wt} \% \kappa$-carrageenan at $0 \mathrm{mM} \mathrm{Na}^{+} / 20 \mathrm{mM} \mathrm{K}^{+}$, shed a different light on the apparent particle-size dependency of the relative fraction of trapped particles. Here, it turned out that the absolute magnitude of the immobilized fraction was independent of particle concentration. As shown in Figure 8.2, by doubling the concentration of nanoparticles, the absolute amplitude of the NMR signal increased proportionally by a factor of two, whereas the amplitude of the immobilized fraction (the tail of the attenuation curve at high $b$ values) remained of identical amplitude.

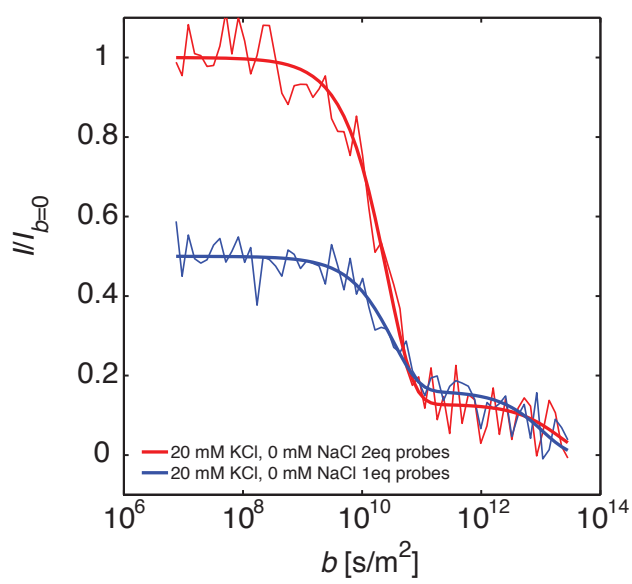

Figure 8.2: $\mathrm{PFG}{ }^{19} \mathrm{~F}$ NMR diffusometry (stimulated echo, observation time $200 \mathrm{~ms}$ ) attenuation curves of ${ }^{19} \mathrm{~F}$ labeled G5 dendritic nanoparticles in $2 \mathrm{wt} \% \mathrm{\kappa}$-carrageenan at $0 \mathrm{mM} \mathrm{Na}^{+} / 20 \mathrm{mM} \mathrm{K}{ }^{+}$. The upper, red curve was measured at a nanoparticle concentration that was twice higher than the concentration used to measure the lower, blue curve. The attenuation curves are fitted to bi-exponential StejskalTanner curves. The use of ${ }^{19} \mathrm{~F}$ measurements allowed us to start the experiments at low $b$-values of $\sim 10^{-7}$ $\mathrm{s} / \mathrm{m}^{2}$ (where ${ }^{1} \mathrm{H}$ measurements would suffer from significant background signal of water), so that no back-prediction was needed to estimate the signal amplitude at lower $b$-values.

This finding does not contradict the theory of particles being trapped in small interstitials. It might very well be that the number of small interstitials is limited and that these pores are fully saturated 
at $0.1 \mathrm{wt} \%$ particle concentration. It does introduce a problem, however, for the experiments presented in chapter 5 that used G1, G3, and G5 dendrimers in $\kappa$-carrageenan at different $\mathrm{Na}^{+}$ concentrations (Figures 5.2, S5.2, and S5.3), but at an identical particle weight fraction for all particles. Due to the difference in molecular weight between the particles, the molar concentration of G1 particles is significantly higher than that of G5 particles. At higher particle concentrations, in case all small pores are saturated, the relative amplitude of the fraction of trapped particles will appear smaller. This means, in retrospect, that it would make more sense to use an identical molar particle concentration between experiments, although this would lead to a significant loss of signal intensity for the smaller particles. The issue also makes a direct comparison between different diffusometric methods difficult, because of differences in sensitivity between the methods and hence the necessity to use significantly different particle concentrations.

Viscosity of the water phase. In chapter 5, by using ODNP-enhanced NMR spectroscopy, we demonstrated that water diffusivity in the immediate vicinity of trapped nanoparticles is lower than water diffusivity surrounding freely diffusing particles. This supported the idea of immobilization of the nanoparticles in small pores (although adsorption onto the polymer chains would lead to a similar observation).

Regardless of the exact physical origin of the immobilization effect as observed by PFG NMR diffusometry, and the meaning of the relative fractions of mobile and immobile nanoparticles, the ODNP NMR method is uniquely able to probe the structure and viscosity of the water phase in gels. Although chapter 5 only focused on the question whether immobilized particles are situated in more viscous surroundings than mobile particles, we can also access information about the viscosity of water in the "bulk" of larger interstitials. To this end, we need to reference the data on local water retardation in the vicinity of mobile TEMPO-labeled nanoparticles (Figure 5.6) against the effects measured for freely dissolved TEMPO spin labels (i.e., TEMPO not attached into nanoparticles).

We performed such experiments at a fixed salt concentration of $200 \mathrm{mM} \mathrm{Na}^{+}$at different $\kappa$ carrageenan concentrations between 1-5 wt\%. Besides the TEMPO-functionalized dendrimers, we used different small molecule spin probes with varying functional pendant groups (4-hydroxyTEMPO, TEMPO and 4-amino-TEMPO) to test for the effects of attractive or repulsive interactions between spin probe and the $\kappa$-carrageenan strands.

In general, the size of the TEMPO-dendrimers may be of the same order of magnitude in size as the interstitials of the gel, especially in $5 \mathrm{wt} \% \mathrm{\kappa}$-carrageenan gels, so that the local water dynamics around mobile dendrimers may be already perturbed by their interaction with the gel network or their hydration water whose viscosity is increased. In contrast, the size of small molecule TEMPO is much smaller than the gel strand network, so its self-diffusion should be relatively unhindered. Here, the question is whether the bulk solvent viscosity, as reflected in the local solvent diffusivity, is bulk-like within the gel interstitials or already altered by the gel-water interaction that affects the 
water hydrogen-bond network of the local solvent. The freely dissolved TEMPO spin probes report whether the local solvent property within the gel interstitial is bulk-like or not.

The results are presented in Figure 8.3. Generally, we found that the water diffusion in the gel interstitials was retarded up to $\sim 30 \%$ at $5 \%$ polymer concentration. These results suggest that the previously observed retardation for the dendrimer diffusion may not only be a result of percolation effects within the gel, but also a results of lower water mobility (i.e., increased local water viscosity). This apparent retardation of the bulk water diffusion coefficient would not be expected from a picture where adjacent $\kappa$-carrageenan strands are at least tens-of-nanometers apart, because the layers with (more viscous) hydration water that surround the polymer strands should not stretch that far into the liquid (Ramadugu, Chung, Xia, \& Margulis, 2009). This might mean that more flexible polymer strands are present within the apparent bulk volume of the pores, leading to an increase in the amount of hydration water while still allowing the nanoparticles to diffuse. This would also violate the validity of physical models of diffusion, which are often based on a more straightforward microstructural picture. More specific measurements are needed to confirm the presence of flexible polymer strands in the bulk of the pores.

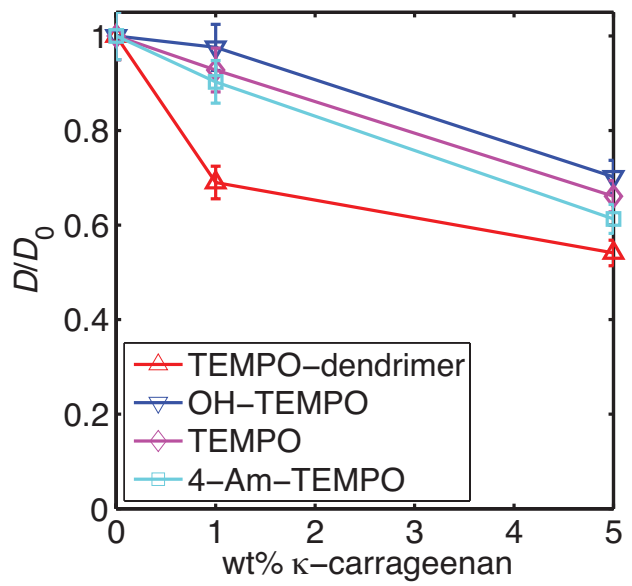

Figure 8.3: Retardation of local water in $\kappa$-carrageenan gels as observed by ODNP-enhanced NMR spectroscopy using four different spin probes. $D_{0}$ is the water diffusion coefficient as determined by this method in water without polymer. Experiments were performed in $200 \mathrm{mM} \mathrm{Na}^{+} / 20 \mathrm{mM} \mathrm{K}^{+}$gels.

While the freely dissolved spin probes distribute truly homogeneously over the available volume, the dendritic particles are more likely to probe more retarded water close to the $\kappa$-carrageenan strands, due to skewed partitioning because of the immobilization effect. The observed difference between freely dissolved TEMPO and dendrimer-TEMPO is larger at $1 \mathrm{wt} \%$ than at $5 \mathrm{wt} \% \mathrm{k}$ carrageenan, possibly because at $5 \mathrm{wt} \%$, the polymer strands are close enough together for the hydration water layers of adjacent polymer strands to overlap, decreasing the bias for probing 
hydration water for the dendrimer particles over the freely dissolved spin probes.

Future developments. In chapter 2, we reviewed the traditional application of nanoparticle diffusometry that mainly uses physical models of diffusion to extract structural parameters from (bio)polymer hydrogels. In the section above, we concluded that the application of physical models of diffusion to extract network parameters suffers from the shortcoming that microscopic input is needed to make an appropriate choice of diffusion model. Promising approaches that do not rely on physical models of diffusion can be based on direct observation of micron-scale heterogeneity by methods such as PFG NMR diffusometry, ODNP-enhanced NMR spectroscopy, and FRAP CLSM. The ODNP effects observed for low-molecular weight paramagnetic probes may complement the information that can be obtained by TEMPO-labeled nanoparticles.

Besides this "quantitative" microstructural approach, a simpler, but perhaps equally exciting model-free application of nanoparticle diffusometry is to use it as a tool to study biopolymer networks under chemical and physical processing conditions. In casein systems, for instance, Le Feunteun et al. have monitored the evolution of self-diffusion coefficients of PEG nanoparticles as a function of $\mathrm{pH}$ during acid coagulation, during chymosin-induced coagulation and during combined acid-enzyme coagulation. PEG diffusion revealed many of the structural changes occurring during the coagulation process, which is an essential step in cheese production. Such changes could be correlated to changes in the elasticity of the material as determined by rheology (Le Feunteun \& Mariette, 2008b; 2008a). Besides monitoring gelation processes, other structural transitions can be monitored in a time-dependent and non-invasive manner at sub-micron scale, such as syneresis and retrogradation. Particularly with the new set of complementary nanoparticles described in this thesis, such applications, which are highly relevant for industrial processing and quality control, have the potential to become routinely applied in food process and product development.

\subsection{Rheo-MRI velocimetry}

Rheo-MRI is a powerful tool to study the flow behavior of yield stress fluids that might be thixotropic and/or structurally heterogeneous. Many foods fall into this broad category, and a study of shear flow profiles will help to understand the details of their rheology. In chapter 7, a study of the rheology of cellulose microfibril dispersions showed how this method can contribute to our understanding of structure-function relationships in foods. Therein, microscopic analysis indicated that the dispersions were homogeneous at the sub-micron scale, but a study of their flow behavior demonstrated persistent heterogeneity at the micron scale.

Although microstructural heterogeneities must be at the physical origin of the viscosity variations across the gap in cone-and-plate (CP) geometry (Figure 7.4), and the nonlocal model appears to describe these viscosity variations adequately, more rheological tests are needed to unequivocally demonstrate that either migration effects or confinement effects underlie the nonlocality. Strictly 
speaking, nonlocal effects imply that the apparent viscosity of the fluid does not correspond to that found for flow at a larger scale, which is different from the viscosity simply being different in two different places within the same flow when the stress is homogeneous (as in the CP experiments), whatever the flow size. Goyon et al., for instance, compared local flow curves measured in (narrow) capillaries to those measured in a (much larger) concentric cylinders (CC) geometry (Goyon, Colin, Ovarlez, Ajdari, \& Bocquet, 2008). Construction of local flow curves is not possible in CP geometry due to the uniform stress value across the gap. Given the relatively large size of the heterogeneities in our cellulose system, we should therefore ideally measure and compare local flow curves in two CC geometries with significantly different gap widths. This would require us to scale up our experiment from the very compact 7T rheo-MRI setup used in chapter 7 (the diameter of the outer cylinder is $22 \mathrm{~mm}$ ) to a much larger MRI facility. In parallel, for a proper comparison of the local rheology in both the smaller and the larger setup, bulk flow curves should be measured on a standard rheological instrument of the same dimensions. Only in case the nonlocal effects observed in the smaller rheo-MRI setup disappear in the larger setup, we can conclude that confinement effects must be the cause of the nonlocality (Goyon et al., 2008).

Alternatively, some heterogeneity of concentration of the elements in the fluid might be at the origin of the effects observed in CP geometry (i.e., Figure 7.4). To investigate this possibility in more detail, we measured flow profiles of BCMF/CMC dispersions in CC geometry, as shown in Figure 8.4.

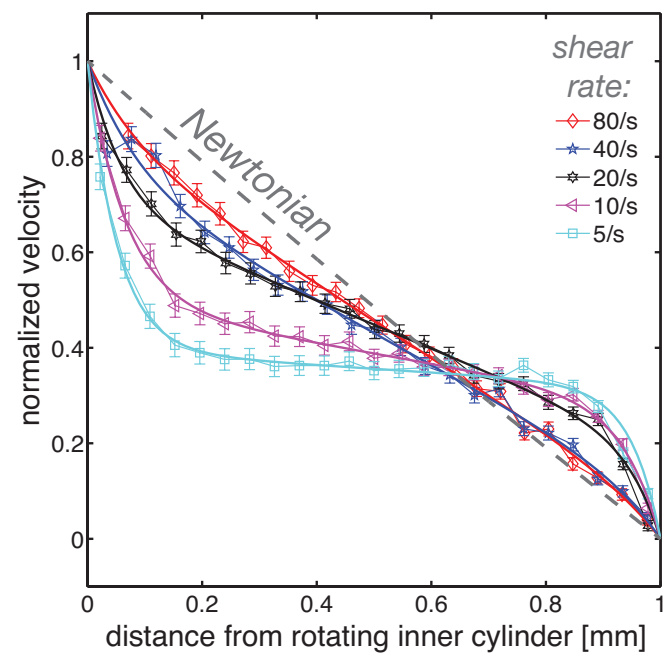

Figure 8.4: BCMF/CMC 0.2/0.05 wt $\%$ dispersions in concentric cylinders geometry. The diameter of the inner cylinder is $17 \mathrm{~mm}$ and the gap width is $1.0 \mathrm{~mm}$.

The flow profiles are different from those expected for typical yield-stress fluids. The stress at the 
outer cylinder is $20 \%$ lower than at the inner cylinder and decreases across the gap in a quadratic manner, the shear rate close to the outer cylinder increases sharply. This is similar to what was observed by Martoïa et al. (Martoïa et al., 2015) and Saarinen et al. (Saarinen, Haavisto, Sorvari, Salmela, \& Seppälä, 2014) for cellulose dispersions, which they attributed to wall depletion effects (i.e., a heterogeneity of concentration of suspended elements). On the other hand, these were systems without added CMC, which prevents aggregation of cellulose fibers, so that their observations are not directly applicable to BCMF/CMC dispersions (e.g., we do not have significant $T_{2}$ contrast in MRI images of sheared BCMF/CMC dispersions, as opposed to sheared BCMF dispersions without CMC, cf. Figure 7.1).

Future developments. Rheo-MRI velocimetry is an indispensable tool to understand the flow behavior of yield-stress fluids, particularly if they are heterogeneous and/or thixotropic. Our study of the shear flow behavior of cellulose dispersions has shown that heterogeneities dominate the flow behavior, and invoked the appropriate analytical framework to derive the size of these heterogeneities, but, as outlined above, there are still open questions.

In 1999, Callaghan pointed out the vast potential of rheo-MRI for the analysis of food materials (Callaghan, 1999), but there have been only a handful of studies on such systems, most notably on butter (Britton \& Callaghan, 2000). Over the last decade, however, our understanding of the rheology of (thixotropic) yield stress fluids has improved dramatically (Ovarlez et al., 2015). In this context, a rheo-MRI view could now help us improve our understanding of the rheology of protein-, lipid-, or polysaccharide-based gels or dispersions under processing conditions or during consumption.

Rheo-MRI and nanoparticle diffusometry experiments are both PFG NMR-based methods. This means that both experiments can be carried out in the same experimental setup, opening up possibilities to study the effects of shear on microstructure such as shear-induced network anisotropy. For example, in systems of aligned fibers, diffusion in the direction parallel to the fibers should be less hindered than in the orthogonal direction. Such effects have been observed for nanoparticle diffusion in aligned collagen fibers in cartilage by line-FRAP. (Stylianopoulos, DiopFrimpong, Munn, \& Jain, 2010) Although the observed effects were small even in this highly aligned system (nanoparticle self-diffusion coefficients in different directions vary only a few percent), this approach could potentially work e.g. to monitor the formation of aligned protein fibrils during shear flow (Manski, van der Goot, \& Boom, 2007). By using a flow-compensated scheme, Lutti and Callaghan showed that it is possible to measure particle diffusion also under dynamic (shear) conditions by MRI (Lutti \& Callaghan, 2006). This would open up the possibility to observe in situ the formation of sub-micron anisotropic food structures under shear. 


\section{References}

Britton, M. M., \& Callaghan, P. T. (2000). NMR Velocimetry Study of the Temperature Dependent Rheology of Butter, Semisoft Butter and Margarine. Journal of Texture Studies, 31(3), 245-255. http://doi.org/10.1111/j.1745-4603.2000.tb00288.x

Callaghan, P. T. (1999). Rheo-NMR: nuclear magnetic resonance and the rheology of complex fluids. Reports on Progress in Physics, 62(4), 599-670. http://doi.org/10.1088/0034-4885/62/4/003

Callaghan, P. T., Coy, A., MacGowan, D., Packer, K. J., \& Zelaya, F. O. (1991). Diffraction-like effects in NMR diffusion studies of fluids in porous solids. Nature, 351(6326), 467-469. http://doi.org/10.1038/351467a0

Goyon, J., Colin, A., Ovarlez, G., Ajdari, A., \& Bocquet, L. (2008). Spatial cooperativity in soft glassy flows. Nature, 454(7200), 84-87. http://doi.org/10.1038/nature07026

Hagman, J., Lorén, N., \& Hermansson, A.-M. (2012). Probe diffusion in $\kappa$-carrageenan gels determined by fluorescence recovery after photobleaching. Food Hydrocolloids, 29(1), 106-115. http://doi.org/10.1016/j.foodhyd.2012.02.010

Hermansson, A. M., Eriksson, E., \& Jordansson, E. (1991). Effects of potassium, sodium and calcium on the microstructure and rheological behaviour of kappa-carrageenan gels. Carbohydrate Polymers, 16(3), 297-320. http://doi.org/10.1016/0144-8617(91)90115-S

Hofmann, M., Gainaru, C., Cetinkaya, B., Vaiullin, R., Fatkullin, N., \& Roessler, E. A. (2015). Field-Cycling Relaxometry as a Molecular Rheology Technique: Common Analysis of NMR, Shear Modulus and Dielectric Loss Data of Polymers vs Dendrimers. Macromolecules, 48(20), 7521-7534. http://doi.org/10.1021/acs.macromol.5b01805

Kohli, I., \& Mukhopadhyay, A. (2012). Diffusion of Nanoparticles in Semidilute Polymer Solutions: Effect of Different Length Scales. Macromolecules, 45(15), 6143-6149. http://doi.org/10.1021/ma301237r

Lasič, S., Stepišnik, J., Mohorič, A., Serša, I., \& Planinšič, G. (2007). Autocorrelation spectra of an airfluidized granular system measured by NMR. Europhysics Letters (EPL), 75(6), 887-893. http://doi.org/10.1209/epl/i2006-10193-6

Le Feunteun, S., \& Mariette, F. (2008a). Effects of Acidification with and without Rennet on a Concentrated Casein System: A Kinetic NMR Probe Diffusion Study. Macromolecules, 41(6), 2079-2086. http://doi.org/10.1021/ma702248z

Le Feunteun, S., \& Mariette, F. (2008b). PFG-NMR Techniques Provide a New Tool for Continuous Investigation of the Evolution of the Casein Gel Microstructure after Renneting. Macromolecules, 41(6), 2071-2078. http://doi.org/10.1021/ma702246m

Lorén, N., Nydén, M., \& Hermansson, A.-M. (2009a). Determination of local diffusion properties in heterogeneous biomaterials. Advances in Colloid and Interface Science, 150(1), 5-15. http://doi.org/10.1016/j.cis.2009.05.004

Lorén, N., Shtykova, L., Kidman, S., Jarvoll, P., Nydén, M., \& Hermansson, A.-M. (2009b). Dendrimer diffusion in kappa-carrageenan gel structures. Biomacromolecules, 10(2), 275-284. http://doi.org/10.1021/bm801013x

Lutti, A., \& Callaghan, P. T. (2006). Measurement of diffusion in the presence of shear flow. Journal of Magnetic Resonance, 180(1), 83-92. http://doi.org/10.1016/j.jmr.2006.01.009

Manski, J. M., van der Goot, A. J., \& Boom, R. M. (2007). Advances in structure formation of anisotropic protein-rich foods through novel processing concepts. Trends in Food Science \& Technology, 18(11), 546-557. http://doi.org/10.1016/j.tifs.2007.05.002

Martoïa, F., Perge, C., Dumont, P. J. J., Orgéas, L., Fardin, M. A., Manneville, S., \& Belgacem, M. N. (2015). Heterogeneous flow kinematics of cellulose nanofibril suspensions under shear. Soft Matter, 11(24), 4742-4755. http://doi.org/10.1039/c5sm00530b

Mason, T. G., \& Weitz, D. A. (1995). Optical Measurements of Frequency-Dependent Linear Viscoelastic Moduli of Complex Fluids. Physical Review Letters, 74(7), 1250-1253. 
http://doi.org/10.1103/PhysRevLett.74.1250

Ovarlez, G., Mahaut, F., Deboeuf, S., Lenoir, N., Hormozi, S., \& Chateau, X. (2015). Flows of suspensions of particles in yield stress fluids. Journal of Rheology, 59(6), 1449-1486. http://doi.org/10.1122/1.4934363

Ramadugu, S. K., Chung, Y.-H., Xia, J., \& Margulis, C. J. (2009). When sugars get wet. A comprehensive study of the behavior of water on the surface of oligosaccharides. The Journal of Physical Chemistry B, 113(31), 11003-11015. http://doi.org/10.1021/jp904981v

Rathgeber, S., Beauvisage, H.-J., Chevreau, H., Willenbacher, N., \& Oelschlaeger, C. (2009). Microrheology with Fluorescence Correlation Spectroscopy. Langmuir, 25(11), 6368-6376. http://doi.org/10.1021/la804170k

Saarinen, T., Haavisto, S., Sorvari, A., Salmela, J., \& Seppälä, J. (2014). The effect of wall depletion on the rheology of microfibrillated cellulose water suspensions by optical coherence tomography. Cellulose, 21(3), 1261-1275. http://doi.org/10.1007/s10570-014-0187-5

Shemesh, N., Özarslan, E., Basser, P. J., \& Cohen, Y. (2010). Detecting diffusion-diffraction patterns in size distribution phantoms using double-pulsed field gradient NMR: Theory and experiments. The Journal of Chemical Physics, 132(3), 034703. http://doi.org/10.1063/1.3285299

Stepišnik, J., \& Callaghan, P. T. (2001). Low-frequency velocity correlation spectrum of fluid in a porous media by modulated gradient spin echo. Magnetic Resonance Imaging.

Stepišnik, J., Lahajnar, G., Zupančič, I., \& Mohoric, A. (2013). Journal of Magnetic Resonance. Journal of Magnetic Resonance, 236(C), 41-46. http://doi.org/10.1016/j.jmr.2013.08.003

Stylianopoulos, T., Diop-Frimpong, B., Munn, L. L., \& Jain, R. K. (2010). Diffusion Anisotropy in Collagen Gels and Tumors: The Effect of Fiber Network Orientation. Biophysical Journal, 99(10), 3119-3128. http://doi.org/10.1016/j.bpj.2010.08.065

Valentine, M., Kaplan, P., Thota, D., Crocker, J., Gisler, T., Prud'homme, R., et al. (2001). Investigating the microenvironments of inhomogeneous soft materials with multiple particle tracking. Physical Review E, 64(6), 061506. http://doi.org/10.1103/PhysRevE.64.061506 


\section{Summary}

In order to understand food product functionality such as elastic and flow behavior and mass transport properties, one first has to understand the multi-length-scale structure of the material. The aim of this work is to explore novel methodologies to study and characterize multi-lengthscale structures of food hydrogels under static and dynamic conditions. The focus lies on hydrogels comprising polysaccharides, because they show a rich variation in elastic and flow behavior.

The largest part of the thesis focuses on the use of nanoparticles (3-30 nm diameter) that are dissolved into the water phase of hydrogels, and whose mobility is reduced due to the presence of the polymer network. This retardation of nanoparticle self-diffusion in hydrogels relative to selfdiffusion in neat water can be used to infer structural information about the microstructure of the polymer network.

In chapter 2, an in-depth review of existing literature on this method, known as "nanoparticle diffusometry", is provided, with an emphasis on physical models of self-diffusion in polymer gels and applications in food gels. In that chapter, we distinguish between (1) nanoparticle diffusion in (heterogeneous) polymer gels and (2) nanoparticle diffusion in solutions of (semi)flexible polymers. We adhere to this categorization throughout the rest of the thesis.

In chapters 3 and 4 we first describe the design and manufacturing of tailor-made nanoparticles that are functionalized with spectroscopic labels, and the implementation of pulsed-field gradient (PFG) NMR and optical spectroscopy toolboxes for nanoparticle diffusometry. We then use these toolboxes to measure nanoparticle self-diffusion in heterogeneous $\kappa$-carrageenan (a polysaccharide) gels. These experiments reveal bimodal nanoparticle self-diffusion (i.e., there are two nanoparticle fractions with different diffusion coefficients) as previously observed in these gels by Lorén et al. The results suggest that the sub-micron structure of these gels is heterogeneous with a wide distribution of pore sizes at the sub-micron scale, leading to "sieving" of nanoparticles resulting in the observation of bimodal self-diffusion.

This hypothesis is further explored in chapter 5, where besides PFG NMR and optical spectroscopy, Overhauser dynamic nuclear polarization (ODNP)-enhanced NMR spectroscopy is employed. This method can determine the local viscosity of water surrounding the two fractions of particles. It turns out that the particle fraction with the lower apparent diffusion coefficient is in fact trapped in small, nanoscopic interstitials within the gel. The ODNP NMR experiments show that the viscosity of water surrounding the trapped particles is significantly lower than the viscosity within the larger interstitials.

Chapter 6 describes a study of nanoparticle diffusion in solutions of poly(ethylene glycol), a flexible polymer with well defined compositions and chain lengths. We use scaling laws to understand the relation between macroviscosity and "microviscosity" as apparent from the 
nanoparticle diffusivity. We show that the particles probe (near-)macroviscosity only if their size is larger than the size of the PEG polymer coils.

Another topic of this thesis is a study of the behavior of food hydrogels under dynamic conditions. To this end we use rheo-MRI velocimetry, which allows us to study the complex shear flow behavior of hydrogels that (per definition) have a yield stress. In chapter 7, we first employ nanoparticle diffusometry to study the sub-micron structure of dispersions of rigid cellulose microfibrils in the presence of carboxymethyl cellulose. Carboxymethyl cellulose is a charged cellulose derivative that succeeds to disperse the aggregation-prone cellulose microfibrils homogeneously at the sub-micron scale. Rheological characterization shows that the resulting dispersions are thixotropic yield-stress fluids. The flow properties of such fluids are well understood, but rheo-MRI experiments show that shear flow of apparently homogeneous cellulose dispersions does not resemble the flow behavior of typical thixotropic yield-stress fluids. We explain the differences by using a fluidity model to show that persistent micron-scale heterogeneity still dominates the flow behavior. 


\section{Acknowledgments}

I would like to acknowledge all who were involved in one way or the other with the work presented in this thesis. First and foremost, I would like to thank my doctoral advisors John van Duynhoven and Henk Van As for allowing me the opportunity to work at the Laboratory of Biophysics for four years, and for their dedication, enthusiasm, and originality. They have made the past four years a pleasant and memorable period.

My gratitude goes to my collaborators and co-authors who took part in the DINAMISCH project: Mieke Kleijn, Gert-Jan Goudappel, Nadia Bourouina, Freek Hoeben, Henk Janssen and Marjolein van Ruijven. The nanoparticles that this work relied upon are primarily their work. My co-authors François Mariette, Daniel Bonn, Johannes Hohlbein, Wolf Rombouts, Sandra Veen, and Krassimir Velikov are acknowledged for their critical feedback that allowed me to improve and publish my work.

Many others have contributed to this work over the past few years. I would like to thank Birgit Dekker, Kasia Grabowska, Atze Jan van der Goot, Jarno Gieteling and Ayesha Sarker for our collaboration on the determination of water distributions in soy/gluten mixtures; Frank Vergeldt and Ewoud van Velzen for their help with data analysis; Max Brouwer, Alex van der Rest and Jasper van der Gucht for their valuable contributions to the paper on diffusion in PEG solutions; Jinfeng Peng, Yuval Mulla, Tatiana Nikolaeva, Coroline Remijn, Donny Merkx, Peter Versluis, Anke Kuijk, Paul Venema, Harry Baptist, Jose Paredes, Hans Tromp, Joshua Dijksman and Marcel Workamp for helping me to come to grips with the topic of flow behavior of cellulose microfibril dispersions; Carel Feijen, Arjen Bader, Emilie Wientjes, Adrie Westphal and Gerard van Dalen for their help with the optical characterization of dye-labeled micelles; and Erich Schuster, Niklas Lorén, Songi Han, Ryan Barnes, Meike Emondts, Maria Sovago and Elena Golovina for their help with the chapter on structural and dynamical heterogeneity in polymer hydrogels.

Other coworkers helped me out with many different issues and contributed to the wonderful atmosphere in the lab. I would like to thank my roommate Alena Průšová for moral support. Gratitude goes to my Biophysics and Unilever colleagues and friends: Herbert van Amerongen, Netty Hoefakker, Adrian Voda, Shanthi Pagadala, Ruud den Adel, Patricia Heussen, Niels de Roo, Luca Bersanini, Tünde Tóth, Laura van Egmond, Pieter de Waard, Evgenia Iermak, Shazia Farouq, Edo Gerkema, Rob Koehorst, Cor Wolfs, Caner Ünlü, Folkert Hoekstra, Carlo van Mierlo, Jacques Vervoort, Fugui Xiao, Yashar Ranjbar and Lijin Tian.

Special thanks go to my family: Olga, Annelies, Hans, Jan, René and Wiep, and to Alena Průšová and Martijn Lienaerts for volunteering as my paranymphs. 



\section{Overview of completed training activities}

Discipline specific activities

Food Structure and Rheology Course

$11^{\text {th }}$ In Vivo NMR Course

International Conference on Magnetic Resonance in

Food Science

International Conference on Magnetic Resonance

Microscopy

Annual TA-COAST Programme meetings, oral

presentations

International Conference on Magnetic Resonance in

Porous Media, oral presentation

International Conference on Magnetic Resonance in

Food Science, oral presentation

CHAINS: CHemistry As INnovating Science, oral presentation

Bruker Rheo-NMR workshop, oral presentation

Dutch InterPore meeting, oral presentation

$15^{\text {th }}$ Conference of the International Association of

Colloid and Interface Science, oral presentation

Training Overhauser DNP, Songi Han laboratory at UCSB

Secondments to Unilever R\&D

\begin{tabular}{|c|c|c|}
\hline VLAG & Wageningen & 2012 \\
\hline ISMRM & Wageningen & 2012 \\
\hline AMPERE & Wageningen & 2012 \\
\hline AMPERE & Cambridge, UK & 2013 \\
\hline \multirow[t]{2}{*}{ NWO } & Lunteren, NL, & 2013- \\
\hline & Amersfoort, NL & 2015 \\
\hline AMPERE & Wellington, NZ & 2014 \\
\hline AMPERE & Cesena, IT & 2014 \\
\hline NWO & Veldhoven, NL & 2014 \\
\hline KIT & Karlsruhe, DE & 2014 \\
\hline InterPore & Vlaardingen, NL & 2014 \\
\hline \multirow[t]{2}{*}{ IACIS } & Mainz, DE & 2015 \\
\hline & $\begin{array}{l}\text { Santa Barbara, } \\
\text { CA, USA }\end{array}$ & 2016 \\
\hline
\end{tabular}

Vlaardingen, NL 20122015 


\section{General courses}

Scientific Publishing

WGS Wageningen 2013

Mobilizing your Scientific Network

WGS Wageningen 2013

Scientific Writing

WGS

Wageningen

2013

Effective Behavior in your Professional Surroundings

WGS

Wageningen

2013

Voice Matters

WGS

Wageningen

2013

Techniques for Writing and Presenting a Scientific

WGS

Wageningen

2013

Paper

Interpersonal Communication for $\mathrm{PhD}$ Students

WGS

Wageningen

2014

\section{Optionals}

Preparation of research proposal

2012

$48^{\text {th }}$ Annual Meeting of the Dutch NMR Discussion

NMRDG

Eindhoven, NL

2013

Group

$49^{\text {th }}$ Annual Meeting of the Dutch NMR Discussion

NMRDG

Oss, NL

2014

Group

$50^{\text {th }}$ Annual Meeting of the Dutch NMR Discussion

NMRDG

Utrecht, NL

2015

Group, oral presentation

Group meetings and colloquia at the Laboratory of

Wageningen

2012-

Biophysics

2016 

This research received funding from the Netherlands Organization for Scientific Research (NWO) in the framework of the Technology Area COAST. 